\title{
Photonic structures inspired by nature
}

\author{
Mathias Kolle
}

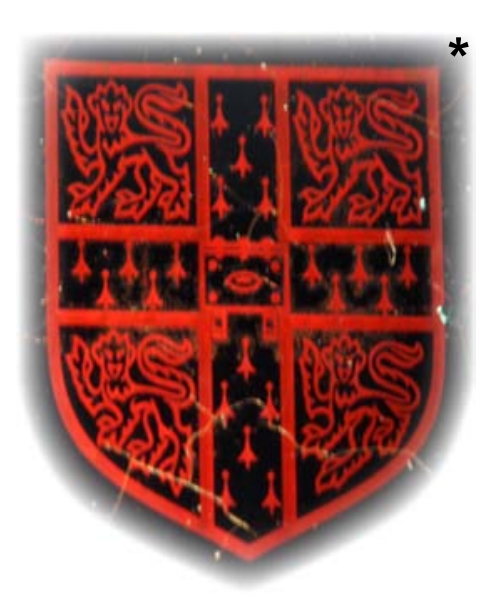

Supervisor Prof. Dr. Ullrich Steiner

A dissertation submitted for the degree of Doctor of Philosophy April 2010

\author{
University of Cambridge \\ Cavendish Laboratory \\ Cambridge Nanoscience Centre \\ Clare Hall
}

* The University of Cambridge crest, made from a nature-inspired photonic structure. 

To my family 
The vivid colours found in nature mesmerise optics experts and laymen alike. Despite the detailed scientific understanding of optics, the astonishingly varied colour palette found in nature often surpasses the optical effects that can be generated by technological means. The common characteristic feature of these so-called structural colours are intricate patterns on the $1 \mathrm{~nm}$ to $1 \mu \mathrm{m}$ length scale. The increasing understanding of natural photonic structures begets the question, how these can be mimicked by technological processes.

The scientific motivation for the replication of natural materials concerns the study of the natural systems themselves. Since the study of the natural photonic materials is often complicated by their disorder and intrinsic complexity, it is often beneficial to construct an idealised replica. Secondly, since natural structures are all unique, the variation of structural parameters afforded by structural replication facilitates the systematic study of the natural originals. There is also an increasing technological interest in complex optical materials, in particular in the field of security labelling.

Mathias Kolle's doctoral thesis describes four different aspects of natural or natureinspired optics. The principle of using replicas (rather than the natural structure itself) is demonstrated, for example, in the study of the colour response of plant petals and their role for pollinator recognition. Replica of the plant petal surfaces provided more robust samples for optical studies than the delicate petals themselves and artificially made "plant petals" were used in bee studies to explore the role of structural colour for bee recognition.

This study of a natural system is supplemented by several nature-inspired artificially made optical structures. Particularly interesting is the mimetic manufacture of the colourful wing-scales of the Papilio blumei butterfly. The important aspect here is the combination of a self-assembly process with an Angström-precision layer-deposition technique, which allows the facile manufacture of large-area structured materials with the required optical quality. This experiment also illustrates the role of fortuitous experimental design. One of the key results of this doctoral thesis, giving rise to the striking optical effect on the thesis cover, was not achieved by careful planning but by the accidental omission of one step during sample preparation. This does however, by no means lessen the scientific achievement, which in this case lies in the detailed understanding of the accidentally produced optical structure.

\section{Ullrich Steiner}

01 July 2010 


\section{Contents}

Introduction $\quad 1$

1 Theoretical aspects of photonic structures $\quad 7$

1.1 Reflection and refraction at optical interfaces . . . . . . . . . . . 8

1.2 Thin film interference . . . . . . . . . . . . . . . . . 10

1.3 Multilayer reflectivity . . . . . . . . . . . . . . . 12

1.3.1 Qualitative analysis of multilayer interference . . . . . . . . 12

1.3.2 Quantitative analysis of multilayer interference . . . . . . . . 14

1.4 Band-gaps of one-dimensional photonic crystals . . . . . . . . . . . 17

1.5 Multilayer resonance cavities . . . . . . . . . . . . . 25

1.6 Diffraction from periodic surface structures . . . . . . . . . . . . 26

1.6.1 Diffraction in the Fraunhofer approximation . . . . . . . . . 27

1.6.2 Finite-Difference Time-Domain modelling of diffraction . . . . . 29

$\begin{array}{lll}2 & \text { Structural colours in nature } & 37\end{array}$

2.1 Structural colours in the animal kingdom . . . . . . . . . . . . . 38

2.1.1 Simple multilayers - the Japanese jewel beetle . . . . . . . . 38

2.1.2 Complex multilayer arrangements in butterfly and moth structures 40

2.2 Floral diffractive structures . . . . . . . . . . . . . . . 48

2.2.1 Diffraction and iridescence from striations on flower petals . . . 49

2.2.2 Near-UV reflectivity enhancement by grating-like striations . . . 60

2.3 Conclusion . . . . . . . . . . . . . . . . . 65

3 Materials and techniques $\quad 75$

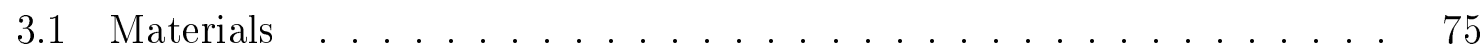

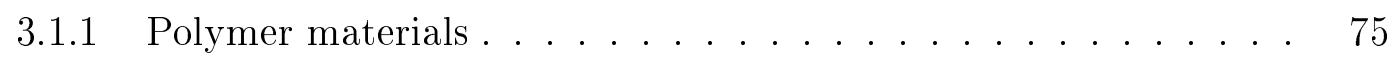

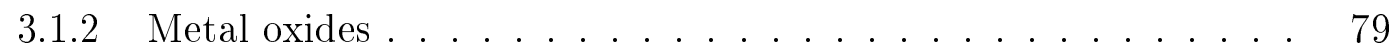

3.2 Techniques - multilayer production . . . . . . . . . . . 80

3.2.1 Sequential spin-coating . . . . . . . . . . . . 80

3.2 .2 Floating and stacking . . . . . . . . . . . . . . . . . 81

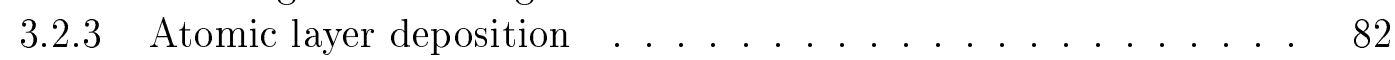

3.3 Techniques - pattern creation and transfer . . . . . . . . . . 86

3.3.1 Colloid templating . . . . . . . . . . . . 86

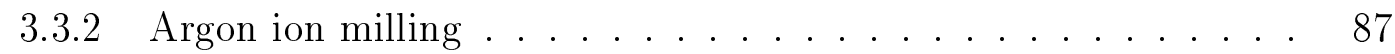

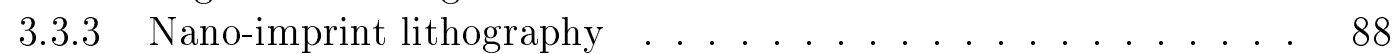

3.3.4 Dental wax casting ............................. 90

3.4 Techniques - sample characterisation . . . . . . . . . . . . . . 91

3.4 Gonio-spectroscopy . . . . . . . . . . . . . . . . 91

3.4 .2 Micro-spectroscopy ........................ 93 


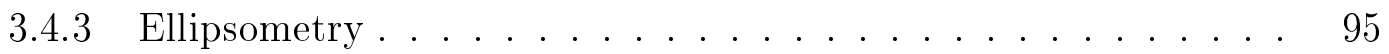

4 Static and tuneable one-dimensional photonic structures $\quad 107$

4.1 Static one-dimensional optical devices . . . . . . . . . . . . . . . . . 107

4.1.1 Organic Bragg reflectors . . . . . . . . . . . . . . . . . 108

4.1.2 Organic resonance cavities . . . . . . . . . . . . . 113

4.2 Stretch-tuneable dielectric mirrors and optical microcavities . . . . . . 117

4.2.1 Sample preparation and experimental setup . . . . . . . . . 118

4.2.2 Cavity design and modelling . . . . . . . . . . . . . . . . . 122

4.2.3 Results and discussions . . . . . . . . . . . . . . . . . . . . 123

4.3 Conclusion . . . . . . . . . . . . . . . . . . . 128

5 Microfabrication of photonic structures with higher dimensionality 135

5.1 Template-assisted ion milling . . . . . . . . . . 136

5.1 .1 The ion milling template . . . . . . . . . . . 136

5.1 .2 Micro-cones . . . . . . . . . . . . . . . 137

5.1 .3 Gold crowns on micro-cones . . . . . . . . . . . . . . 140

5.1 .4 Multilayer patterning by ion milling . . . . . . . . . . . . . 142

5.2 Nano-imprinting of photonic structures . . . . . . . . . . . . . 149

5.2.1 Imprinting with micro-cone masters . . . . . . . . . . . . . . 149

5.2.2 Replication of micro-cones for flexible cell substrates . . . . . 151

5.2.3 Fabrication of an imprint master for the replication of butterfly wing scale patterns . . . . . . . . . . . . . . . 152

5.2 .4 Multilayer patterning by nano-imprinting . . . . . . . . . . . 154

5.3 Atomic layer deposition on structured substrates . . . . . . . . . . . 156

5.4 Conclusion . . . . . . . . . . . . . . . . . 158

6 Mimicry of Papilio blumei's colourful wing scale structure $\quad 165$

6.1 The role model . . . . . . . . . . . . . . . 166

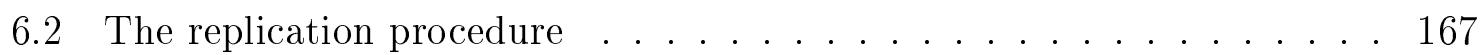

6.3 A structural replica . . . . . . . . . . . . . . . . 169

6.4 A structurally modified replica with enhanced optical performance . . . 174

6.5 Conclusion . . . . . . . . . . . . . . . . . . 179

$\begin{array}{ll}\text { Conclusions and future work } & 183\end{array}$

$\begin{array}{lr}\text { Acknowledgements } & 189\end{array}$

$\begin{array}{ll}\text { Related publications } & 191\end{array}$ 


\section{Introduction}

Light and our ability to detect light in the wavelength range of $\sim 400-700 \mathrm{~nm}$ enable us to identify objects by their shape, size and colour, thereby providing us with vital information about our surroundings ${ }^{1}$.

Objects that stand in strong contrast to the environment due to their distinct colour or brightness catch our eye. Most of the colours around us originate from selective absorption caused by pigmentation incorporated in the surface or body of an object. However, particularly intense and bright colours result from the interaction of light with micro- and nano-structures, which cause colour by interference, coherent scattering or diffraction without any absorption. These colours are termed "structural colours" and structures that, by modulation of light, cause structural colours are part of the family of "photonic" structures. Photonic structures are regular structures with periodicities on the order of the wavelength of light.

Structural colours have fascinated researchers for centuries and early successes in gaining understanding of the role of micro- and nano-structures are credited to Hooke (1665) [2], Newton (1704) [3] and Lord Rayleigh (1917) [4]. First imaging and a detailed study of structural elements that induce structural colours commenced with Anderson and Richards in 1942 [5], following the introduction of the electron microscope.

In recent years, the study of natural structural colours has found increasing interest due to rapid progress in scanning / transmission electron microscopy and optical spectroscopy. These techniques allow scientists to investigate in great detail the complex micro- and nano-structures with unique optical characteristics, that have evolved in nature for millions of years [6-10]. The first part of this thesis is focussed on the the-

\footnotetext{
${ }^{1}$ If you really want to get a glimpse of the importance of your sense of vision, you should go to a blind-dining restaurant and enjoy the surprising helplessness that overwhelms you when you try to eat and identify food and wine purely by taste in a pitch-black environment where your sense of vision is completely useless. Not easy at all. It is truly a refreshing, fantastic experience [1].
} 
oretical concepts needed to explain natural structural colours and the identification of natural photonic structures reported in the literature. The most versatile photonic systems found in nature often contain multilayered substructures and diffraction-grating like elements. Consequently our focus lies on the theoretical tools for treating these systems (Chapter 1) and natural model structures that incorporate periodically deformed multilayer arrangements (Chapter 2).

Structural colours have been extensively studied in the animal kingdom however, only very few plants have been shown to exploit photonic structures. In this work we have explored the optical properties of floral structural colours caused by micro-striations found on the petals of wild flowering plant species (second part in Chapter 2). We studied the morphology and optical signature of grating-like flower petal structures which were found to induce light diffraction. In collaboration with biologists the results were used to show that pollinators can detect iridescence resulting from the interaction of light with periodic micro- and nano-structures [11]. This suggests, that flowers might exploit micro-structural elements to manipulate their optical appearance to increase their attraction to pollinators, which has not previously been considered.

For many reoccurring optical problems, including the realisation of high reflectivity or transmission, strong light polarisation, spectral filtering, iridescence or dichroism, nature provides multiple answers in the form of micro- and nano-structures of great morphological variety. These have developed independently in unrelated organisms to serve similar purposes. Nature thus offers an enormous number of blue-prints for multifunctional micro- and nanostructures that show outstanding, distinctive and dynamic colouration. This abundance of solutions invites scientists, who do not have a million years for evolutionary research and development, to play copy-cat [12]. We can study nature and gain invaluable knowledge for the creation of novel photonic materials for "moulding the flow of light" [13] that may form the basis of future optical devices for applications in medical diagnostics, communication, high-speed data transfer, information processing and precision manufacturing with functionalities that go beyond the capacities of current state-of-the-art equipment. For this reason biomimetic approaches for the development of novel photonic structures have attracted increasing 
research interest and also play a major role in this work. In this context we explore unique material combinations for potential use in well established optical elements such as Bragg filters and mirrors or optically pumped light emitting diodes (Chapter 3 and Chapter 4). We demonstrate stretch-tuneable Bragg mirrors and resonance cavities using novel scaleable assembly techniques and study the optical performance and tunability of these stretchable multilayer structures (Chapter 4) [14]. We investigate nano-fabrication techniques, suitable for large scale time-efficient indus- trial fabrication of complexly shaped photonic structures Chapter 3 and Chapter 5). We focus on nano-imprinting, template-assisted ion-milling and atomic layer deposition. The fabrication and optical investigation of model structures with morphologies, that cannot easily be achieved with industrial semiconductor technology are presented.

Finally, we demonstrate the replication of the photonic structure found on the wing scales of the Indonesian swallowtail butterfly Papilio blumei (Chapter 6) [15]. Although attempts have been made by other research groups, the 5 - $10 \mu \mathrm{m}$ wide concavities cladded with a cuticle-air multilayer have not previously been mimicked satisfyingly with large areal coverage. We have successfully replicated the intricate photonic structure on the $\mathrm{cm}^{2}$-scale by combining colloidal templating, electro-deposition and atomic layer deposition. Further insight into the propagation of light within concave multilayer structures was achieved by a detailed study of their optical performance. We also show that a small conceptual modification of the original photonic structure leads to a completely different optical effect. Any freely chosen colour and its complementary hue can be separated and reflected into different directions, accompanied by a particular polarisation conversion. Since the procedures are up-scaleable, these biomimetic photonic structures could be suitable for industrial applications in security printing, encoding of information, non-emissive display technology and other fields where distinct colours play a role. 



\section{References}

[1] "Dans le Noir". 30-31 Clerkenwell Green EC1R 0DU - London, UK (opened in spring 2006).

[2] Hooke, R. Micrographia (reprinted by Dover Publications, 2003).

[3] Newton, I. Opticks (reprinted by Dover Publications, 1952).

[4] Lord Rayleigh. On the reflection of light from a regularly stratified medium. Proc. Roy. Soc. A 39, 565-577 (1917).

[5] Anderson, T. F. \& Richards, A. G. An electron microscope study of some structural colours of insects. J. Appl. Phys. 13, $748 Đ 758$ (1942).

[6] Vukusic, P. \& Sambles, J. R. Photonic structures in biology. Nature 424, 852-855 (2003).

[7] Vukusic, P. Advanced photonic systems on the wing-scales of Lepidoptera. In Gorb, S. N. (ed.) Functional surfaces in biology: Little structures with big effects (Springer, 2009).

[8] Parker, A. 515 million years of structural colour. J. Opt. A - Pure Appl. Op. 2, R15-R28 (2000).

[9] Parker, A. \& Martini, N. Structural colour in animals - simple to complex optics. Opt. Laser Technol. 38, 315-322 (2006).

[10] Kinoshita, S. \& Yoshioka, S. Structural colors in nature: The role of regularity and irregularity in the structure. ChemPhysChem 6, 1442-1459 (2005).

[11] Whitney, H., Kolle, M., Andrew, P., Chittka, L., Steiner, U. \& Glover, B. J. Floral iridescence, produced by diffractive optics, acts as a cue for animal pollinators. Science 323, 130-133 (2009). 
[12] Parker, A. \& Townley, H. Biomimetics of photonic nanostructures. Nature Nanotech. 2, 347-353 (2007).

[13] Joannopoulos, J. D., Johnson, S. G., Winn, J. N. \& Meade, R. D. Photonic crystals: molding the flow of light (Princeton University Press, 2008).

[14] Kolle, M., Zheng, B., Gibbons, N., Baumberg, J. J. \& Steiner, U. Stretch-tuneable dielectric mirrors and optical microcavities. Opt. Express 18, 4356-4364 (2010).

[15] Kolle, M., Salgard-Cunha, P. M., Scherer, M. R. J., Huang, F., Mahajan, S., Vukusic, P., Baumberg, J. J., \& Steiner, U. Mimicry of Papilio blumei's colourful wing scale structure. Nature Nanotech. (2010). 


\section{Chapter 1}

\section{Theoretical aspects of photonic structures}

The fabrication of novel photonic devices is usually preceded by a design phase, involving the theoretical modelling of the device's optical behaviour, based on the properties of the applied materials. Various techniques have been developed for the theoretical study of light scattering, interference, or diffraction caused by photonic micro- and nano-structures [1-4]. Analytical treatments are only possible for some particular cases and many approaches have to rely on approximations or simulation of the electromagnetic fields in the structure of interest. Common approaches include:

- Transfer matrix techniques [2],

- Finite-Difference Time-Domain (FDTD) and Finite-Difference Frequency-Domain (FDFD) simulations [3],

- Photonic band-gap calculations based on plane wave expansion [4].

These theoretical methods are very powerful tools, if one keeps aware of their limitations and interprets results correctly.

One of the main aspects of this thesis is the study of periodic multilayer structures, also called one-dimensional photonic crystals. Interference effects, occurring in flat multilayer structures can be treated analytically by using transfer matrix techniques [2] or by extending the derivation of the reflection coefficients of a single thin film to the case of any number of layers [5]. These calculations give precise solutions but cannot easily be extended to the treatment of non-planar structures.

The interaction of light with two- or three-dimensional photonic crystals can be investigated computationally with the freely available "MIT Photonic-Bands" package 
(MPB) [6] or the "MIT Electromagnetic Equation Propagation" software (MEEP) [7]. MPB computes definite-frequency eigenstates (harmonic modes) of Maxwell's equations in periodic dielectric structures for arbitrary wave-vectors in order to determine dispersion diagrams and electromagnetic modes in the dielectric medium. MEEP is a Finite-Difference Time-Domain (FDTD) simulation package for the computation of transmission / reflection spectra, field patterns, resonant modes and frequencies in dielectric structures.

In this chapter the basics of reflection, refraction and interference in thin films are laid out and applied to the case of multilayer stacks made from lossless, linear dielectric materials. Several methods used for the characterisation of one-dimensional photonic crystals and simple diffraction elements are investigated and finally the suitability of MEEP for the modelling of diffraction from arbitrarily shaped periodic micro-structures is discussed.

\subsection{Reflection and refraction at optical interfaces}

Assume a light wave impinging on an interface $n_{1} \mid n_{2}$ between two, optically smooth dielectric materials with distinct refractive indices $n_{1}$ and $n_{2}$, at an angle $\theta_{1}$ with respect to the interface normal. Part of the wave is specularly reflected, while the rest is refracted (Fig. 1.1 a). The reflected light leaves the interface obeying the condition $\theta_{\mathrm{r}}=-\theta_{1}$, where $\theta_{\mathrm{r}}$ is the angle between the propagation direction of the reflected light and the interface normal. The refracted light propagates into the second medium at an angle $\theta_{2}$ given by Snell's law

$$
n_{1} \cdot \sin \theta_{1}=n_{2} \cdot \sin \theta_{2}
$$

The electric field amplitudes $E_{\mathrm{r}}$ and $E_{\mathrm{t}}$ and intensities $R$ and $T$ of the reflected and the transmitted light depend on the polarisation of the incident light. Light that is polarized in the plane of incidence is referred to as parallel polarized light with field amplitude $E_{\|}$. Light, that is polarized in the plane of the optical interface (normal to 

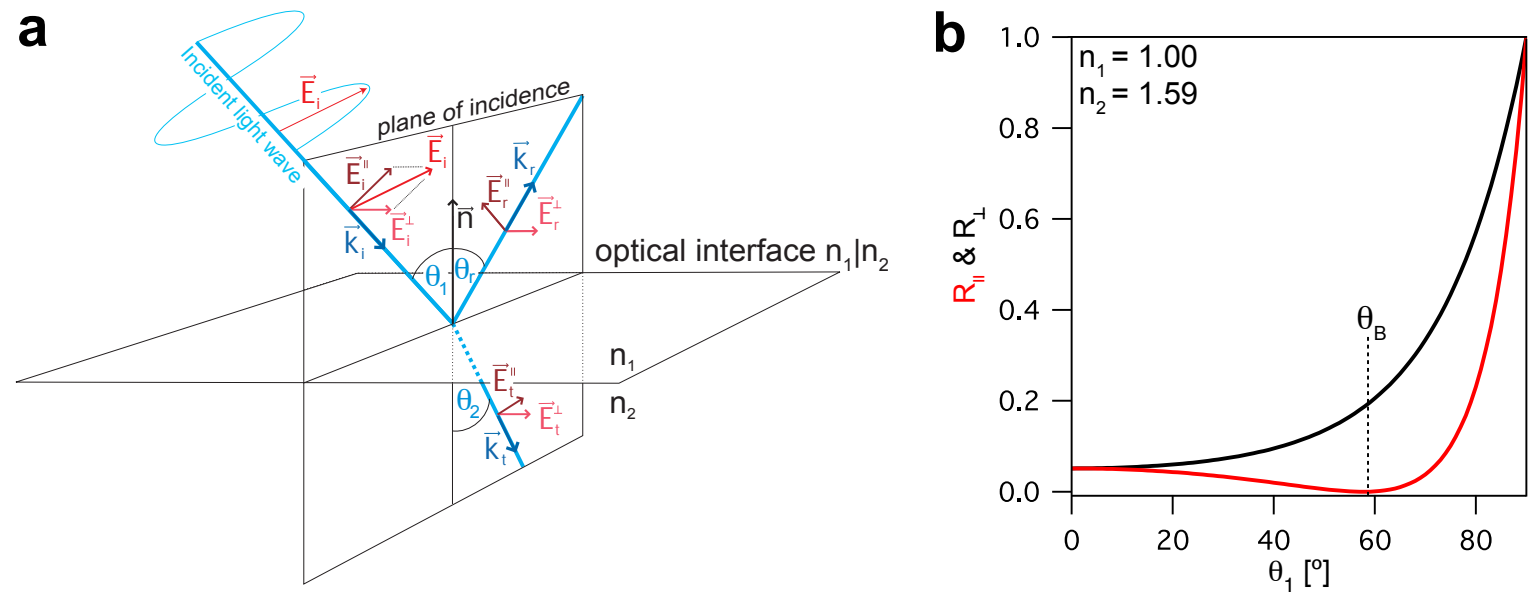

Figure 1.1: Reflection and refraction at an optical interface $n_{1} \mid n_{2}$. a) The wave vectors of the incident, transmitted and reflected waves are $\vec{k}_{\mathrm{i}}, \vec{k}_{\mathrm{t}}$ and $\vec{k}_{\mathrm{r}}$. The electric fields are $\vec{E}_{\mathrm{i}}, \vec{E}_{\mathrm{t}}$ and $\vec{E}_{\mathrm{r}}$, decomposed into their components parallel and perpendicular to the plane of incidence, $\vec{E}^{\|}$and $\vec{E}^{\perp}$. b) Reflectivity of an air $\mid$ polystyrene interface for parallel and perpendicularly polarised light, $R_{\|}$and $R_{\perp}$, as a function of incidence angle $\theta_{1}$. At the Brewster angle $\theta_{\mathrm{B}}=57.8^{\circ}, R_{\|}$vanishes and the reflected light is completely polarised perpendicularly to the plane of incidence.

the plane of incidence) is termed perpendicularly polarized light with field amplitude $E_{\perp}$. Fresnel's reflection and transmission coefficients

$$
\begin{aligned}
& r_{\|}=\frac{E_{\mathrm{r}, \|}}{E_{\mathrm{i}, \|}}=\frac{n_{2} \cos \theta_{1}-n_{1} \cos \theta_{2}}{n_{2} \cos \theta_{1}+n_{1} \cos \theta_{2}}=\frac{\tan \left(\theta_{1}-\theta_{2}\right)}{\tan \left(\theta_{1}+\theta_{2}\right)} \\
& r_{\perp}=\frac{E_{\mathrm{r}, \perp}}{E_{\mathrm{i}, \perp}}=\frac{n_{1} \cos \theta_{1}-n_{2} \cos \theta_{2}}{n_{1} \cos \theta_{1}+n_{2} \cos \theta_{2}}=-\frac{\sin \left(\theta_{1}-\theta_{2}\right)}{\sin \left(\theta_{1}+\theta_{2}\right)} \\
& t_{\|}=\frac{E_{\mathrm{t}, \|}}{E_{\mathrm{i}, \|}}=\frac{2 n_{1} \cos \theta_{1}}{n_{2} \cos \theta_{1}+n_{1} \cos \theta_{2}} \\
& t_{\perp}=\frac{E_{\mathrm{t}, \perp}}{E_{\mathrm{i}, \perp}}=\frac{2 n_{1} \cos \theta_{1}}{n_{1} \cos \theta_{1}+n_{2} \cos \theta_{2}}
\end{aligned}
$$

provide a measure for the amplitudes of reflected and transmitted light.

The intensities of parallel- and perpendicularly-polarised, reflected and transmitted light $R_{\|}, R_{\perp}$ and $T_{\|}, T_{\perp}$ are then given by

$$
\begin{aligned}
R_{\|, \perp} & =r_{\|, \perp}^{2} \\
T_{\|, \perp} & =\frac{n_{2}}{n_{1}} \cdot t_{\|, \perp}^{2} .
\end{aligned}
$$


For linearly polarized, incident light with a polarization angle $\psi$ with respect to the plane of incidence the reflectivity is

$$
R=R_{\|} \cdot \cos ^{2} \psi+R_{\perp} \cdot \sin ^{2} \psi
$$

It is worth noting, that $R_{\perp}$ increases monotonously with increasing angle of incidence, while $R_{\|}=0$ at the Brewster angle (Fig. $1.1 \mathrm{~b}$ ). From the first line in Equation 1.2, the Brewster angle, $\theta_{1}=\theta_{\mathrm{B}}$, is deduced from

$$
\tan \left(\theta_{2}+\theta_{\mathrm{B}}\right)=0
$$

that is (using Snell's law)

$$
\theta_{\mathrm{B}}=\arctan \left(\frac{n_{2}}{n_{1}}\right)
$$

\subsection{Thin film interference}

The reflectivity of a thin, non absorbing layer of thickness $d_{1}$ with refractive index $n_{1}$, bound by two semi-infinite media with refractive indices $n_{0}$ and $n_{2}$, can be determined theoretically by summation over the amplitudes of all the light beams which leave the layer in reflection. Those beams might have incurred multiple reflections within the layer (Fig 1.2). In the following, the Fresnel reflection and transmission coefficients of each of the two interfaces in the system are denoted $r_{i j}$ or $t_{i j}$, with the index $i$ denoting the medium from which a light beam hits an optical interface and index $j$ representing the medium on the other side of that interface. The polarisation of the beam has to be taken into account in the determination of $r_{i j}$ and $t_{i j}$ but the subsequent treatment is identical for both polarisations, allowing us to drop the indices $\perp$ and $\|$. From Fresnel's formulae it follows that $r_{i j}=-r_{j i}$. The length of the optical path travelled by a light ray in the thin layer dictates the shift in phase

$$
\delta_{m}=(m+1) \cdot \delta=(m+1) \cdot \frac{2 \pi}{\lambda} n_{1} d_{1} \cos \theta_{1},
$$




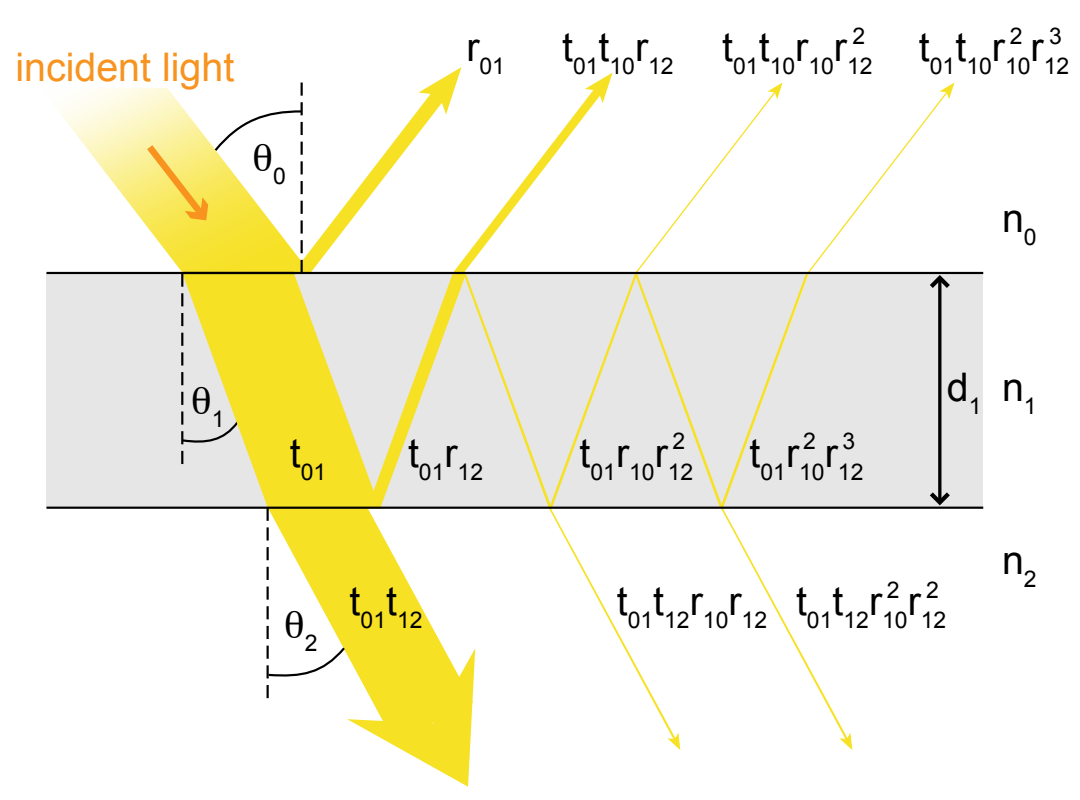

Figure 1.2: Thin film reflectivity. Many of the light beams that are contributing to the overall reflectivity of the thin film have incurred multiple reflections in the layer, which determine their final amplitude when they leave the layer, based on Fresnel's reflection and transmission coefficients.

with $\theta_{1}$ the angle of refraction in the layer and $m$ the number of internal reflections. All the rays contributing to the overall reflectivity $R$ of the film, have incurred an odd number of internal reflections (that is $m+1=2 n$, where $n$ is a natural number). By summation over the amplitudes of these beams, taking into account their phase shift, the complex reflected amplitude $A_{r}$ amounts to

$$
\begin{aligned}
A_{r} & =r_{01}+t_{01} t_{10} r_{12} \mathrm{e}^{-2 \mathrm{i} \delta} \sum_{n=0}^{\infty}(-1)^{n}\left(r_{01} r_{12}\right)^{n} \mathrm{e}^{-2 \mathrm{i} n \delta} \\
& =r_{01}+\frac{t_{01} t_{10} r_{12} \mathrm{e}^{-2 \mathrm{i} \delta}}{1+r_{01} r_{12} \mathrm{e}^{-2 \mathrm{i} \delta}} \\
& =\frac{r_{01}+r_{12} \mathrm{e}^{-2 \mathrm{i} \delta}}{1+r_{01} r_{12} \mathrm{e}^{-2 \mathrm{i} \delta}} .
\end{aligned}
$$

For the last equality in Equation 1.6 the relation

$$
t_{01} t_{10}=\sqrt{1-r_{01}^{2}} \sqrt{1-r_{10}^{2}}=1-r_{01}^{2}
$$


has been used, essentially signifying the conservation of energy upon reflection and refraction. Finally the reflectivity of a thin layer is given by

$$
R=A_{r} A_{r}^{*}=\frac{r_{01}^{2}+2 r_{01} r_{12} \cos 2 \delta+r_{12}^{2}}{1+2 r_{01} r_{12} \cos 2 \delta+r_{01}^{2} r_{12}^{2}}
$$

\subsection{Multilayer reflectivity}

The reflectance behaviour of stacks of transparent, dielectric layers with distinct refractive indices results from the interference of light reflected from the different optical interfaces in the stack. This section focuses on multilayer stacks with $N$ alternating layers of two different materials with refractive index $n_{1}>n_{2}$ and film thicknesses $d_{1}$, $d_{2}$. The stack is enclosed at the top and bottom by two (not necessarily different) media with refractive indices $n_{0}$ and $n_{\mathrm{s}}$. Coming from the upper medium, light impinges on the multilayer stack at an angle $\theta_{0}$ (Fig 1.3).

\subsubsection{Qualitative analysis of multilayer interference}

Simple relations for the determination of the peak reflectance wavelength can be deduced by specifying the conditions for constructive interference of light beams that are reflected from the different optical interfaces in the stack (Fig 1.3). Constructive interference of light, that is reflected off two consecutive interfaces $n_{1} \mid n_{2}$, is given, if the optical path length difference of the two light rays is equal to $m \cdot \lambda$, where $m$ is a positive number. Purely geometrical considerations, taking into account Snell's law, lead to the relation

$$
m \cdot \lambda=2\left(d_{1} \cdot \sqrt{n_{1}^{2}-n_{0}^{2} \sin ^{2} \theta_{0}}+d_{2} \cdot \sqrt{n_{2}^{2}-n_{0}^{2} \sin ^{2} \theta_{0}}\right)
$$

which determines the wavelength $\lambda$ at which constructive interference occurs.

For the interference of two rays that are reflected off two neighbouring interfaces $n_{1}\left|n_{2}-n_{2}\right| n_{1}$ (or $n_{2}\left|n_{1}-n_{1}\right| n_{2}$ ), an additional phase change of $\pi$ has to be taken into 


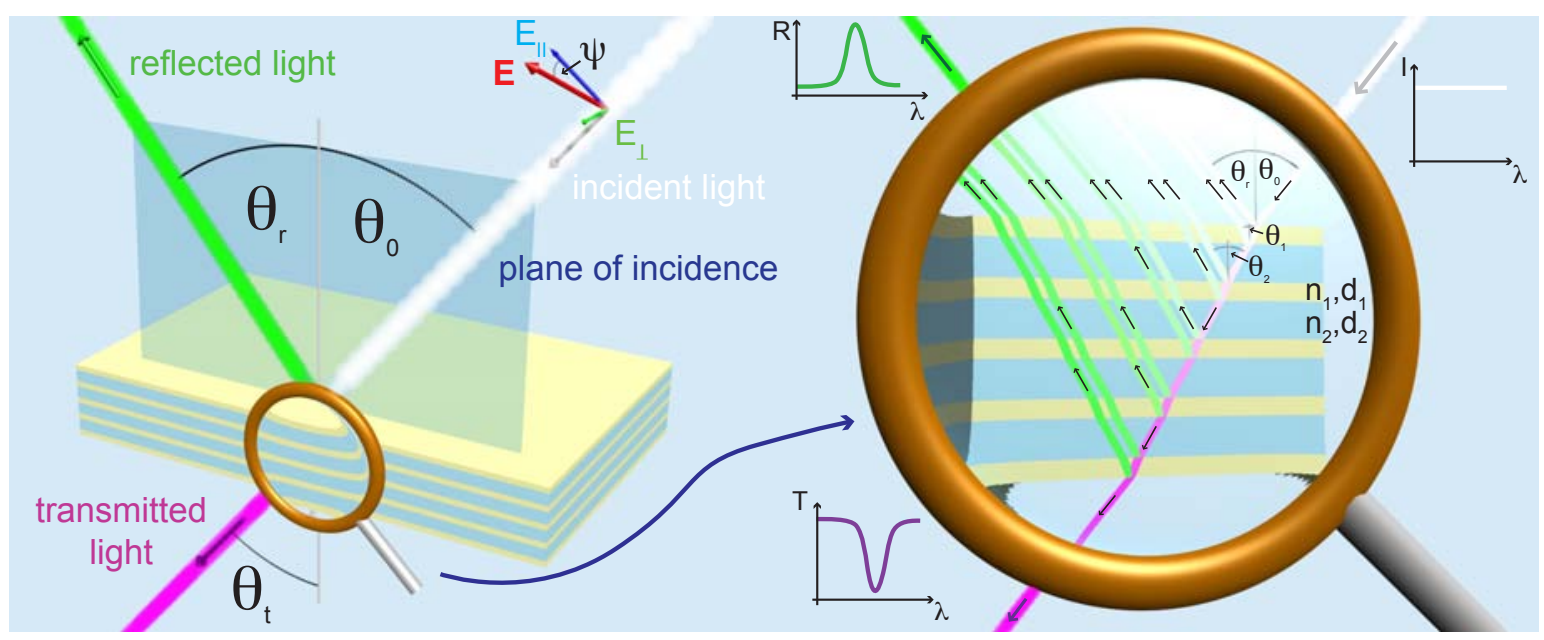

Figure 1.3: Multilayer interference. Reflection and transmission of light by a multilayer. Light beams reflected at the different interfaces in the multilayer stack interfere and cause the characteristic reflection and transmission spectra depending on the thickness and refractive index of the constituent materials.

account. This is justified by the fact that for all angles of incidence the Fresnel coefficients $r_{\|}, r_{\perp}$ of the neighbouring interfaces are related by

$$
r_{\|, \perp}^{n_{1} \mid n_{2}}=-r_{\|, \perp}^{n_{2} \mid n_{1}}
$$

resulting in a additional contribution of $\pi$ to the phase difference between two light beams reflected from the neighbouring interfaces. Consequently, constructive interference occurs, if the optical path difference is equal to $\left(m^{\prime}+\frac{1}{2}\right) \cdot \lambda$, where $m^{\prime}$ is a positive number. This leads to

$$
\left(m^{\prime}+\frac{1}{2}\right) \cdot \lambda=2 d_{j} \cdot \sqrt{n_{j}^{2}-n_{0}^{2} \sin ^{2} \theta_{0}}
$$

where $j$ stands for the respective layer.

Multilayer interference structures that satisfy Equations 1.8 and 1.9 are called "ideal" multilayers [8]. For non-ideal multilayer stacks the peak reflectance wavelength is determined by Equation 1.8.

At normal incidence of light and $m^{\prime}=0$, Equation 1.9 simplifies to $\frac{\lambda}{4}=n_{j} d_{j}$, hence the common name quarter-wave stack for ideal multilayer arrangements. 


\subsubsection{Quantitative analysis of multilayer interference}

\section{Rouard's technique}

The reflectivity of a multilayer structure can be calculated as a function of the relevant parameters wavelength of the incident light $\lambda$, refractive indices $n_{1}, n_{2}$, and film thicknesses $d_{1}, d_{2}$ of the individual layers, layer number $N$, and angle of light incidence $\theta_{0}$ using Rouard's technique [5]. Assuming the dielectric materials in the multilayer are transparent, the $i^{\text {th }}$ layer is fully characterised by its real-valued refractive index $n_{i}$ and its layer thickness $d_{i}$. The bottom $N-(i-1)$ layers of the stack are referred to as the $i^{\text {th }}$ sub-stack. The $i^{\text {th }}$ layer in the entire multilayer is the first layer of the $i^{\text {th }}$ sub-stack. Consequently, the $1^{\text {st }}$ sub-stack is the entire multilayer itself, while the $(N+1)^{\text {th }}$ sub-stack is the bare substrate. Starting at the substrate, the complex effective reflection coefficient $\tilde{r}_{i}=\rho_{i} \mathrm{e}^{\mathrm{i} \Delta_{i}}$ of the $i^{\text {th }}$ sub-stack is calculated by iteration. Insertion of the effective reflection coefficient $\tilde{r}_{i+1}$ of the $(i+1)^{\text {th }}$ sub-stack, that underlies the $i^{\text {th }}$ layer, in Equation 1.6 gives

$$
\tilde{r}_{i}=\rho_{i} \mathrm{e}^{\mathrm{i} \Delta_{i}}=\frac{r_{i-1, i}+\rho_{i+1} \mathrm{e}^{\mathrm{i} \Delta_{i+1}} \mathrm{e}^{-2 \mathrm{i} \delta_{i}}}{1+r_{i-1, i} \rho_{i+1} \mathrm{e}^{\mathrm{i} \Delta_{i+1}} \mathrm{e}^{-2 \mathrm{i} \delta_{i}}},
$$

where $r_{i-1, i}$ is the reflection coefficient of the optical interface $n_{i-1} \mid n_{i}$ between $(i-1)^{\text {th }}$ and $i^{\text {th }}$ layer and $\delta_{i}=\frac{2 \pi}{\lambda} n_{i} d_{i} \cos \theta_{0}$ is the phase change that the light wave picks up as it traverses the $i^{\text {th }}$ layer.

\section{Results}

Rouard's technique was implemented in a MatLab algorithm. For user-friendliness, the program is equipped with a graphical user interface which allows for in-situ variation of the multilayer parameters, such as refractive indices, film thicknesses, layer number, substrate and super-strate refractive indices or light incidence angle. Furthermore, a transfer matrix algorithm [2] has been compiled in a $\mathrm{C}++$ routine to verify the data, acquired with the implementation of Rouard's method. Both programs produce matching results. Optical absorption in the individual layers or in the substrate material can 

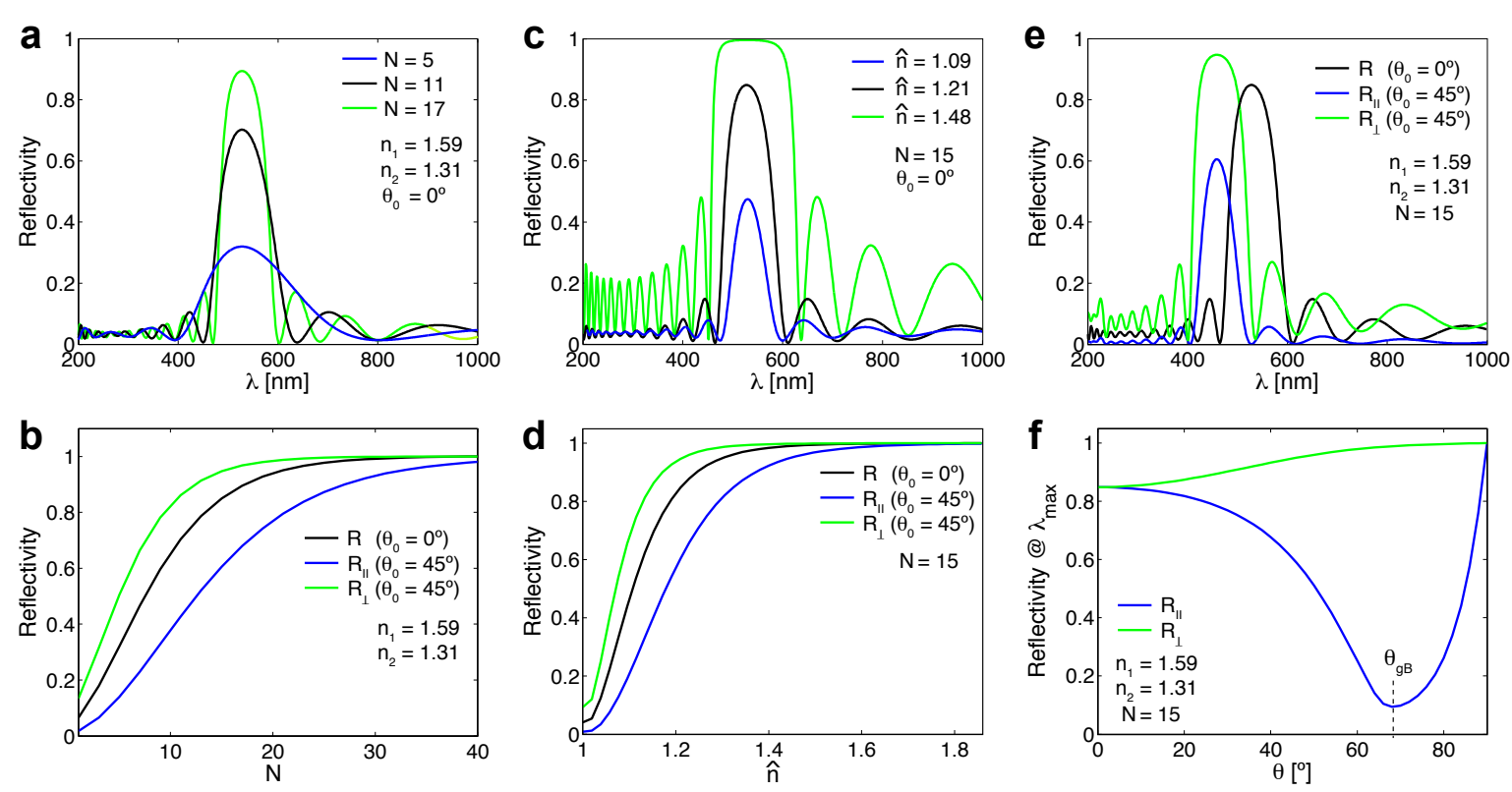

Figure 1.4: Multilayer reflectivity. a) Reflectivity versus wavelength $\lambda$ for different layer numbers $N$ at normal light incidence $\theta_{0}=0^{\circ}$. b) Reflectivity of parallel- and perpendicularly-polarised light versus layer number $N$ for $\theta_{0}=0^{\circ}$ and $\theta_{0}=45^{\circ}$. c) $R(\lambda)$ for different refractive index contrasts $\hat{n}=\frac{n_{1}}{n_{2}}$ at constant layer number $N=15$ and $\theta_{0}=0^{\circ}$. d) $\mathrm{R}_{\|}$and $\mathrm{R}_{\perp}$ versus $\hat{n}$ for $\theta_{0}=0^{\circ}$ and $\theta_{0}=45^{\circ}, \mathrm{N}=15$. e) $R_{\|}$and $R_{\perp}$ versus wavelength for $\theta_{0}=0^{\circ}$ and $\theta_{0}=45^{\circ}, N=15, \hat{n}=1.21$. f) $R_{\|}\left(\lambda_{\max }\right)$ and $R_{\perp}\left(\lambda_{\max }\right)$ versus $\theta_{0}, N=15, \hat{n}=1.21$.

be accounted for by working with complex refractive indices.

The reflectivity of a multilayer structure composed of two different materials with refractive indices $n_{1}, n_{2}$ depends on the number of layers $N$, the refractive index contrast $\hat{n}=\frac{n_{1}}{n_{2}}$ (assuming $n_{1}>n_{2}$ ), the polarisation of the incident light $\psi$, the thickness of the individual layers $d_{1}, d_{2}$ and the angle of light incidence $\theta_{0}$. The influence of these parameters on the reflectivity of ideal multilayer quarter-wave stacks is investigated below. The reflectivity also depends on the underlying substrate and the super-strate but this dependence is not discussed in detail here.

With increasing number of layers $N$ the reflectivity increases while the bandwidth of the reflection peak decreases (Fig. $1.4 \mathrm{a}$ ). At light incidence angles $\theta_{0} \neq 0^{\circ}$, the increase in reflectivity is more pronounced for perpendicularly-polarised light (Fig. 1.4 b). For small enough layer numbers there is a significant difference between $R_{\perp}$ and $R_{\|}$and consequently unpolarised light becomes partly polarized upon reflection from a multi- 

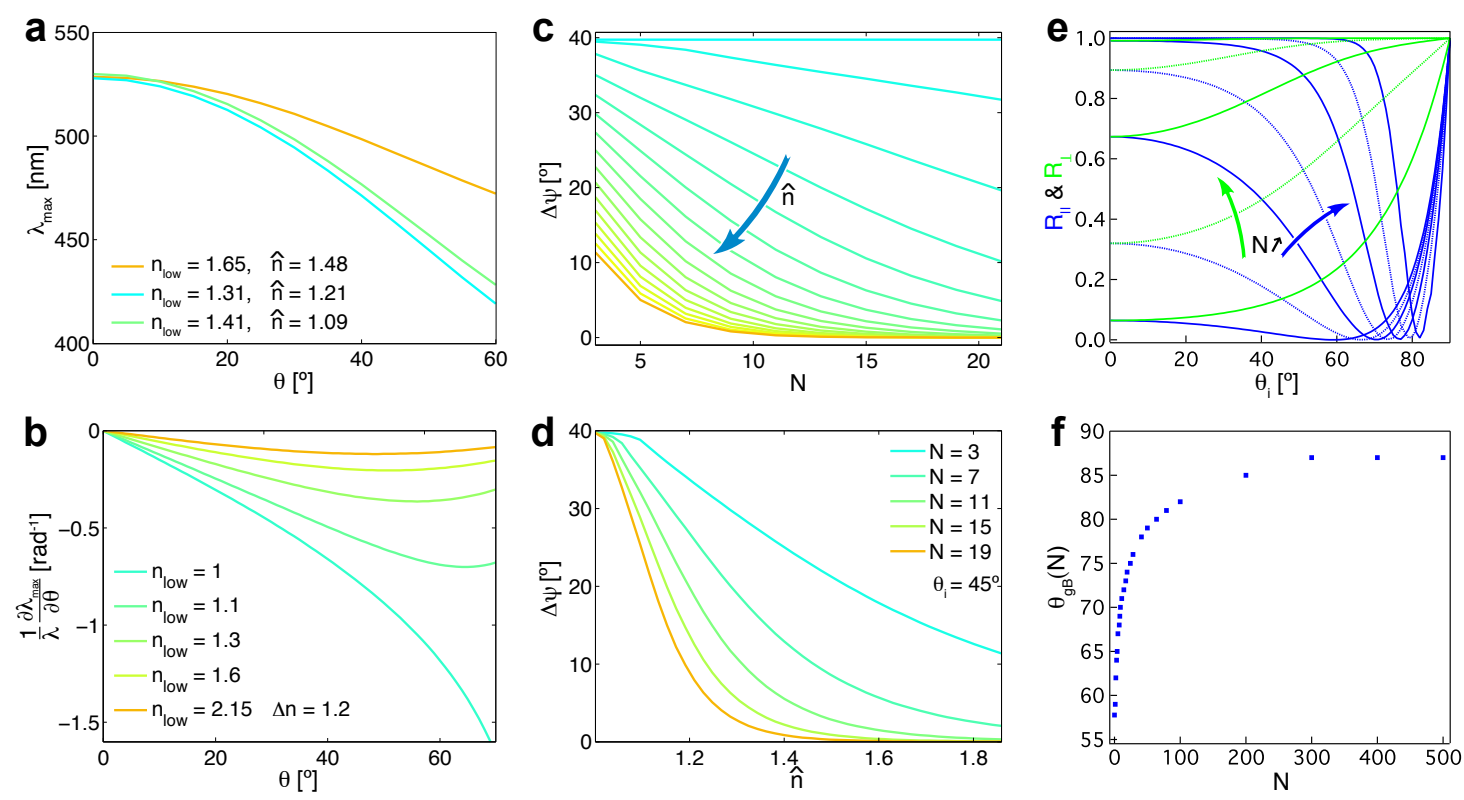

Figure 1.5: Peak wavelength and polarisation. a) Peak wavelength $\lambda_{\max }$ as a function of light incidence angle $\theta_{0}$ for varying refractive indices $n_{\text {low }}$ of the optically thinner material in the multilayer stack. b) Variation of peak wavelength as a function $\theta_{0}$ for different refractive indices $n_{\text {low }}$. c) Rotation of light polarisation after reflection for $\theta_{0}=45^{\circ}$ as a function of layer number $N$ and refractive index contrast $\hat{n}$. Incident light is polarised by $\psi=45^{\circ}$. d) Polarisation variation for $\theta_{0}=45^{\circ}, \psi=45^{\circ}$ as a function of refractive index contrast $\hat{n}$ for different layer numbers $N$. e) $R_{\|}$(blue) and $R_{\perp}$ (green) as a function of incidence angle $\theta_{0}$ for increasing layer number. $\mathbf{f}$ ) Variation of the generalised Brewster angle $\theta_{\mathrm{gB}}(N)$ at which $R_{\|}$is minimal, as a function of layer number.

layer. The difference between $R_{\|}$and $R_{\perp}$ vanishes for a large number of layers in the stack.

Increasing the refractive index contrast $\hat{n}$ leads to an increase in reflectivity (Fig. $1.4 \mathrm{c})$. The increase is more pronounced for perpendicularly-polarised light but for high contrasts the difference between $R_{\perp}$ and $R_{\|}$vanishes (Fig. $1.4 \mathrm{~d}$ ).

For increasing angles of light incidence $R_{\perp}$ increases monotonically towards unity while $R_{\|}$first decreases, reaching a minimum at the generalised Brewster angle $\theta_{\mathrm{gB}}$ of the multilayer stack [9], before it increases again.

The change in reflectance peak wavelength $\lambda_{\max }$ is more pronounced if the refractive index of the optically thinner material is lower (Fig. 1.5 a,b). For increasing layer number and increasing refractive index contrast, the variation in polarisation angle 
$\psi$ that the light incurs upon reflection decreases (Fig. $1.5 \mathrm{c}, \mathrm{d}$ ), provided the incidence angle $\theta_{0}$ is kept constant. The generalised Brewster angle $\theta_{\mathrm{gB}}$, at which the reflectivity for parallel polarised light $R_{\|}$drops drastically, increases as the number of layers is increased (Fig. 1.5 e,f). Provided the angle of incidence of unpolarised light matches $\theta_{\mathrm{gB}}(N)$ of the multilayer, high reflectivity can be achieved, while the reflected light is strongly polarised.

In general, a multilayer structure has to be tailored to its specific purpose by using appropriate materials and by suitably adapting the film thicknesses. Some guidelines based on the above presented discussions are given in Table 1.1.

\begin{tabular}{|c|c|c|c|c|c|c|c|c|}
\hline \multicolumn{4}{|c|}{ Desired optical properties } & \multicolumn{5}{|c|}{ Design requirements } \\
\hline Reflectivity & $\Delta \lambda$ & $\frac{\partial \lambda}{\partial \theta_{0}}$ & $\Delta \psi$ & $N$ & $\hat{n}$ & $n_{\text {low }}$ & $d_{i}$ & $\theta_{0}$ \\
\hline high & high & - & - & moderate $(>10)$ & high & - & $\frac{\lambda_{\max }}{4 n_{i}}$ & - \\
\hline high & low & - & - & high & low & - & $\frac{4 \lambda_{\max }}{4 n_{i}}$ & - \\
\hline high & low & high & - & high & low & $\simeq 1$ & $\frac{\lambda \lambda_{\max }}{4 n_{i}}$ & - \\
\hline high & high & low & - & high & high & high & $\frac{\lambda \lambda_{\max }}{4 n_{i}}$ & - \\
\hline$R_{\perp}$ high & - & - & high & low & low & - & $\frac{4 n_{\max }}{4 n_{i}}$ & $\theta_{\mathrm{gB}}(N)$ \\
\hline
\end{tabular}

Table 1.1: Optical properties of multilayer stacks depending on their design where $\Delta \lambda$ is the reflectance peak-width, $\theta_{0}$ the light incidence angle, $\Delta \psi$ the change in polarisation upon reflection, $\mathrm{N}$ the layer number, $\hat{n}$ the refractive index contrast, $n_{\text {low }}$ the lower refractive index in the multilayer and $d_{i}$ the thickness of the individual layers with refractive index $n_{i}$. "-" signifies "of minor relevance".

\subsection{Band-gaps of one-dimensional photonic crystals}

A photonic crystal is a material with refractive index varying periodically (on a length scale comparable to the wavelengths of light) in one, two or all three spatial dimensions. Multilayer stacks are often called one-dimensional photonic crystal structures due to their periodicity perpendicular to the layer planes. The term "photonic crystal" was inspired by Eli Yablonovitch and Sajeev John in 1987, who joined the tools of classical electromagnetism and solid-state physics to introduce the concepts of omnidirectional 
photonic band gaps in two and three dimensions. Since then, not only the well-studied Bragg reflectors and filters with band-gaps in one dimension, but especially two- and three-dimensional photonic structures used for "molding the flow of light" [4] have received considerable research interest. The band-gaps of a photonic structure are the frequency ranges for which light cannot propagate through this structure.

A common treatment of one-dimensional infinitely extended photonic crystals is based on a plane wave expansion technique. The discussion of this approach is focussed on photonic crystals consisting of lossless, linear dielectrics with no internal current sources and with magnetic permeability $\mu_{0} \approx 1$. This does not include metals, or nonlinear materials with optical absorption. Nevertheless, it provides excellent insight in the properties of photonic crystals and is appropriate for most of the optical structures found in nature. At the end of this section, the band diagrams of three different multilayer material systems, which are relevant to this thesis, are calculated.

The Maxwell equations that describe the link between a light wave's electric field $\vec{E}(\vec{r}, t)$ and its magnetic field $\vec{H}(\vec{r}, t)$ can be written in the following form

$$
\begin{aligned}
\nabla \cdot \vec{H}(\vec{r}, t) & =0 & \nabla \times \vec{E}(\vec{r}, t) & =-\frac{1}{c} \frac{\partial \vec{H}(\vec{r}, t)}{\partial t} \\
\nabla \cdot \varepsilon(\vec{r}) \vec{E}(\vec{r}, t) & =0 & \nabla \times \vec{H}(\vec{r}, t) & =\frac{\varepsilon(\vec{r})}{c} \frac{\partial \vec{E}(\vec{r}, t)}{\partial t},
\end{aligned}
$$

where $\varepsilon(\vec{r})$ is the periodically varying dielectric function of the photonic crystal and $c$ is the speed of light.

Electric or magnetic fields can in principle be decomposed into time-harmonic Fourier modes. Consequently, we can limit this discussion to the treatment of time-harmonic field patterns

$$
\begin{aligned}
\vec{H}(\vec{r}, t) & =\vec{H}(\vec{r}) \mathrm{e}^{\mathrm{i} \omega t} \\
\vec{E}(\vec{r}, t) & =\vec{E}(\vec{r}) \mathrm{e}^{\mathrm{i} \omega t}
\end{aligned}
$$


for which Maxwell's equations are given by

$$
\begin{aligned}
& \nabla \cdot \vec{H}(\vec{r}, t)=0 \quad \nabla \times \vec{E}(\vec{r}, t)=\quad-\frac{\mathrm{i} \omega}{c} \vec{H}(\vec{r}, t) \\
& \nabla \cdot \varepsilon(\vec{r}) \vec{E}(\vec{r}, t)=0 \quad \nabla \times \vec{H}(\vec{r}, t)=\varepsilon(\vec{r}) \cdot \frac{\mathrm{i} \omega}{c} \vec{E}(\vec{r}, t)
\end{aligned}
$$

From this equation system the wave equations for the magnetic and the electric field can be deduced.

$$
\begin{aligned}
\nabla \times\left(\frac{1}{\varepsilon(\vec{r})} \cdot \nabla \times \vec{H}(\vec{r})\right) & =\left(\frac{\omega}{c}\right)^{2} \vec{H}(\vec{r}) \\
\frac{1}{\varepsilon(\vec{r})} \cdot \nabla \times(\nabla \times \vec{E}(\vec{r})) & =\left(\frac{\omega}{c}\right)^{2} \vec{E}(\vec{r})
\end{aligned}
$$

These equations essentially represent two eigenvalue problems

$$
\begin{aligned}
\mathbf{O}_{H} \vec{H}(\vec{r}) & =\left(\frac{\omega}{c}\right)^{2} \vec{H}(\vec{r}) \\
\mathbf{O}_{E} \vec{E}(\vec{r}) & =\left(\frac{\omega}{c}\right)^{2} \vec{E}(\vec{r})
\end{aligned}
$$

with the operators

$$
\begin{aligned}
\mathbf{O}_{H} \vec{H}(\vec{r}) & =\nabla \times\left(\frac{1}{\varepsilon(\vec{r})} \cdot \nabla \times \vec{H}(\vec{r})\right) \\
\mathbf{O}_{E} \vec{E}(\vec{r}) & =\frac{1}{\varepsilon(\vec{r})} \cdot \nabla \times(\nabla \times \vec{E}(\vec{r}))
\end{aligned}
$$

Both operators are linear and the operator $\mathbf{O}_{H}$ is Hermitian, which is not necessarily the case for the operator $\mathbf{O}_{E}$ [4]. The eigenvalues of Hermitian operators are real, and two eigenmodes $\vec{H}_{1}(\vec{r}), \vec{H}_{2}(\vec{r})$ with eigenvalues $\omega_{1} \neq \omega_{2}$ are orthogonal to each other, that is the inner product vanishes:

$$
\left(\vec{H}_{1}(\vec{r}), \vec{H}_{2}(\vec{r})\right) \equiv \int \vec{H}_{1}^{*}(\vec{r}) \cdot \vec{H}_{2}(\vec{r}) d \vec{r}=0
$$

In addition, the eigenmodes of a Hermitian operator can be classified by their symmetry properties and valuable information can be obtained about the symmetry of field modes, arising from the symmetry in the dielectric function $\varepsilon(\vec{r})$ of the material. 
In the following, the eigenvalue problem is solved for infinitely extended stacks of alternating layers made from two optically distinct, lossless dielectric materials with refractive indices $n_{1}, n_{2}$ and thicknesses $d_{1}, d_{2}$. This system has a high symmetry in one dimension and the direction of periodicity is chosen to be the $z$-coordinate of the associated reference system. Similar systems have already been investigated earlier in this chapter, using traditional approaches, such as the transfer matrix technique or Rouard's method. Here, the main focus is directed towards the analysis of the band structure of a layered system. This technique can be generalised to two- or threedimensional photonic crystals. The dielectric function $\varepsilon(\vec{r})$ of the infinite multilayer with layer thicknesses $d_{1}, d_{2}$ and unit cell length $p=d_{1}+d_{2}$ is given by

$$
\varepsilon(\vec{r})=\varepsilon(z)=\left\{\begin{array}{cc}
n_{1}: & m p-\frac{d_{1}}{2} \leq z<m p+\frac{d_{1}}{2} \\
n_{2}: & \left(m+\frac{1}{2}\right) p-\frac{d_{2}}{2} \leq z<\left(m+\frac{1}{2}\right) p+\frac{d_{2}}{2}
\end{array}\right.
$$

where $m$ is a positive number.

It can easily be shown that the operator $\mathbf{O}_{E}$ is hermitian ${ }^{1}$ for this particular dielectric function $\varepsilon(\vec{r})$. This allows us to draw conclusions about the symmetry of the fields in the multilayer structure based on the symmetry of the system. The symmetry argument is outlined below, followed by the application of the plane wave expansion technique to find the electric field distributions $\vec{E}(\vec{r})$ of the eigenmodes and the corresponding eigenfrequencies of $\mathbf{O}_{E}$. The corresponding magnetic field distributions $\vec{H}(\vec{r})$ can then be deduced from $\vec{E}(\vec{r})$ via Maxwell's equations ${ }^{2}$.

The multilayer system is translationally invariant for any displacement $\vec{\rho}=x \hat{x}+y \hat{y}$ in the plane of the layers, where $\hat{x}$ and $\hat{y}$ are unit vectors along the $x$ - and $y$-direction. That is $\varepsilon(\vec{r}+\vec{\rho})=\varepsilon(\vec{r})$. The amplitude of the eigenmodes of a system with translational invariance in $x$ - and $y$-direction can only be a function of the $z$-coordinate. The phase

\footnotetext{
${ }^{1}$ This is done by showing that the inner product $\left(\vec{E}_{1}(\vec{r}), \mathbf{O}_{E} \vec{E}_{2}(\vec{r})\right)=\left(\mathbf{O}_{E} \vec{E}_{1}(\vec{r}), \vec{E}_{2}(\vec{r})\right)$ for any vector fields $\vec{E}_{1}(\vec{r})$ and $\vec{E}_{2}(\vec{r})$.

${ }^{2}$ Working with the electric field rather than the magnetic field, is only justified by the fact, that the plane wave expansion technique is less cumbersome but the approach would be the same for the magnetic field.
} 
relationship between different points in the photonic crystal with the same $z$-coordinate is given by the wave vector component $\vec{k}_{\rho}=k_{x} \hat{x}+k_{y} \hat{y}$. Thus the eigenmodes have the form

$$
\vec{E}_{k_{\rho}}(x, y, z)=\mathrm{e}^{\mathrm{i} \vec{k}_{\rho} \vec{\rho}(x, y)} \cdot \vec{e}(z)
$$

where the field amplitude $\vec{e}$ is only dependent on the spatial variable $z$.

Due to the discrete symmetry of the system in $z$-direction, the eigenmodes must be periodic along the $z$-coordinate, that is

$$
\vec{E}(x, y, z+p)=\mathrm{e}^{\mathrm{i} \vec{k}_{\rho} \vec{\rho}(x, y)} \cdot \vec{e}(z+p)=\mathrm{e}^{\mathrm{i} \vec{k}_{\rho} \vec{\rho}(x, y)} \cdot \vec{e}(z)=\vec{E}(x, y, z),
$$

where $p$ is the period of the system. According to Bloch's theorem [10], the periodic wave function $\vec{e}(z)$ can be expressed by a product of a plane wave with a $z$-periodic function

$$
\vec{e}_{k_{z}}(z)=\mathrm{e}^{\mathrm{i} k_{z} z} \vec{u}_{k_{z}}(z)
$$

and consequently the eigenmodes of such a periodic system, also know as Bloch states, are given in general by

$$
\vec{E}_{\vec{k}}(\vec{r})=\mathrm{e}^{\mathrm{i} \vec{k} \vec{r}} \cdot \vec{u}_{\vec{k}}(\vec{r})
$$

For light travelling along the $z$-direction this simplifies to the scalar relation

$$
E_{k_{z}}(z)=\mathrm{e}^{\mathrm{i} k_{z} z} \cdot u_{k_{z}}(z)
$$

Bloch states with wave-vectors $k_{z}$ and $k_{z}+m \cdot \frac{2 \pi}{p}$ are identical from a physical point of view. Thus, the eigenvalues of these Bloch states, which represent the frequencies of the corresponding modes, must also be periodic in $k_{z}$, that is $\omega\left(k_{z}\right)=\omega\left(k_{z}+m \cdot \frac{2 \pi}{p}\right)$. The Brillouin zone is the region in a dispersion diagram $\omega(k)$ which contains only the non-redundant wave vectors $k_{z}$ in the range $-\frac{\pi}{p}<k_{z} \leq \frac{\pi}{p}$. The band structure of a photonic crystal is known, if the dispersion relation $\omega(k)$ in its Brillouin zone is determined. This can be done with a plane wave expansion technique.

Assuming for simplicity a wave with a field vector only along the $x$-direction, 
$\vec{E}(\vec{r})=E_{x}(\vec{r}) \cdot \hat{x}$, the wave equation

$$
\frac{1}{\varepsilon(\vec{r})} \cdot \nabla \times \nabla \times \vec{E}(\vec{r})=\frac{\omega^{2}}{c^{2}} \vec{E}(\vec{r})
$$

simplifies to

$$
\frac{1}{\varepsilon(\vec{r})}\left(-\frac{\partial^{2} E_{x}(\vec{r})}{\partial y^{2}}-\frac{\partial^{2} E_{x}(\vec{r})}{\partial z^{2}}\right)=\left(\frac{\omega}{c}\right)^{2} E_{x}(\vec{r})
$$

where $\varepsilon(\vec{r})$ is given by Equation 1.15. Fourier-expansion of the field and the inverse dielectric function $\frac{1}{\varepsilon}$ yields

$$
\begin{aligned}
E_{x}(z) & =\sum_{t=-\infty}^{\infty} \tilde{E}_{t} \mathrm{e}^{-\frac{2 \pi \mathrm{i} t}{p} z} \\
\frac{1}{\varepsilon(z)} & =\sum_{s=-\infty}^{\infty} \tilde{\varphi}_{s} \mathrm{e}^{-\frac{2 \pi \mathrm{i} s}{p} z}
\end{aligned}
$$

where $\tilde{E}_{t}$ and $\tilde{\varphi}_{s}$ are the Fourier coefficients of the field $E_{x}(z)$ and the admittance $\frac{1}{\varepsilon(z)}$, respectively. Inclusion of the Fourier expansions in the wave equation gives, after reorganisation and simplification, the eigenequation that has to be solved in order to determine the band diagram:

$$
\sum_{t=-\infty}^{\infty}\left(\frac{2 \pi t}{p}+k_{z}\right)^{2} \tilde{\varphi}_{s-t} \tilde{E}_{t}=\left(\frac{\omega}{c}\right)^{2} \tilde{E}_{s} .
$$

After symmetric truncation $\left(|t| \leq t_{\max },|s| \leq s_{\max }\right)$ of the Fourier series of $E_{x}(z)$ and $\frac{1}{\varepsilon(z)}$, this equation becomes an eigenvalue problem which can be written in the form

$$
\mathrm{O}_{s, t} \tilde{E}_{t}=\left(\frac{\omega}{c}\right)^{2} \tilde{E}_{s}
$$

with $\mathbf{O}_{s, t}$ being a $2 s_{\max }+1$ - by - $2 t_{\max }+1$ matrix with its elements $O_{s, t}$ given by

$$
O_{s, t}=\left(\frac{2 \pi t}{p}+k_{z}\right)^{2} \tilde{\varphi}_{s-t} .
$$


The Fourier coefficients $\tilde{\varphi}_{s-t}$ can be found by using the inverse Fourier transform

$$
\begin{aligned}
\tilde{\varphi}_{s-t} & =\frac{1}{p} \int_{-\frac{p}{2}}^{\frac{p}{2}} \frac{1}{\varepsilon(z)} \cdot \mathrm{e}^{\frac{2 \pi \mathrm{i}(s-t)}{p} z} d z \\
& =\frac{1}{p}\left(\int_{-\frac{p}{2}}^{-\frac{d_{1}}{2}} \frac{1}{n_{2}^{2}} \cdot \mathrm{e}^{\frac{2 \pi \mathrm{i}(s-t)}{p} z} d z+\int_{-\frac{d_{1}}{2}}^{\frac{d_{1}}{2}} \frac{1}{n_{1}^{2}} \cdot \mathrm{e}^{\frac{2 \pi \mathrm{i}(s-t)}{p} z} d z+\int_{\frac{d_{1}}{2}}^{\frac{p}{2}} \frac{1}{n_{2}^{2}} \cdot \mathrm{e}^{\frac{2 \pi \mathrm{i}(s-t)}{p} z} d z\right) \\
& =\frac{1}{n_{2}^{2}} \cdot \delta_{s-t}+\left(\frac{1}{n_{1}^{2}}-\frac{1}{n_{2}^{2}}\right) \cdot \operatorname{sinc}\left(\frac{\pi(s-t) d_{1}}{p}\right) .
\end{aligned}
$$
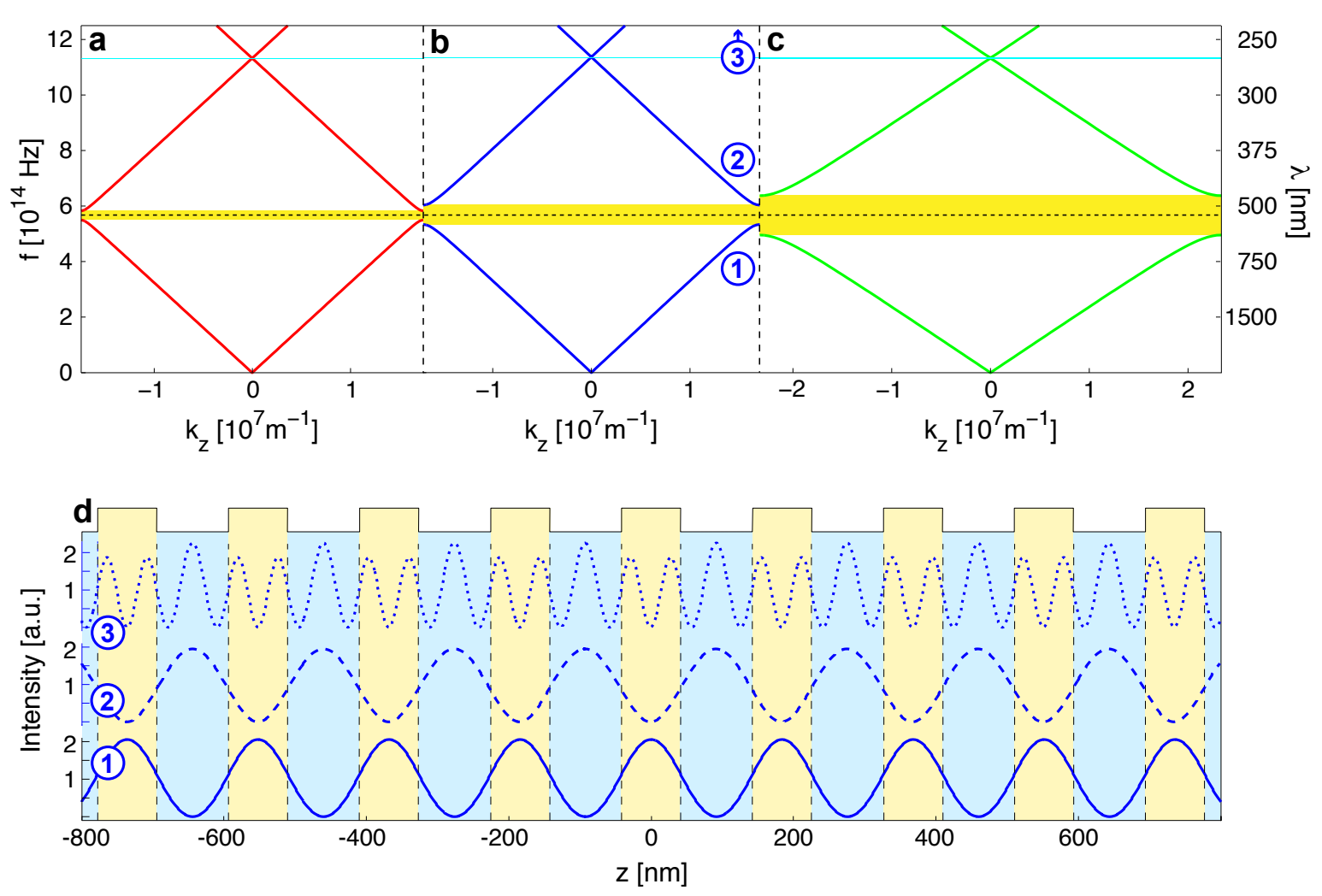

Figure 1.6: Band diagrams and field distributions of infinitely extended 1D photonic crystals. a)-c) The first Brillouin zone in the dispersion diagrams of three different multilayer structures for light propagation perpendicular to the layer planes. The corresponding refractive indices are shown in Table 1.2 together with the film thicknesses of the layers in each of the three infinite stacks, which were chosen in order to give a mid-gap wavelength of $530 \mathrm{~nm}$ (dashed black line) for all three material combinations. The refractive index contrast, $\hat{n}=\frac{n_{1}}{n_{2}}$ is increasing from left to right. a) $\hat{n}=1.09$, b) $\hat{n}=1.21$, c) $\hat{n}=1.48$. For each refractive index contrast, the first two bands are displayed. 1D photonic crystals always show band-gaps (here marked in yellow and blue) for wave propagation along the direction of periodicity; the size of the gaps depends on $\hat{n}$ (the gaps marked in blue are very small as the refractive index contrasts of the presented multilayers is not very large). d) Field distribution inside the infinite photonic crystal system with $\hat{n}=1.21$ for modes at the edges of the first three bands with their positions marked in the band diagram in (b). 


\begin{tabular}{|c|c|c|c|c|c|c|}
\hline & \multicolumn{3}{|c|}{ Constituent 1} & \multicolumn{3}{|c|}{ Constituent 2} \\
\hline & Material & $n_{1}$ & $\boldsymbol{d}_{1}[\mathrm{~nm}]$ & Material & $n_{2}$ & $\boldsymbol{d}_{2}[\mathrm{~nm}]$ \\
\hline 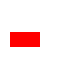 & PSPI & 1.54 & 86 & Polydimethylsiloxane & 1.41 & 94 \\
\hline - & Polystyrene & 1.59 & 83 & Teflon ${ }^{\circledR} \mathrm{AF}$ & 1.31 & 101 \\
\hline$\square$ & Titanium(II)oxide & 2.45 & 54 & Aluminium oxide & 1.65 & 80 \\
\hline
\end{tabular}

Table 1.2: Multilayer material combinations used for band-gap calculations. The colours in the first column correspond to the colours in Fig. 1.6. PSPI stands for polystyrene-polyisoprene triblock copolymer. Further information about the displayed materials can be found in Chapter 3.

By computational diagonalisation of the matrix $\mathbf{O}_{s, t}$, its eigenvectors $\tilde{E}$ and the corresponding eigenvalues $\left(\frac{\omega}{c}\right)^{2}$ can be determined. The eigenvectors $\tilde{E}$ can be introduced back into the Fourier expansion of $\vec{E}$ to find the field distribution. The eigenvalues give the dispersion diagram.

Each infinite multilayer stack of two optically distinct dielectrics $\left(n_{1} \neq n_{2}\right)$, has band-gaps. Their extent depends on the refractive index contrast of the constituent materials. Figure 1.6 shows the calculated band-gaps of multilayer stacks made from three different material combinations. The corresponding materials, their refractive indices and layer thicknesses are displayed in Table 1.2. Band-gaps are wider for larger refractive index contrasts. Figure $1.6 \mathrm{~d}$ shows the field distributions of three modes lying at the edge of the Brillouin zone $\left(k_{z}=\frac{\pi}{p}\right)$ for a material combination with refractive index contrast $\hat{n}=1.21$. The position of these modes on the three lowest bands are marked in Figure $1.6 \mathrm{~b}$. For the minimisation of its energy, the electric field of the ground mode (1), tends to be located in the regions of high dielectric constant $\varepsilon$. The modes with higher frequencies than the ground mode tend to concentrate their field in the high dielectric material obeying the constraint of remaining orthogonal to the modes that have a lower frequency [4].

The band-diagrams shown in Figure 1.6 correspond to multilayer stacks that have been fabricated from the material combinations, displayed in Table 1.2. These structures are presented in detail in Chapter 4. 


\subsection{Multilayer resonance cavities}

One-dimensional multilayer resonance cavities, so called interference filters or Bragg filters, usually consist of a "defect" layer of $m \cdot \frac{\lambda}{2}$-thickness, introduced into a $\frac{\lambda}{4}$-multilayer stack (Fig 1.7, $m$ is a positive number). This arrangement allows the existence of localised states in the defect layer at frequencies $f=\frac{m c}{2 n_{j} d_{j}}$ within the band-gap of the quarter-wave stack. Here $n_{j}$ and $d_{j}$ are the refractive index and the thickness of the defect layer. Light with these frequencies can propagate through the structure, as it fulfils the resonance condition $m \frac{\lambda}{2}=n_{j} d_{j}$.
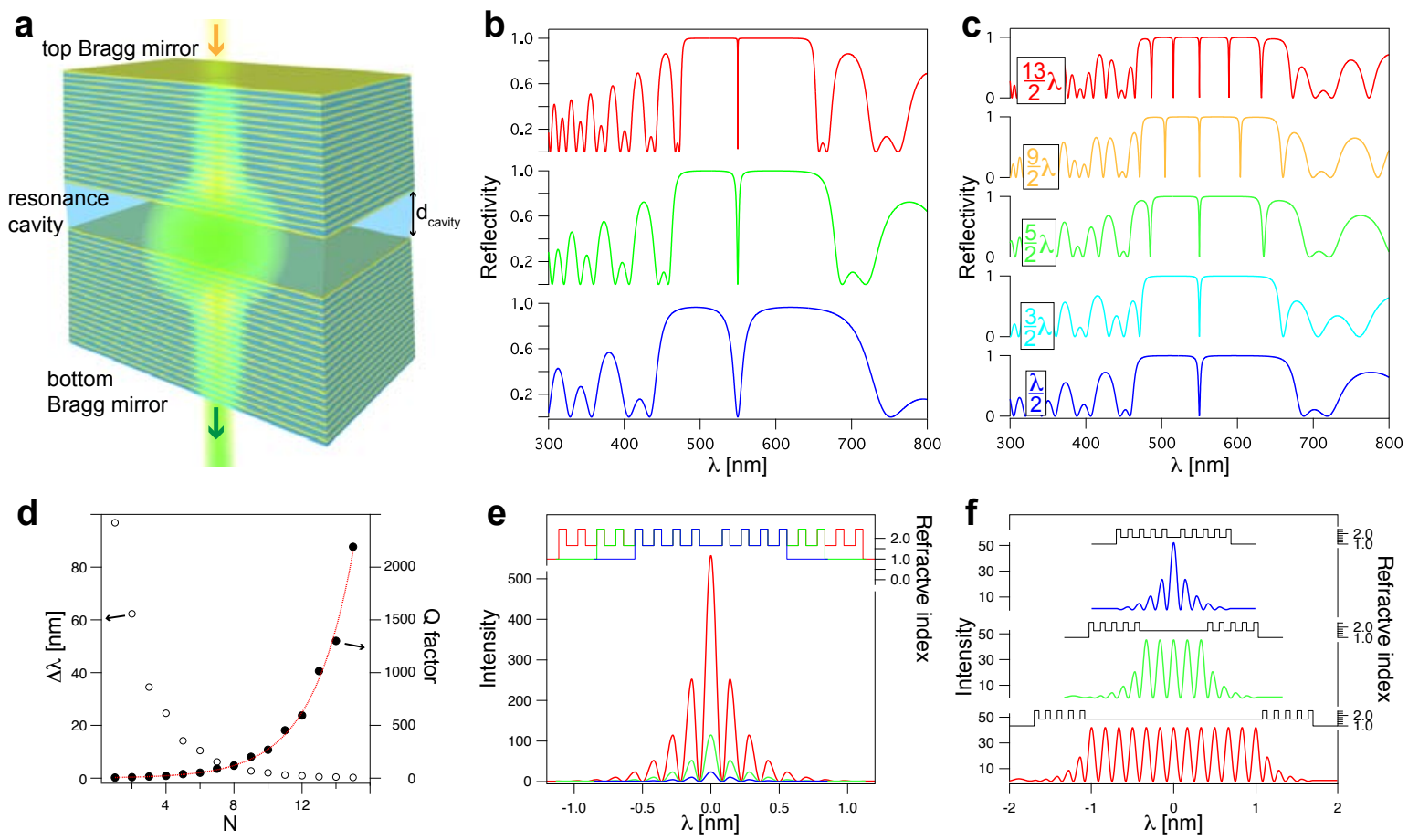

Figure 1.7: Multilayer interference filter. a) Schematic drawing of a filter with two $N$-layered Bragg mirrors enclosing a resonance cavity of thickness $d_{\text {cavity }}$. b) Reflectivity of a Bragg filter with a cavity layer thickness of $d_{\text {cavity }}=\frac{\lambda}{2}$ for increasing layer numbers $N$ in the two Bragg mirrors, 5 layers (blue), 9 layers (green), 15 layers (red). c) Reflectivity of Bragg filters with different cavity thicknesses in multiples of $\frac{\lambda}{2}$. d) Calculated bandwidth (white circles) and quality factor (black circles) of a $\frac{\lambda}{2}$-resonance cavity as a function of layer number in the top and bottom Bragg mirrors. e) Field distributions inside $\frac{\lambda}{2}$-resonance cavities enclosed by Bragg mirrors of layer number $N=7$ (blue), $N=11$ (green), $N=15$ (red). f) Field distributions inside a Bragg filter for varying cavity layer thicknesses, $d_{\text {cavity }}=\frac{\lambda}{2}$ (blue), $d_{\text {cavity }}=\frac{5 \lambda}{2}$ (green), and $d_{\text {cavity }}=\frac{13 \lambda}{2}$ (red). 
Bragg mirrors, made from titania and alumina layers with refractive indices $n_{\mathrm{TiO}_{2}}=2.45$, $n_{\mathrm{Al}_{2} \mathrm{O}_{3}}=1.65$ and film thicknesses of $d_{\mathrm{TiO}_{2}}=56 \mathrm{~nm}, d_{\mathrm{Al}_{2} \mathrm{O}_{3}}=83 \mathrm{~nm}$, have a peak reflectance wavelength of $\lambda \approx 550 \mathrm{~nm}$ (for more details on the materials see Chapter 3 ). The reflection characteristics of a free-standing Bragg filter consisting of a cavity layer of alumina with thickness $d_{\text {cavity }}$ between two of the above described Bragg mirrors are displayed in Figure 1.7 as a function of defect layer thickness and layer number of the Bragg mirrors enclosing the cavity layer. The bandwidth of a multilayer resonance cavity decreases as the number of layers in the Bragg stacks increases (Fig. 1.7 d). This is expressed in the quality factor of a cavity $Q=\frac{\lambda_{\mathrm{pb}}}{\Delta \lambda}$ where $\lambda_{\mathrm{pb}}$ and $\Delta \lambda$ are the centre wavelength and the width of the pass-band. The $Q$-factor is a measure for the losses of field intensity that a light wave incurs in a cavity per field oscillation period due to scattering or adsorption. The higher the $Q$-factor, the smaller are these losses and the higher are the maximum field strengths that can build up in the cavity for a wave that satisfies the resonance condition.

\subsection{Diffraction from periodic surface structures}

Surfaces or materials with a lateral periodicity of the order of several wavelengths impose an amplitude and/or phase modulation on an incident light wave that can be detected in the transmitted and reflected wave part as a characteristic diffraction pattern. In the following, the discussion will concentrate on the reflection of light from a phase grating with high transparency and uniform material properties. The grating has a periodicity $p$ in the $x$-direction. It is uniform in the $y$-direction and its surface normal is oriented parallel to the $z$-direction (Fig. 1.8). Consider a spherical light wave with intensity $I_{0}\left(x_{0}, z_{0}\right)$ originating from the point $\left(x_{0}, z_{0}\right)$ travelling in the $x$-z-plane towards the grating situated in the " $z=0$ "-plane (Fig. 1.8, the $y$-dimension is not relevant for this discussion). A diffraction pattern, caused by the interaction of that wave with the grating is observed on a cylindrical screen with radius $L$ centered at the grating position and intensities $I(x, z)$ are detected at points $(x, z)$ on the screen. 


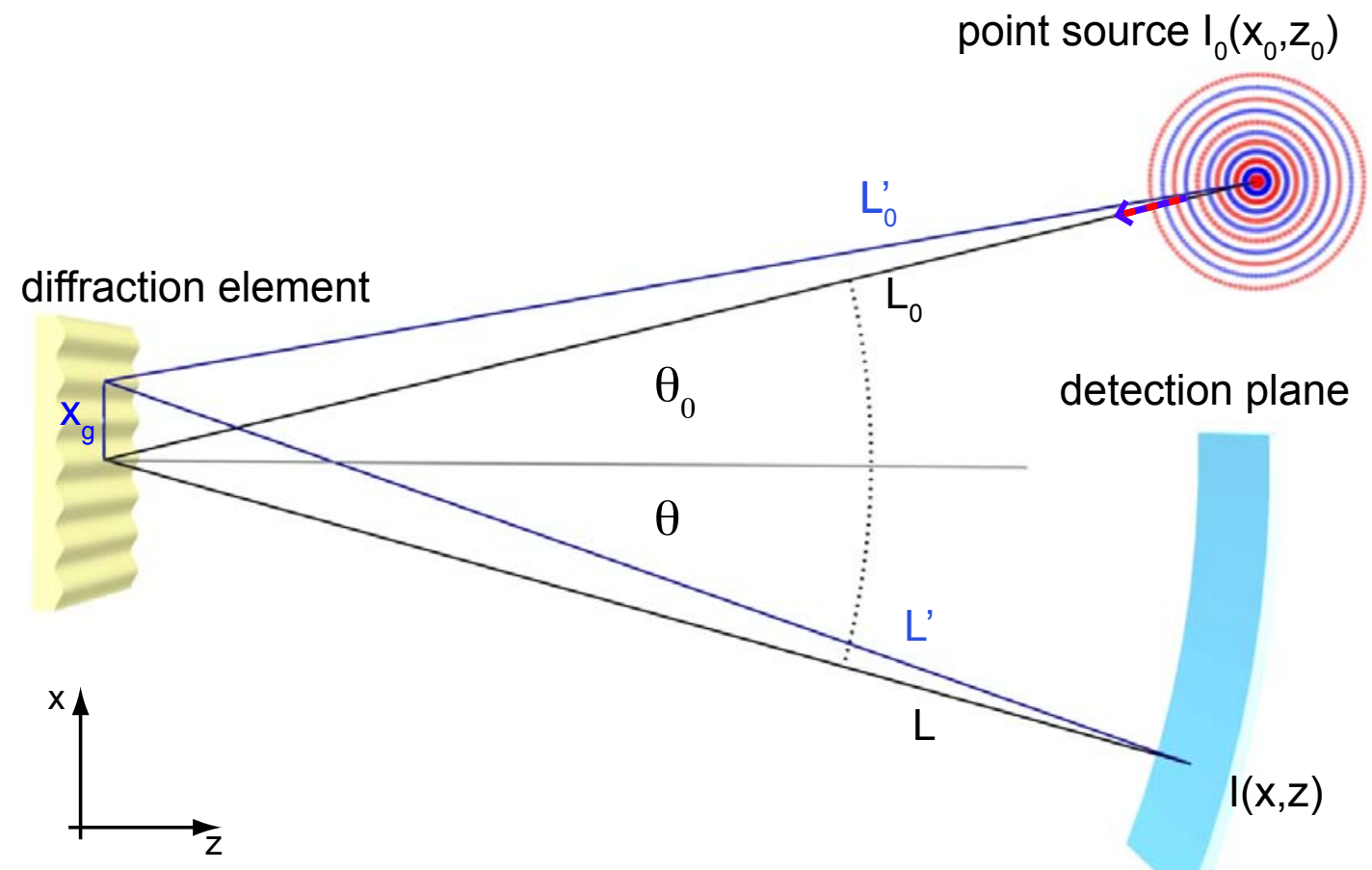

Figure 1.8: Geometry of the diffraction problem. Light from a point source at a distance $L_{0}$ is scattered from the grating and the diffraction pattern is detected on a screen at a distance $L$ from the grating.

\subsubsection{Diffraction in the Fraunhofer approximation}

If the distance $L_{0}$ of the light source to the diffracting object is large enough compared to the light wavelength $\lambda$, the waves incident on the grating can be treated as plane waves. If in addition the distance $L$ of the observation point to the grating is large compared to the grating periodicity and the light wavelength, the observation point is said to be lying in the far-field and the diffraction problem can be treated in the Fraunhofer approximation [2].

The reflection function $F_{\mathrm{g}}$ of one periodic grating element is defined by

$$
F_{\mathrm{g}}\left(x_{\mathrm{g}}\right)=\frac{V\left(x_{\mathrm{g}}\right)}{V_{0}\left(x_{\mathrm{g}}\right)}
$$

where the disturbance $V\left(x_{\mathrm{g}}\right)$ of the light wave caused by the grating element is weighted 
by the default disturbance $V_{0}\left(x_{\mathrm{g}}\right)=\mathrm{e}^{\mathrm{i} k \sin \theta_{0} \cdot x_{\mathrm{g}}}$ when no grating is present. For a phase grating, consisting of a transparent dielectric, that is observed in reflection, $F_{\mathrm{g}}\left(x_{\mathrm{g}}\right)$ satisfies $\left|F_{\mathrm{g}}\right|=r$, where $r$ is the Fresnel reflection coefficient of the dielectric material. The function $F_{\mathrm{g}}\left(x_{\mathrm{g}}\right)$ depends on the particular form of the grating unit cell.

The Fraunhofer approximation of the Kirchhoff-Fresnel diffraction integral, which describes the field amplitude observed at point $(x, z)$ on the screen, after light has been diffracted from one periodic grating element, is given by

$$
S\left(x_{0}, z_{0}, x_{1}, z_{1}\right)=-\frac{\mathrm{i}\left(\cos \theta+\cos \theta_{0}\right)}{2 \lambda L_{0} L} \int_{-\frac{p}{2}}^{\frac{p}{2}} F_{g}\left(x_{g}\right) \mathrm{e}^{\mathrm{i} k\left(L^{\prime}\left(x, z, x_{g}\right)+L_{0}^{\prime}\left(x_{0}, z_{0}, x_{g}\right)\right)} d x_{g}
$$

where the approximation

$$
\begin{aligned}
& L_{0}^{\prime 2}=\left(x_{0}-x_{g}\right)^{2}+z_{0}^{2} \approx L_{0}-\frac{x_{0}}{L_{0}} x_{g} \\
& L^{\prime 2}=\left(x-x_{g}\right)^{2}+z^{2} \approx L-\frac{x}{L} x_{g}
\end{aligned}
$$

together with

$$
\begin{aligned}
\frac{x_{0}}{L_{0}} & =-\sin \theta_{0} \\
\frac{x}{L} & =\sin \theta
\end{aligned}
$$

leads to the reflection function of one periodic element of the grating

$$
S\left(\theta, \theta_{0}\right)=-\frac{\mathrm{i}\left(\cos \theta+\cos \theta_{0}\right)}{2 \lambda L_{0} L} \mathrm{e}^{\mathrm{i} k\left(L_{0}+L\right)} \int_{-\frac{p}{2}}^{\frac{p}{2}} F_{g}\left(x_{g}\right) \mathrm{e}^{-\mathrm{i} k\left(\sin \theta-\sin \theta_{0}\right) x_{g}} d x_{g}
$$

The field distribution $U$ at point $(x, z)$, otherwise defined by its distance $L$ from the grating centre and the angle $\theta$ to the surface normal of the grating, follows from summation over all the contributions of the $N$ individual grating unit cells as 


$$
\begin{aligned}
U\left(\theta, \theta_{0}\right) & =S\left(\theta, \theta_{0}\right) \cdot \sum_{n=0}^{N-1} \mathrm{e}^{-\mathrm{i} n p k\left(\sin \theta-\sin \theta_{0}\right)} \\
& =S\left(\theta, \theta_{0}\right) \cdot \frac{1-\mathrm{e}^{-\mathrm{i} N p k\left(\sin \theta-\sin \theta_{0}\right)}}{1-\mathrm{e}^{-\mathrm{i} p k\left(\sin \theta-\sin \theta_{0}\right)}}
\end{aligned}
$$

The field, observed at an angle $\theta$, is essentially a superposition of plane waves resulting from the unit cells of the grating, each of them modulated in the same way by the reflection function of a grating unit cell.

The intensity observed in distance $L$ at an angle $\theta$ is then given by

$$
I\left(\theta, \theta_{0}\right)=\left|U\left(\theta, \theta_{0}\right)\right|^{2}=\left|S\left(\theta, \theta_{0}\right)\right|^{2} \cdot\left(\frac{\sin \frac{N p k\left(\sin \theta-\sin \theta_{0}\right)}{2}}{\sin \frac{p k\left(\sin \theta-\sin \theta_{0}\right)}{2}}\right)^{2} .
$$

The results of this approximation for large angles of light incidence $\theta_{0}$ and detection $\theta$ have to be considered with caution as the implied simplifications might not necessarily apply. By suitable normalisation $\frac{S\left(\theta, \theta_{0}\right)}{S_{0}\left(\theta, \theta_{0}\right)}$, where $S_{0}\left(\theta, \theta_{0}\right)$ is the reflection function of a $100 \%$-reflective, phase-conserving plane mirror, the pre-factor of the integral giving the reflection function $S\left(\theta, \theta_{0}\right)$ of one periodic element vanishes.

This approximation was employed to model the diffraction from a simple square phase grating. The results were compared to MEEP Finite-Difference Time-Domain simulations of the same square grating to test the reliability of the MEEP algorithm, before applying it to model diffraction from structures with more complex unit cell profiles. The results of this comparison are presented in Figure 1.10 below, after a short introduction in the FDTD simulations of diffraction with MEEP.

\subsubsection{Finite-Difference Time-Domain modelling of diffraction}

MEEP, a Finite-Difference Time-Domain (FDTD) simulation package, is widely used for computational electromagnetism [7]. MEEP has been programmed by D. Roundy, M. Ibanescu, P. Bermel and S. G. Johnson at the Massachusetts Institute of Technology. 
MEEP solves Maxwell's field equations in a particular dielectric structure and its vicinity, by discretising time and space on a finite rectangular grid with a spatial resolution $\Delta x$ and a temporal resolution $\Delta t<\frac{n_{\min }}{\sqrt{N_{\mathrm{D}}}} \Delta x[7]$. Here, $n_{\min }$ is the minimum refractive index $\left(n_{\min }=1\right.$ for air) and $N_{\mathrm{D}}$ is the number of relevant system dimensions. For the discretisation of the field equations with second-order accuracy, MEEP uses Yee lattices, storing different field components for different grid locations [11]. If all field components at a specific point on the lattice need to be known, they can be obtained by interpolation. In general this approach is not problematic but it can cause some inaccuracies if fields are read out very close to dielectric boundaries, across which they might be discontinuous.

The MEEP package can be controlled via $\mathrm{C}++$ libraries or a scheme script, tailored to the specific application. For calculations of diffraction, a $\mathrm{C}++$ interface has been implemented in the context of this thesis. The $\mathrm{C}++$ algorithm can acquire refractive index maps from image files. These image files might be refractive index maps that have been created, based on contrasts in SEM or TEM pictures (for instance shown in Fig. 1.9).

Diffraction from periodic micro-structures can be simulated in two dimensions by looking at diffraction from a unit cell, while implementing periodic boundary conditions on the sides of the simulation volume to account for the periodicity of such a grating (Fig. $1.10 \mathrm{a}$ ). The top and the bottom of the simulation volume are delimited by "perfectly matched layers" (PML). These boundary layers absorb any incident waves, preventing artefacts that could result from spurious interferences with re-entrant waves. Detectors are positioned at sufficient distances from the diffracting interface to avoid coupling with evanescent surface modes. A plane-wave Gaussian pulse is created at an
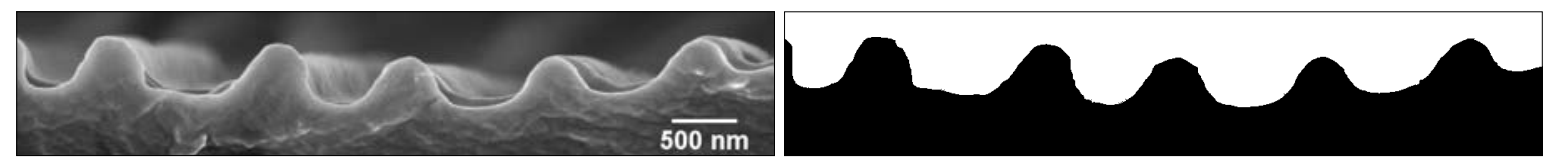

Figure 1.9: A grating structure, and its binary image for MEEP. Electron micrograph and a binarised image of the grating structure found on the surface of wild tulips. SEM image taken from the surface of Tulipa kolpakowskiana. 
appropriate distance from the grating interface (Fig. $1.10 \mathrm{~b}$ ). Depending on its width, the pulse contains a range of frequencies. This pulse is propagated through the structure in discrete time steps $\Delta t$ by solving Maxwell's equations at each point of the Yee lattice to acquire the relevant field values at this point. The reflected and transmitted fields (Fig. $1.10 \mathrm{c}$ ) are detected at the coordinates $y_{\mathrm{r}}$ and $y_{\mathrm{t}}$ in the unit cell for each time step $n$ giving $E_{\mathrm{r}}\left(x, y_{\mathrm{r}}, n \cdot \Delta t\right)$ and $E_{\mathrm{t}}\left(x, y_{\mathrm{t}}, n \cdot \Delta t\right)$, shown in Fig. $1.10 \mathrm{~d}$. Fourier transformation in time

$$
E_{\mathrm{r}, \mathrm{t}}(x, f)=\int_{0}^{t_{\max }} E_{\mathrm{r}, \mathrm{t}}(x, t) \cdot \mathrm{e}^{-\mathrm{i} 2 \pi f t} d t
$$

gives the electric fields as a function of position and frequency. Fourier transformation along the space coordinate $x$

$$
E_{\mathrm{r}, \mathrm{t}}\left(k_{x}, f\right)=\int_{-\frac{p}{2}}^{\frac{p}{2}} E_{\mathrm{r}, \mathrm{t}}(x, \omega) \cdot \mathrm{e}^{-\mathrm{i} k_{x} x} d x
$$

allows the determination of the electric fields as a function of wave vector and frequency. The integration boundaries for the second transformation are given by the unit cell width $p$.

Using the relations

$$
\begin{aligned}
\lambda & =\frac{c}{f}, \\
\sin \theta & =\frac{k_{x}}{k}=\frac{k_{x} c}{2 \pi f}, \\
I_{r, t} & =E_{r, t}^{*} \cdot E_{r, t}
\end{aligned}
$$

the intensity $I(\lambda, \theta)$ of light can be plotted as a function of the diffraction angle $\theta$, which is measured from the sample normal. Note that the incidence angle is $\theta_{0}=0$ for the results in Figure 1.10, which shows simulations of diffraction from a square-profile phase grating with $p=1.4 \mu \mathrm{m}$. The simulated reflected and transmitted intensities $I_{\mathrm{r}}^{\operatorname{sim}}(\lambda, \theta)$ and $I_{\mathrm{t}}^{\operatorname{sim}}(\lambda, \theta)$ are plotted in Figures $1.10 \mathrm{f}, \mathrm{h}$. For comparison, the reflected and transmitted intensities $I_{\mathrm{r}}^{\text {calc }}(\lambda, \theta)$ and $I_{\mathrm{t}}^{\text {calc }}(\lambda, \theta)$, which have been obtained by evaluation of the Fresnel-Kirchhoff diffraction formula in the Fraunhofer approximation (as described above), are displayed in Figures $1.10 \mathrm{~g}$, i. For the simple case of a 

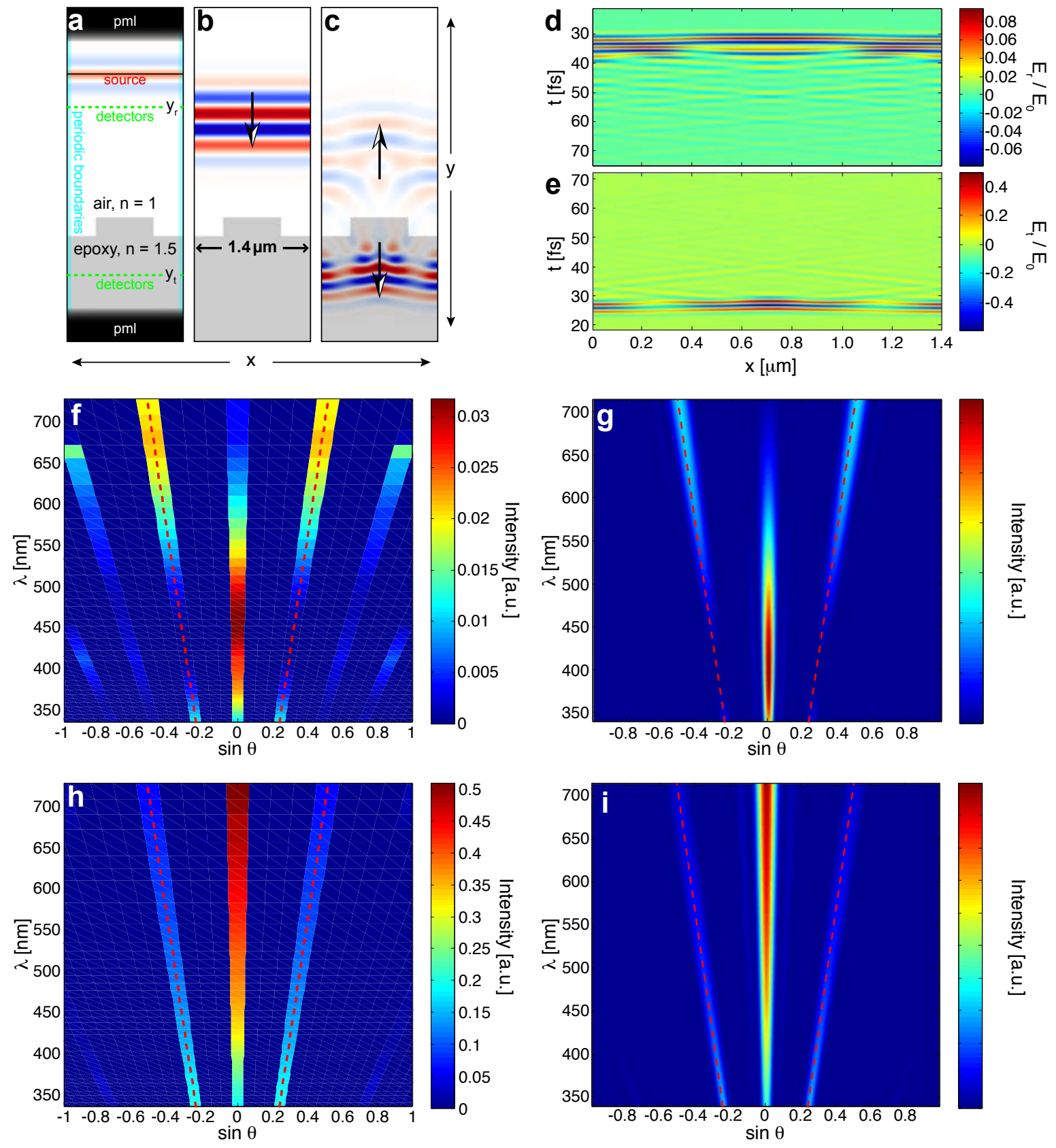

Figure 1.10: Diffraction grating simulation with MEEP. a) Representation of the computational volume displaying the refractive index map of a square phase grating unit cell, "perfectly matching layers", source and detector positions. b) Gaussian pulse of light with wavelengths ranging from 300 to $800 \mathrm{~nm}$ propagating towards the grating interface. c) Reflected and transmitted parts of the incident wave packet. d) and e) Field amplitudes measured in the planes of the reflection (d) and transmission (e) detector as a function of time and $x$-coordinate on the detector. There is good agreement between the diffraction patterns, observed in reflection for the FDTD simulations (f) and for calculations in the Fraunhofer approximation ( $(\mathbf{g})$. h) and i) show the diffraction patterns observed in transmission for the simulations and the scattering calculations in the Fraunhofer approximation. The red dashed lines in $(\mathbf{f}-\mathbf{i})$ represent the $1^{\text {st }}$ and $-1^{\text {st }}$ diffraction orders as a function of $\lambda$ and sine of $\theta$ determined by the grating equation $\lambda= \pm p \sin \theta$. 
square-profile phase grating, there is a good agreement between the data obtained in simulations and in approximative calculations for the intensities detected in the far field. FDTD simulations with MEEP could potentially be used to model diffraction from more complicated periodic geometries found in the form of regular striations on the petals of wild tulip species (Chapter 2.2).

These calculations were performed with the help of Moritz Kreysing, who pioneered the use of MEEP in the Biological and Soft Systems sector and the Nanophotonics group of the Cavendish Laboratory and played a leading role in realising the diffraction simulations. 



\section{References}

[1] Land, M. The physics and biology of animal reflectors. Prog. Biophys. Mol. Bio. 24, 75 (1972).

[2] Born, M. \& Wolf, E. Principles of Optics (Cambridge University Press, 2005).

[3] Allen Taflove, S. C. H. Computational electrodynamics: The Finite-Difference Time-Domain Method (Artech House, 1995).

[4] Joannopoulos, J. D., Johnson, S. G., Winn, J. N. \& Meade, R. D. Photonic crystals: Molding the flow of light (Princeton University Press, 2008).

[5] Heavens, O. Optical properties of thin solid films (Dover Publications, 1965).

[6] Johnson, S. G. \& Joannopoulos, J. D. Block-iterative frequency-domain methods for Maxwell's equations in a planewave basis. Opt. Express 8, 173-190 (2001).

[7] Oskooi, A. F. et al. MEEP: A flexible free-software package for electromagnetic simulations by the FDTD method. Computer Physics Communications 181, 687$702(2010)$.

[8] Kinoshita, S. \& Yoshioka, S. Structural colors in nature: The role of regularity and irregularity in the structure. ChemPhysChem 6, 1442-1459 (2005).

[9] Mahlein, H. Generalized Brewster-angle conditions for quarter-wave multilayers at non-normal incidence. J. Opt. Soc. Am 64, 647-653 (1974).

[10] Kittel, C. Introduction to solid state physics (John Wiley \& Sons, 1996).

[11] Yee, K. S. Numerical solution of initial boundary value problems involving maxwell's equations in isotropic media. IEEE T. Antenn. Propag. 14, 302-307 (1966). 



\section{Chapter $\mathscr{2}$}

\section{Structural colours in nature}

Bright, intense, pure colours play a vital role in flora and fauna. Animals often use colours to communicate with individuals of their own species. In this context colours can serve for agonistic behaviour between males fighting for the attention of a female or generally as a visually obvious criterion for sexual selection [1]. Colours also have a function in the interaction between different species. They can serve as camouflage (cryptic coloration) or as a warning sign (aposematic coloration) [2-4]. Many plants display strong and distinct colours on their blossoms to attract specific pollinators [5]. Some flowering plants also use particular reflecting structures to protect their blossoms and leaves against damaging UV light [6]. Nature offers an amazing variety of organisms which make use of structures to create bright colour and iridescence.

While pigment-based colours feature in the most abundant techniques used to create colour stimuli in nature, the brightest and most intense colours result from the interaction of light with complex structures on the micro- and nanoscale [7-12]. These structures are often made from materials with low inherent light absorption. Common physical mechanisms applied in nature's structural colours have been identified as multilayer interference, diffraction and coherent scattering often combined with specific absorption to increase the optical effect [13-17]. The best results are achieved with a fine interplay between regularity, providing the spectral filtering, and irregularity on different length scales, assuring a broader angular range of reflectance and thus higher visibility [18]. Structures in nature that fulfil a particular optical function, enforcing, for instance, strong reflectivity, bright colours, a particular light polarisation, a brilliant white, or extreme blackness, include simple, solid multilayers [18], microporous scattering films [16], periodically deformed, perforated multilayers [19], disordered cuticular filament networks [20], micro- light traps [21], conical micro-structures with internal 
chiral nano-structure [22], intercalated micro- and nano-structures on different length scales [23, 24], or gyroid structure-based photonic crystals [25, 26].

In this chapter, various photonic systems found in the animal kingdom that serve as role models for biomimetic work on structural colours are presented along with studies of diffraction structures on the flower petals of several plants. Floral photonic structures have been rarely investigated in detail, although recent research suggests that structural colours might also play a role in the plant world [27-29].

\subsection{Structural colours in the animal kingdom}

Although the structural colours of many birds, insects, mammals [30], and marine animals have been studied, the focus here lies on four species of different insect families that are the most relevant role models for the present thesis. These insects from the orders Coleoptera and Lepidoptera are the beetle Chrysochroa fulgidissima, the moth Chrysiridia rhipheus and butterflies of the genii Papilio and Morpho. The main structure responsible for the colours observed in these animals are multilayer arrangements with varying complexity. The beetle Chrysochroa fulgidissima displays a simple flat multilayer on its wing cases, while the moth Chrysiridia rhipheus and the $P a$ pilio and Morpho butterflies possess increasingly complex multilayer configurations to create stunning colour effects. Colour creation caused by diffractive surface elements will be studied on the example of floral structures. For details on other complex natural two- or three-dimensional photonic structures that cause bright colours, brilliant whiteness or perfect blackness the interested reader is referred to excellent articles in [18, 20, 21, 31-37].

\subsubsection{Simple multilayers - the Japanese jewel beetle}

Many insects of the order Coleoptera display impressive structural colours, which often have a metallic appearance [38]. The wood-boring beetle Chrysochroa fulgidissima, 

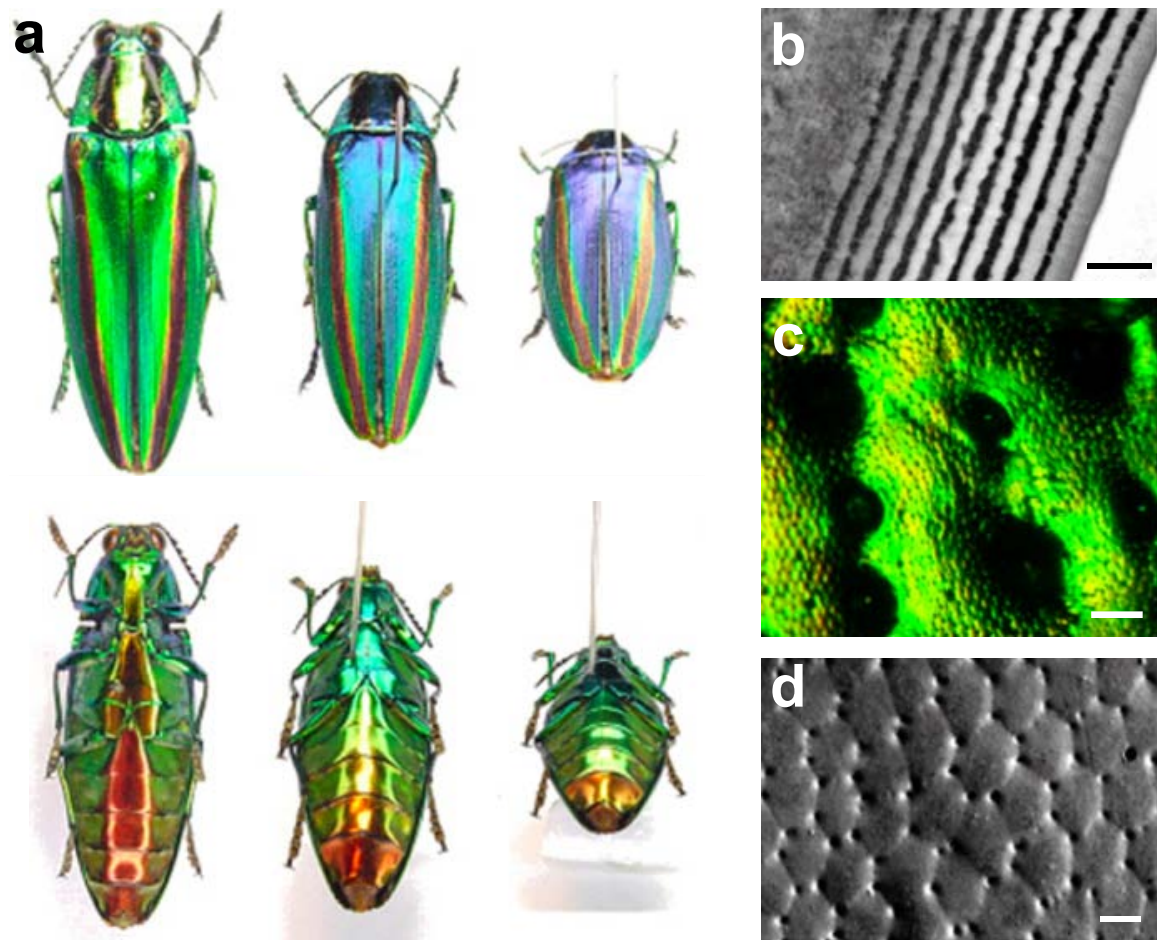

Figure 2.1: Chrysochroa fulgidissima - The Japanese Jewel Beetle. a) The elytra and the ventral side of this beetle display bright and iridescent colours that show a strong angular dependence. b) A multilayer stack with appropriate thicknesses and refractive indices of the constituent films causes interference of light and strong spectral selectivity for the reflected colour, scale bar $400 \mathrm{~nm}$. Irregular deformations of the multilayer - c) optical micrograph, scale bar $\sim 100 \mu \mathrm{m}$, and d) scanning electron micrograph, scale bar $10 \mu \mathrm{m}$ - assure scattering and the visibility of the colour over a wide angular range. All pictures are reproduced from [39].

from the family of Buprestidae, better known as the "Japanese jewel beetle", is found in the woods and forests of Japan during summer. The shell of the beetle shows a remarkable metallic iridescence on its elytra ${ }^{1}$ and on its ventral side (Fig. 2.1 a). The structure responsible for the bright iridescent colours is a multilayer arrangement in the epicuticle ${ }^{2}$ layer of the beetle shell, shown in the transmission electron microscope image in Figure $2.1 \mathrm{~b}$. This layer structure is made up of $\sim 20$ alternating layers with refractive indices $n_{1}=1.5$ and $n_{2}=1.7$ [18]. Irregular structures on the surface of the beetle shell (Fig. 2.1 c, d) assure that the beetle's strong colour appears not only in the direction of specular reflection but can also be observed from other angles.

\footnotetext{
${ }^{1}$ The elytra are the hardened forewings of beetles that protect the hindwings, which are used for flying.

${ }^{2}$ The epicuticle is the outermost portion of the exoskeleton of an insect.
} 


\subsubsection{Complex multilayer arrangements in butterfly and moth structures}

Multilayer arrangements of higher complexity, than those seen in Chrysochroa fulgidissima, are found on the wing scales of the moth Chrysiridia rhipheus and butterflies of the genii Papilio and Morpho. The wings of most animals classified in the insect order Lepidoptera are covered by small, regularly arranged scales with intricate fine-structure.

A optical classification of photonic Lepidoptera scale structures has been proposed by P.Vukusic and H. Ghiradella [31, 40] and extensive studies of the scale development have been presented by H. Ghiradella [41-43] (Fig. 2.2). The classification distinguishes between reflecting elements based on their structural nature and their position on or in the scales. Type I structures consist of cuticle-air multilayers implemented in the scale ridges. Type II elements encompass multilayer arrangements within the scale body and

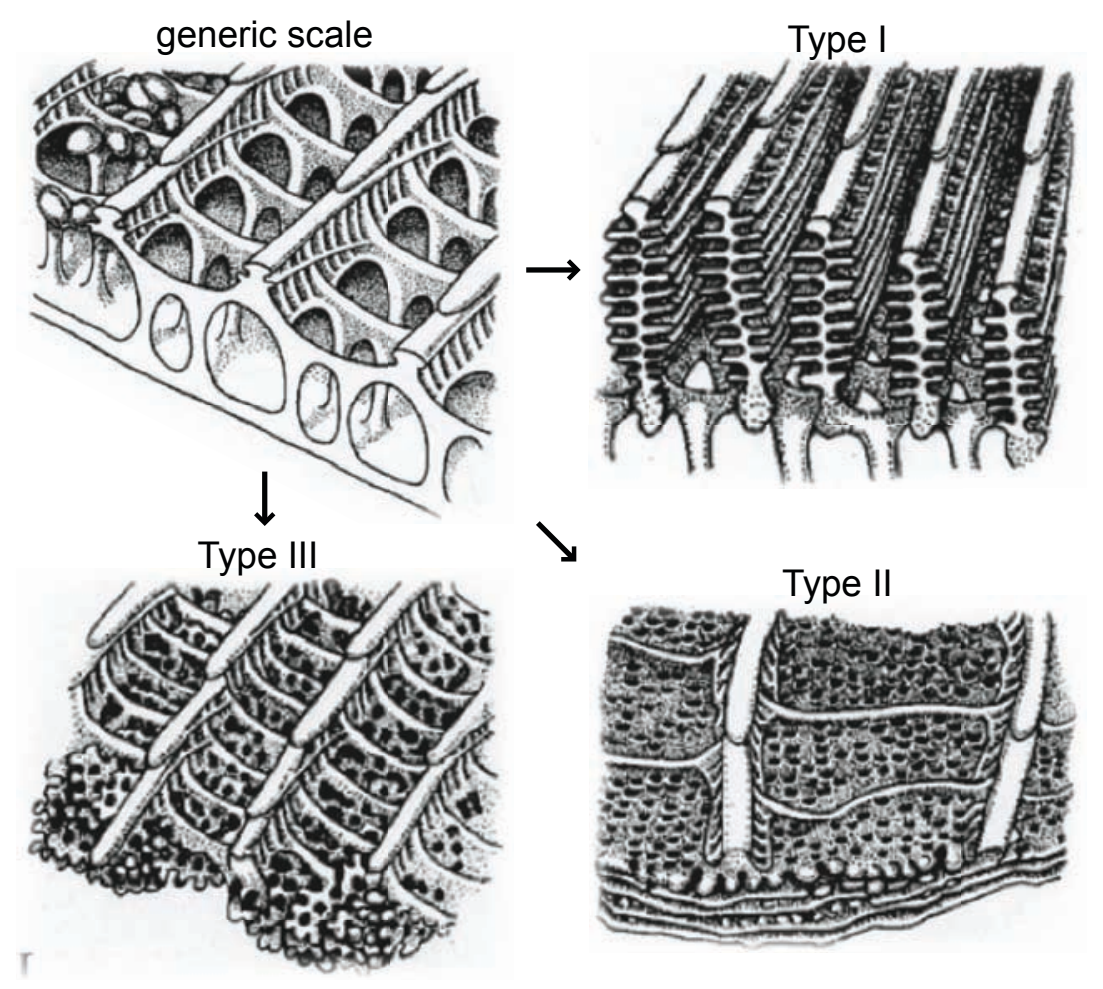

Figure 2.2: Structural classification of iridescent Lepidoptera scales. This schematic drawing displays the microstructures found on the wings scales of iridescent moths or butterflies. Reprinted with permission from H. Ghiradella and Wiley-Liss, (C) 1998 . 
type III structures comprise other specialised photonic systems [31]. The functionality of scale structures within this classification is investigated, in the following, for the case of different moth and butterfly species.

\section{Enhanced reflectivity in the moth Chrysiridia rhipheus - using air as a multilayer material}

The Madagascan moth Chrysiridia rhipheus, better known as the "sunset moth", is a diurnal species from the family Uraniidae. It displays bright green coloured areas on its wings together with two symmetric spots on the lower wing pair, which change colour from violet in the centre to red, orange and finally yellow on the outside of the spots, appearing like a beautiful sunset (Fig. 2.3 a). Each single wing scale in the "sun spot" reflects bright and intense colours (Fig. 2.3 b, c, d).

The colours result from the interaction of light with the intricate fine-structure on the wing scales. Two different kinds of scales are found on the wings of the sunset moth (Fig. 2.3 e). The first kind of scale, which causes the colours, has typical dimensions of about $300 \times 100 \mu \mathrm{m}$ (Fig. $2.4 \mathrm{a}$ ). These scales display small parallel, regularly spaced, micro-ribbed ridges running along the surface of the scales (Fig. $2.4 \mathrm{~b}$ ). The cross-section of one scale reveals a stack of equally thick cuticle lamellae separated by columnar spacers of a well-defined height (Fig. 2.4c,d). Interference of light in these lamellar multilayer stacks causes the bright colours [45]. Variations in the spacing and/or the thickness of the cuticular lamellae lead to a change in the reflected colour. In addition these scales are curved along the long axis. This causes multiple reflections of light from different scales. The scale curvature is the origin of the textured appearance of the bright wing areas on photos. Only the parts along the scales, which are oriented in the right direction with respect to the light source, reflect light back into the eye of the observer, the camera or the microscope objective (Fig 2.3 b, c). The scale curvature also has the additional effect that light angularly incident on a scale is highly polarised upon reflection from one or several scales [46].

Underneath the first type of scales described above and in the dark regions of the moth 
wing lies a different kind of scales. They appear entirely black under the microscope and are thought to function as an absorbing under-layer to eliminate light which has passed through the multilayer scales, thereby avoiding spurious reflections in unwanted spectral ranges. These scales have a different structure with more closely spaced ridges on the scale surface, stabilised by small struts (Fig. 2.5). At first glance it seems that
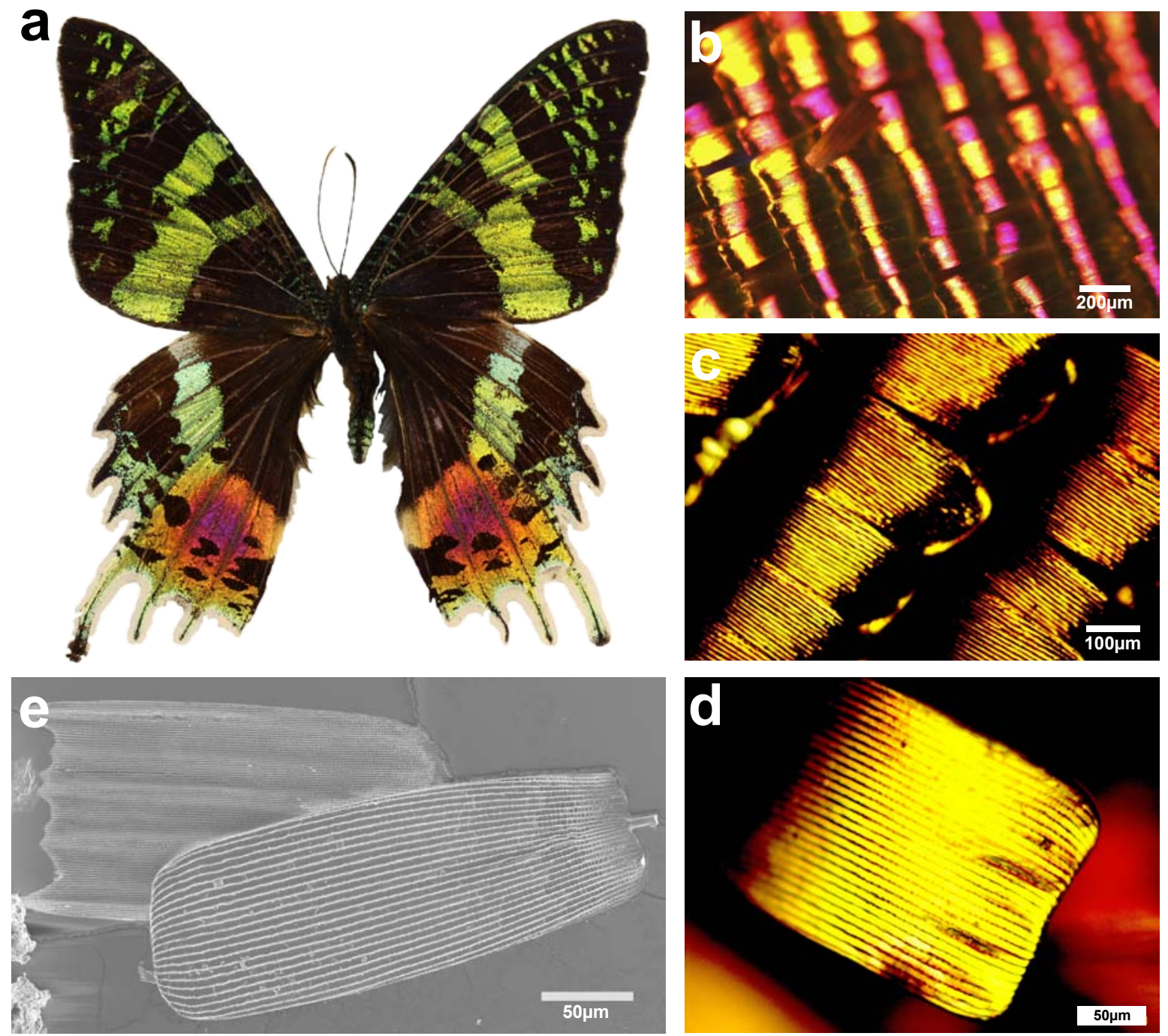

Figure 2.3: Chrysiridia rhipheus - the Madagascan "sunset" moth. a) The wings of this moth are covered in colourful scales reflecting green, yellow, red and violet light in different regions of the wings. The scales are highly curved so that the observer only perceives light reflected from part of the scales, which creates the impression of texture due to the juxtaposition of bright and dark regions (b, c). Two different kinds of scales are found on the wings of the moth, strongly reflecting ones, which cause the bright colours ( $\mathbf{d}$ and $\mathbf{e}$, right) and the dark ones, which provide absorption in the black wing parts (e, left). 

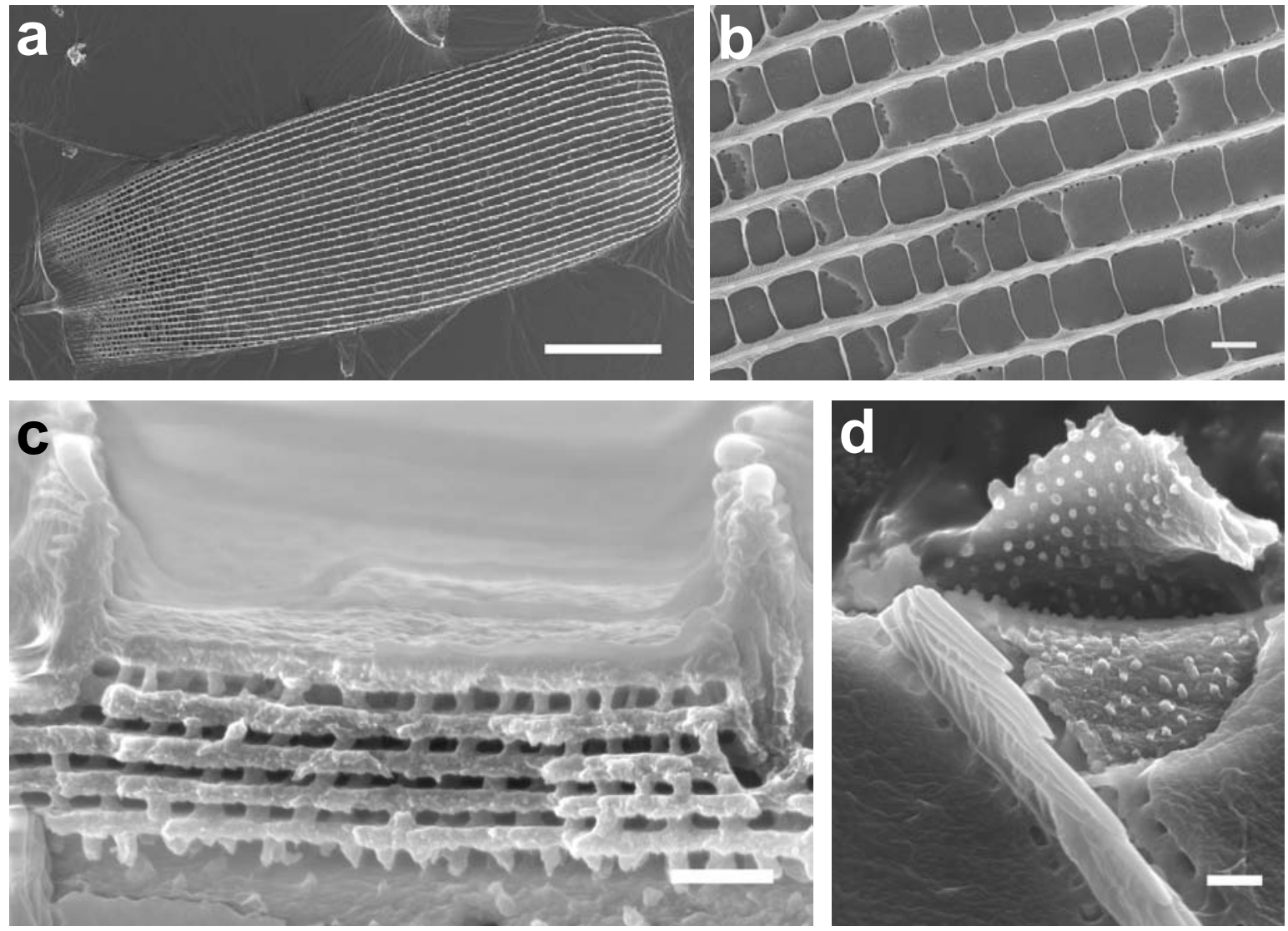

Figure 2.4: A scale with type II micro-structure. a) The colourful scales have dimensions of about $300 \times 100 \mu \mathrm{m}$, scale bar $50 \mu \mathrm{m}$. b) These scales are spanned by small parallel micro-ribbed ridges, scale bar $2 \mu \mathrm{m}$. c) The colour arises from a regular multilayer arrangement of cuticle layers held apart at a well-defined distance by, d) roughly hexagonally arranged cuticle pillars, scale bars $500 \mathrm{~nm}$.

the whole design of these scales is adjusted for maximum rigidity at minimum weight but in addition, they might also fulfil another purpose. Similar structures on the scales of the butterfly Papilio ulysses have been shown to assist in confining the incident light in the pigment-containing ${ }^{3}$ scale body which enhances the absorption drastically, thereby causing nearly-perfect structurally-assisted blackness [21].

In conclusion, the main features of the sunset moth are the multilayer of perforated cuticle nano-scale lamellae and the overall micro-scale curvature of the scales.

\footnotetext{
${ }^{3}$ Absorption in Lepidoptera scales is usually associated with melanin incorporated in the scale structure but other pigments can be found accumulated in granulae on the bottom of the scales [31].
} 

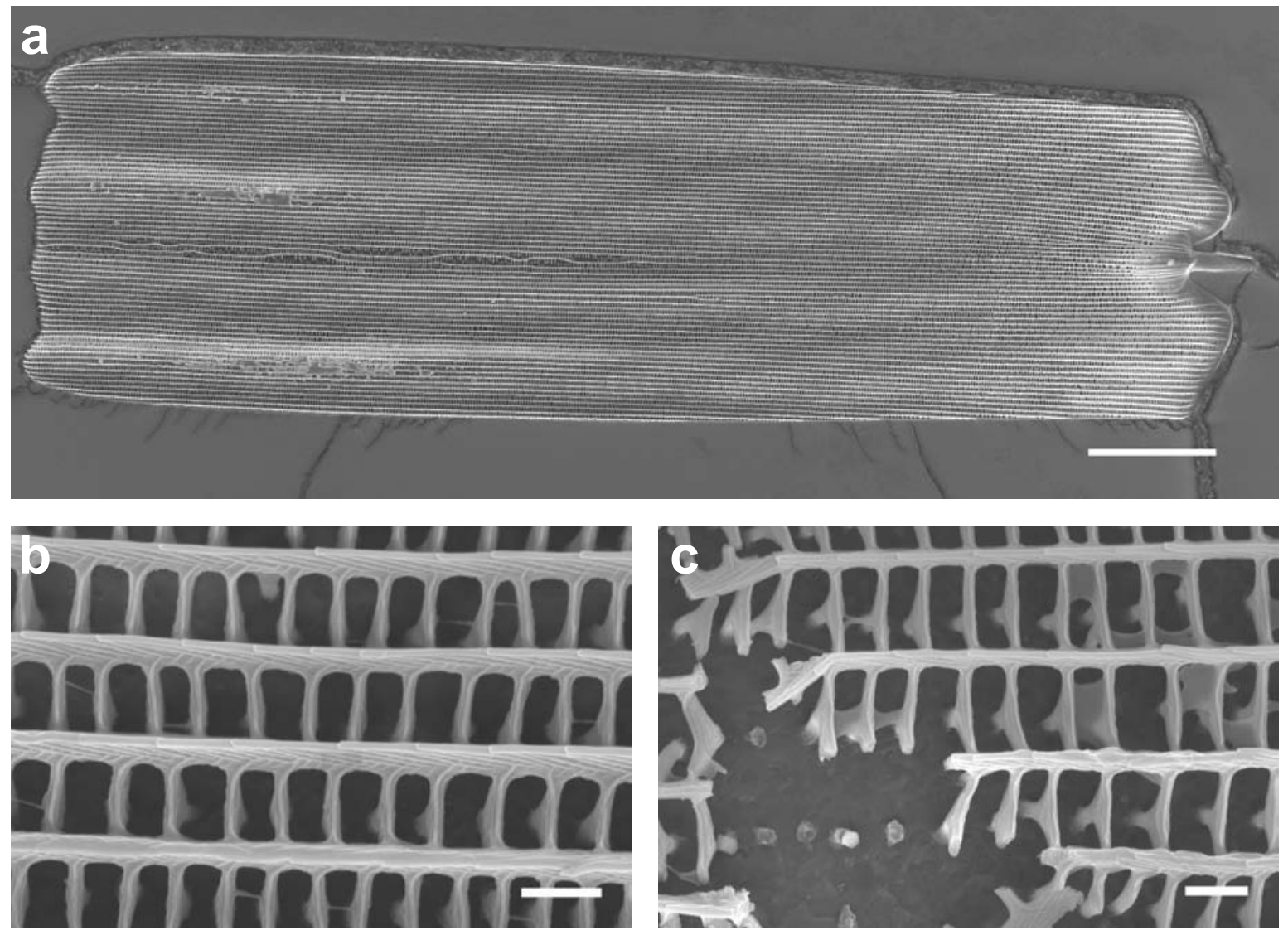

Figure 2.5: A scale with very low reflectivity. a) These black scales have similar dimensions as the multilayer scales of typell, scale bar $30 \mu \mathrm{m}$. b) In contrast to the colourful type II scales, their surface is spanned by a higher number of ridges, lying closer together and held apart by small struts which also anchor the ridges in the continuous base layer (c) of the otherwise hollow scale, scale bars $1 \mu \mathrm{m}$.

\section{Sculpted multilayer structures - Papilio palinurus and Papilio blumei}

The Indonesian swallowtail butterflies Papilio palinurus and Papilio blumei of the family Papilionidae display bright green patches on their wing pairs. Papilio blumei also shows characteristic blue spots on its lower wing tips (Fig. 2.6a). The bright green wing areas are covered in green scales of ca. $300 \mu \mathrm{m} \times 100 \mu \mathrm{m}$ in length and width. Closer inspection of the wing scales reveals the origin of the green colour. It arises from a juxtaposition of blue and yellow-green light reflected from different microscopic regions on the wing scales (Fig. 2.6 c, Fig. $2.7 \mathrm{a}, \mathrm{b}$ ). These are separated by only a couple of $\mu \mathrm{m}$ from each other and are thus not resolvable by the human eye. The top layer of colourful scales is backed by a second layer of black scales, possibly absorbing any light 

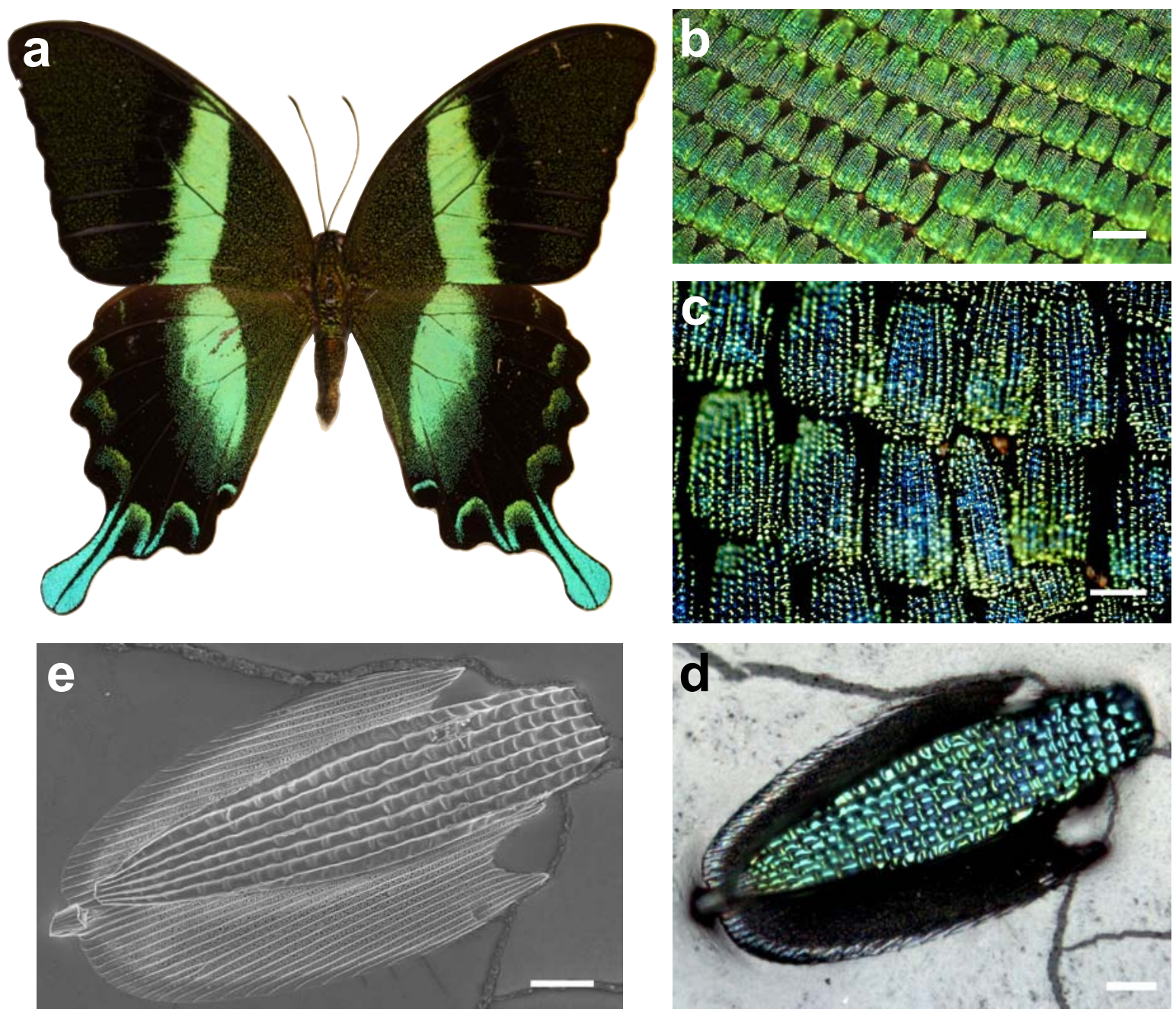

Figure 2.6: Papilio blumei - Colour mixing on the micro-scale. a) Papilio blumei, the green swallowtail butterfly. b) Green scales on the wings, scale bar $200 \mu \mathrm{m}$, (c) that show differently blue and yellow-green coloured regions at higher magnification, scale bar $100 \mu \mathrm{m}$. d), e) The colour scales are arranged on top of black scales with a different surface structure, scale bars $20 \mu \mathrm{m}$.

that passes through the first layer of scales avoiding spurious reflections which could reduce the colour contrast and compromise the bright green appearance.

The colour scales exist with and without ridges running parallel along the scales (Fig. $2.7 \mathrm{c}, \mathrm{d}$ ). They belong to the type II scales. The surface of the scales is marked by concavely shaped surface corrugations running along the scale in an ordered fashion (Fig. 2.7e,f). The centre of these concavities appear bright green or yellow in reflection while the edges reflect blue light (Fig. 2.7 b, left). Placing a scale between crossed polarisers allows the light coming from the concavity centres to be extinguished, while 

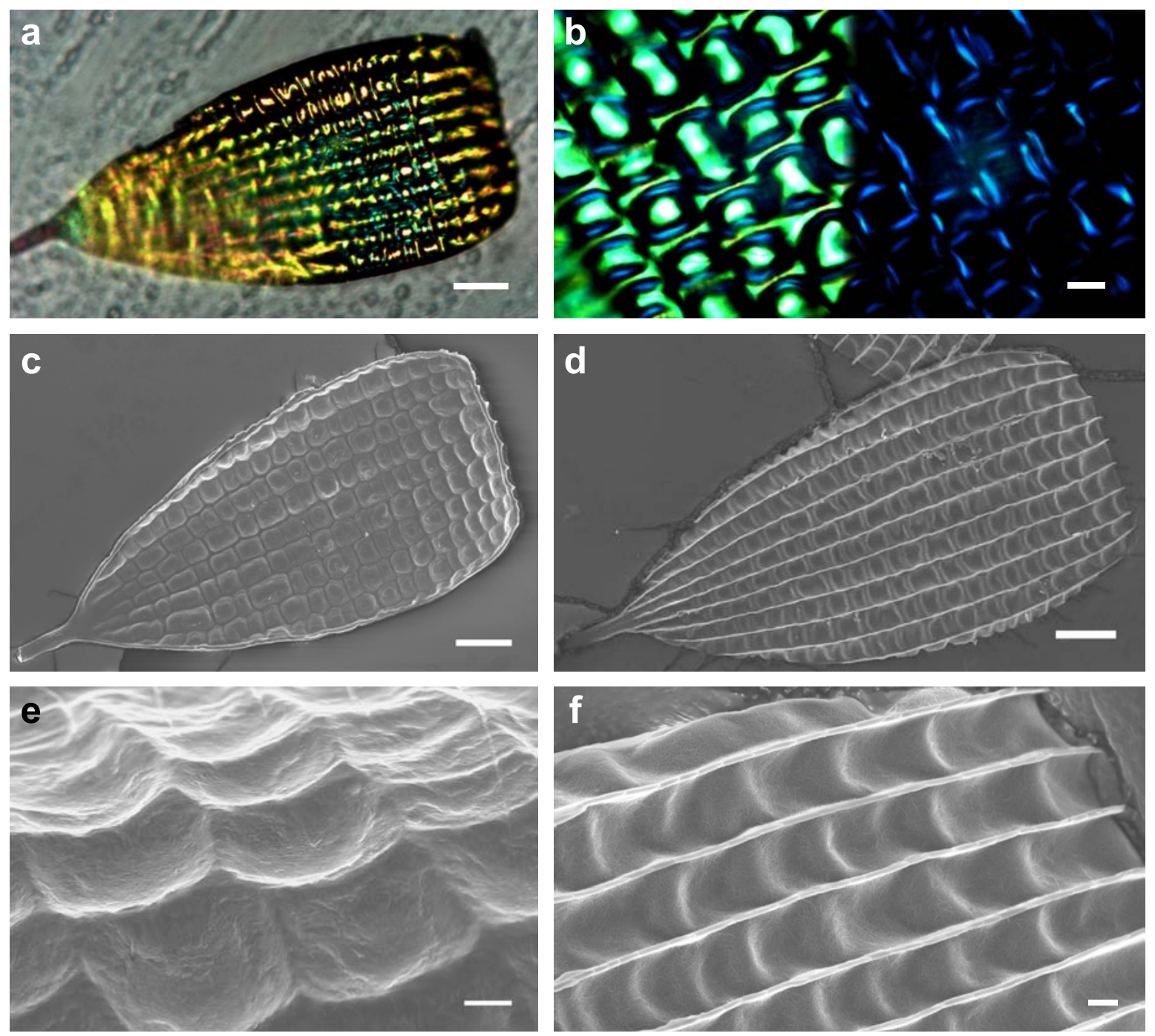

Figure 2.7: Wing scales of Papilio blumei. a) Single scale of the butterfly, scale bar $20 \mu \mathrm{m}$. b) High magnification reflection images from the scale surface under illumination with unpolarised light (left) and with the scale placed between crossed polarisers, scale bar $5 \mu \mathrm{m}$. c) Coloured scales show concavely shaped regularly arranged surface deformations running aligned along the scale, separated by small ridges (d) on some of the scales, scale bars $20 \mu \mathrm{m}$. e), f) Concavities on the scales with and without ridges, scale bars $2 \mu \mathrm{m}$.

blue light coming from the edges is detected (Fig. $2.7 \mathrm{~b}$, right). This effect, resulting from the sculpted multilayer cladding of the concavities on the scale surface, has been explained in the literature $[19,47]$ and will be examined more closely in Chapter 6.

This colour production mechanism suggests a clever way of providing leaf-green camouflage for protection against predators with polarisation insensitive eyes, while allowing communication with conspecifics on another colour channel (blue). Polarisation sen- 
sitive vision has already been seen for other insects but this hypothesis is still to be proven for Papilio butterflies [19, 48, 49].

\section{Fine-structured “christmas-tree"-like ridges on the scales of Morpho rhetenor}

The male Morpho rhethenor butterfly of the family Nymphalidae displays a strikingly vivid blue iridescent colour on its wings, which is reportedly visible across extremely large distances [8] (Fig 2.8 a). This outstanding colour raised the interest of scientists early on in the study of colour-initiating structures, making this butterfly and related species of the genus Morpho some of the most studied cases of natural structural colour $[7,15,50-53]$. The purpose of the butterfly's blue iridescent colour is thought to be high
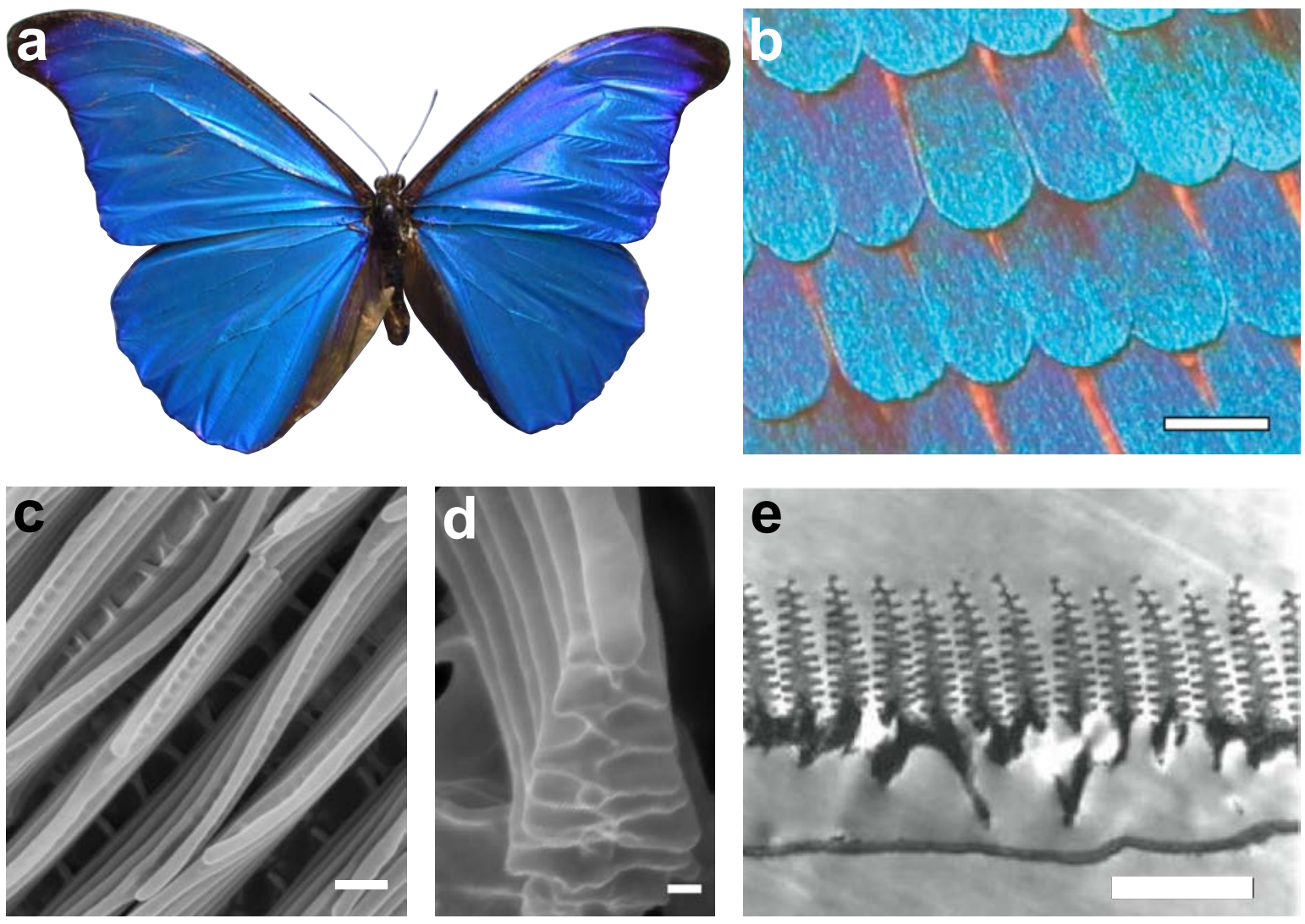

Figure 2.8: Photonic structures on the typel scales of Morpho rhetenor a) The butterfly Morpho rhetenor. b) Scales on the wings, scale bar $100 \mu \mathrm{m}$. c) The scales are spanned by ridges, scale bar $500 \mathrm{~nm}$. d) Cross-section through one of the ridges, scale bar $100 \mathrm{~nm}$. e) TEM section through a scale, showing the cuticle-air multilayers built into the ridges, scale bar $1.5 \mu \mathrm{m}$. Pictures taken from www.nano.ecs.soton.ac.uk (a) and [31] (b, e). 
visibility for conspecific communication, especially for agonistic display. Male butterflies use their bright, flashing blue wings to mark their territory and scare off potential male rivals in search of the less colourful female butterflies. The wing scales (Fig 2.8 b) are covered by regularly spaced ridges (Fig $2.8 \mathrm{c}$ ), which display an intricate 'christmastree'-like cross-sectional fine-structure (Fig $2.8 \mathrm{~d}$, e), classified as type I. This is one of the most sophisticated colour producing structures encountered in nature. The finestructure of each ridge effectively represents an air-cuticle multilayer with controlled cuticle thickness and layer spacings, providing high refractive index contrast, thus allowing for high reflectivity in the blue. The iridescent appearance and shimmer caused by multilayer reflection is intensified by the regular arrangement of scale ridges, which have a spacing of $\sim 800 \mathrm{~nm}$ and act partly as a diffraction grating [15]. Irregularity,

arising from slight variations in spacing between the ridges, the randomly appearing tilt of the ridges with respect to each other, and the varying orientation of the scales on the wing surface enhances the visibility of the striking colour in a broad angular range above the wing surface.

\subsection{Floral diffractive structures}

Natural structural colours in the animal kingdom have been investigated in great detail. In contrast, very little research has been devoted to the study of structural colours in the plant world, although recent work suggests that several plants use micro- and nano-structures which create particular optical effects. In many cases, such as for the blue spikemoss Selaginella Willdenowii [29, 54], little is known about the purpose of photonic structures on the plant surface. We have recently shown that striations on the flower petal surface of Tulipa kaufmanniana, Tulipa kolpakowskiana, and Hibiscus trionum can act as diffraction gratings [27]. This is discussed in the following section. Based on investigations of the flower species Mentzelia lindleyi, the last part of this chapter focusses on another potential benefit of petal striations, enhanced reflectivity in the ultra-violet (UV). 


\subsubsection{Diffraction and iridescence from striations on flower petals}

Recent research suggests that not only animals but also some plants use iridescence and structural colours for their purposes. We identified iridescence in flowers of Hibiscus trionum and the wild growing tulip species, Tulipa kaufmanniana and Tulipa kolpakowskiana (Fig. 2.9) and demonstrated that iridescence is generated through diffraction gratings that might be widespread among flowering plants. Although iridescence might be expected to increase attractiveness, it might also compromise target identification because the object's appearance will vary depending on the viewer's perspective. We found that bumblebees (Bombus terrestris, Fig. 2.9) learn to disentangle flower iridescence from colour and correctly identify iridescent flowers despite their continuously changing appearance. This ability is retained in the absence of cues from polarised light or ultraviolet reflectance associated with diffraction gratings.

Biological iridescence results from various mechanisms, including multilayered materials, crystalline inclusions and surface diffraction gratings [48, 55-59]. Diffraction
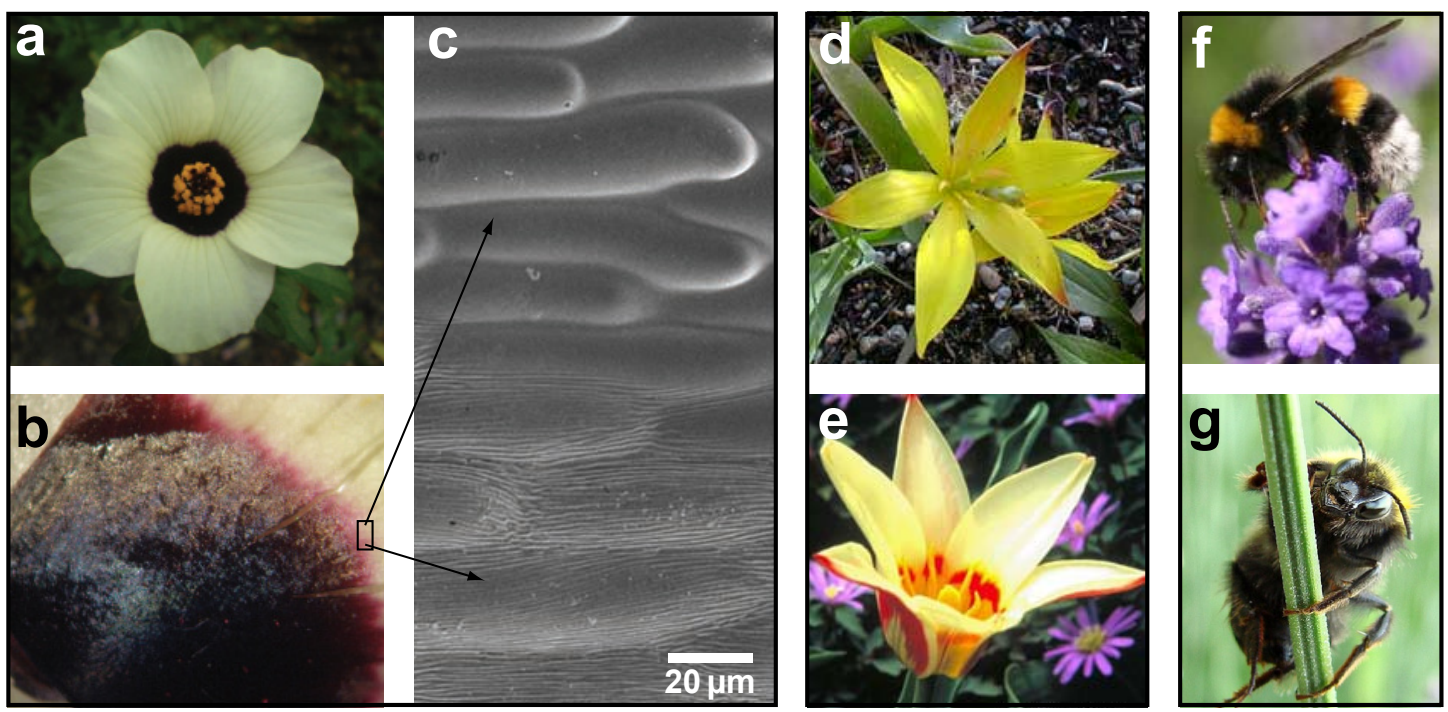

Figure 2.9: a) Hibiscus trionum flower. b) Base of $H$. trionum petal, showing iridescence overlying red pigment. c) SEM of $H$. trionum petal, the upper half of the picture spanning the white (smooth cells) and the lower half spanning the pigmented epidermis, which is heavily striated longitudinally towards the petal base. d) Tulipa kolpakowskiana flower. e) Tulipa kaufmanniana flower (images from www. paghat.com). f), g) Bumblebees, Bombus terrestris (images from http://home.scarlet.be/entomart). 
gratings, surface striations of particular amplitude and periodicity, cause interference, giving rise to an angular colour variation [60]. Although epidermal plant cell shape has been shown to influence the capture of all wavelengths of light by pigments [61, 62], the mechanisms of iridescence have been poorly studied in plants; however, multilayered effects are occasionally observed in leaves [63, 64].

Hibiscus trionum petals are white with a patch of red pigment at the base. This pigmented patch is iridescent, appearing mildly blue, green, and yellow depending on the angle from which it is viewed (Fig. $2.9 \mathrm{a}, \mathrm{b}$ ). Scanning electron microscopy (SEM) shows a sharply defined difference between the surface structure overlying the pigment and the rest of the petal (Fig. $2.9 \mathrm{c}$ ). This iridescence is visible to the human eye; however, in flowers with similar surface structures, such as many species of Tulipa (Table 2.1), the iridescence is only evident to humans when the pigment colour and petal surface structure are separated. When the surface structures of hibiscus and tulip petals were replicated in colourless optical epoxy [65] (Section 3.3.4), iridescent colour was visible independent of pigment (Fig. $2.10 \mathrm{a}, \mathrm{c}$ ).
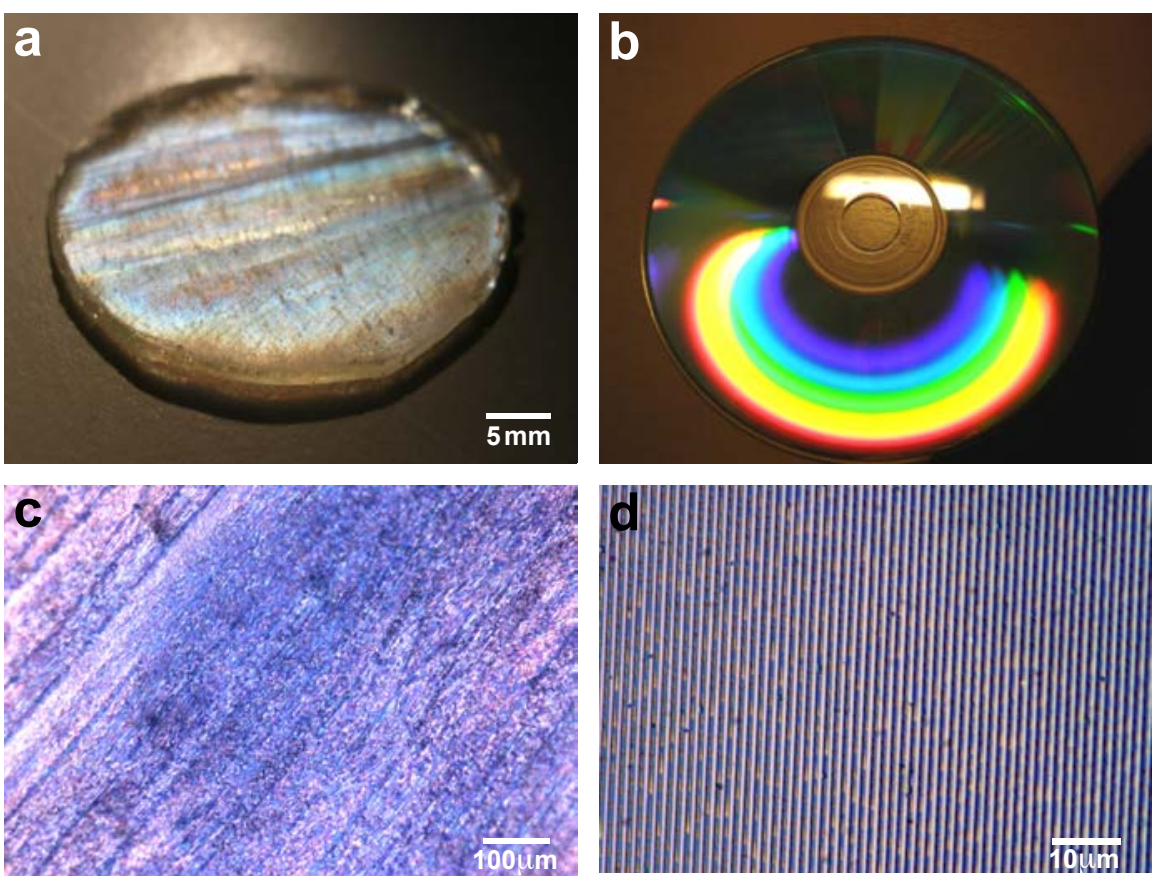

Figure 2.10: Tulip and CD casts. a) Epoxy cast of Tulipa kolpakowskiana. b) Diffraction from a CD-ROM. c) Microscopic image of the striations on the tulip cast surface acquired in reflection. d) Microscope image of the CD structure. 

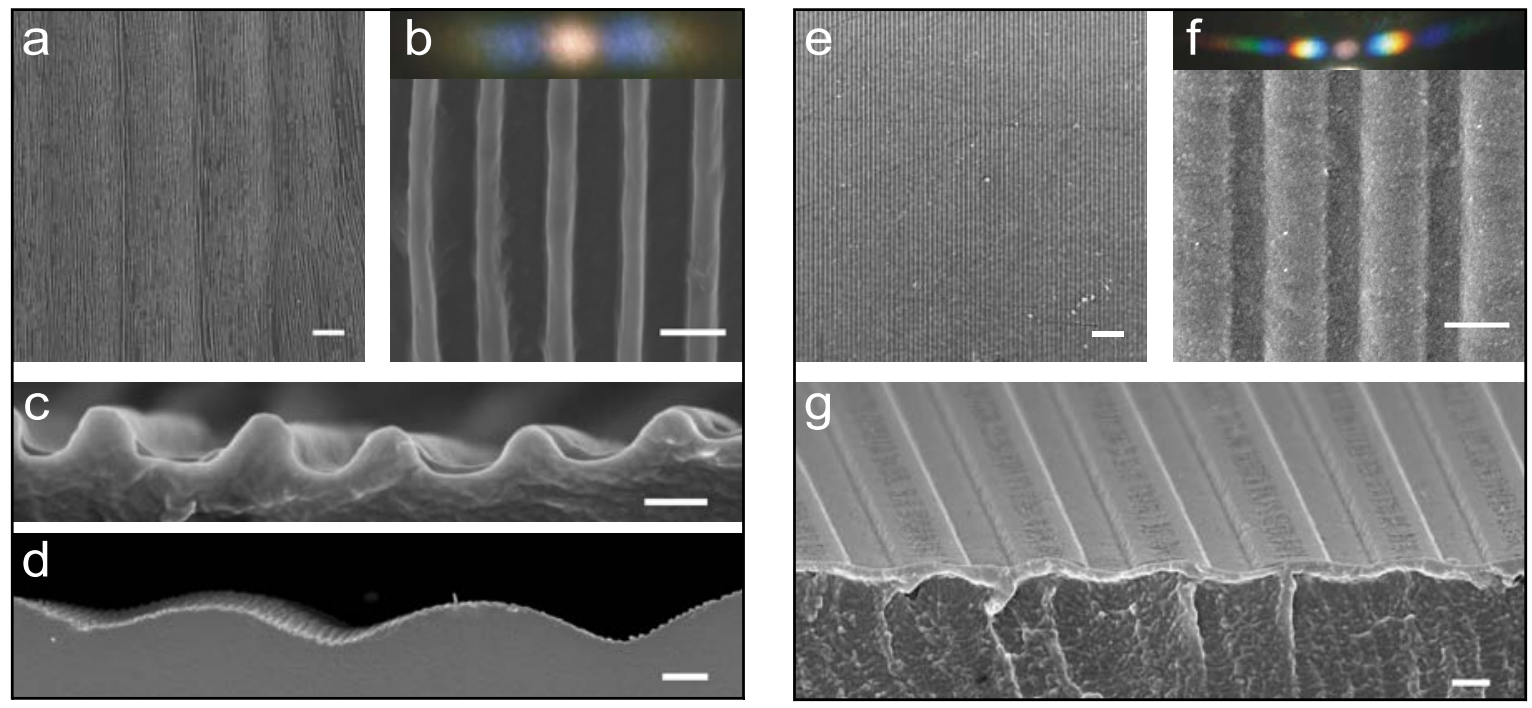

Figure 2.11: SEM images of tulip and CD epoxy casts. a), b) Top view showing striations on the petal surface of Tulipa kolpakowskiana, resembling a line grating with a periodicity of $1.2 \pm 0.3 \mu \mathrm{m}$. c) Side view of the structure from (a), showing the rounded cross-section of the striations. d) The lower magnification image shows undulations with a periodicity of $29 \pm 2 \mu \mathrm{m}$, reflecting the epidermal cells themselves. e), f) Top view of an epoxy cast of a disassembled $C D$, showing a grating periodicity of $1.45 \pm 0.05 \mu \mathrm{m}$. g) Side view of the structure from (f) showing the square cross-section of the CD grating. The insets in (b) and ( $\mathbf{f})$ show the diffraction patterns of the two gratings in transmission. Scale bars: a) $10 \mu \mathrm{m}$, b) $1 \mu \mathrm{m}$, c) $500 \mathrm{~nm}, \mathrm{~d}) 5 \mu \mathrm{m}$, e) $10 \mu \mathrm{m}$, f) $1 \mu \mathrm{m}, \mathrm{g}) 500 \mathrm{~nm}$.

Scanning electron micrographs of these replicas show that long, ordered, cuticular striations overlay the iridescent epidermal cells. These cuticular striations resemble a diffraction grating. The diffraction grating of compact discs (CDs, Fig. 2.10 b, d) has previously been characterised [60], so we used SEM to compare an epoxy cast made of the plastic interior of a disassembled CD with a cast of Tulipa kolpakowskiana (Fig. 2.11).

The tulip cast shows a rounded cross-section of the striations (Fig. 2.11 c, d), as opposed to the square profile of the $\mathrm{CD}$, and a long wavelength undulation with a periodicity of $29 \pm 2 \mu \mathrm{m}$, reflecting the surface of the epidermal cells. 


\section{Optical characterisation}

We investigated the optical properties of tulip casts with light spectroscopy in the 300-to- $880 \mathrm{~nm}$ wavelength range (near-UV to infrared). A collimated light beam was reflected off the cast at an incidence angle $\theta_{\mathrm{I}}=30^{\circ}$. The reflected and scattered light was detected at angles $\theta_{\mathrm{D}}$ varying from $0^{\circ}$ to $90^{\circ}$ in $1^{\circ}$ steps. The angular detection aperture was less than $1^{\circ}$ (Fig. 2.12).

The spectroscope consisted of a fixed light incidence arm, a rotating sample stage and a rotating detector arm with coinciding rotation axes (Fig.2.12 and Fig. 3.11 in Section 3.4.1). White light from a combined deuterium halogen lamp was collimated to form a $2 \mathrm{~mm}$ wide parallel incident beam. A solid angle of detection with a width of less than $1^{\circ}$ was defined by two $2 \mathrm{~mm}$ wide apertures $30 \mathrm{~mm}$ and $130 \mathrm{~mm}$ from the sample and the collected light was coupled by a fibre into an Ocean Optics USB 4000 spectrometer (spectral range: $230 \mathrm{~nm}$ to $880 \mathrm{~nm}$ ).

The spectrally resolved reflectivity was determined for the tulip cast (Fig. 2.13 and

Fig. 2.14 b), a CD cast (Fig. 2.14c) and a planar reference sample (Fig. 2.14a). The

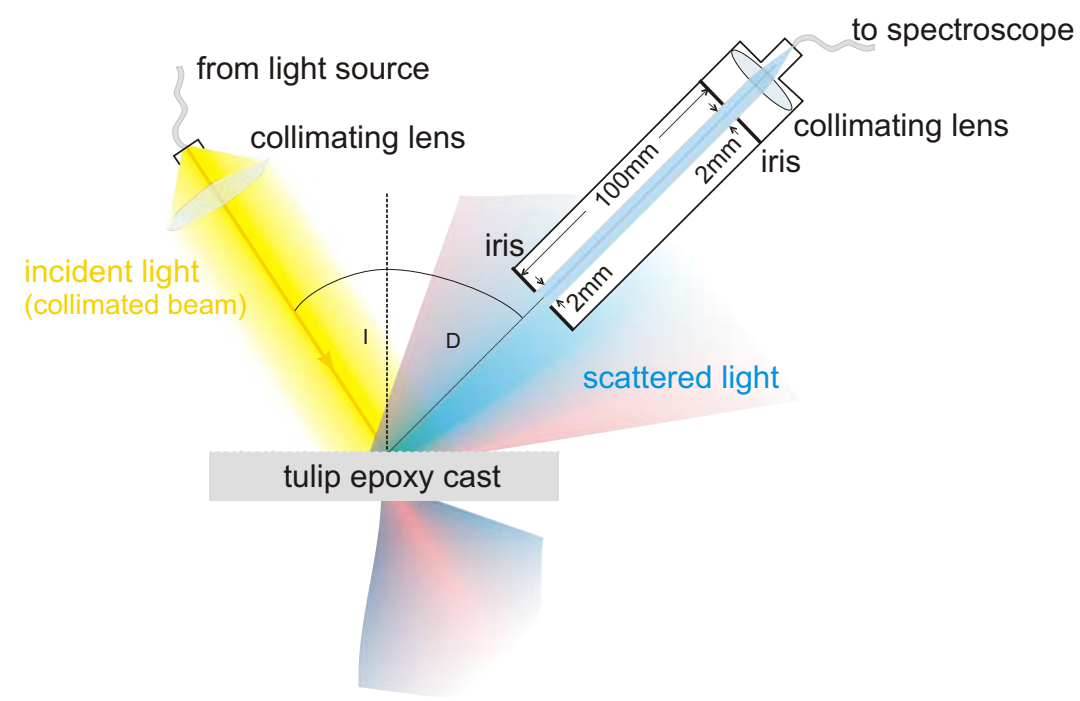

Figure 2.12: Schematic representation of the measurement geometry and the detector set-up. Incident white light was collimated to a beam width of $2 \mathrm{~mm}$. The angular resolution of the detected light was determined by a pair of $2 \mathrm{~mm}$ wide apertures, which were mounted in front of an optical fiber leading the light to a spectrometer. 

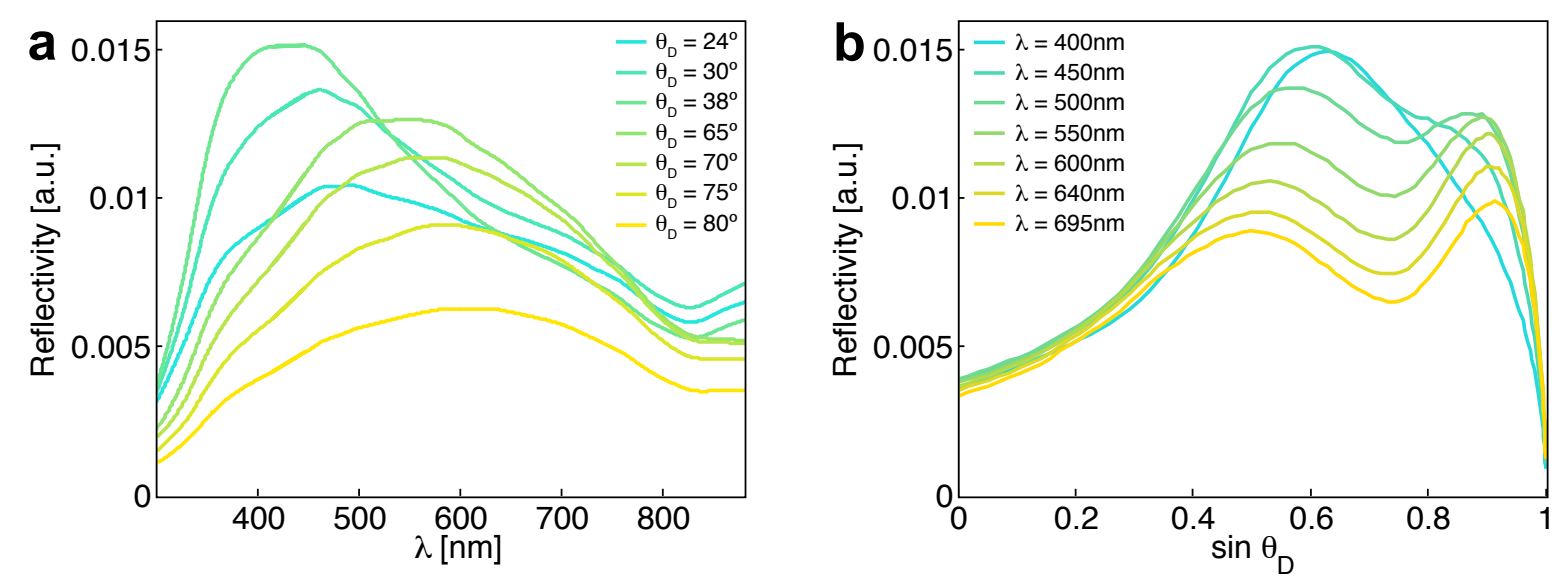

Figure 2.13: Reflectivity of the tulip casts. a) Reflectivity as a function of wavelength $\lambda$. For increasing observation angle $\theta_{\mathrm{D}}>\theta_{\mathrm{I}}$ the reflectivity peak red shifts as expected for diffraction of light of a grating-like structure. b) Reflectivity as a function of observation angle.

reference shows only the specular signal at $\theta_{\mathrm{D}}=30^{\circ}\left(\sin \theta_{\mathrm{D}}=0.5\right)$ for all wavelengths. The CD cast additionally shows first-order diffraction (Fig. 2.14c, diagonal lines) and a weak second-order signal (Fig. $2.14 \mathrm{c}$, bottom left), both of which are quantitatively described by the grating equation $\mathrm{m} \lambda=\mathrm{d}\left(\sin \theta_{\mathrm{D}}-\sin \theta_{\mathrm{I}}\right)$, where $\lambda$ is the wavelength of light, $d$ is the periodicity of the grating $(d=1.45 \mu \mathrm{m})$, and $m$ is the diffraction order [66].

The optical signature of the tulip cast (Fig. 2.13 and Fig. 2.14b) was more complex. The first-order diffraction signal was clearly visible at large angles $\left(\sin \theta_{\mathrm{D}}>0.7\right)$. It is broadened as compared with that of the CD due to the surface undulation caused by the cells shown in Fig. 2.11. The two lines in Fig. 2.14b, which we calculated with the grating equation, delimit the predicted spectral range of first-order diffraction for such a wavy surface. Compared with Fig. 2.14 a,c the specular reflection is broadened and shows an intensity decrease towards long wavelengths. This is a combination of the $\sim 30 \mu \mathrm{m}$ surface undulation and the overall disorder in the pattern of the epidermal cells. Most of the optical intensity is at short wavelengths, coinciding with the high sensitivity of the bee eye in the blue and near-UV ranges [67].

UV signals caused by iridescence are known in animals [56, 68, 69]. Furthermore, bees recognize contrasting patterns in the UV range that occur on flowers [70]. The optical 

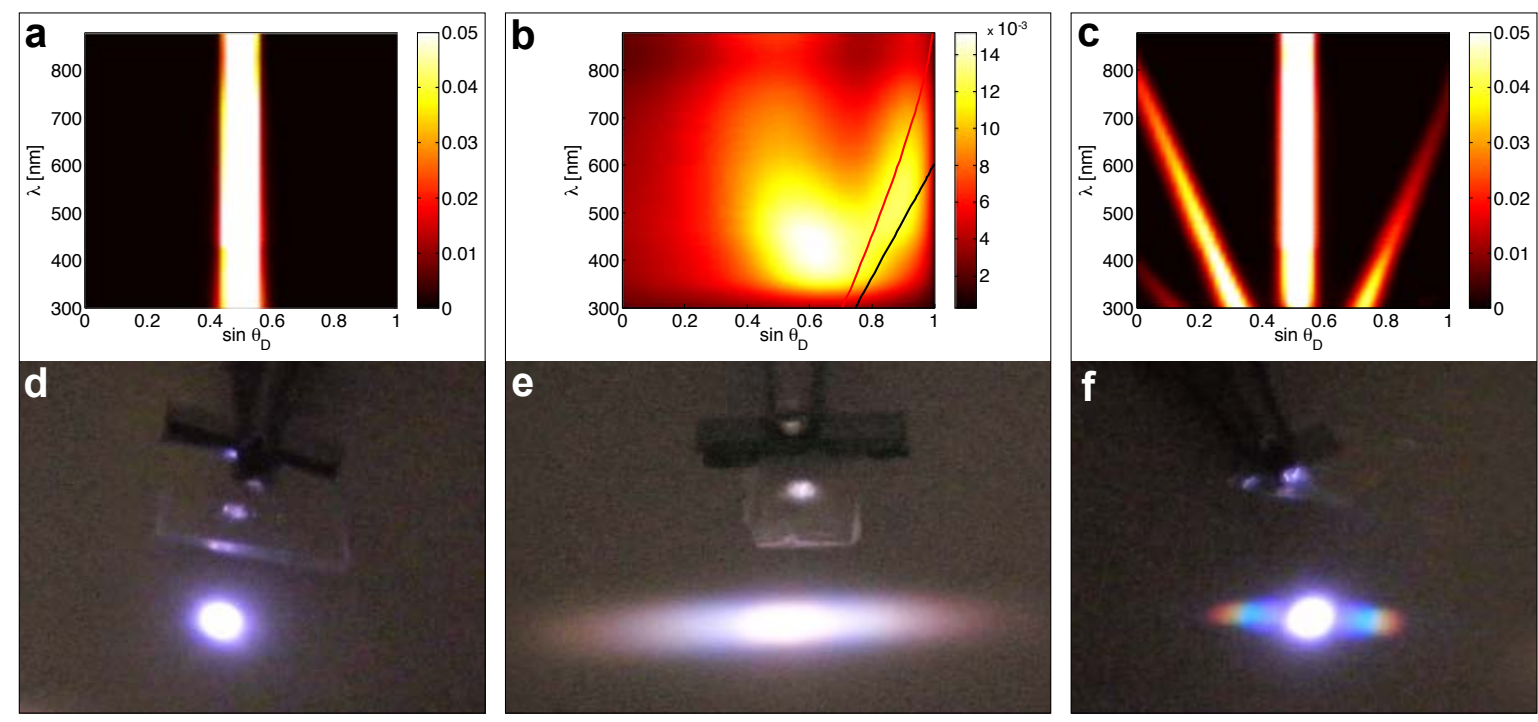

Figure 2.14: Spectrally and angularly resolved reflection of the casts of a tulip, a CD and a flat surface. Reflected intensity of a tulip cast (b) for $\theta_{\mathrm{I}}=30^{\circ}$ as a function of $\sin \theta_{\mathrm{D}}$ and $\lambda$ in comparison to an unstructured surface (a) and a CD cast (c). The central stripe in (a) and (c) is the signature of specular reflection. The two diagonal stripes in (c) are the first-order diffraction of the CD grating, with a weak second-order signal at the bottom left. The tulip cast in (b) shows the clear optical signature of an interference grating with a broadened first-order diffraction for $\sin \theta_{\mathrm{D}}>0.7$. The two lines are the delimiting predictions of the grating equation for a $\sim 30 \mathrm{~mm}$ surface undulation leading to an inclination of the surface normal by $-18^{\circ}$ (red) and $0^{\circ}$ (black). d, e, f, Optical signatures of the three casts in transmission. The flat epoxy (a) and the CD (f) cast show a circular spot in transmission. First-order diffraction is seen for the CD. The long wavelength undulation of the surface of the tulip cast gives rise to the elongated shape of the white transmission spot (e). First order diffraction, although weaker compared to the CD cast is clearly visible.

signature and its angular dependence, because of its particular strength in the UV range, may therefore be more meaningful in terms of insect vision than human vision. Although this optical effect may have its origins in pollinator attraction, striations also occur in many cultivated varieties of tulip (Table 2.1), which may have resulted from additional human selection for the lustre that iridescence lends the flower.

The data analysis of the tulip casts has to take their hierarchical surface structure into account. The sample consisted of two locally regular patterns: grid-like striations with a periodicity of $1.2 \pm 0.3 \mu \mathrm{m}$ (Fig. $2.11 \mathrm{c}$ ) and a long-wavelength undulation with a periodicity of $29 \pm 2 \mu \mathrm{m}$ (Fig. $2.11 \mathrm{~d}$ ). This structure can be approximated with geometrical optics. Figure 2.15 shows a schematic representation of the surface topography 


\begin{tabular}{llll}
\hline \hline Tulip species / variety & Origin & Striations & Order \\
\hline \hline Tulipa acuminata & Iran & Present & Ordered \\
Tulipa biflora & Eastern Mediterranean & Present & Disordered \\
Tulipa edulis & Japan & Present & Disordered \\
Tulipa greigii & Iran, Turkmenistan & Present & Ordered \\
Tulipa hoogiana & Iran, Turkmenistan & Present & Ordered \\
Tulipa humilis & Iran & Present & Ordered \\
Tulipa ingens & Uzbekistan & Present & Ordered \\
Tulipa kaufmanniana & Uzbekistan & Present & Ordered \\
Tulipa kolpakowskiana & Kazakhstan & Present & Ordered \\
Tulipa mauritiana & France & Present & Disordered \\
Tulipa montana & Iran & Present & Ordered \\
Tulipa orphanidea & Greece & Present & Ordered \\
Tulipa pulchella violacea & Turkey & Present & Disordered \\
Tulipa saxatilis & Crete, Turkey & Present & Ordered \\
Tulipa tschimganica & Western Tien Shan & Present & Ordered \\
Tulipa undulatifolia & Turkey & Present & Ordered \\
'Ad rem' & Garden & Present & Ordered \\
'Curly Sue' & Garden & Present & Ordered \\
'Iles de France' & Garden & Present & Ordered \\
'Orange Monarch' & Garden & Present & Ordered \\
'Queen of the Night' & Garden & Present & Ordered \\
'Red Revival' & Garden & Present & Ordered \\
\hline \hline
\end{tabular}

Table 2.1: Tulip species and garden varieties analysed for presence or absence of ordered striations. Study done by Dr. Heather Whitney.

on the $30 \mu \mathrm{m}$ length scale. Because of the surface undulation, the surface normal varies as a function of the lateral position, with a maximal inclination with respect to the averaged surface normal of $\theta_{\mathrm{u}}= \pm\left(18^{\circ} \pm 2^{\circ}\right)$, corresponding to the points of inflection of the undulation. This variation of the surface normal has to be taken into account in the grating equation: $\mathrm{m} \lambda=\mathrm{d}\left[\sin \left(\theta_{\mathrm{D}}+\theta_{\mathrm{u}}\right)-\sin \left(\theta_{\mathrm{I}}+\theta_{\mathrm{u}}\right)\right]$. As a consequence, the diffraction peaks are smeared out over an angular range. The lines in Fig. 2.14 b correspond to $\theta_{\mathrm{u}}=-18^{\circ}(\mathrm{red})$ and $\theta_{\mathrm{u}}=0^{\circ}$ (black). Positive values of $\theta_{\mathrm{u}}$ contribute much less to the diffraction pattern compared to $\theta_{\mathrm{u}} \leq 0$, as illustrated in Fig. 2.15. Regions with surface normals that are inclined towards the incident beam contribute much more to the diffraction pattern as compared to 'shadow' regions, where the surface normal is tilted away from the incident beam. The surface undulation also leads to the angular variation of the specular reflection, which generates the broad intensity distribution 


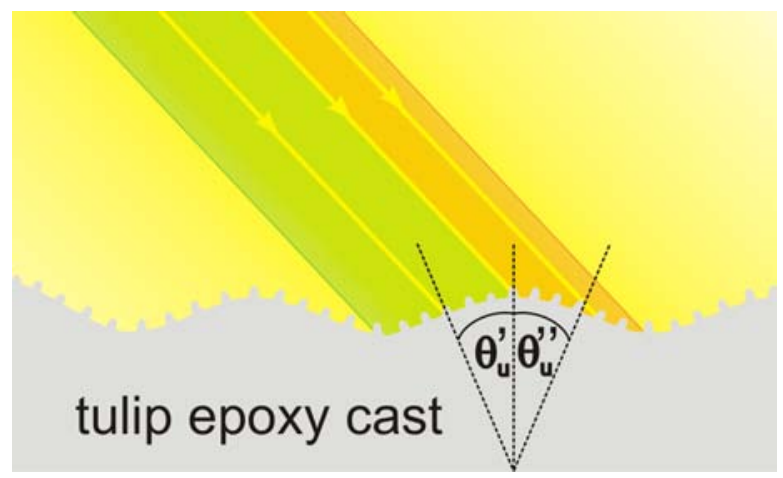

Figure 2.15: Local geometrical optics of an undulating surface. The nominal angle of incidence with respect to the surface normal $\theta_{\mathrm{I}}$ applies locally only at the undulation maxima and minima. At all other locations the surface normal (dashed lines) are inclined with respect to the nominal surface normal. This results in an angular offset $\theta_{\mathrm{u}}$, which is a function of the lateral surface position. The maximal deviations from the nominal surface normal, $\theta_{\mathrm{u}}^{\prime}, \theta_{\mathrm{u}}^{\prime \prime}$, occur at the inflection points of the undulation. From Fig. $2.11 \mathrm{~d}$, a maximum value of $\theta_{\mathrm{u}}^{\prime \prime}=\theta_{\mathrm{u}}^{\prime}=18^{\circ} \pm 2^{\circ}$ was determined. Note that surface regions with a surface normal inclined towards the incident light beam (green) are relatively stronger illuminated compared to regions where the surface normal is tilted away from the direction of the incident light (orange).

around $\sin \theta_{\mathrm{D}} \approx 0.6$ and contributes to the finite background intensity in the entire range of $\theta_{\mathrm{D}}$. The minus first order diffraction is not discernible in Fig. 2.14 b.

Animals use iridescence for species recognition and mate selection [48, 55-57]. Iridescence is under selective pressure in some species - for example, arising from intraspecific competition between male butterflies in their attractiveness to females [57]. Floral iridescence, in contrast, is presumably a signal to pollinating animals.

\section{Iridescence, a cue for pollinators}

To test whether iridescence, as displayed by Hibiscus trionum, is distinguished by pollinators, we measured the variation in hue shown by the iridescent patch, with spectroscopy both across the striations (measuring maximum iridescence) and along the striations (measuring minimum iridescence). The colour loci of these two measurements were calculated in a bee hexagon colour space ${ }^{4}$ [71] (Fig. 2.16 a). This indicated that

\footnotetext{
${ }^{4} \mathrm{~A}$ representation of colour perception designed using information about receptor sensitivity and colour-opponent coding, so that distances between points generated by two objects indicate the degree to which the two are distinguishable.
} 
bees will perceive a change in flower colour from different angles. We observed that the difference in colour between the two measurements, when calculated as the Euclidean distance between colour loci, was 0.217 . As a colour distance of 0.15 is distinguishable by bees with above $90 \%$ accuracy and even a distance of 0.05 can be distinguishable by trained bees [72], the variation in hue demonstrated by a single Hibiscus trionum flower viewed from different angles is sufficient for ready visual discrimination by bees.

Dr. Heather Whitney tested the ability of flower-naïve bumblebees to discriminate between iridescent and noniridescent disks by using differential conditioning [73]. All bee experiments described in the following were performed by Dr. Whitney and are displayed here for completeness in order to put the optical study of diffraction structures on flower in the appropriate context. Iridescent disks of epoxy resin were generated by casting in a dental wax impression of a $3 \mathrm{~cm}$ diameter disk of the dissected plastic diffraction gratings removed from within a CD. Non-iridescent disks were cast in a wax impression of smooth molded plastic. $50 \mu \mathrm{g}$ of pigment (ultramarine blue, chrome yellow, manganese violet or quinacridone red) were added to every $10 \mathrm{~g}$ of epoxy. Three non-iridescent and three iridescent of each of the yellow, blue and violet disks were placed on Sterilin tubes in a flight arena, to which a flower-naïve bumblebee colony was connected by a gated tube. Small cups glued to the iridescent disks were filled with $15 \mu \mathrm{l} 30 \%$ sucrose solution, cups on the non-iridescent disks with $15 \mu \mathrm{l} 0.12 \%$ quinine hemisulphate salt solution. Bees' olfactory system can not discriminate between sucrose and quinine solutions, forcing them to feed from the solution in order to access its taste [73]. The artificial flowers were refilled regularly, re-arranged randomly and cleaned with ethanol between foraging bouts.

Illumination for the behavioural experiments was provided by six Sylvania Activa 172 Professional $36 \mathrm{~W}$ fluorescent tubes (Germany) that were modified with Phillips high frequency ballasts to have a flicker frequency greater than $1200 \mathrm{~Hz}$ on a 12 hour light / dark regime.

Bees were trained that iridescent disks containing yellow, blue, or violet pigment offered a sucrose reward, whereas identically pigmented non-iridescent disks offered a 

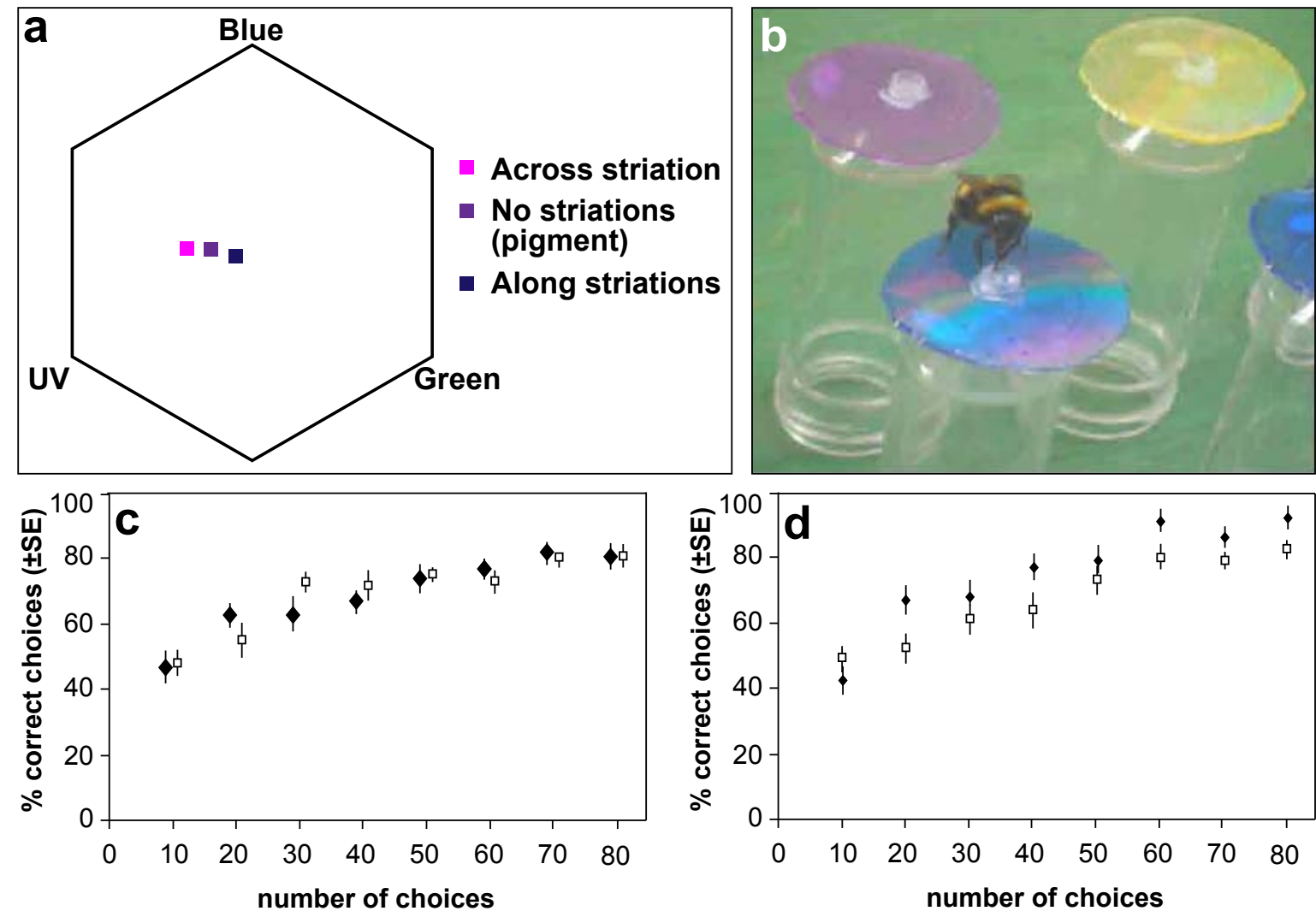

Figure 2.16: Iridescence, a cue for pollinators. a) Loci of Hibiscus trionum in the bee color hexagon. b) Bee learning to associate iridescent epoxy flowers with a reward. c) Two learning curves, each of 10 bees (with standard deviation), choosing between rewarding iridescent flowers and nonrewarding, noniridescent flowers. Filled diamonds indicate bees that were offered casts of CDs; clear squares indicate bees that were offered casts of tulip petals. d) Learning curves of 10 bees, choosing between rewarding iridescent purple flowers and non-rewarding non-iridescent purple flowers when all flowers were covered with a sheet of depolarising mylar (filled diamonds) or UV-blocking polycarbonate (empty squares). Lack of polarisation or UV signal does not prevent the bees from distinguishing the iridescent from the noniridescent surfaces. All data was acquired by Dr. Heather Whitney.

bitter quinine hemisulphate salt solution [73] (Fig. 2.16 b). After 80 visits, bees visited iridescent disks more frequently than after their immediate introduction to the arena (first 10 visits $=4.7 \pm 0.5$; last 10 visits $=8.1 \pm 0.4$; see Fig. $2.16 \mathrm{c}$ ). Preference for iridescence did not differ according to pigment colour. For each bee, these disks were then removed and replaced with five noniridescent and five iridescent red disks. When shown this previously unseen colour, bees continued to visit iridescent flowers with $(83.4 \pm 3.4) \%$ accuracy during their first foraging bout, demonstrating their ability to use iridescence as a cue to distinguish between rewarding and nonrewarding substrates 
irrespective of pigment-based reflectance. This suggests that although pollinators are typically thought to identify rewarding flowers by their pigment-based reflectance, in our studies they were able to discriminate between the disks having a weak superposed angular-dependent colour signal arising from grating interference.

The polarisation of light reflected by iridescent colours on female Heliconius butterflies is recognised by males, but the changing colours are not [48]. To assess if the visual cue used by the bumblebees was independent of a polarisation effect, we repeated the discrimination experiment with only violet pigment disks and depolarising Mylar over each disk. This removed polarisation signals but left the colour intact [48]. Bees were again more likely to visit iridescent flowers as time progressed (first 10 visits $=4.3 \pm 0.50$; last 10 visits $=9.2 \pm 0.34$ ). We repeated the experiment again with a polycarbonate filter opaque to wavelengths below $400 \mathrm{~nm}$ [74] blocking any UV signal, to ensure that the UV component of the diffraction grating was not acting as a specific cue. After 80 visits, bees visited iridescent disks more frequently than after their immediate introduction to the arena (first 10 visits $=4.9 \pm 0.41$; last 10 visits $=8.2 \pm 0.25$ ). Learning curves are shown in Figure $2.16 \mathrm{~d}$ ). We conclude that iridescence generated by diffraction gratings can be used as a pollination cue by bumblebees independent of underlying pigment, UV signals, or polarisation effects.

To confirm that bees could discriminate the less regular iridescence of a real flower, yellow-pigmented epoxy casts were made from Tulipa kolpakowskiana petals. Casts with floral iridescence were taken from the adaxial petal surface, which has striations. Casts without iridescence were taken from the abaxial petal surface, which lacks striations. Overall epidermal cell size and shape was similar on both surfaces. Our results show that bees were able to use the floral iridescence as accurately and effectively as they used the CD iridescence as a pollination cue. After 80 visits, bees visited iridescent disks more frequently than after their immediate introduction to the arena (first 10 visits $=4.8 \pm 3.89$, last 10 visits $=8.1 \pm 3.14$, see Fig. $2.16 \mathrm{c}$ ).

Over $50 \%$ of angiosperm species produce a striated cuticle over their petals [75], and although the degree to which such striations are ordered will strongly influence their 


\begin{tabular}{ll}
\hline \hline Family & Example species \\
\hline \hline Malvaceae & Hibiscus trionum \\
Liliaceae & Tulipa sp \\
Asteraceae & Gazania krebsiana \\
Fabiaceae & Ulex europaeus \\
Loasaceae & Mentzelia lindleyi \\
Ranunculaceae & Adonis aestivalis \\
Onagraceae & Oenothera biennis \\
Solanaceae & Nolana paradoxa \\
Iridaceae & Ixia viridiflora $(\mathrm{S} 3)$ \\
Paeoniaceae & Paeonia lactiflora \\
\hline \hline
\end{tabular}

Table 2.2: Angiosperm families and species in which iridescence generated by diffraction gratings has been observed. All species directly observed, except (S3). Study done by Dr. Heather Whitney.

visual effect, it is nonetheless probable that many flowers produce iridescence. We have so far identified ten angiosperm families containing species with petal iridescence generated by diffraction gratings (table 2.2). Such striations may also influence pollinators through their tactile effects [76]. As demonstrated by Hibiscus trionum, structures causing iridescence may occur in an overlying pattern to those caused by pigment colour. This floral patterning is known to be important in pollinator attraction [70, 77]. It has previously been shown in both birds and butterflies that structural colour can enhance pigment colour either by an additive or a contrast effect [68, 78-80]. This interplay of structure and pigment may also add to the diversity of pollination cues utilised by the flowers of many angiosperm species.

\subsubsection{Near-UV reflectivity enhancement by grating-like stria- tions}

Striations on the investigated flower petals can cause diffraction and ultimately dynamically varying colours, nevertheless they may first have appeared in the evolution of plants to serve a different purpose. Common plant pollinators, including many bee species, rely on highly sensitive vision in the ultraviolet spectral range (UV) [69, 70]. 

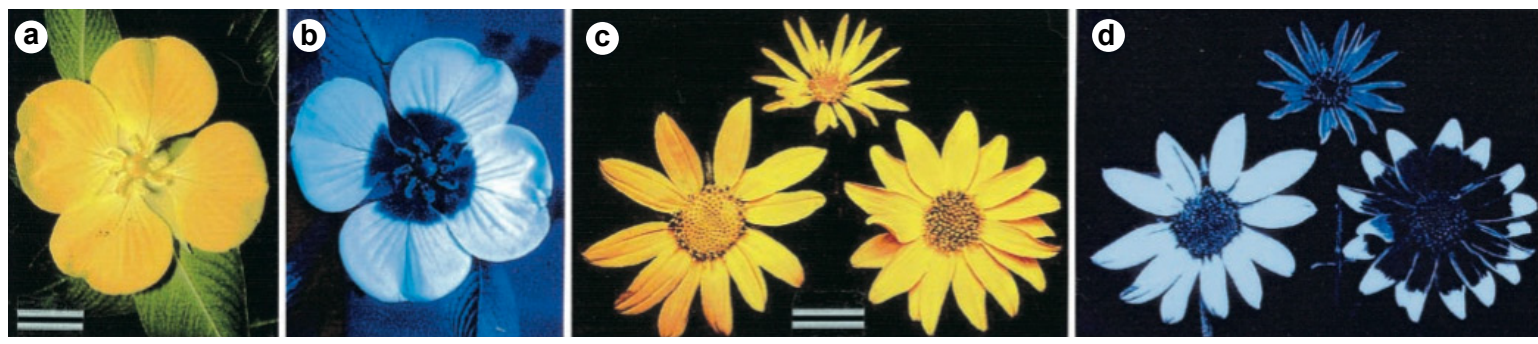

Figure 2.17: Flower petals in visible and UV light. UV images are shifted into the blue. a), b) Ludwigia peruviana. c), d) Chrysopsis villosa (left), Helianthella quinqueneris (top), and Viguiera multiflora (right). Scale bars $1 \mathrm{~cm}$. All images are taken from [81].

The capacity of signalling in the UV-range seems to create an evolutionary advantage for flowering plants, increasing their ability to attract pollinators with UV-sensitive vision. Indeed, many flowers have acquired the ability to manipulate UV-light present in the solar spectrum. These plants usually employ UV-absorbing pigmentation to create UV-demarcations on their flower petals [81]. This UV-pigmentation, often concentrated in the centre of the flower, results for example in dark 'bulls eye'-like patches (Fig. 2.17). However, pigments with the capacity to strongly emit or scatter UV-light are not readily available in nature. The high reflectivity of striated petal surfaces in the far-blue to near-UV spectral range (Fig. 2.13) suggests that these grating-like structures have developed to allow plants to display intricate UV-patterns not only through the use of UV-absorbing pigments but also by reflection and redirection of UV-light present in the solar spectrum.

With this hypothesis in mind, reflection from the striated tulip flower surfaces (Fig. 2.11) is currently being modelled using the FDTD simulation package MEEP (Chapter 1.6.2).

Analysis of the reflection from Mentzelia lindleyi flower petals supports the UV-hypothesis. This work was done in collaboration with Timo Stein, summer student in the Thin Films and Interfaces group in 2008. The annual wildflower Mentzelia lindleyi, commonly known as "evening star" or "blazing star", blossoms in the coastal ranges of California and in Arizona, with each plant displaying many small, five-petaled, brightyellow flowers (Fig 2.18 a). Striations, aligned along the petal axis, cover the flower petal surface. These striations are quite regularly spaced by $(1.2 \pm 0.3) \mu \mathrm{m}$ with feature 

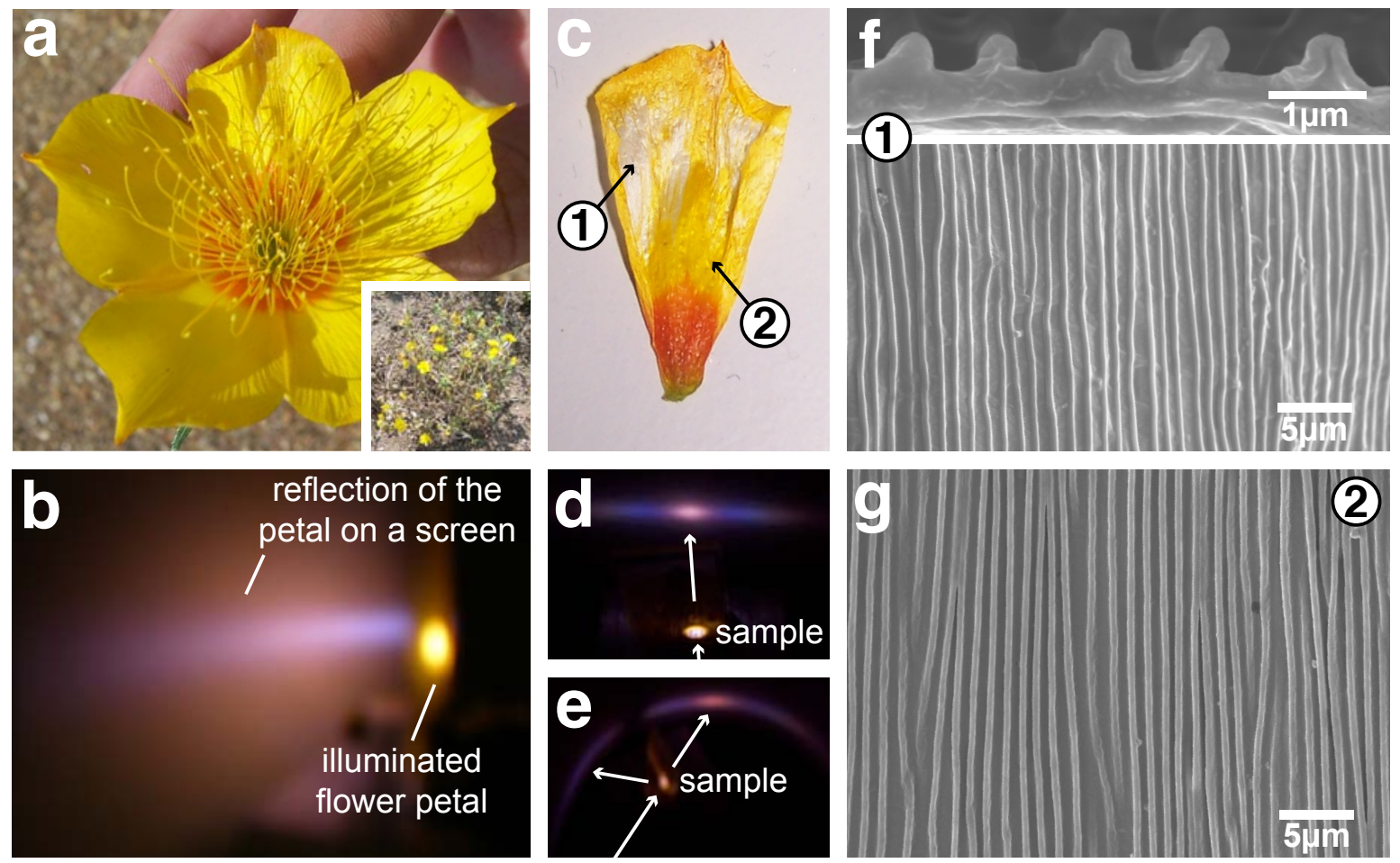

Figure 2.18: Mentzelia lindleyi. a) A flower of Mentzelia lindleyi. The inset shows the whole plant. b) The reflection of a flower petal, captured on a white screen. The petal is illuminated with a collimated white light beam at an incidence angle of $\theta_{\mathrm{I}}=30^{\circ} \mathrm{C}$. The striations on the flower petal are oriented perpendicular to the plan of incidence. Two features are distinguishable in the reflected light, a less intense oval yellow scatter and a distinct blue-white stripe in the plane of incidence. c) A flower petal, after having been locally exposed to strong UV-light for several hours. The yellow pigmentation in the exposed regions bleaches, leaving these areas semi-transparent (1). d) Transmission of light through a bleached part of the flower petal, at normal light incidence, resulting in a diffraction pattern on a flat screen behind the sample. The white arrows indicate the light path. e) Reflection and transmission of light incident on a bleached area of the petal, captured on a cylindrically curved screen.f) Scanning electron micrograph of a bleached flower petal area (1) in cross-section and in top view. g) SEM image of an unbleached, yellow petal area in top view. Pictures in (a), (c), (d), and (e) were acquired by Timo Stein.

heights of $\sim 0.4 \mu \mathrm{m}$. When a collimated white light beam impinges on the petal surface, aligned with its striations perpendicular to the plane of light incidence, the projection of the reflected light onto a white screen contains two distinct features: a yellow oval spot that is superposed by a brighter blue-white stripe, lying in the plane of incidence (Fig $2.18 \mathrm{~b}$ ). The stripe rotates on the screen as the petal is rotated around its surface normal, suggesting that this particular optical signature results from diffraction caused 
by the striations. The underlying yellow spot is presumably due to the scattering from the flower pigments.

Local exposure of a flower petal to UV-light for several hours causes degradation of the pigmentation, rendering the exposed areas semi-transparent (Fig 2.18c, (1) exposed, (2) not exposed). The surface striations however, remain untouched. This was verified by comparing the exposed and non-exposed areas in a scanning electron microscope (compare Fig $2.18 \mathrm{f}$ and g). After UV-treatment, a diffraction pattern can be seen in transmission for normal illumination of the semi-transparent areas on the petal (Fig 2.18d). Reflection and transmission from these areas are visualised on a curved screen for a light incidence angle of $30^{\circ}$ (Fig. $2.18 \mathrm{e}$ ). While the yellow scatter has now disappeared a blue stripe, comparable to the one obtained for the intact flower surface, persists in reflection, strongly suggesting that this feature results from diffraction caused by the surface striations.

Spectroscopic investigations of a petal's scattering behaviour were performed by shining light onto the flower surface at a fixed incidence angle, $\theta_{i} \approx 30^{\circ}$. Spectra were acquired at detection angles, $\theta_{\mathrm{D}}$ between $0^{\circ}$ and $70^{\circ}$. Measurements were taken in three different geometries that are represented together with the obtained results in Figure 2.19.

In the first geometry, the striations were oriented perpendicularly to the plane of incidence, to capture the angular spectral signature of the blue-white stripe in reflection (Fig. $2.19 \mathrm{a}$ ). For the second measurement, the flower petal was tilted out of the original plane of incidence, to avoid detection of the blue-white stripe, while capturing the light in the yellow scattering cone (Fig. 2.19 b). In the last measurement the striations at the petal surface were aligned in the plane of incidence (Fig. $2.19 \mathrm{c}$ ).

Colour-coded reflectivity spectra are shown as a function of wavelength $\lambda$ and the sine of the detection angle $\theta_{\mathrm{D}}$ for all geometries in the second row of Figure 2.19. Blue, red and green lines mark the angles for which reflection spectra as a function of wavelength are displayed in the third row.

The broad reflectivity plateau above $520 \mathrm{~nm}$ results from pigment scattering. The absolute reflectivity of $35-40 \%$ in this range corresponds well to values reported for 

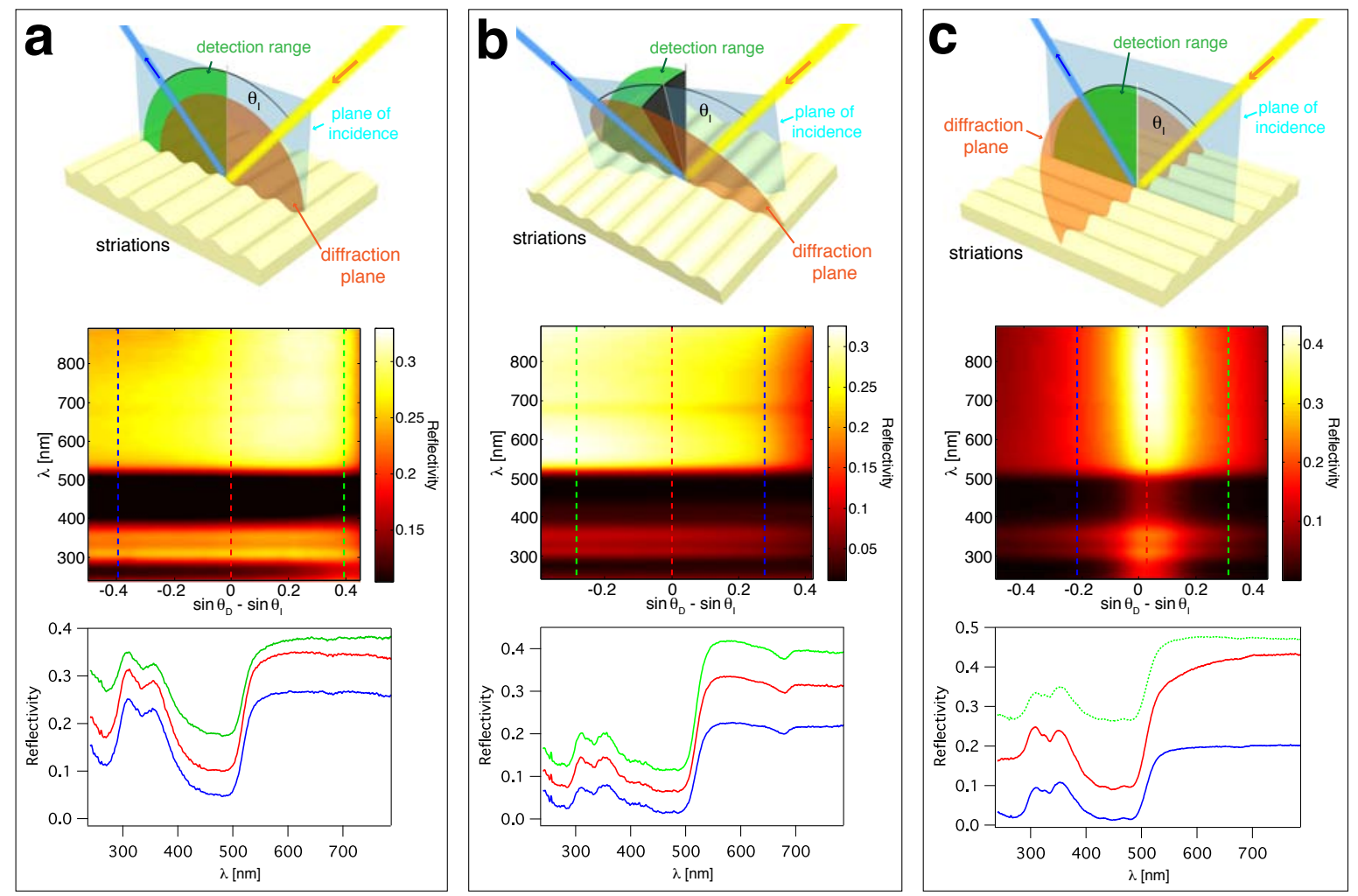

Figure 2.19: Spectroscopic investigation of Mentzelia lindleyi. Schematic of the measurement geometries and resulting reflectivity data for detection in the diffraction plane of the striations (a), for detection with the sample normal tilted out of the detection plane (b) and for detection perpendicular to the diffraction plane (c). In each schematic, the plane of incidence is shown in light blue, the detection plane in green and the diffraction plane that depends on the orientation of the striations is shown in red. Color coded reflectivity spectra as a function of detection angle and wavelength are shown in the second row. Dashed blue, red, and green lines mark the detection angles for which reflectivity spectra as a function of wavelength are displayed in the third row. For clarity, in (a) and (b) the red and green spectra are offset from the blue plot by 0.05 and 0.1 . In (c) only the green line is offset by 0.25 .

the average reflectivity of flowers [82]. The double peak structure in the spectral range of $300-400 \mathrm{~nm}$ is unlikely to result from pigment scattering. This feature weakens strongly if the sample is tilted slightly out of the incidence and detection plane of the spectroscope, while the plateau above $520 \mathrm{~nm}$ remains. (Fig. $2.19 \mathrm{~b}$ ). Similarly, the double peak structure is less pronounced if the striations of the petals are aligned in the plane of incidence (Fig. $2.19 \mathrm{c}$ ), which positions the detection plane perpendicular to the diffraction plane of the striations. In this geometry the reflectivity drops over the whole spectral range for detection angles smaller or larger than $\theta_{\mathrm{D}} \approx 30^{\circ}$, which 
corresponds to the direction of specular reflection. This observation suggests that the striations presumably also cause a homogeneous dispersion of reflected light in the diffraction plane.

The results do not suggest that the reflection characteristics of an undamaged pigmented flower petal simply result from a superposition of the pigment-based scattering and the diffraction / scattering induced by the striations. It is likely that the interaction between surface structure and pigmentation is somewhat more complex. To understand the discrepancies between the spectral signature of pigment deprived petals and undamaged petal surfaces, further experiments should be conducted. The pigment needs to be separated from the flower and analysed spectroscopically, while the epidermis of the flower supporting the striations needs to be detached from the petal and investigated on its own.

\subsection{Conclusion}

Despite their different evolutionary development, all insect species, discussed in the first part of this chapter, show important similarities in their approach to the creation of colour. Multilayer structures, often made from transparent media are an essential ingredient. Nevertheless, multilayer structures do not, on their own, account for the optical appearance of these species. Well-positioned pigment pockets, periodic surface corrugations or well defined inclination of adjacent scales, are employed to complement the effect of multilayers, leading to unique colour patterns. Grating-like micro-striations shape the reflection properties of flower petal surfaces, by diffraction and directional scattering, as seen in the second part of this chapter.

Organisms in fauna and flora show us possible routes to create and tailor photonic micro- and nano-structures that give rise to beneficial combinations of different optical effects, satisfying the individual requirements.

An artificial optical surface structure that displays stunning, bright and vivid colours has to be composed of periodic elements on several length scales, combined with ab- 
sorption agents in appropriate spatial arrangement within or under the structure. The precise configuration depends strongly on the purpose which the optical structure is supposed to satisfy. The inclusion of air as low refractive index material allows for enhanced optical performance, leading to brighter, more intense, vivid colours. Surface diffractive elements can help to disperse light reflected from pigmentation or multilayered structures to influence the directions of maximum reflection. The lessons learnt from nature concerning the design of photonic structures with complex tailored optical signature, for instance the arrangement of cuticle-air multilayers into concave shapes, should help to design and realise photonic structures with similarly impressive or even enhanced optical properties. 


\section{References}

[1] Butcher, G. \& Rohwer, S. The evolution of conspicuous and distinctive coloration for communication in birds. Curr. Ornithol. 6, 51-108 (1989).

[2] Baker, R. \& Parker, G. The evolution of bird coloration. Philos. T. Roy. Soc. B 287, 63-130 (1979).

[3] Guilford, T. The evolution of conspicuous coloration. Am. Nat. 131, 7-21 (1988).

[4] Prudic, K., Skemp, A. \& Papaj, D. Aposematic coloration, luminance contrast, and the benefits of conspicuousness. Behav. Ecol. 18, 41 (2007).

[5] Kevan, P. \& Baker, H. Insects as flower visitors and pollinators. Annu. Rev. Entomol. 28, 407-453 (1983).

[6] Vigneron, J. P. et al. Optical structure and function of the white filamentary hair covering the edelweiss bracts. Phys. Rev. E 71, 8 (2005).

[7] Lord Rayleigh. The iridescent colours of birds and insects. Proc. Roy. Soc. A 128, 624-641 (1930).

[8] Vukusic, P. \& Sambles, J. R. Photonic structures in biology. Nature 424, 852-855 (2003).

[9] Vukusic, P. Structural colour in Lepidoptera. Curr. Biol. 16, R621-R623 (2006).

[10] Srinivasarao, M. Nano-optics in the biological world: Beetles, butterflies, birds, and moths. Chem. Rev. 99, 1935-1962 (1999).

[11] Doucet, S. \& Meadows, M. Iridescence: A functional perspective. J. Roy. Soc. Interface 6, S115-S132 (2009).

[12] Meadows, M. et al. Iridescence: Views from many angles. J. Roy. Soc. Interface 6, S107-113 (2009). 
[13] Land, M. The physics and biology of animal reflectors. Prog. Biophys. Mol. Bio. 24, 75 (1972).

[14] Ghiradella, H. Development of ultraviolet-reflecting butterfly scales: How to make an interference filter. J. Morphol. 142, 395-409 (1974).

[15] Vukusic, P., Sambles, J., Lawrence, C. \& Wootton, R. Quantified interference and diffraction in single Morpho butterfly scales. Proc. Roy Soc. B - Biol. Sci. 266, 1403 (1999).

[16] Prum, R., Torres, R., Williamson, S. \& Dyck, J. Coherent light scattering by blue feather barbs. Nature 396, 28-29 (1998).

[17] Berthier, S. Iridescences: The physical colors of insects (Springer, 2007).

[18] Kinoshita, S. \& Yoshioka, S. Structural colors in nature: The role of regularity and irregularity in the structure. ChemPhysChem 6, 1442-1459 (2005).

[19] Vukusic, P., Sambles, J. \& Lawrence, C. Structural colour: Colour mixing in wing scales of a butterfly. Nature 404, 457 (2000).

[20] Vukusic, P., Hallam, B. \& Noyes, J. Brilliant whiteness in ultrathin beetle scales. Science 315, 348 (2007).

[21] Vukusic, P., Sambles, J. \& Lawrence, C. Structurally assisted blackness in butterfly scales. Proc. Roy Soc. B - Biol. Sci. 271, S237-S239 (2004).

[22] Sharma, V., Crne, M., Park, J. \& Srinivasarao, M. Structural origin of circularly polarized iridescence in jeweled beetles. Science 325, 449 (2009).

[23] Berthier, S., Boulenguez, J. \& Bálint, Z. Multiscaled polarization effects in Suneve coronata (Lepidoptera) and other insects: Application to anti-counterfeiting of banknotes. Appl. Phys. A-Mater. 86, 123-130 (2007).

[24] Biro, L. P. et al. Bioinspired artificial photonic nanoarchitecture using the elytron of the beetle Trigonophorus rothschildi varians as a 'blueprint'. J. Roy. Soc. Interface (2009). 
[25] Michielsen, K. \& Stavenga, D. Gyroid cuticular structures in butterfly wing scales: Biological photonic crystals. J. Roy. Soc. Interface 5, 85 (2008).

[26] Michielsen, K., Raedt, H. D. \& Stavenga, D. G. Reflectivity of the gyroid biophotonic crystals in the ventral wing scales of the green hairstreak butterfly, Callophrys rubi. J. Roy. Soc. Interface 7, 765-771 (2010).

[27] Whitney, H., Kolle, M., Andrew, P., Chittka, L., Steiner, U. \& Glover, B. J. Floral iridescence, produced by diffractive optics, acts as a cue for animal pollinators. Science 323, 130-133 (2009).

[28] Whitney, H., Kolle, M., Alvarez-Fernandez, R., Steiner, U. \& Glover, B. Contributions of iridescence to floral patterning. Communicative $\&$ Integrative Biology 2, 230-232 (2009).

[29] Thomas, K. R., Kolle, M., Whitney, H. M., Glover, B. J. \& Steiner, U. Function of blue iridescence in tropical understorey plants. submitted (2010).

[30] Prum, R. \& Torres, R. Structural colouration of mammalian skin: Convergent evolution of coherently scattering dermal collagen arrays. J. Exp. Biol. 207, 2157 (2004).

[31] Vukusic, P. Advanced photonic systems on the wing-scales of Lepidoptera. In Gorb, S. N. (ed.) Functional surfaces in biology: Little structures with big effects. (Springer, 2009).

[32] Prum, R. O. Structural colouration of avian skin: Convergent evolution of coherently scattering dermal collagen arrays. J. Exp. Biol. 206, 2409-2429 (2003).

[33] Parker, A. \& Martini, N. Structural colour in animals - simple to complex optics. Opt. Laser Technol. 38, 315-322 (2006).

[34] Mcphedran, R. Structural colours through photonic crystals. Physica B 338, 182-185 (2003).

[35] Parker, A. R. A geological history of reflecting optics. J. Roy. Soc. Interface 2, $1-17(2005)$. 
[36] Parker, A. A vision for natural photonics. Philos. T. Roy. Soc. A 362, 2709-2720 (2004).

[37] Prum, R., Quinn, T. \& Torres, R. Anatomically diverse butterfly scales all produce structural colours by coherent scattering. J. Exp. Biol. 209, 748 (2006).

[38] Seago, A., Brady, P., Vigneron, J. \& Schultz, T. Gold bugs and beyond: A review of iridescence and structural colour mechanisms in beetles (Coleoptera). J. Roy. Soc. Interface 6, S165 (2009).

[39] Kinoshita, S., Yoshioka, S. \& Miyazaki, J. Physics of structural colors. Rep. Prog. Phys. 71, 76401-76500 (2008).

[40] Vukusic, P., Sambles, J. \& Ghiradella, H. Optical classification of microstructure in butterfly wing scales. Photonics Science News 6, 61-66 (2000).

[41] Ghiradella, H. Structure of iridescent Lepidopteran scales: Variations on several themes. Ann. Entomol. Soc. Am. 77, 637-645 (1984).

[42] Ghiradella, H. Structure of butterfly scales: Patterning in an insect cuticle. $M i$ crosc. Res. Techniq. 27, 429-438 (1994).

[43] Ghiradella, H. \& Butler, M. Many variations on a few themes: A broader look at development of iridescent scales (and feathers). J. Roy. Soc. Interface 6, S243S251 (2009).

[44] Ingram, A. L. Butterfly photonics: Form and function. In Gorb, S. N. (ed.) Functional surfaces in biology: Little structures with big effects (Springer, 2009).

[45] Yoshioka, S., Nakano, T., Nozue, Y. \& Kinoshita, S. Coloration using higher order optical interference in the wing pattern of the Madagascan sunset moth. J. Roy. Soc. Interface 5, 457 (2008).

[46] Yoshioka, S. \& Kinoshita, S. Polarization-sensitive color mixing in the wing of the Madagascan sunset moth. Opt. Express 15, 2691-2701 (2007). 
[47] Vukusic, P., Sambles, R., Lawrence, C. \& Wakely, G. Sculpted-multilayer optical effects in two species of Papilio butterfly. Appl. Opt. 40, 1116-1125 (2001).

[48] Sweeney, A., Jiggins, C. \& Johnsen, S. Insect communication: Polarized light as a butterfly mating signal. Nature 423, 31-32 (2003).

[49] Cronin, T., Shashar, N. \& Caldwell, R. Polarization vision and its role in biological signaling. Integr. Comp. Biol. 170, 533-543 (2003).

[50] Berthier, S., Charron, E. \& Silva, A. D. Determination of the cuticle index of the scales of the iridescent butterfly Morpho menelaus. Opt. Commun. 228, 349-356 (2003).

[51] Kinoshita, S., Yoshioka, S. \& Kawagoe, K. Mechanisms of structural colour in the Morpho butterfly: Cooperation of regularity and irregularity in an iridescent scale. Proc. Roy. Soc. B - Biol. Sci. 269, 1417 (2002).

[52] Potyrailo, R. et al. Morpho butterfly wing scales demonstrate highly selective vapour response. Nat. Photonics 1, 123-128 (2007).

[53] Plattner, L. Optical properties of the scales of Morpho rhetenor butterflies: Theoretical and experimental investigation of the back-scattering of light in the visible spectrum. J. Roy. Soc. Interface 1, 49 (2004).

[54] Lee, D. \& Lowry, J. Physical basis and ecological significance of iridescence in blue plants. Nature 254, 50-51 (1975).

[55] Parker, A. 515 million years of structural colour. J. Opt. A: Pure Appl. Opt. 2, R15-R28 (2000).

[56] Rutowski, R. L. et al. Iridescent ultraviolet signal in the orange sulphur butterfly (Colias eurytheme): Spatial, temporal and spectral properties. Biol. J. Linn. Soc. London 90, 349-364 (2007).

[57] Kemp, D. J. Female butterflies prefer males bearing bright iridescent ornamentation. Proc. R. Soc. B - Biol. Sci. 274, 1043-1047 (2007). 
[58] Morehouse, N. I., Vukusic, P. \& Rutowski, R. Pterin pigment granules are responsible for both broadband light scattering and wavelength selective absorption in the wing scales of pierid butterflies. Proc. R. Soc. B - Biol. Sci. 274, 359-366 (2007).

[59] Parker, A. \& Hegedus, Z. Diffractive optics in spiders. J. Opt. A: Pure Appl. Opt. 5, S111-S116 (2003).

[60] Kettler, J. The compact-disk as a diffraction grating. Am. J. Phys. 59, 367-368 (1991).

[61] Noda, K., Glover, B., Linstead, P. \& Martin, C. Flower color intensity depends on specialized cell-shape controlled by a MYB-related transcription factor. Nature 369, 661-664 (1994).

[62] Gorton, H. \& Vogelmann, T. Effects of epidermal cell shape and pigmentation on optical properties of Antirrhinum petals at visible and ultraviolet wavelengths. Plant. Physiol. 112, 879-888 (1996).

[63] Hebant, C. \& Lee, D. Ultrastructural basis and developmental control of blue iridescence in Sellaginella leaves. Am. J. Bot. 71, 216-219 (1984).

[64] Vogelmann, T. Plant-tissue optics. Annu. Rev. Plant Physiol. Plant Mol. Biol. 44, 231-251 (1993).

[65] Green, P. B. \& Linstead, P. A procedure for SEM of complex shoot structures applied to the inflorescence of snapdragon (Antirrhinum). Protoplasma 158, 33-38 (1990).

[66] Palmer, C. A. \& Loewen, E. G. E. G. Diffraction grating handbook (Newport Corporation, Rochester, NY, 2005), 6 edn.

[67] Skorupski, P., Doring, T. F. \& Chittka, L. Photoreceptor spectral sensitivity in island and mainland populations of the bumblebee, Bombus terrestris. J. Comp. Physiol. A 193, 485-494 (2007). 
[68] Rutowski, R., Macedonia, J., Morehouse, N. \& Taylor-Taft, L. Pterin pigments amplify iridescent ultraviolet signal in males of the orange sulphur butterfly, Colias eurytheme. Proc. R. Soc. B - Biol. Sci. 272, 2329-2335 (2005).

[69] Kevan, P., Chittka, L. \& Dyer, A. Limits to the salience of ultraviolet: Lessons from colour vision in bees and birds. J. Exp. Biol. 204, 2571-2580 (2001).

[70] Daumer, K. Blumenfarben, wie sie die Bienen sehen. Z. Vergl. Physiol. 41, 49-110 (1958).

[71] Chittka, L. The color hexagon - a chromaticity diagram based on photoreceptor excitations as a generalized representation of color opponency. J. Comp. Physiol. A. 170, 533-543 (1992).

[72] Dyer, A. \& Chittka, L. Fine colour discrimination requires differential conditioning in bumblebees. Naturwissenschaften 91, 224-227 (2004).

[73] Whitney, H. M., Dyer, A., Chittka, L., Rands, S. A. \& Glover, B. J. The interaction of temperature and sucrose concentration on foraging preferences in bumblebees. Naturwissenschaften 95, 845-850 (2008).

[74] Jokiel, P. \& York, R. Importance of ultraviolet-radiation in photoinhibition of microalgal growth. Limnol. Oceanogr 29, 192-199 (1984).

[75] Kay, Q., Daoud, H. \& Stirton, C. Pigment distribution, light-reflection and cell structure in petals. Bot. J. Linn. Soc. 83, 57-83 (1981).

[76] Kevan, P. \& Lane, M. Flower petal microtexture is a tactile cue for bees. Proc. Natl. Acad. Sci. USA 82, 4750-4752 (1985).

[77] Heuschen, B., Gumbert, A. \& Lunau, K. A generalised mimicry system involving angiosperm flower colour, pollen and bumblebees' innate colour preferences. Plant Syst. Evol. 252, 121-137 (2005).

[78] Kevan, P. \& Backhaus, W. Color vision: Ecology and evolution in making the best of the photonic environment. In Backhaus, W., Kliegl, R. \& Werner, J. S. (eds.) 
Color vision: Perspectives from different disciplines, 163-168 (Walter de Gruyter, Berlin, 1998).

[79] Shawkey, M. \& Hill, G. Carotenoids need structural colours to shine. Biol. Lett. 1, 121-124 (2005).

[80] Prum, R. O. Anatomy, physics, and evolution of structural colors. In Hill, G. E. \& McGraw, K. J. (eds.) Bird Coloration: Mechanisms and measurements, vol. 1, 295-353 (Harvard Univ. Press, Boston, 2006).

[81] Gronquist, M. et al. Attractive and defensive functions of the ultraviolet pigments of a flower (Hypericum calycinum). Proc. Natl. Acad. Sci. USA 98, 13745 (2001).

[82] Arnold, S., Savolainen, V. \& Chittka, L. FReD: The floral reflectance spectra database. Nature Precedings (2008). 


\section{Chapter 3}

\section{Materials and techniques}

In this chapter, the materials relevant to this thesis are introduced. Furthermore, sample preparation and characterisation techniques that found application in the context of the thesis are briefly discussed.

\subsection{Materials}

\subsubsection{Polymer materials}

\section{Teflon ${ }^{\circledR}$ AF}

Teflon ${ }^{\circledR}$ AF is a class of amorphous perfluorinated polymers, manufactured by Dupont, which are known for their outstanding chemical resistance, thermal stability, and optical properties in thin films, but also for a remarkable hydrophobicity [1]. Teflon ${ }^{\circledR} \mathrm{AF}$ polymers are co-polymers of tetrafluoroethylene (TFE) and 2,2-bistrifluoromethyl-4,5difluoro-1,3-dioxole (PDD), which retain most of the outstanding physical properties of conventional Teflon (polytetrafluoroethylene, PTFE), while combining them with unique optical, electrical, and solubility characteristics [1]. The structure formula of Teflon ${ }^{\circledR}$ AF is shown in Figure 3.1.

Teflon ${ }^{\circledR}$ AF 1600 and Teflon ${ }^{\circledR}$ AF 2400 are two amorphous fluoro-polymers that are commercially available from DuPont. These materials have a glass transition temperature of $160^{\circ} \mathrm{C}$ and $240^{\circ} \mathrm{C}$, respectively. The glass transition temperature increases with PDD content in the copolymer. Teflon ${ }^{\circledR}$ AF polymers are soluble in fluorinated solvents at room temperature and show a high gas permeability. Teflon ${ }^{\circledR}$ AF polymers are 

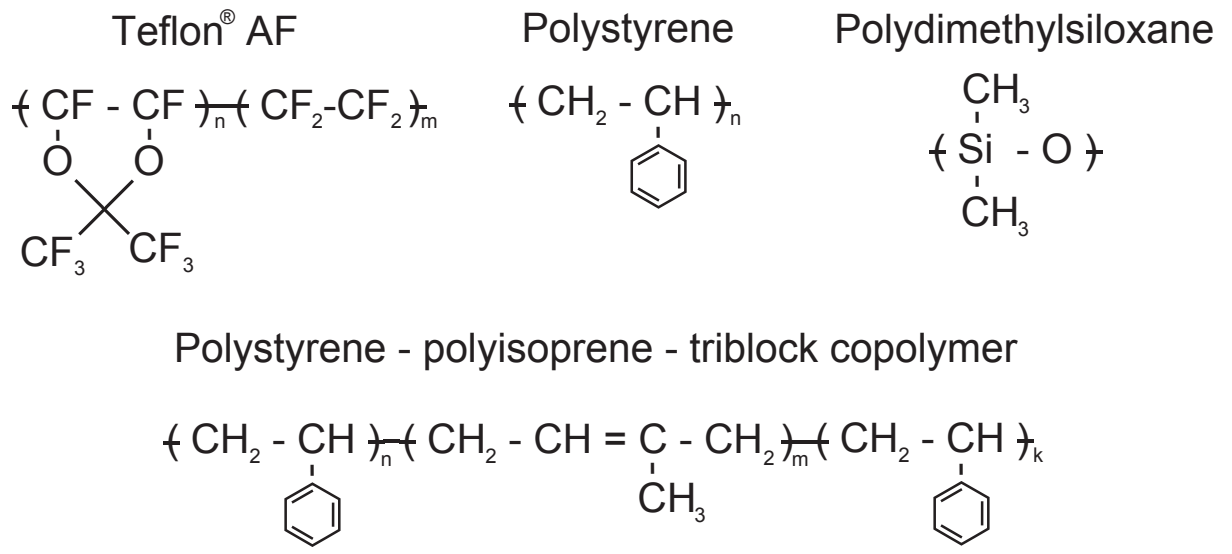

Figure 3.1: Structure formulas of Teflon ${ }^{\circledR} A F$, polystyrene, polydimethylsiloxane and polystyrene-polyisoprene triblock copolymer.

stiffer than PTFE, highly transparent and possess very low refractive indices of around 1.29 - 1.33 which makes them an ideal material for optical applications such as antireflection coatings or interference layers. Commonly available solvents for Teflon ${ }^{\circledR} \mathrm{AF}$ are shown in Table 3.1.

\begin{tabular}{ccc}
\hline Solvent & Boiling point $\left({ }^{\circ} \mathbf{C}\right)$ & Solubility parameter $\left(\sqrt{\frac{\mathrm{J}}{\mathrm{m}^{3}}}\right)$ \\
\hline 'Fluorinert' FC-72 & 60 & 0.0123 \\
Perfluoromethylcyclohexane & 76 & 0.0129 \\
Perfluorobenzene & 82 & 0.0123 \\
Perfluorodimethylcyclohexane & 102 & 0.0139 \\
Perfluorooctane & 103 & 0.0115 \\
'Fluorinert' FC-75 & 103 & 0.0129 \\
Perfluorodecalin & 142 & 0.0135 \\
'Fluorinert' FC-40 & 155 & 0.0135 \\
Perfluoro-1-methyldecalin & 160 & 0.0143 \\
Perfluorodimethyldecalin & 180 & 0.0147 \\
\hline
\end{tabular}

Table 3.1: Solvents for Teflon ${ }^{\circledR}$ AF. From [1].

\section{Polystyrene}

Polystyrene (PS) is one of the industrially most widely used plastic materials. PS with molecular weights $M_{\mathrm{w}}$ of $100,000 \mathrm{~g} / \mathrm{mol}, 200,000 \mathrm{~g} / \mathrm{mol}$, and $300,000 \mathrm{~g} / \mathrm{mol}$ was 
purchased from Sigma Aldrich. The glass transition temperature $T_{\mathrm{g}}$ lies around $100^{\circ} \mathrm{C}$ [2]. PS is a thermoplastic substance. It becomes liquid and can be moulded above its $T_{\mathrm{g}}$, conserving the imposed pattern when cooled below $T_{\mathrm{g}}$. The structural formula of polystyrene is shown in Figure 3.1. Polystyrene is soluble in solvents such as toluene, chloroform, tetrahydrofuran and cyclohexane. The refractive index of PS is $\sim 1.59$ (measured by ellipsometry).

\section{Polystyrene colloids}

Polystyrene colloids were used for deposition of colloidal close-packed monolayers. The aqueous colloid suspensions were purchased from Duke Scientific Standards ${ }^{\top M}$ (part of Thermo Fisher Scientific). The colloids are sterically stabilised and their diameter ranges from $1 \mu \mathrm{m}$ to $5 \mu \mathrm{m}$ with a relative deviation of less than $1 \%$ for each size.

\section{Polydimethylsiloxane}

Polydimethylsiloxane (PDMS) is an industrially widely used rubber material belonging to a group of organosilicon compounds that are known as silicones. PDMS can be cross-linked into networks and is optically transparent. The used PDMS elastomer system, known under the trade name Sylgard ${ }^{\circledR} 184$, was purchased from Dow Corning. It consists of two components, the resin dimethyl methylhydrogen siloxane and the curing agent ethylbenzene. The precursors can be dissolved in toluene or heptane. Typical curing temperatures lie in the range of $60^{\circ} \mathrm{C}$ to $150^{\circ} \mathrm{C}$. The refractive index of PDMS was measured by ellipsometry to be $\sim 1.41$.

\section{Polyisoprene-polystyrene triblock copolymer}

Polystyrene-polyisoprene triblock copolymers (PSPI) are thermoplastic elastomers that are commonly used in industry. These block copolymers consist of polyisoprene chains with covalently linked polystyrene blocks on both chain ends. PSPI with a PS content of 22 wt\% (Sigma Aldrich) was used but other PSPI-polymers with lower weight fractions 
of PS $(14 \mathrm{wt} \%, 17 \mathrm{wt} \%)$ are also available. Compared to pure polyisoprene, PSPI offers similar flexibility, high traction, and sealing properties. PSPI can be readily processed by spin coating from solution in toluene, chloroform, or tetrahydrofuran. A crosslinking step is not required, since the micro-phase separation of the block-copolymer leads to the formation of glassy PS domains in a rubbery PI matrix. These domains act as physical cross-linking sites. The refractive index of the PSPI used in this study has been measured in an ellipsometer giving $\sim 1.54$.

\section{Dental wax}

Elite HD + Light Body Normal Set, distributed by Zhermack, is a hydrophilic silicone material (vinylpolysiloxane) of medium viscosity used for precision impression in dental surgery. The two components are thoroughly mixed in a volume ratio of 1:1. Polymerisation and setting occurs within ten minutes, resulting in a flexible, elastic, biocompatible material with high yield strength.

\section{Organic dyes}

Semiconducting conjugated organic polymer dyes can be used as gain media in microresonators to build optically and electrically pumped LEDs [3-6]. In the context of this thesis, Poly (perylene bisimide acrylate) and Poly(9,9-dioctylfluorene-co-benzothiadiazole) (F8BT) were incorporated in the defect layers of planar polymer microcavities.

Poly (perylene bisimide acrylate) is a side chain perylene bisimide homopolymer which is used as an electron acceptor in organic solar cells [7]. This material was synthesized by Dr. Michael Sommer [8] and its electronic and optical properties have been extensively studied by Dr. Sven Hüttner [7]. Thin dye films can be produced by spin-coating from solution in chloroform.

Poly (9,9-dioctylfluorene-co-benzothiadiazole) was obtained from Dr. Dinesh Kabra from the Optoelectronics group at the Cavendish Laboratories. F8BT is a conjugated polymer with low stimulated emission threshold widely applied in organic optoelectronics 
$[9,10]$ exhibiting photoluminescence emission in the green spectral range. F8BT can be cast into thin layers by spin-coating from a solution in p-xylene. Films should be prepared in an inert atmosphere (nitrogen or argon), avoiding contact with air and water which can cause photo-oxidation [9].
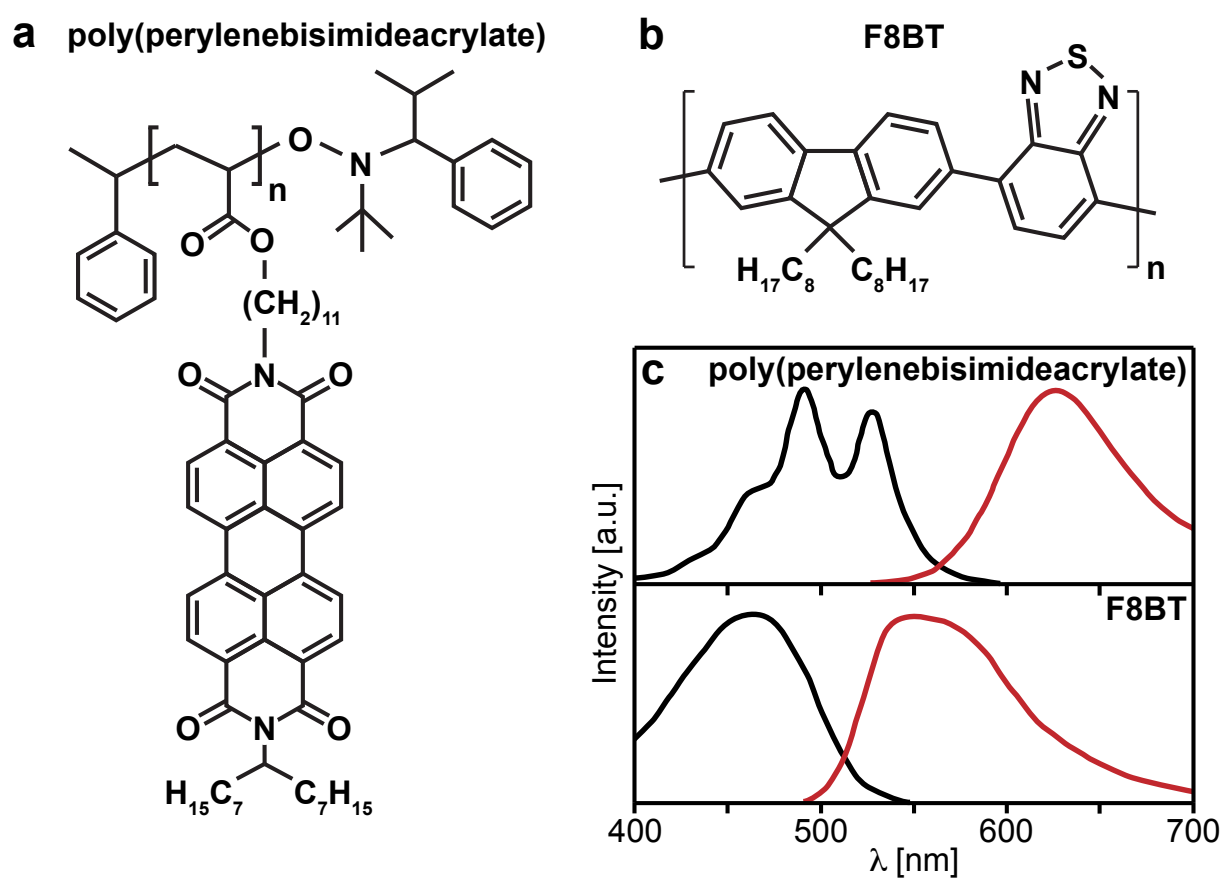

Figure 3.2: Organic dyes. a) Poly(perylene bisimide acrylate). b) Poly(9,9dioctylfluorene-co-benzothiadiazole). c) Absorption (black) and photoluminescence (red) spectra of poly(perylenebisimideacrylate) (top, taken from [7]) and F8BT (bottom, taken from [10]).

\subsubsection{Metal oxides}

\section{Aluminium oxide}

Alumina $\left(\mathrm{Al}_{2} \mathrm{O}_{3}\right)$ is the metal oxide of aluminium. In its crystalline state it is also known as corundum or sapphire. Aluminium oxide is transparent in thin layers and possesses a refractive index of 1.55-1.77 depending on the production technique [11-13]. In the scope of this work, amorphous $\mathrm{Al}_{2} \mathrm{O}_{3}$ was deposited by atomic layer deposition (ALD) from trimethyl-aluminium $\left(\mathrm{Al}\left(\mathrm{CH}_{3}\right)_{3}\right)$ and water at temperatures ranging from $80^{\circ} \mathrm{C}$ to $300^{\circ} \mathrm{C}$. The refractive index of the ALD-deposited aluminium oxide lies in the 
range of $1.60-1.65$, as determined by ellipsometry.

\section{Titanium(IV)oxide}

Titania $\left(\mathrm{TiO}_{2}\right)$ is the naturally occurring oxidation state of the metal titanium. Thin films of titanium oxide can be created via sol-gel chemistry [14], chemical vapour deposition [15], or atomic layer deposition [16]. Titania is usually amorphous if deposited at low temperatures but can be transformed into various crystalline phases upon heating $[17,18]$. The refractive index of $\mathrm{TiO}_{2}$ deposited via ALD in a temperature range of $80-300^{\circ} \mathrm{C}$ lies between $2.43-2.55$, as determined by ellipsometry.

\subsection{Techniques - multilayer production}

\subsubsection{Sequential spin-coating}

Spin-coating is a technique used to cast a thin polymer film from a solution onto a substrate. A droplet of the solution containing the polymer is deposited on the substrate which is subsequently rotated at speed between 500 - 10000 revolutions per minute (rpm). The polymer spreads on the substrate and excess material is flung off, leaving a thin liquid film on the sample, which decreases in thickness as the remaining solvents evaporates, leaving a thin polymer film on the substrate. The remaining solvent is evaporated by post-annealing the polymer film on a hot plate. The process works best if the solvent is a good solvent for the polymer and has a low evaporation rate. The thickness of the resulting polymer film depends on the concentration of the initial polymer solution, the spin speed, the evaporation rate of the solvent and to a certain extent on temperature and humidity in the spin coater [19-22].

Optical multilayer components can be produced by sequential spinning of alternating layers of different polymers with appropriate optical properties. The solvents have to be mutually orthogonal, that is they have to be a good solvent for only one of the polymers but should not dissolve any of the other multilayer components. Furthermore, 
the solutions should reasonably wet the layers onto which they are cast to enable the deposition of a thin layer of the dissolved polymer. Here, we used Teflon ${ }^{\circledR}$ AF dissolved in FC-75 and polystyrene in toluene as a binary orthogonal polymer-solvent system to produce multilayer reflectors and cavities. A complication results from the fact that the Teflon ${ }^{\circledR}$ AF layers are not well wetted by toluene making it impossible to deposit a polystyrene layer by spin coating on top of a Teflon ${ }^{\circledR} \mathrm{AF}$ surface without prior surface treatment. Such a treatment can be achieved by a very short etch of the Teflon ${ }^{\circledR} \mathrm{AF}$ surface in an oxygen plasma to create hydroxyl (OH) groups on the Teflon surface which increases its surface free energy, allowing the deposition of a polystyrene layer. A typical etching step is done in an oxygen atmosphere at a pressure of $\sim 3$ mbar for 0.8-1.2 s in a Diener Electronics Femto plasma etcher (nominal power $100 \mathrm{~W}$ ).

\subsubsection{Floating and stacking}

The release of a thin polymer film from a substrate or the transfer of such a film onto another support is a common problem in polymer processing. This transfer can be done by floating the polymer layer onto the surface of a liquid and subsequently picking it up with another support (Fig. 3.3). A thin sacrificial layer under the polymer film, made of a material that dissolves or degrades in the liquid can facilitate the release of the layer. Poly(4-styrene-sulfonic acid) (PSS) is a good material for this sacrificial layer. Alternatively, a thin gold layer $(\sim 10-30 \mathrm{~nm})$ under the polymer film can be degraded

a

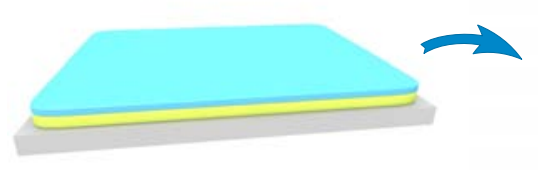

b

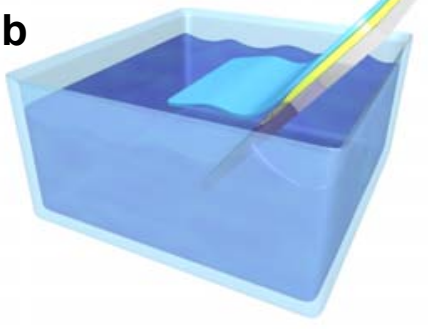

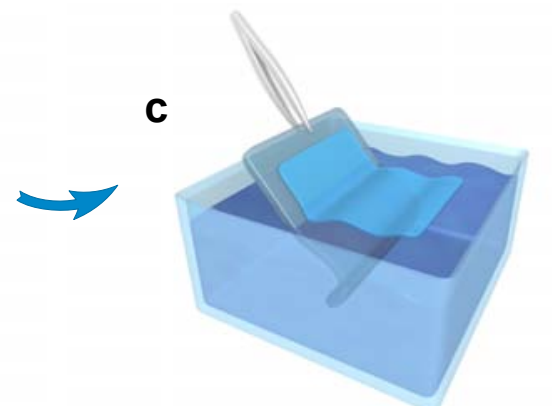

Figure 3.3: The floating technique. a) Thin polymer layer (blue) on a sacrificial film (yellow), deposited on a hard substrate by spin-coating. b) Floating of the polymer layer. The sacrificial film dissolves in the liquid, facilitating the release of the polymer layer. c) Capture of the thin polymer layer on a support. 
in a mild bromine etch [23], allowing the release of the polymer layer. Polymer layers deposited on a glass, silicon or mica substrate can often be released onto a water surface without any sacrificial layer.

The floating technique is used to sequentially stack layers of different polymer materials. This method can also be applied to produce multilayer stacks by float-folding [24] (Chapter 4).

\subsubsection{Atomic layer deposition}

Atomic layer deposition (ALD), also called molecular layer epitaxy, is a modified type of chemical vapour deposition (CVD) involving highly reactive precursors with great mutual affinity [25]. As opposed to standard CVD processes the reaction partners are sequentially and separately introduced into the reaction chamber [26]. While in a CVD process, the reaction happens in the gas phase and the reaction products adsorb on the sample, in an ALD process the reaction can only take place at the sample surface. This allows the deposition of coatings onto irregular, porous or overhanging surface morphologies and other very high aspect ratio structures with extremely good surface conformity [23, 27-35] (Fig. 3.4).
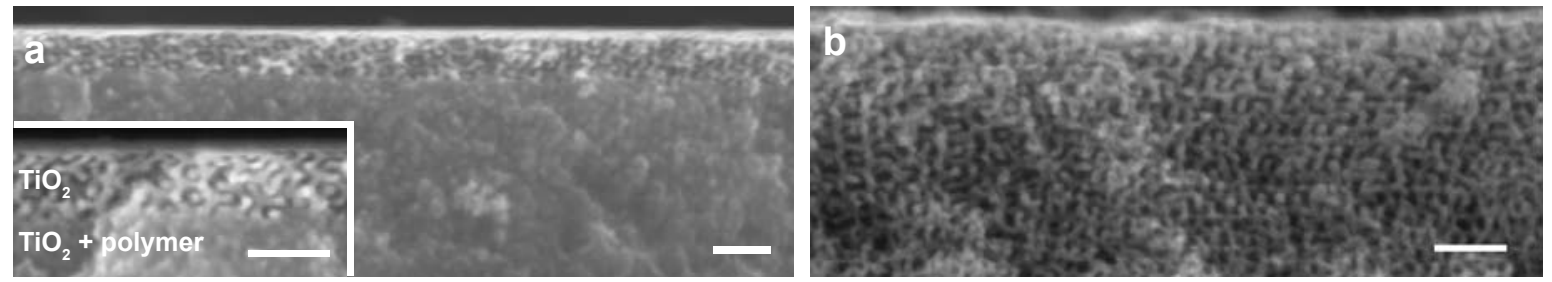

Figure 3.4: $\mathrm{TiO}_{2}$ gyroids produced by ALD. a) Cross sectional SEM image of a polymer gyroid template, filled with $\mathrm{TiO}_{2}$ by $\mathrm{ALD}$ at $80^{\circ} \mathrm{C}$, after exposure to an oxygen plasma for $5 \mathrm{~min}$ to etch the polymer. In the top part of the film the $\mathrm{TiO}_{2}$ gyroid is revealed, while the bottom part still contains the polymer template. b) The freestanding $\mathrm{TiO}_{2}$ gyroid network after complete removal of the polymer template. The gyroid polymer templates were fabricated by Dr. Ed Crossland [23] and the ALD deposition was done with the help of Dr. Robin Ras and Dr. Jari Malm at the Helsinki University of Technology. All scale bars are $100 \mathrm{~nm}$. 
Materials that can be deposited include metal oxides [36-39], metal nitrides [25], metals $[40,41]$ but also some polymers, such as polyimides $[42,43]$. Low refractive index metal fluoride thin films, such as $\mathrm{CaF}_{2}$ or $\mathrm{MgF}_{2}$, for applications in antirefelction coatings and optical filters can also be produced by atomic layer deposition [44, 45]. The growth of silver films by $\mathrm{ALD}$ at $140^{\circ} \mathrm{C}$, which could be suitable for plasmonic applications, has been reported recently [46]. Usually, the precursors are thermally activated [25]. Thermal ALD processes take place between $100-300^{\circ} \mathrm{C}$ but some materials, like titania or alumina, can be grown at temperatures as low as $80^{\circ} \mathrm{C}$, which is especially important for deposition on polymer substrates [47].

Plasma-enhanced ALD is more suitable for low temperature processes (as low as room temperature) and also allows water to be avoided as a precursor in the case of metal oxide depositions [40, 48, 49]. The necessary activation of the second precursor, which is usually oxygen for metal oxides or a nitrogen/ammonia mixture for metal nitrides, happens by ionisation of the molecules in a remote plasma that is not in contact with sample. The surface chemistry on the substrate has a strong influence on the growth of the first atomic layers [25, 50]. For the deposition of metal oxides, a high abundance of hydroxyl groups on the substrate surface allows for good chemisorption of the reactive precursor molecules resulting in a faster growth of a dense first atomic layer [51].

A typical thermal ALD process is shown schematically in Figure 3.5. One ALD cycle consists normally of at least four steps involving two different precursor materials. At the beginning of each cycle the first precursor material, for example a metal halide, is introduced into the reaction chamber and adsorbs at the substrate surface. In an ideal ALD process the adsorption of the precursor is self-terminating so that no more precursor molecules can permanently adsorb on the surface once a monolayer is completed. In the second step non-adsorbed precursor molecules are purged off the surface and out of the chamber in an inert gas flush leaving only a monolayer of the precursor molecules on the substrate. Subsequently, the second precursor material is released into the chamber reacting with the first precursor to form a complete monolayer on the substrate surface. The cycle concludes with an inert gas flush to purge away remaining molecules of the second precursor and the reaction by-products. 


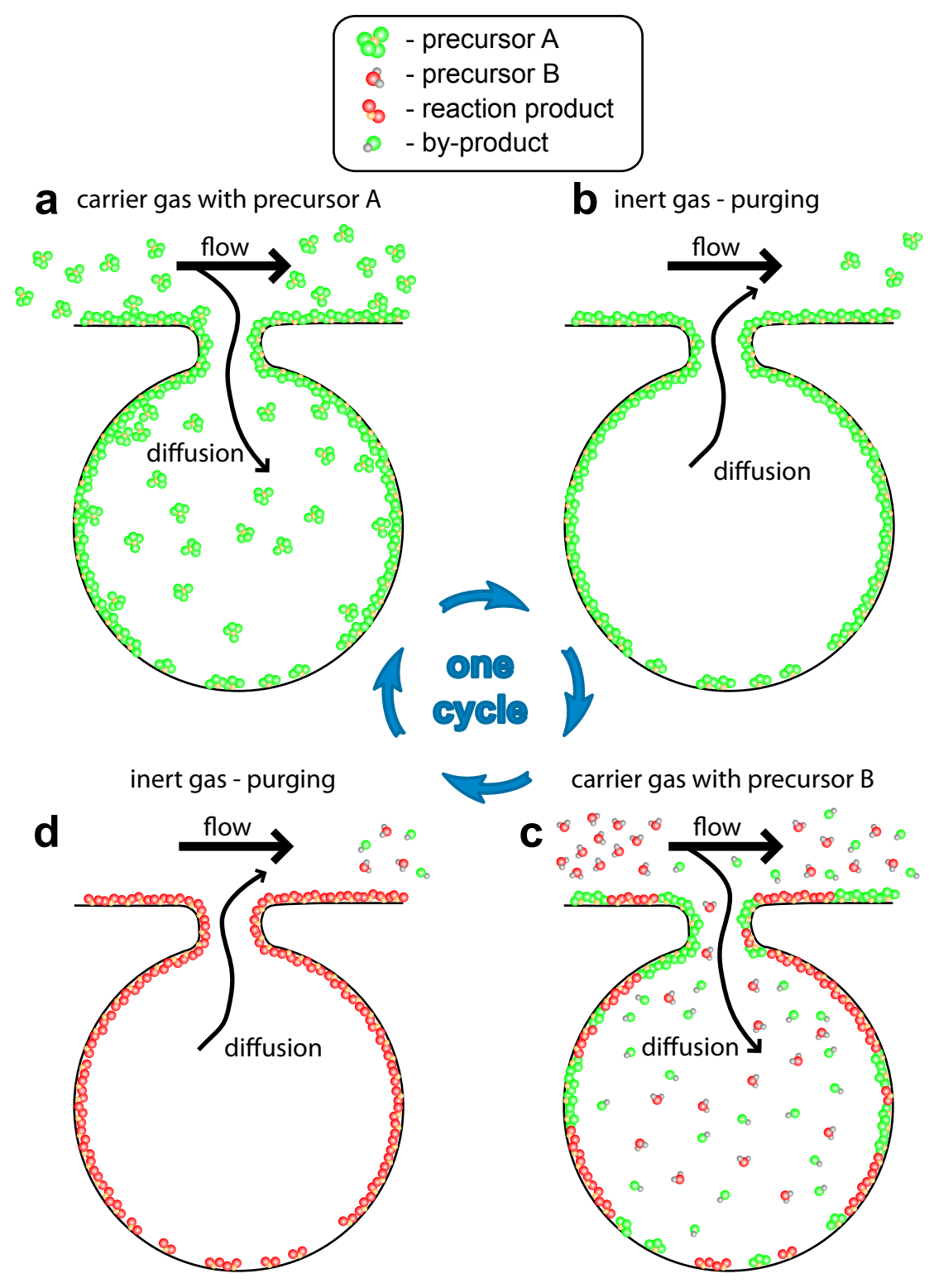

Figure 3.5: A typical atomic layer deposition cycle. a) Precursor $A$ is introduced into the reaction chamber and deposits on the sample. b) A purging step with inert gas removes the non-adsorbed precursor molecules, leaving only a monolayer of precursor $A$ on the sample surface. c) Introduction of precursor B into the reaction chamber. $B$ reacts with the adsorbed molecules on the surface transforming the monolayer of precursor $A$ into a monolayer of the final, stable reaction product $C$ under formation of the reaction by-products. d) A purging step with inert gas removes the non-reacted precursor B molecules and the by-products from the chamber. This cycle is repeated to grow a conformal coating - monolayer by monolayer. The duration of each step in one deposition cycle has to be adapted to the complexity of the structure to allow for diffusion of precursor into the cavities of a structured surface during precursor pulsing and diffusion of non-adsorbed or non-reacted precursor and by-products out of the structure during purging. 

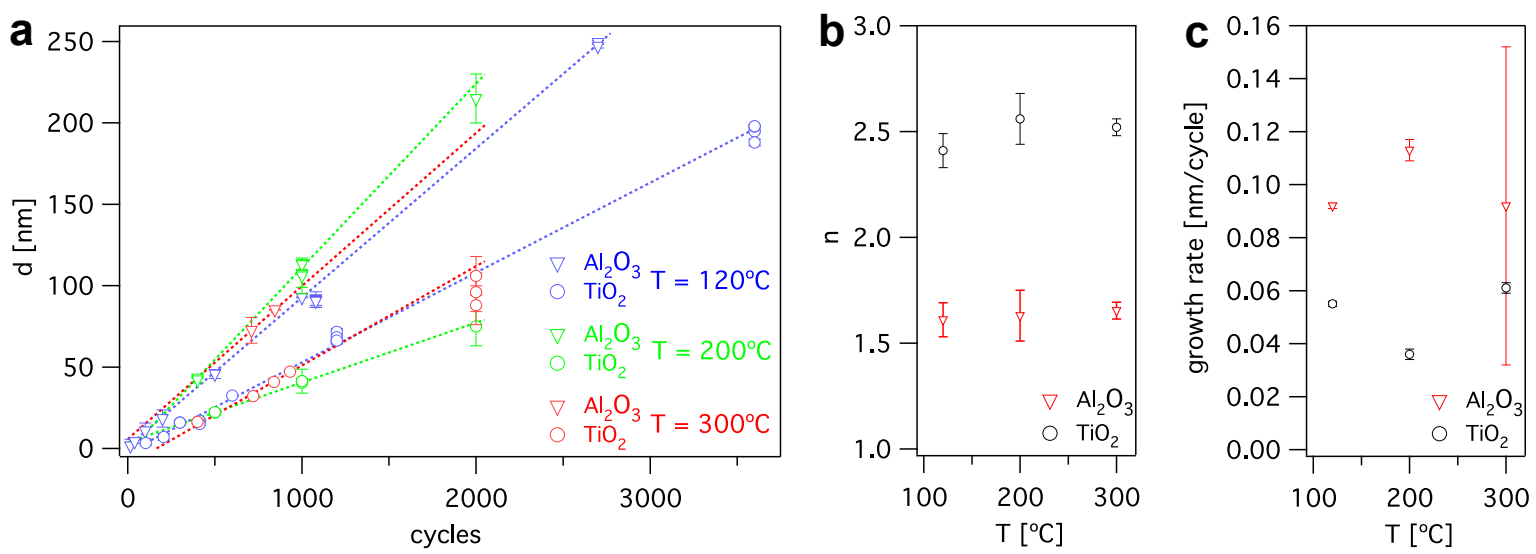

Figure 3.6: Titania- and alumina-deposition curves. a) Film thickness versus cycle number for depositions of $\mathrm{TiO}_{2}$ and $\mathrm{Al}_{2} \mathrm{O}_{3}$ at $120^{\circ} \mathrm{C}, 200^{\circ} \mathrm{C}$ and $300^{\circ} \mathrm{C}$. b) Average refractive index of the layers deposited as a function of temperature. c) Growth rates as a function of temperature, determined from the slopes of the linear fits in (a).

One ALD cycle ideally results in one monolayer of the reaction product on the surface of the substrate. In practice, different growth mechanisms are observed resulting in growth rates that can be lower or higher than one monolayer per deposition cycle [25]. Ultimately, the final thickness of ALD deposited films is a function of the growth rate, which might depend on the deposition temperature, and the number of deposition cycles. As the reaction relies on the sequential adsorption of precursors on the substrate and only takes place at the surface, high-aspect-ratio structures can be conformally coated as long as the duration of each step in a cycle is adjusted to allow the precursor gases to reach all parts of a convoluted surface by diffusion [52]. For flat surfaces, cycle times on the order of half a second can easily be achieved. However, for high aspect ratio structures cycle times usually range from a couple of seconds to tens of seconds. The precise parameters depend strongly on the type of ALD reactor and can vary significantly from system to system.

In this study, a Beneq TFS 200 reactor was used to deposit titania, alumina and zinc oxide (Table 3.2). In Figure 3.6 a the layer thickness of titania and alumina as a function of ALD cycles is plotted for several deposition temperatures. The refractive indices of the ALD deposited alumina and titania layers, measured by ellipsometry, are $n_{\mathrm{Al}_{2} \mathrm{O}_{3}} \simeq 1.65$ and $n_{\mathrm{TiO}_{2}} \simeq 2.5$, respectively (Fig $3.6 \mathrm{~b}$ ). The growth rate, determined as the increase in layer thickness per ALD cycle is $\sim 1 \AA /$ cycle for the alumina deposition 
and $\sim 0.5 \AA /$ cycle for the growth of $\mathrm{TiO}_{2}$ (Fig. 3.6c).

\begin{tabular}{ccccccc}
\hline Material & \multicolumn{2}{c}{ Precursors } & Substrate & Activation & $\mathbf{T ~ [ { } ^ { \circ } \mathrm { C } ]}$ & $\mathbf{n}$ \\
\hline $\mathrm{TiO}_{2}$ & $\mathrm{TiCl}_{4}$ & $\mathrm{H}_{2} \mathrm{O}$ & Glass, Si, PDMS & thermal & $80-300^{\circ} \mathrm{C}$ & $2.4-2.6$ \\
$\mathrm{Al}_{2} \mathrm{O}_{3}$ & $\mathrm{Al}\left(\mathrm{CH}_{3}\right)_{3}$ & $\mathrm{H}_{2} \mathrm{O}$ & Glass, Si, PDMS & thermal & $80-300^{\circ} \mathrm{C}$ & $1.60-1.65$ \\
$\mathrm{Al}_{2} \mathrm{O}_{3}$ & $\mathrm{Al}\left(\mathrm{CH}_{3}\right)_{3}$ & $\mathrm{O}_{3}$ & Glass, Si & thermal & $200^{\circ} \mathrm{C}$ & 1.7 \\
$\mathrm{Al}_{2} \mathrm{O}_{3}$ & $\mathrm{Al}\left(\mathrm{CH}_{3}\right)_{3}$ & $\mathrm{O}_{2}$ & Glass, $\mathrm{Si}$ & plasma & $120^{\circ} \mathrm{C}$ & - \\
$\mathrm{ZnO}$ & $\mathrm{Zn}\left(\mathrm{C}_{2} \mathrm{H}_{5}\right)_{2}$ & $\mathrm{H}_{2} \mathrm{O}$ & Glass, Si & thermal & $80-200^{\circ} \mathrm{C}$ & $1.8-2.0$ \\
\hline
\end{tabular}

Table 3.2: Metal oxides, deposited by $\mathrm{ALD}$, include titania $\left(\mathrm{TiO}_{2}\right)$ from titanium chloride $\left(\mathrm{TiCl}_{4}\right)$, alumina $\left(\mathrm{Al}_{2} \mathrm{O}_{3}\right)$ from trimethylaluminium $\left(\mathrm{Al}\left(\mathrm{CH}_{3}\right)_{3}\right)$, and zinc oxide $(\mathrm{ZnO})$ from diethylzinc $\left(\mathrm{Zn}\left(\mathrm{C}_{2} \mathrm{H}_{5}\right)_{2}\right)$. Water or ozone are used as the second precursors in thermal ALD processes, and oxygen is the reaction partner in metal oxide deposition by plasma-enhanced ALD. The refractive index of the deposited material usually increases with increasing deposition temperature in thermal ALD processes.

\subsection{Techniques - pattern creation and transfer}

\subsubsection{Colloid templating}

A monolayer of hexagonally arranged micron-sized colloids can serve as a versatile etching or deposition mask [53, 54]. Locally well-arranged colloidal mono- or multilayers of $<1 \mu \mathrm{m}$-sized colloids can be deposited by spin-coating [55, 56], evaporation deposition,
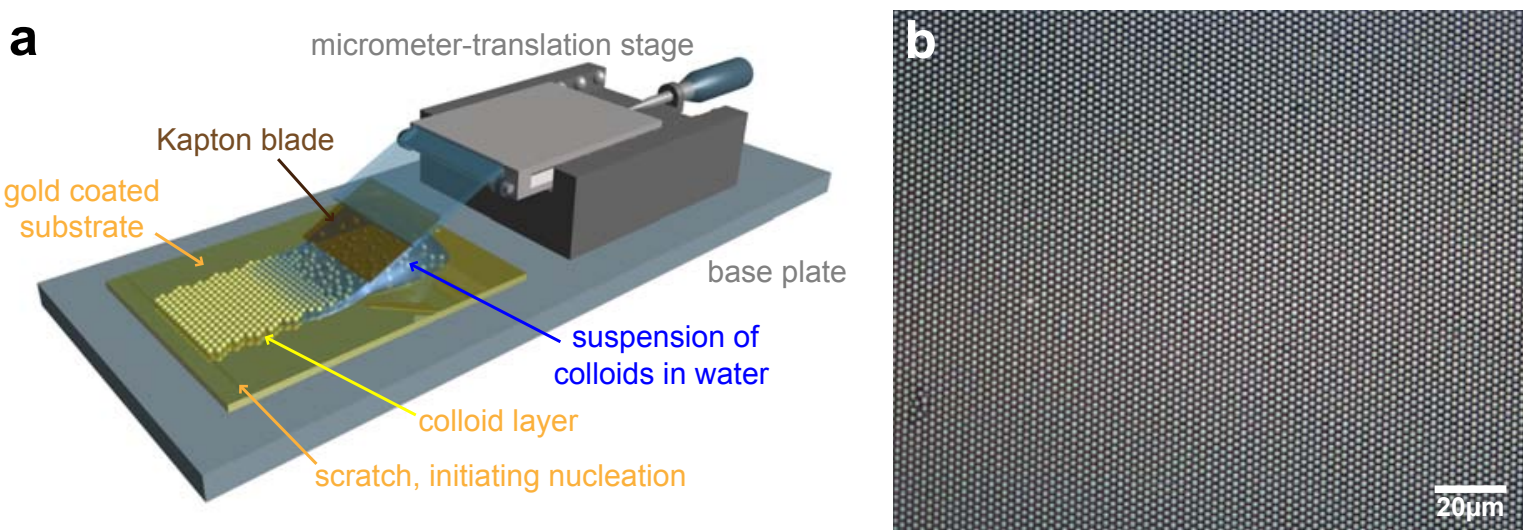

Figure 3.7: Colloidal monolayers. a) Schematic of the colloid templating device. b) Micrograph of a monocristalline colloidal monolayer made of $2 \mu \mathrm{m}$-sized colloids. Picture taken from [60]. 
or in a local confinement $[57,58]$. For larger colloids, these techniques are more difficult to implement. Another approach to deposit colloids in the $1-5 \mu \mathrm{m}$ size range, consists of "blade coating" them onto a flat sample surface. This technique has been described in [59], but has also been developed independently by M. Scherer at the University of Cambridge [60]. A hydrophobic blade is mounted at an angle of roughly $30^{\circ}$ above the sample. The blade can be moved along one sample axis with the help of a micrometer screw (Fig. 3.7 a). A droplet of an aqueous colloid suspension under the blade, forms a meniscus between the blade and the sample surface. The colloids are drawn into the receding meniscus and assemble at the contact line, driven by evaporation of the water and resulting capillary forces which are most pronounced at the meniscus, where the colloids are only partially immersed [61]. Pulling the blade back slowly and at a constant speed results in the deposition of a dense, hexagonally arranged layer of colloids with mono-crystalline regions of several hundred $\mu \mathrm{m}^{2}$ (Fig. $3.7 \mathrm{~b}$ ).

\subsubsection{Argon ion milling}

Argon ion milling is often used to structure metal or inorganic dielectrics. As opposed to reactive ion etching, ion milling consists of a physical dry etch of the sample surface. The technique is based on eroding exposed areas of a sample by bombarding the surface with argon ions.

Argon atoms are ionised in a plasma and an applied bias-potential directs a collimated ion beam towards the sample. Argon ions hitting the sample surface transfer their energy to atoms of the sample surface, which are released from the surface. Argon ion milling takes place under high vacuum to prevent the scattering of the argon ions in the sample atmosphere and to provide a good collimation and directionality of the milling beam. An important parameter in argon ion milling is the beam current, which provides a measure of the number of argon ions created in the plasma during a certain time interval. The acceleration voltage determines the speed, i.e. the energy of the ions, and the standard deviation of ion paths from the average beam direction. The stage current determines the number of Argon ions actually hitting the sample. In 


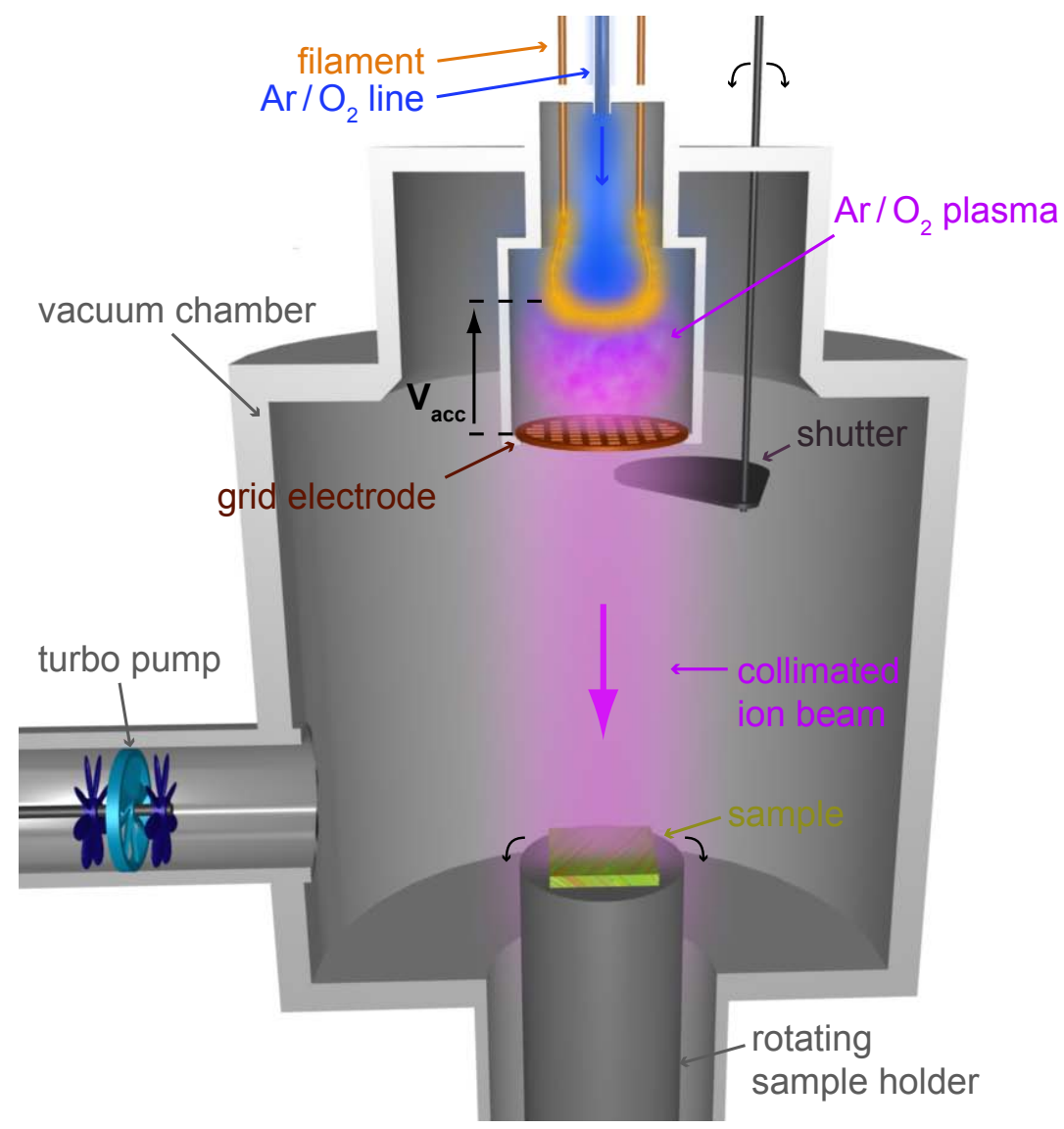

Figure 3.8: Schematic of the Argon ion milling device. A mixture of argon with $\sim 2 \%$ oxygen is ionised in a plasma and directed towards a sample by a bias-potential.

order to account for beam fluctuations or beam inhomogeneities, the sample is rotated with an angular velocity on the order of $1 \mathrm{~min}^{-1}$. The diameter of the ion beam, which determines the maximum area that can be patterned, measures roughly $3 \mathrm{~cm}$ for the used milling system. To protect specific areas of the sample, SU-8, Az, polystyrene or PMMA resists can be used, since the etching rates of these organic coatings are several orders of magnitudes lower than the etching rates of most inorganic materials $[62,63]$. Argon ion milling provides good directionality and with suitable masks high aspect ratios can be achieved. A schematic of the used setup is shown in Figure 3.8.

\subsubsection{Nano-imprint lithography}

Nano-imprint lithography is used to transfer micro- and nano-structures into a polymer film by mechanical deformation. This technique has first been demonstrated by 

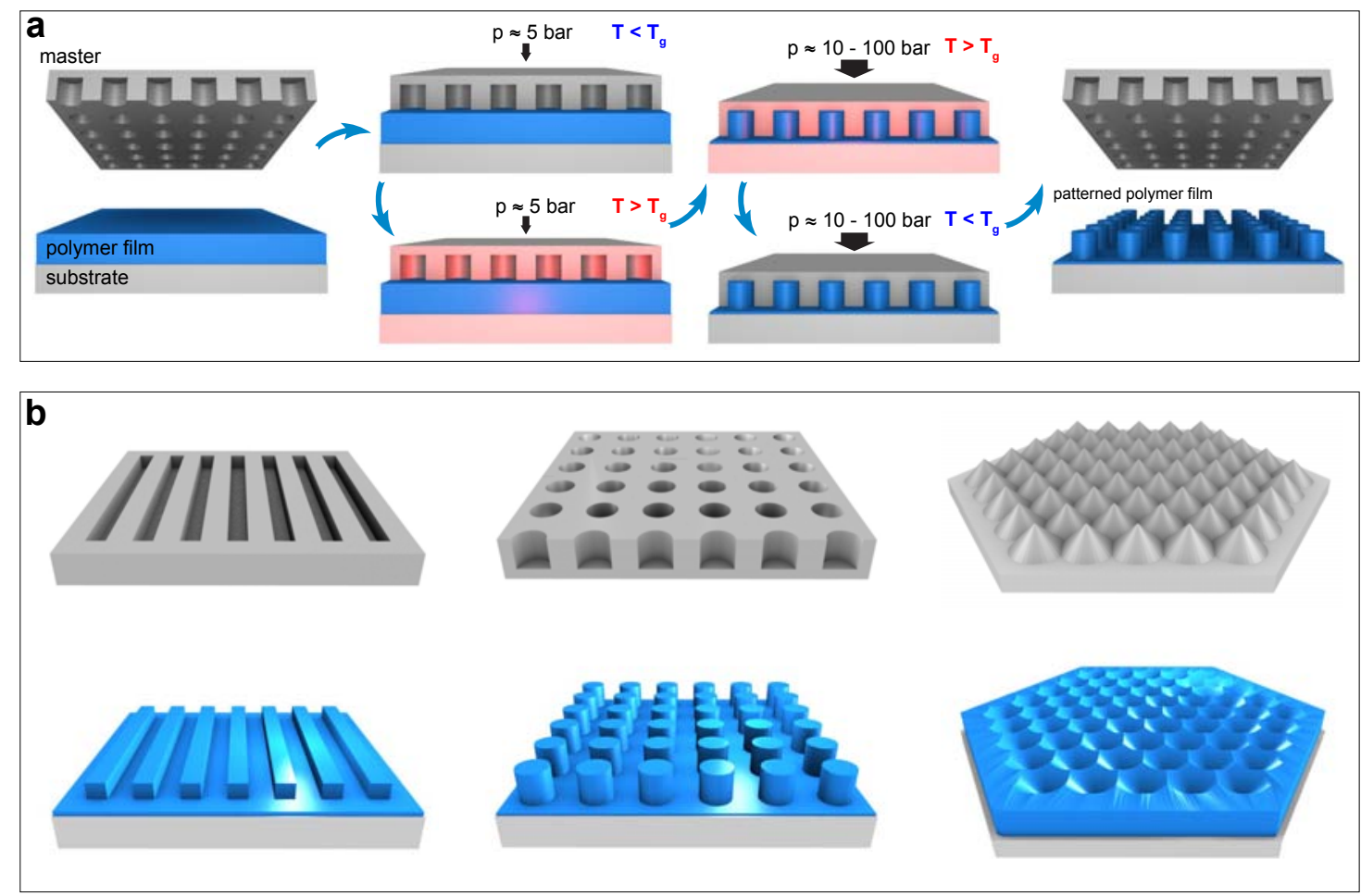

Figure 3.9: Schematic of the nano-imprinting process. a) First, the mould is brought in contact with the polymer film. A moderate pressure is applied and the whole assembly is heated above the glass transition temperature of the polymer. Subsequently a pressure of $10-100$ bar is applied for a timespan of several hundreds of seconds. Afterwards, with the pressure still applied, the assembly is cooled below the glass transition temperature of the polymer, thereby freezing-in the pattern, before the mould is released. b) Schematic of different moulds used in the context of this thesis and the resulting inverse polymer structures.

S. Y. Chou and co-workers $[64,65]$. A schematic of the nano-imprint process is shown in Figure 3.9. A micro- or nano-patterned mould is brought in contact with a thin polymer layer. Heating the assembly above the polymer glass transition temperature $T_{\mathrm{g}}$, softens the polymer material which allows the transfer of a negative of the mould pattern into the polymer film. Contact pressures range from 10 to 100 bar. After holding the assembly at a temperature above $T_{\mathrm{g}}$ for several hundreds of seconds, the assembly is cooled down while maintaining the pressure to freeze-in the pattern in the polymer film. Finally, the mould is separated from the sample leaving the imprinted polymer film, which displays a negative copy of the mould (Fig. 3.9).

In practice, a mask release is often difficult because parts of the patterned polymer film tend to stick to the mould. When using silicon moulds, the mask surface is routinely 
modified by silanization [66], rendering the surface apolar and thus less sticky.

Alternatively, patterned fluorocarbon sheets (ETFE) can be used as flexible moulds, which are intrinsically hydrophobic [67]. These can be produced by imprinting patterned silicon masters into ETFE sheets. The inherent advantage of using ETFE sheets lies in their flexibility and non-stickiness, which simplifies the release process considerably. One sheet can be repeatedly used before a new one has to be produced, greatly reducing the wear and tear on the original silicon master. Alternative masters can be produced from a high temperature epoxy (Epo-Tek OE132) that is coated with silicon oxide and rendered hydrophobic by silanization.

\subsubsection{Dental wax casting}

Natural photonic structures are sometimes difficult to study since they are often hydrated. This complicates spectroscopic experiments involving intense light or electron microscopic studies in high vacuum. In the cases where only the surface of a natural organic structure is of interest, problems due to hydration can be circumvented by taking casts of the nano- or and micro-structures on the surface. A technique common in biology consists in a two-step pattern transfer, relying on quick-setting viscous dental wax. The wax is first cast onto the natural organic structure to produce a negative replica with sub-micrometer resolution (Fig. 3.10). The dental wax replica is then used as a mould to cast a positive replica of the natural original in optical epoxy (Epo-Tek 301).

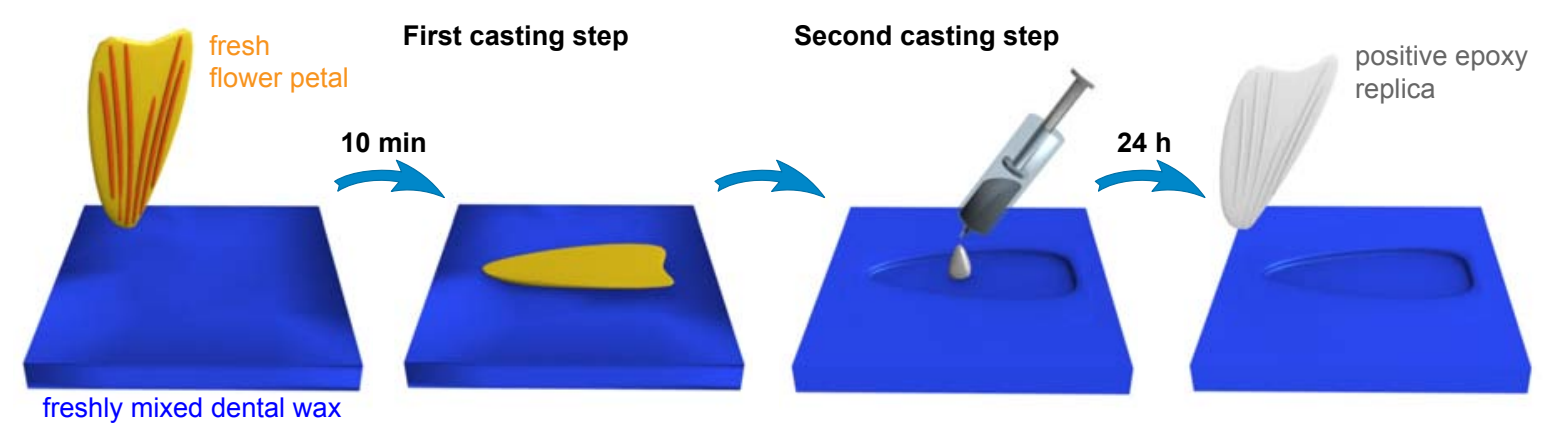

Figure 3.10: Schematic of the two-step pattern transfer using dental wax. First a negative replica of the original structure is cast in dental wax. In a second step, the dental wax cast is used as a mould to create the final positive replica from epoxy. 


\subsection{Techniques - sample characterisation}

A first and very important assessment of the performance of optical structures can often be done by eye or with a light microscope. However, for in-depth characterisation of reflection and scattering behaviour, two different spectroscopic techniques have been used in this work.

\subsubsection{Gonio-spectroscopy}

A detailed characterisation of the reflection, transmission, or diffraction properties of micro- or nano-structured samples is realised by performing spectroscopic measurements at varying light incidence and collection angles.

A suitable spectrometer set-up has been constructed in the context of this thesis. Sample and detector stage can be rotated in steps of $<0.1^{\circ}$, where the incidence and

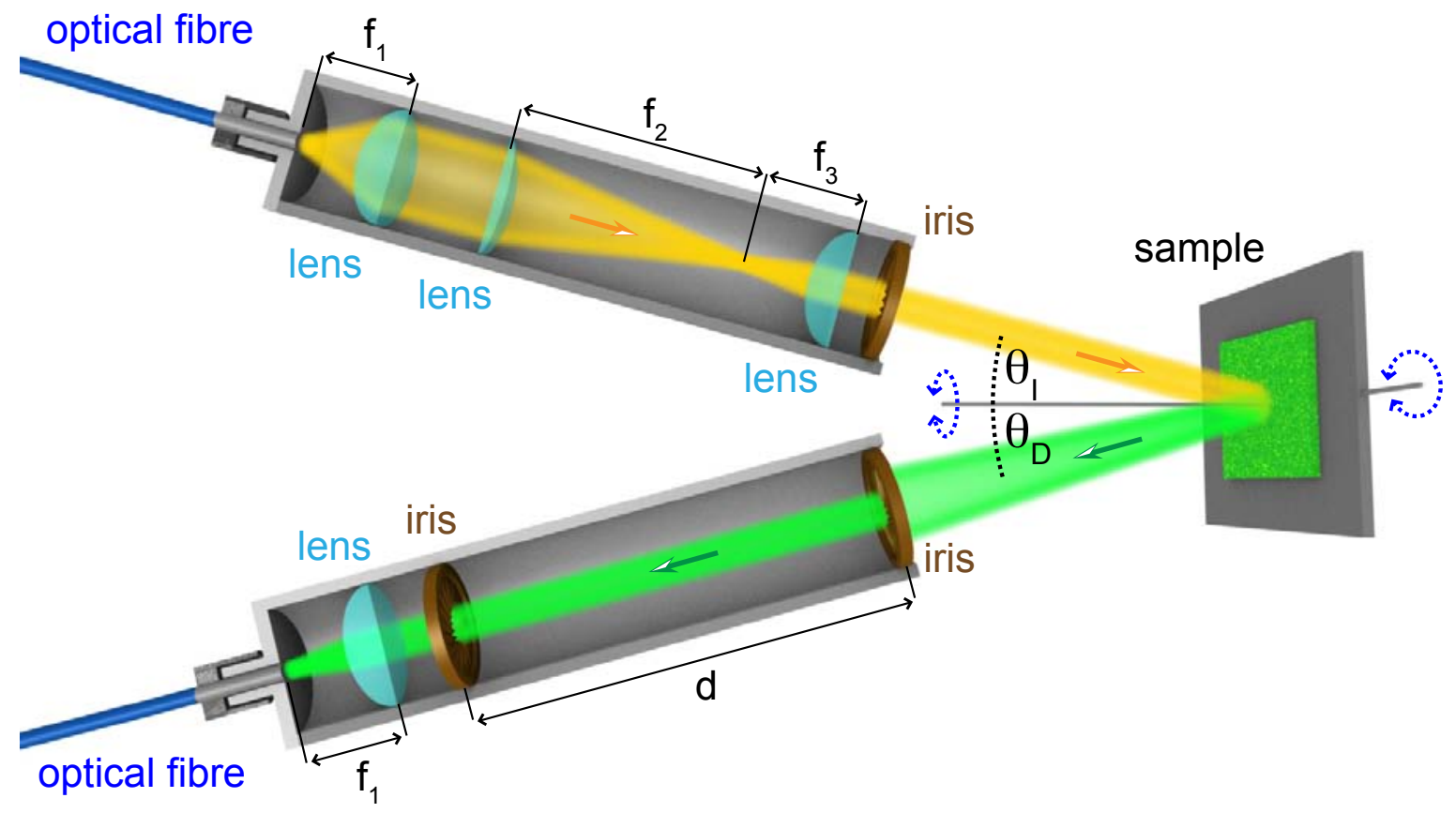

Figure 3.11: Sketch of the gonio-spectroscope. The sample can be rotated perpendicularly to the plane of incidence to adjust the light incidence angle $\theta_{\mathrm{I}}$. Independently the detector is rotated to vary the detection angle $\theta_{\mathrm{D}}$. In addition, the sample can be rotated around its normal to allow interrogation of its orientation-dependent optical anisotropies. 
detection angles can be adjusted independently. This allows measurement of the sample's angularly resolved reflection, transmission, and diffraction in different geometries. A sketch of the set-up is shown in Fig. 3.11. The accessible spectral region lies in the range of 230-880 nm (light source, Ocean Optics 230 - $2000 \mathrm{~nm}$; spectroscope USB 4000, Ocean optics, $200-880 \mathrm{~nm})$.

From the lamp, the light is coupled into a multimode fibre of $600 \mu \mathrm{m}$ or $1000 \mu \mathrm{m}$ core size with numerical aperture of 0.22 . A lens is placed in focal distance $f_{1}$ from the fibre output to collimate the light. Two additional lenses with a focal distance $f_{3}>f_{2}$ are used to reduce the beam cross-section, increasing the intensity per unit area. In the detection unit of the spectroscope the collected light passes through two apertures spaced by about $10 \mathrm{~cm}$ (Fig. 3.12) defining a field of detection of $<1 \mathrm{~mm}^{2}$ and assuring an angular resolution of less than $1^{\circ}$. Subsequently the light is focused onto an optical fibre with $600 \mu \mathrm{m}$ core size which sends the light to the spectroscope.

Various samples have been characterised in four different geometries (Fig. 3.12):

- Specular reflection geometry (SR),

- Backscattering and diffraction geometry (BSD),

- Transmission geometry (T),

- Forward scattering and diffraction geometry (FSD).

For reflection measurements of samples with a strong specular reflection a silver mirror of $>96 \%$ specular reflectance is used as a reference. A white Lambertian reflectance standard (Labsphere Spectralon SRM-99) of $99.9 \%$ diffuse reflectance is used as a reference for samples that show strong diffuse scattering. In transmission measurements the freely propagating light beam serves as reference. 

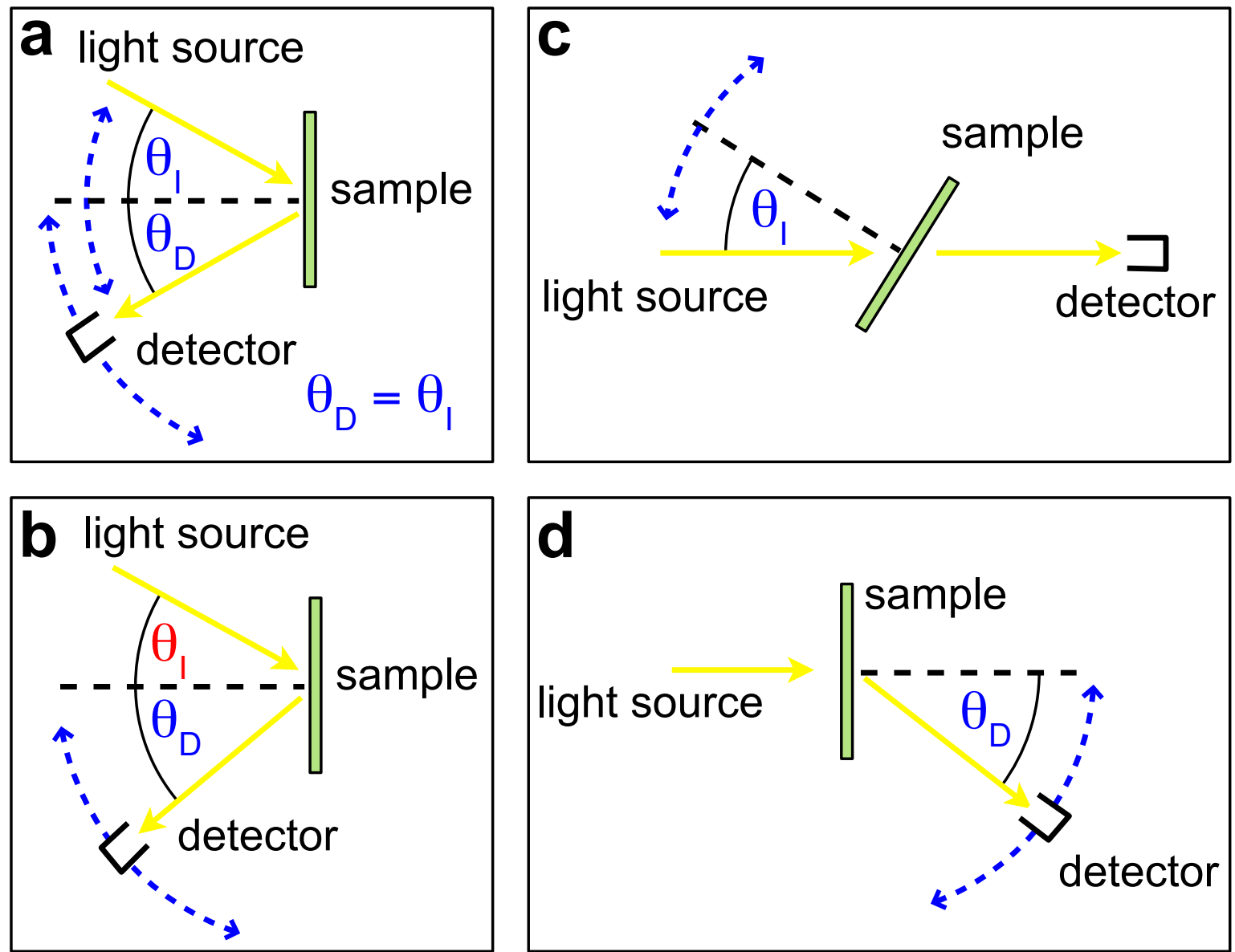

Figure 3.12: Measurement geometries. a) Specular reflection geometry $(\theta-2 \theta$-geometry). b) Backscattering and diffraction geometry. c) Transmission geometry. d) Forward scattering and diffraction geometry. Variable angles are shown in blue, fixed angles in red.

\subsubsection{Micro-spectroscopy}

A modified optical microscope (Olympus BX-51) is used to study the local optical properties of a sample with a resolution of $\sim 1 \mu \mathrm{m}$. The microscope halogen lamp (spectral range $\sim 400-800 \mathrm{~nm}$ ) serves as light source. Alternatively, light of desired spectral range (for example from an external laser) can be coupled into the microscope light path. Light scattered from the sample is coupled out via a beamsplitter and redirected with an optical fibre towards a spectroscope (Fig. 3.13). The fibre diameter and the microscope objective determine the diameter of the spot from which spectra of the sample are acquired. With a $100 \times$ objective and a $50 \mu \mathrm{m}$ core fibre, the detection spot is smaller than $1 \mu \mathrm{m}$. Using a $x$-y-micro-translation stage, the sample can be 


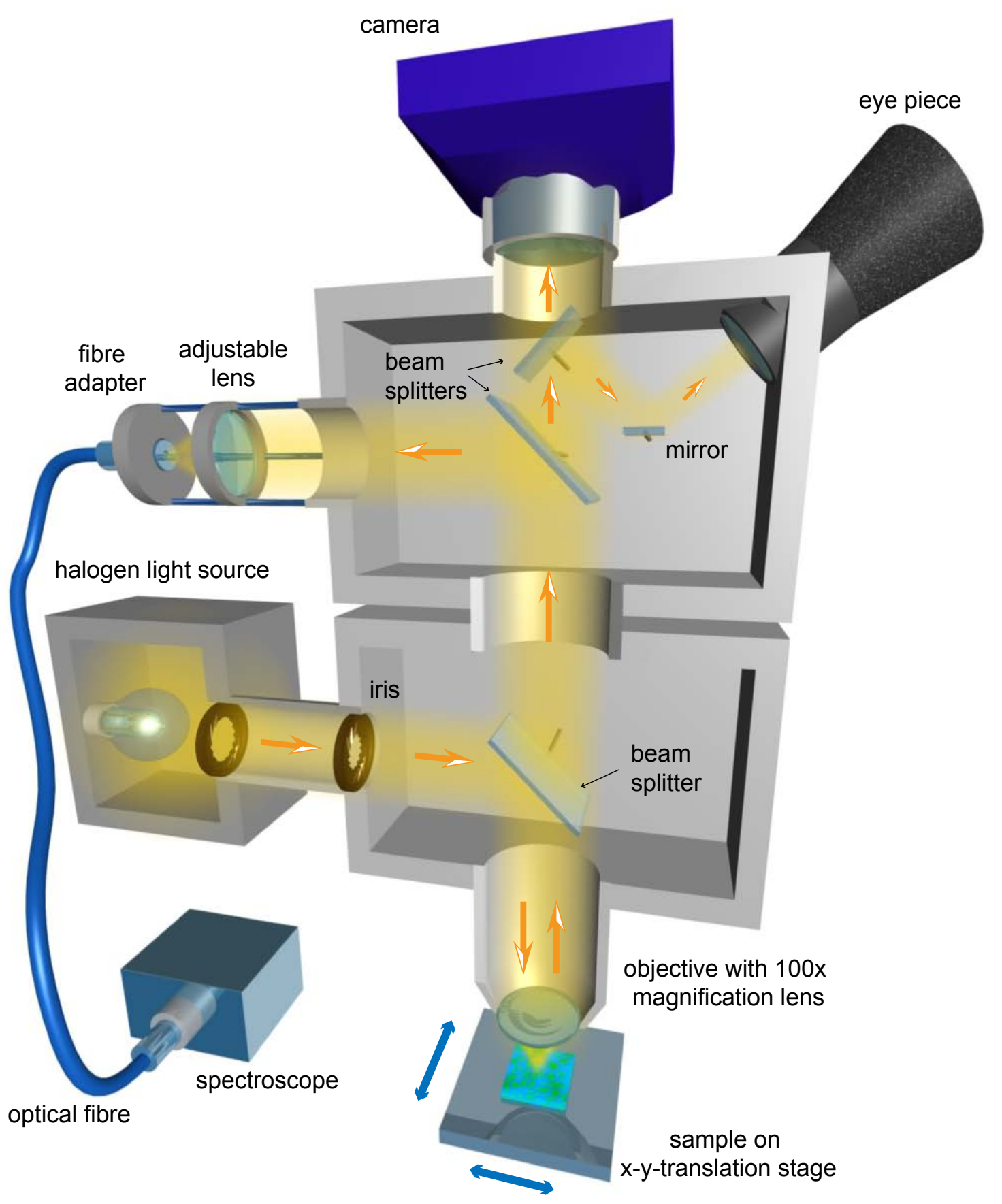

Figure 3.13: Schematic of the micro-spectrometer. Light is directed from the microscope's internal halogen light source via the microscope objective onto the sample. Alternatively, light from an external source such as a laser can be coupled into the main beam path of the microscope. The part of the light that is reflected (or transmitted) from the sample, is redirected via a beamsplitter and an optical fibre to a spectroscope. 
moved in the focal plane to build-up a spectral mapping of the sample's local optical properties. High spatial optical resolution implies a large angular spread in the wave vector distribution of the incident light around the sample normal. This can lead to complications in the characterisation of samples for which reflection and transmission behaviour depends strongly on the light incidence direction (e.g. micro-cavities).

\subsubsection{Ellipsometry}

Ellipsometry is a convenient technique to determine the thickness and complex refractive index of thin films of transparent materials, by analysing the change in the polarisation of light upon reflection from the film's optical interfaces [68, 69]. Light of known elliptical polarisation is sent onto the sample and the polarisation of the reflected light is determined, providing information about the refractive index $n$, absorption coefficient $k$ and thickness $d$ of the thin film. High precision measurements of these parameters can be achieved by using nulling ellipsometry. Light of a given wavelength is sent through a linear polariser and a compensator (a quarter wave plate)

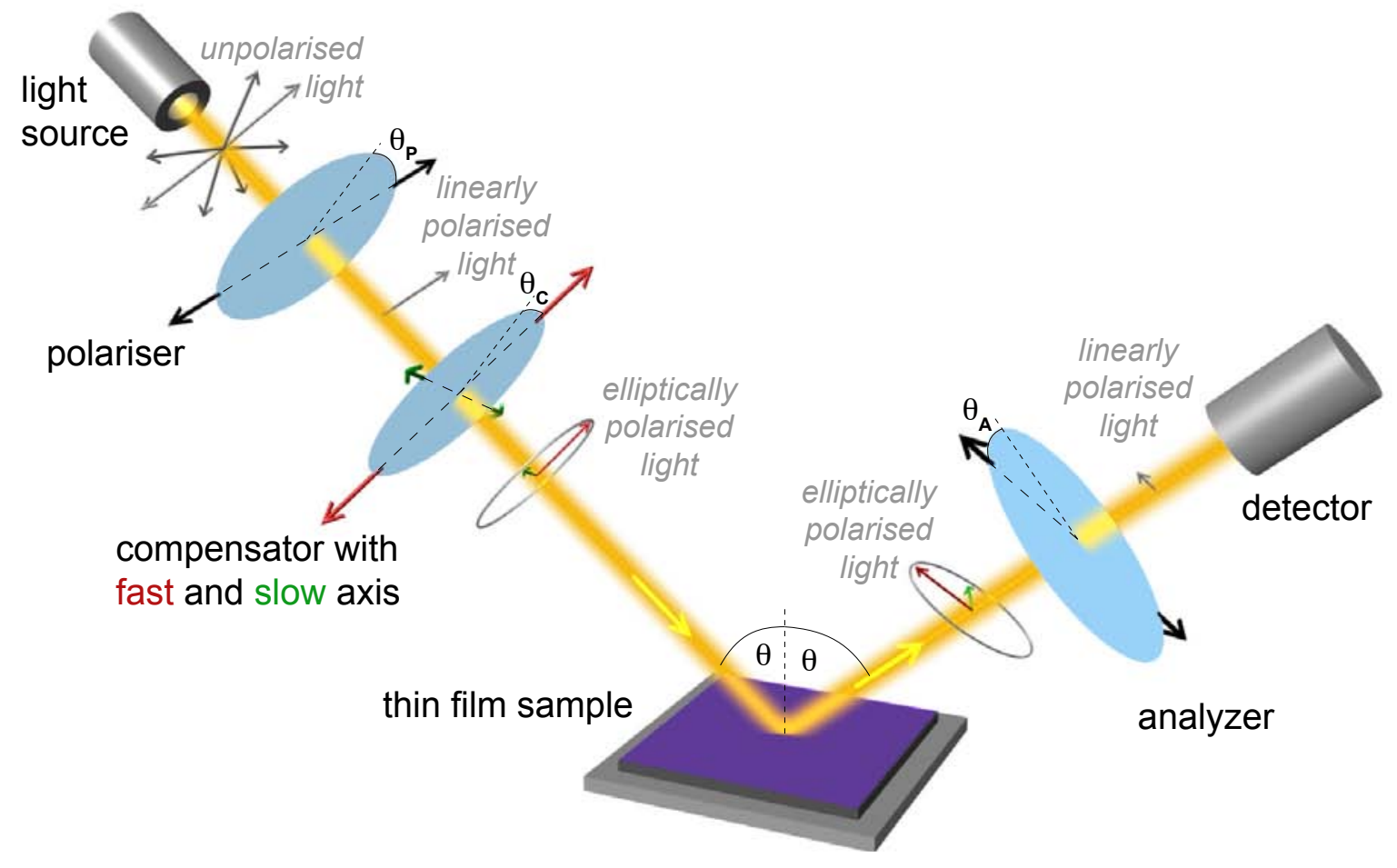

Figure 3.14: Schematic of the ellipsometer. 
and is reflected off the sample. The reflected light then passes through a second polariser (the analyser) before being detected (Fig. 3.14). The detector is formed of a CCD array, allowing for the local properties of the sample to be investigated. This technique is know as imaging ellipsometry.

The effect of the sample and each of the components in this set-up on the polarisation of the light can be expressed in the form of Jones matrices [70] giving

$$
\begin{gathered}
\left(\begin{array}{c}
E_{\mathrm{d} \|} \\
E_{\mathrm{d} \perp}
\end{array}\right)=R\left(\theta_{\mathrm{A}}\right)\left(\begin{array}{ll}
1 & 0 \\
0 & 0
\end{array}\right) R\left(-\theta_{\mathrm{A}}\right) \cdot\left(\begin{array}{cc}
\left|r_{\|}\right| \mathrm{e}^{\mathrm{i} \delta_{\|}} & 0 \\
0 & \left|r_{\perp}\right| \mathrm{e}^{\mathrm{i} \delta_{\perp}}
\end{array}\right) \cdot R\left(\theta_{\mathrm{C}}\right)\left(\begin{array}{ll}
1 & 0 \\
0 & \mathrm{i}
\end{array}\right) R\left(-\theta_{\mathrm{C}}\right) \cdot R\left(\theta_{\mathrm{P}}\right)\left(\begin{array}{ll}
1 & 0 \\
0 & 0
\end{array}\right) R\left(-\theta_{\mathrm{P}}\right) \cdot\left(\begin{array}{c}
E_{\mathrm{i} \|} \\
E_{\mathrm{i} \perp}
\end{array}\right) \\
\text { Analyser } \quad \text { Sample } \quad \text { Compensator Polariser }
\end{gathered}
$$

where $E_{\mathrm{d} \|}, E_{\mathrm{d} \perp}, E_{\mathrm{i} \|}$ and $E_{\mathrm{i} \perp}$, are the parallel and perpendicular field components of the detected and incident light, $r_{\|}=\left|r_{\|}\right| \mathrm{e}^{\mathrm{i} \delta_{\|}}$and $r_{\perp}=\left|r_{\perp}\right| \mathrm{e}^{\mathrm{i} \delta_{\perp}}$ are the Fresnel reflection coefficients of the sample, $\theta_{\mathrm{A}}, \theta_{\mathrm{C}}$ and $\theta_{\mathrm{P}}$, are the angles of the analyser, compensator and polariser with respect to the light incidence plane and $R\left( \pm \theta_{A, C, P}\right)$ are rotation matrices which are given by

$$
R\left( \pm \theta_{\mathrm{A}, \mathrm{C}, \mathrm{P}}\right)=\left(\begin{array}{cc}
\cos \theta_{\mathrm{A}, \mathrm{C}, \mathrm{P}} & \pm \sin \theta_{\mathrm{A}, \mathrm{C}, \mathrm{P}} \\
\mp \sin \theta_{\mathrm{A}, \mathrm{C}, \mathrm{P}} & \cos \theta_{\mathrm{A}, \mathrm{C}, \mathrm{P}}
\end{array}\right)
$$

$\Delta$ and $\Psi$ are the ellipsometric angles. These contain information about the amplitude ratio and the phase difference of parallel and perpendicularly polarised light upon reflection and are given by

$$
\begin{aligned}
& \tan \Psi=\frac{\left|r_{\|}\right|}{\left|r_{\perp}\right|}, \\
& \Delta=\delta_{\|}-\delta_{\perp} .
\end{aligned}
$$

The position of the polariser and compensator can be chosen so that the light reflected from the sample is linearly polarised and can be extinguished with the analyser. A minimum in the intensity is then detected at the CCD and $\Delta_{\exp }(\theta)$ and $\Psi_{\exp }(\theta)$ can be 
determined from the orientation of the polariser, analyser and compensator. Here, $\theta$ is the angle of light incidence.

The refractive index, the absorption coefficient and the film thickness cannot directly be deduced from $\Delta_{\exp }(\theta)$ and $\Psi_{\exp }(\theta)$. Instead, the ellipsometric angles have to be modelled assuming reasonable starting values for $n, k$ and $d$, giving $\Delta_{\mathrm{th}}(\theta)$ and $\Psi_{\mathrm{th}}(\theta)$. The modelling data is then fitted to the experimental values $\Delta_{\exp }(\theta)$ and $\Psi_{\exp }(\theta)$, allowing values of $n, k$ and $d$ for which the modelling data best matches the experimental results to be found.

Spectroscopic or angularly resolved ellipsometry measurements are widely used to determine refractive indices $n$, absorption coefficients $k$, and film thicknesses $d$, of flat layers made from a variety of non emissive materials. However, these measurements can be quite time-consuming. Alternatively, the thickness of thin films with known refractive indices and absorption coefficients deposited on glass or silicon can be determined by simple reflectivity measurements in a back-reflection geometry. In parallel, the reflectivity of the thin film is modelled as a function of film thickness using the algorithm described in Chapter 1. The film thickness is obtained by fitting the modelling results to the experimental data. 



\section{References}

[1] Resnick, P. \& Buck, W. Teflon ${ }^{\circledR}$ AF amorphous fluoropolymers. In Scheirs, J. (ed.) Modern fluoropolymers, 397-419 (John Wiley \& Sons Ltd, 2000).

[2] Bandrup, J., Immergut, E. H. \& Grulke, E. A. (eds.) Polymer handbook (John Wiley \& Sons, 1999), $4^{\text {th }}$ edn.

[3] Fletcher, R., Lidzey, D. \& Bradley, D. Spectral properties of resonant-cavity, polyfluorene light-emitting diodes. Appl. Phys. Lett. 77, 1262-1264 (2000).

[4] Herguth, P., Jiang, X., Liu, M. \& Alex, K. Highly efficient fluorene-and benzothiadiazole-based conjugated copolymers for polymer light-emitting diodes. Macromolecules 35, 6094-6100 (2002).

[5] Xia, R., Heliotis, G., Hou, Y. \& Bradley, D. Fluorene-based conjugated polymer optical gain media. Org. Electron. 4, 165-177 (2003).

[6] Xu, Q., Ouyang, J., Yang, Y., Ito, T. \& Kido, J. Ultrahigh efficiency green polymer light-emitting diodes by nanoscale interface modification. Appl. Phys. Lett. 83, 4695-4697 (2003).

[7] Hüttner, S. Donor-Acceptor Block Copolymers in Organic Electronics - Spectroscopy, Charge Transport, Morphology and Device Application. Ph.D. thesis, University of Bayreuth (2010).

[8] Sommer, M. Novel Semiconductor Block Copolymers for Organic Electronic Devices: Synthesis, Properties and Applications. Ph.D. thesis, University of Bayreuth (2009).

[9] Stevens, M., Silva, C., Russell, D. \& Friend, R. Exciton dissociation mechanisms in the polymeric semiconductors poly (9, 9-dioctylfluorene) and poly (9, 9-dioctylfluorene-co-benzothiadiazole). Phys. Rev. B 63, 165213 (2001). 
[10] Xia, R., Heliotis, G. \& Bradley, D. Fluorene-based polymer gain media for solidstate laser emission across the full visible spectrum. Appl. Phys. Lett. 82, 35993601 (2003).

[11] Pliskin, W. A. Comparison of properties of dielectric films deposited by various methods. J. Vac. Sci. Technol. 14, 1064-1081 (1977).

[12] Smit, M., Acket, G. \& van der Laan, C. $\mathrm{Al}_{2} \mathrm{O}_{3}$ films for integrated optics. Thin solid films 138, 171-181 (1986).

[13] Matero, R., Rahtu, A., Ritala, M., Leskelä, M. \& Sajavaara, T. Effect of water dose on the atomic layer deposition rate of oxide thin films. Thin Solid Films 368, $1-7(2000)$.

[14] Chen, K. et al. $\mathrm{SiO}_{2} / \mathrm{TiO}_{2}$ omnidirectional reflector and microcavity resonator via the sol-gel method. Appl. Phys. Lett. 75, 3805 (1999).

[15] Rausch, N. \& Burte, E. Thin $\mathrm{TiO}_{2}$ films prepared by low pressure chemical vapor deposition. J. Electrochem. Soc. 140, 145-149 (1993).

[16] Riihelä, D., Ritala, M., Matero, R. \& Leskelä, M. Introducing atomic layer epitaxy for the deposition of optical thin films. Thin Solid Films 289, 250-255 (1996).

[17] Wang, C. \& Ying, J. Sol-gel synthesis and hydrothermal processing of anatase and rutile titania nanocrystals. Chem. Mater 11, 3113-3120 (1999).

[18] Diebold, U. The surface science of titanium dioxide. Surf. Sci. Rep. 48, 53-229 (2003).

[19] Emslie, A., Bonner, F. \& Peck, L. Flow of a viscous liquid on a rotating disk. J. of Appl. Phys. 29, 858-862 (1958).

[20] Flack, W., Soong, D., Bell, A. \& Hess, D. A mathematical model for spin coating of polymer resists. J. Appl. Phys. 56, 1199 (1984).

[21] Meyerhofer, D. Characteristics of resist films produced by spinning. J. Appl. Phys. 49, 3993-3997 (1978). 
[22] Lawrence, C. The mechanics of spin coating of polymer films. Phys. Fluids 31, 2786 (1988).

[23] Crossland, E. J. Block Copolymer Patterning of Functional Materials. Ph.D. thesis, University of Cambridge (2008).

[24] Kolle, M., Zheng, B., Gibbons, N., Baumberg, J. \& Steiner, U. Stretch-tuneable dielectric mirrors and optical microcavities. Opt. Exp. 18, 4356-4364 (2010).

[25] Puurunen, R. L. Surface chemistry of atomic layer deposition: A case study for the trimethylaluminum/water process. J. Appl. Phys. 97, 121301 (2005).

[26] Leskelä, M. \& Ritala, M. Atomic layer deposition chemistry: Recent developments and future challenges. Angew. Chem. Int. Edit. 42, 5548-5554 (2003).

[27] Chen, P. et al. Atomic layer deposition to fine-tune the surface properties and diameters of fabricated nanopores. Nano Lett. 4, 1333-1337 (2004).

[28] Shin, H., Jeong, D.-K., Lee, J., Sung, M. \& Kim, J. Formation of $\mathrm{TiO}_{2}$ and $\mathrm{ZrO}_{2}$ nanotubes using atomic layer deposition with ultraprecise control of the wall thickness. Adv. Mater. 16, 1197-1200 (2004).

[29] King, J., Graugnard, E. \& Summers, C. $\mathrm{TiO}_{2}$ inverse opals fabricated using lowtemperature atomic layer deposition. Adv. Mater. 17, 1010-1013 (2005).

[30] King, J., Heineman, D., Graugnard, E. \& Summers, C. Atomic layer deposition in porous structures: 3D photonic crystals. Appl. Surf. Sci. 244, 511-516 (2005).

[31] Ras, R. et al. Hollow inorganic nanospheres and nanotubes with tunable wall thicknesses by atomic layer deposition on self-assembled polymeric templates. $A d v$. Mater. 19, 102-106 (2006).

[32] Graugnard, E. et al. Photonic band tuning in two-dimensional photonic crystal slab waveguides by atomic layer deposition. Appl. Phys. Lett. 89, 181108 (2006).

[33] Knez, M., Nielsch, K. \& Niinistö, L. Synthesis and surface engineering of complex nanostructures by atomic layer deposition. Adv. Mater 19, 3425-3438 (2007). 
[34] Leskelä, M. et al. Exploitation of atomic layer deposition for nanostructured materials. Mat. Sci. Eng. 27, 1504-1508 (2007).

[35] Kim, H., Lee, H. \& Maeng, W. Applications of atomic layer deposition to nanofabrication and emerging nanodevices. Thin Solid Films 517, 2563-2580 (2009).

[36] Aarik, J. et al. Characterization of titanium dioxide atomic layer growth from titanium ethoxide and water. Thin Solid Films 370, 163-172 (2000).

[37] Smith, R. et al. Chemical vapour deposition of the oxides of titanium, zirconium and hafnium for use as high-k materials in microelectronic devices. a carbon-free precursor for the synthesis of hafnium dioxide. Adv. Mater. Opt. Electr. 10, 105$114(2001)$.

[38] Jakschik, S. et al. Physical characterization of thin $\mathrm{ALD}-\mathrm{Al}_{2} \mathrm{O}_{3}$ films. Appl. Surf. Sci. 211, 352-359 (2003).

[39] Guziewicz, E. et al. Extremely low temperature growth of $\mathrm{ZnO}$ by atomic layer deposition. J. Appl. Phys. 103, 033515 (2008).

[40] Leskelä, M. \& Ritala, M. Atomic layer deposition (ALD): From precursors to thin film structures. Thin Solid Films 409, 138-146 (2002).

[41] Lim, B., Rahtu, A. \& Gordon, R. Atomic layer deposition of transition metals. Nature Materials 2, 749-754 (2003).

[42] Putkonen, M., Harjuoja, J., Sajavaara, T. \& Niinistö, L. Atomic layer deposition of polyimide thin films. J. Mater. Chem. 17, 664-669 (2007).

[43] Salmi, Puukilainen, E. \& Vehkamäki, M. Atomic layer deposition of $\mathrm{Ta}_{2} \mathrm{O}_{5} /$ polyimide nanolaminates. Chem. Vapor. Depos. 15, 221-226 (2009).

[44] Pilvi, T., Arstila, K., Leskela, M. \& Ritala, M. Novel ALD process for depositing $\mathrm{CaF}_{2}$ thin films. Chem. Mater 19, 3387-3392 (2007).

[45] Pilvi, T. et al. Study of a novel ALD process for depositing $\mathrm{MgF}_{2}$ thin films. $J$. Mater. Chem. 17, 5077-5083 (2007). 
[46] Niskanen, A., Hatanpää, T., Arstila, K., Leskelä, M. \& Ritala, M. Radicalenhanced atomic layer deposition of silver thin films using phosphine-adducted silver carboxylates. Chem. Vapor. Depos. 13, 408-413 (2007).

[47] Wilson, C., Grubbs, R. \& George, S. Nucleation and growth during $\mathrm{Al}_{2} \mathrm{O}_{3}$ atomic layer deposition on polymers. Chem. Mater 17, 5625-5634 (2005).

[48] Jeong, C., Lee, J. \& Joo, S. Plasma-assisted atomic layer growth of high-quality aluminum oxide thin films. Jpn. J. Appl. Phys. 40, 285-289 (2001).

[49] Jaehyoung, K., Yangdo, K. \& Hyeongtag, J. $\mathrm{ZrO}_{2}$ gate dielectric deposited by plasma-enhanced atomic layer deposition method. Jpn. J. Appl. Phys 41, 30433046 (2002).

[50] Ras, R., Sahramo, E., Malm, J., Raula, J. \& Karppinen, M. Blocking the lateral film growth at the nanoscale in area-selective atomic layer deposition. J. Am. Chem. Soc. 130, 11252-11253 (2008).

[51] Puurunen, R. Formation of metal oxide particles in atomic layer deposition during the chemisorption of metal chlorides: A review. Chem. Vapor. Depos. 11, 79-90 (2005).

[52] Elam, J., Routkevitch, D., Mardilovich, P. \& George, S. Conformal coating on ultrahigh-aspect-ratio nanopores of anodic alumina by atomic layer deposition. Chem. Mater. 15, 3507-3517 (2003).

[53] Haynes, C. \& Duyne, R. V. Nanosphere lithography: A versatile nanofabrication tool for studies of size-dependent nanoparticle optics. J. Phys. Chem. B 105, 5599-5611 (2001).

[54] Yang, S.-M., Jang, S. G., Choi, D.-G., Kim, S. \& Yu, H. K. Nanomachining by colloidal lithography. Small 2, 458-475 (2006).

[55] Wang, D. \& Mohwald, H. Rapid fabrication of binary colloidal crystals by stepwise spin-coating. Adv. Mater. 16, 244-247 (2004). 
[56] Jiang, P. \& McFarland, M. Large-scale fabrication of wafer-size colloidal crystals, macroporous polymers and nanocomposites by spin-coating. J. Am. Chem. Soc. 126, 13778 (2004).

[57] Chen, X. et al. Colloidal-crystal-assisted imprint for mesoscopic structured arrays and hierarchical patterns. Adv. Mater. 16, 1632-1636 (2004).

[58] Fudouzi, H. \& Xia, Y. Colloidal crystals with tunable colors and their use as photonic papers. Langmuir 19, 9653-9660 (2003).

[59] Zhang, X., Whitney, A., Zhao, J. \& Duyne, E. H. R. V. Advances in contemporary nanosphere lithographic technique. J. Nanosci. Nanotechnol. 6, 1920-1934 (2006).

[60] Scherer, M. R. Nanostructured Materials via Self-Assembled Templates. Master's thesis, University of Cambridge (2009).

[61] Denkov, N. et al. Mechanism of formation of two-dimensional crystals from latex particles on substrates. Langmuir 8, 3183-3190 (1992).

[62] Commonwealth Scientific Corporation. Ion beam etch rates. Bulletin \# 137-78.

[63] Williams, K., Gupta, K. \& Wasilik, M. Etch rates for micromachining processingpart II. J. Microelectromech. S. 12, 761-778 (2003).

[64] Chou, S., Krauss, P. \& Renstrom, P. Imprint of sub-25 nm vias and trenches in polymers. Appl Phys Lett. 67, 3114-3116 (1995).

[65] Chou, S., Krauss, P. \& Renstrom, P. Imprint lithography with 25-nanometer resolution. Science $\mathbf{2 7 2}, 85$ (1996).

[66] Wu, C., Shen, Y., Chuang, S. \& Wei, C. Anti-adhesive effects of diverse selfassembled monolayers in nanoimprint lithography. Sensor. Actuat. A - Phys. 139, 145-151 (2007).

[67] Barbero, D. R. et al. High resolution nanoimprinting with a robust and reusable polymer mold. Adv. Funct. Mater 17, 2419-2425 (2007). 
[68] Tompkins, H. \& McGahan, W. Spectroscopic Ellipsometry and Reflectometry - A User's Guide (John Wiley \& Sons, Inc., 1999).

[69] Tompkins, H. Handbook of Ellipsometry (William Andrew Inc., 2005).

[70] Azzam, R. \& Bashara, N. Ellipsometry and polarized light (Elsevier, 1987). 



\section{Chapter 4}

\section{Static and tuneable one-dimensional photonic structures}

Dielectric multilayer interference structures are widely applied in optics research and industry as low-loss reflectors, broadband mirrors, or filters. Commonly, such structures are called $1 \mathrm{D}$ photonic crystals due to the periodicity along the direction normal to the layer planes [1]. This chapter presents several approaches to create layered photonic structures made from organic or inorganic dielectric materials with static or tuneable optical performance.

\subsection{Static one-dimensional optical devices}

For many applications, dielectric multilayer interference structures are only required to display controlled reflection or transmission properties in a particular wavelength range without the need to provide optical tunability. The optical behaviour of static distributed Bragg reflectors (DBRs, but also called Bragg mirrors), multilayer resonance cavities (often termed Bragg filters) or chirped broadband mirrors that rely on a welldefined thickness variation throughout the multilayer stack can be adjusted to the application requirements by controlling the thickness of each of the dielectric layers within the multilayer.

Multilayer structures are often made from inorganic materials [2], involving vacuum deposition techniques that allow for very precise thickness control. The transmission characteristics of an inorganic Bragg filter made from titania and alumina in an Atomic Layer Deposition (ALD) process are shown in Figure 4.1. The filter consisting of an 

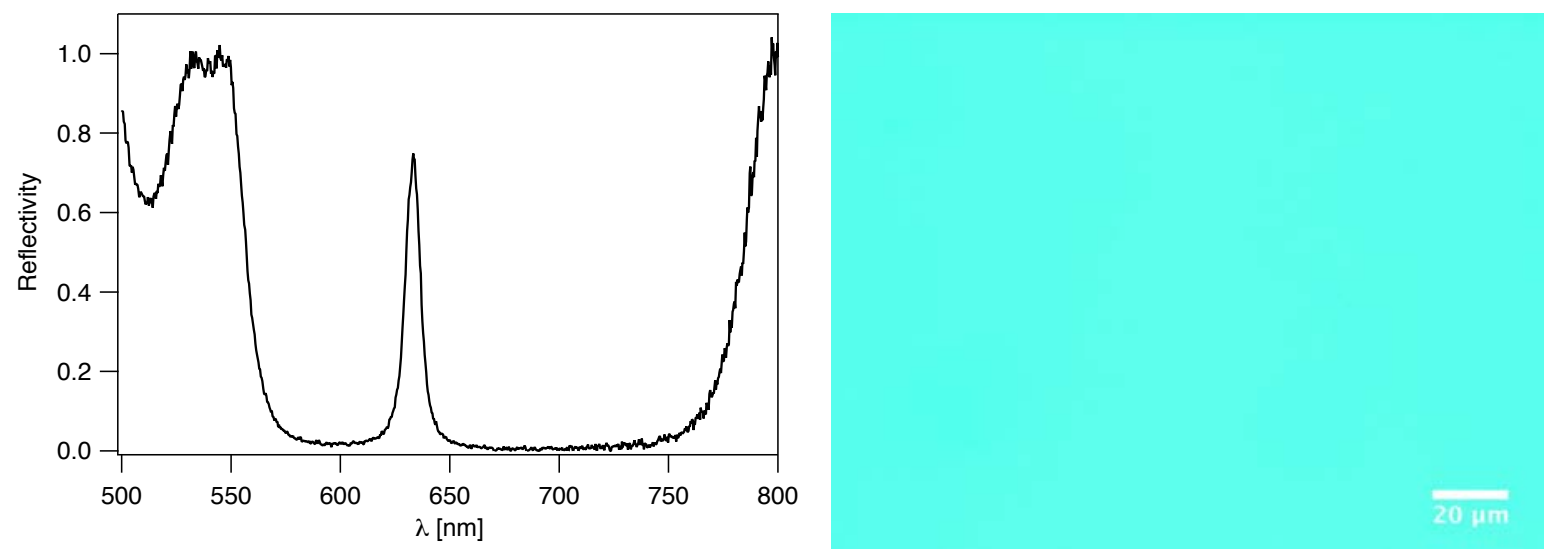

Figure 4.1: Inorganic Bragg filter. Transmission spectrum and transmission micrograph of an inorganic resonance cavity, consisting of an alumina cavity layer, enclosed by two titania-alumina quarter-wave DBRs. The pass-band lies at $633 \mathrm{~nm}$, with a bandwidth of $8 \mathrm{~nm}$, resulting in a quality factor, $\mathrm{Q} \approx 80$.

alumina cavity, enclosed by two Bragg reflectors of only nine layers each, has a passband at $633 \mathrm{~nm}$ with a bandwidth of $8 \mathrm{~nm}$, resulting in a quality factor of $~ 80$ and shows very good optical homogeneity over several square-centimetres. However, the production of such optical elements by ALD is rather time-consuming and not very cost-efficient. All-polymer based structures that can be produced with good thickness control $[3,4]$ offer a competitive alternative to inorganic multilayers. Polymer based optical elements have several advantages. Commonly available commodity polymers are usually very cheap. Polymer materials can be conveniently processed from solution or from the melt. Polymer processing techniques do not require high vacuum equipment, which makes them comparably cost- and time efficient. In this section, an approach for the production of static multilayer reflectors (Bragg mirrors) and resonance cavities (Bragg filters) is discussed.

\subsubsection{Organic Bragg reflectors}

The polymer materials used in the layer stacks have to fulfil a certain set of requirements depending on the purpose of the anticipated optical structure and the applied processing procedures. The aim in the context of this thesis was the creation of low-loss dielectric mirrors for various wavelength ranges which could be transferred onto other 
target surfaces by floating or which could be used in free-standing geometries.

Usually, in the choice of materials, a compromise has to be found between the processing requirements and the desired optical performance. Polystyrene (PS) and Teflon ${ }^{\circledR} \mathrm{AF}$ have been chosen as suitable materials for multilayer structures due to their good optical properties and their relative ease of processing. PS has a refractive index of $n_{\mathrm{PS}}=1.586$ and negligible absorption. It is very well established in the production of thin, smooth films, ranging from $\sim 10 \mathrm{~nm}$ to several $\mu \mathrm{m}$ in thickness, by spin-coating from solutions in toluene. Teflon ${ }^{\circledR}$ AF 1600, a fluorinated copolymer from DuPont, was chosen for its low refractive index of $n_{\mathrm{TAF}}=1.31$. With an effective refractive index contrast of $\hat{n}=\frac{n_{\mathrm{PS}}}{n_{\mathrm{TAF}}}=1.21$, this material combination allows fabrication of multilayers with theoretically predicted reflectances of $\sim 90 \%$ from as little as 15 alternating layers (Chapter 1). FC-75 from 3M was used as solvent for Teflon ${ }^{\circledR} \mathrm{AF}$ and layers with thicknesses of $30 \mathrm{~nm}-1 \mu \mathrm{m}$ were made by spin-coating. Typical solution concentrations ranged from $1.5 \%$ to $2.5 \%$ of polystyrene in toluene and $1.25-1.75 \%$ of Teflon ${ }^{\circledR} \mathrm{AF}$ in FC-75. More information about the polymer materials can be found in Chapter 3 .

FC-75 and toluene are an orthogonal solvent system for Teflon ${ }^{\circledR}$ AF and PS. Consequently, sequential deposition of polystyrene and Teflon ${ }^{\circledR} \mathrm{AF}$ layers by spin coating is in principle possible, without dissolving the immediately underlying layer. While the deposition of Teflon ${ }^{\circledR}$ AF on PS by spin coating works, deposition of polystyrene on Teflon ${ }^{\circledR}$ AF films can not be realised with the same ease. The inherent hydrophobicity of the Teflon ${ }^{\circledR}$ AF renders its processing rather difficult. Deposition of a PS layer on top of a Teflon ${ }^{\circledR}$ AF layer by spin coating could not be achieved without pre-treatment of the Teflon ${ }^{\circledR}$ AF surface, as the PS solution does not sufficiently wet the Teflon ${ }^{\circledR} \mathrm{AF}$ film and flies off the surface during the spinning process. However, a very short exposure of the Teflon ${ }^{\circledR}$ AF film to an oxygen plasma increases toluene wettability, allowing for the deposition of a polystyrene film on Teflon ${ }^{\circledR}$ AF substrates. Typical exposure times in the oxygen plasma were less than $2 \mathrm{~s}$ at a pressure of $\sim 3 \mathrm{mbar}$ and a nominal power of $100 \mathrm{~W}$. Optical micrographs and photographs of three different prototype multilayer stacks, containing 15 alternating PS and Teflon ${ }^{\circledR}$ AF layers, with different layer thicknesses are shown in Figure 4.2. The samples were deposited on glass sub- 


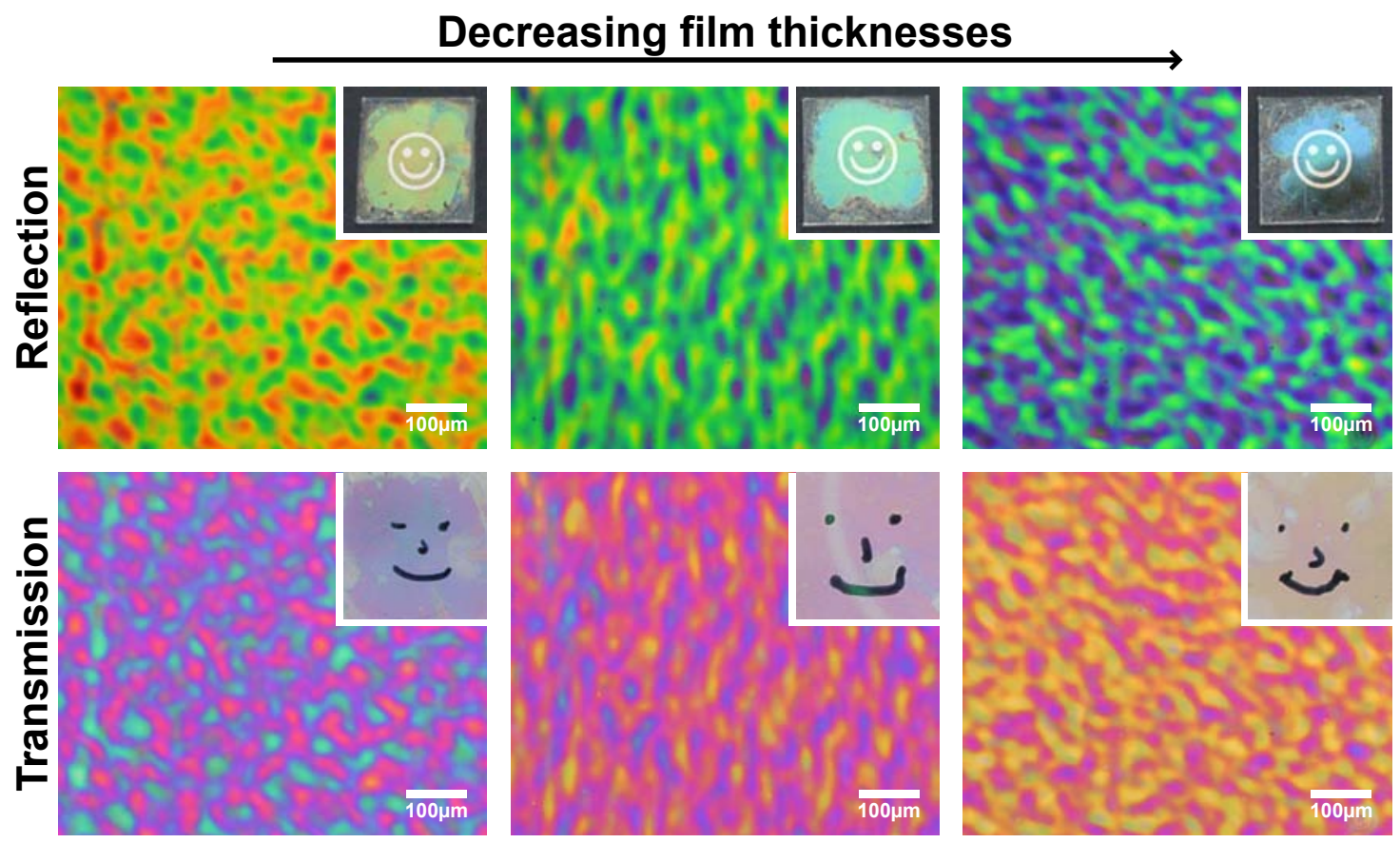

Figure 4.2: All-polymer multilayer films. Reflection and transmission of prototype multilayer films imaged by microscopy and photography, Samples were spin-cast from polystyrene and Teflon ${ }^{\circledR}$ AF solutions at $2500 \mathrm{rpm}, 3000 \mathrm{rpm}, 3500 \mathrm{rpm}$ (from left to right). Film thicknesses decrease from left to right but do not fulfil the ideal multilayer interference conditions. The films are semi-transparent as can be seen by the motifs under the multilayer samples in the insets of the microscope images.

strates and the top and bottom layer of each stack was made from polystyrene. The film thicknesses of these samples were not tuned to match the conditions for ideal multilayer reflectance (Section 1.3.1). A non-negligible optical anisotropy of the films becomes apparent in the pronounced colour fluctuations, seen in the micrographs. This anisotropy results from an inherent waviness that arises when Teflon ${ }^{\circledR}$ AF films are deposited onto the polystyrene layers. The amplitude of the wave patterns is of the order of $25 \mathrm{~nm}$, as determined by Atomic Force Microscopy (AFM). At this point, the origin of these undulations is not entirely clear. Hydrodynamic instabilities, polymer-solvent demixing, or the onset of a dewetting instability, frustrated by the freezing-in of the structure in the glassy polymer upon solvent evaporation could lead to similar fluctuations. Attempts to speed up the solvent evaporation by heating the polymer solution to $60^{\circ} \mathrm{C}-70^{\circ} \mathrm{C}$ prior to spin-coating did not result in a significant reduction of the wave pattern amplitude. 

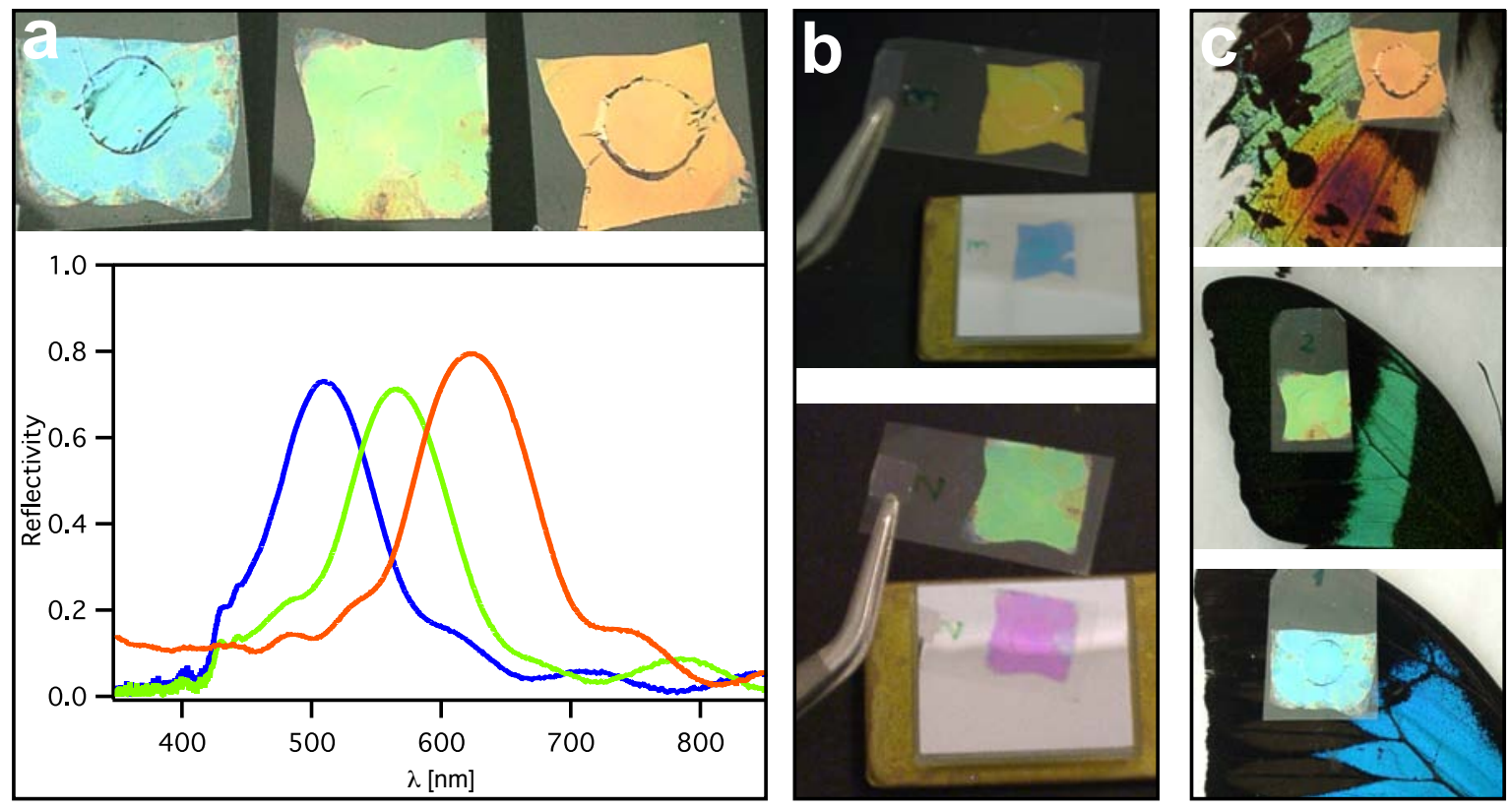

Figure 4.3: Adjusting multilayer film colour. a) Three film stack with 15 layers each, spin-cast at different rotation speeds to adjust the reflection colour. Film thickness in the stacks increase from left to right. Reflectivity spectra show the shift of the reflectance peak to higher wavelengths with increasing layer thicknesses. b) A mirror can be used to simultaneously image the complementary reflection and transmission colours. c) Comparison of the multilayer's optical performance to their natural role models.

This variation in the Teflon ${ }^{\circledR}$ AF film thicknesses obviously imposes severe constraints on the performance of the produced optical elements. However, Bragg reflectors and filters manufactured with the amorphous Teflon ${ }^{\circledR}$ AF showed excellent local and satisfactory global optical properties, which are discussed below.

All-polymer Bragg reflectors with the stop-band positioned in different spectral ranges are presented in Figure $4.3 \mathrm{a}$. The colour of the films can be tuned through the whole visible spectral range by adjusting the layer thicknesses. The three films were spincast at $3000 \mathrm{rpm}$ (blue), $2500 \mathrm{rpm}$ (green) and $2000 \mathrm{rpm}$ (orange) and consequently the layer thicknesses increase from the blue to the orange film. The film thicknesses determined from fitting the reflection spectra were $d_{\mathrm{PS}} \approx 112 \mathrm{~nm}, d_{\mathrm{TAF}} \approx 57 \mathrm{~nm}$ for the blue reflector, $d_{\mathrm{PS}} \approx 118 \mathrm{~nm}, d_{\mathrm{TAF}} \approx 71 \mathrm{~nm}$ for the green multilayer and $d_{\mathrm{PS}} \approx 125 \mathrm{~nm}$, $d_{\mathrm{TAF}} \approx 84 \mathrm{~nm}$ for the orange film. However, these thickness can only be considered as approximations of average values. The TEM cross-section of the blue multilayer shows 


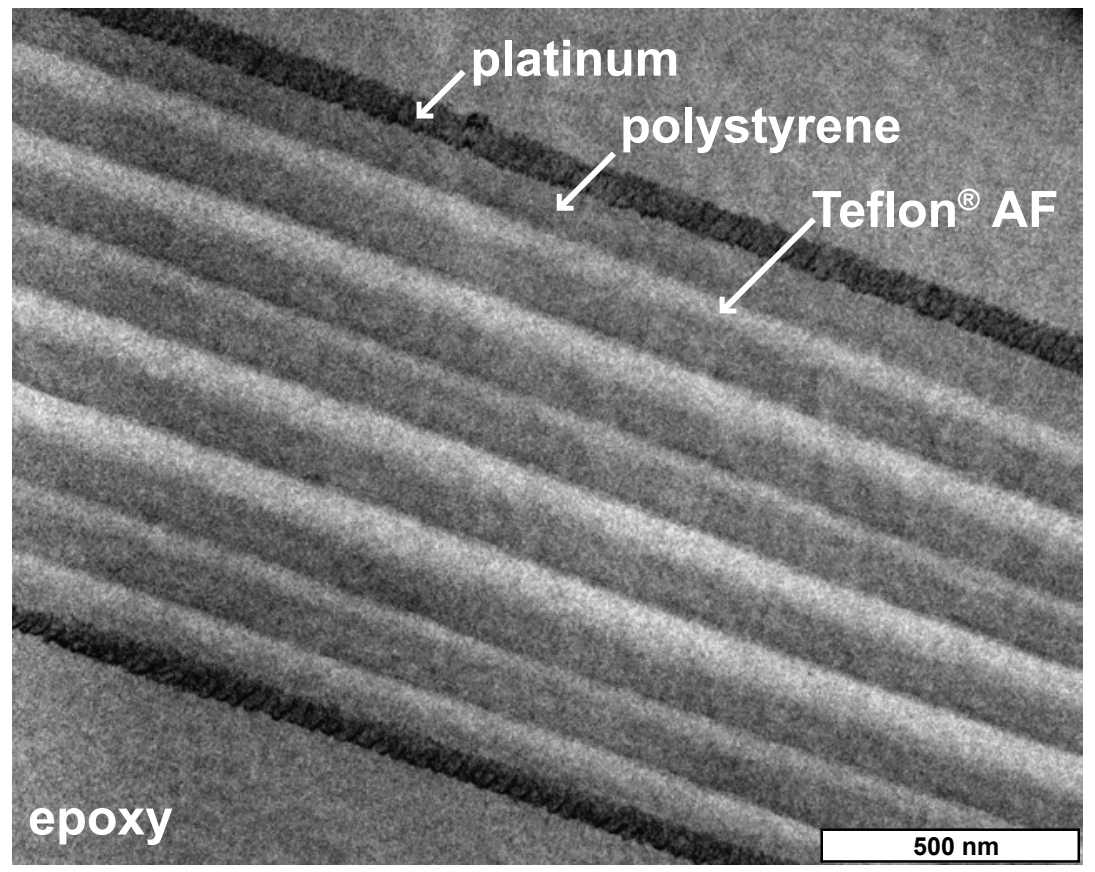

Figure 4.4: Cross-section of a multilayer film. The transmission electron micrograph visualises the individual polystyrene and Teflon ${ }^{\circledR}$ AF layers in the blue multilayer shown in Fig. 4.3. A thin layer of platinum was deposited on either side of the multilayer to facilitate the image acquisition.

that there is a significant variation of film thicknesses across the stack (Fig. 4.4). These simple non-ideal Bragg mirrors perform well as colour filters which, depending on their layer thicknesses, separate a specific colour by reflection from its complementary hue that is transmitted (Fig. $4.3 \mathrm{~b}$ ). In reflected intensity, the films are comparable to their natural role models (Fig. $4.3 \mathrm{c}$ ), provided they are observed in specular reflection.

To produce multilayers with controlled homogeneous layer thicknesses throughout the stack, the dependence of the film thickness on the spin coating parameters was determined for the respective polymer solutions. The thickness of the final polymer film as a function of angular velocity $\omega$ is given by the relation

$$
h=\left(\frac{3 \nu_{0} e_{0} c_{0}^{3}}{2\left(1-c_{0}\right)}\right)^{\frac{1}{3}} \cdot \omega^{-\frac{1}{2}},
$$

where $\omega$ is the angular velocity, $c_{0}$ the concentration of the polymer solution, $\nu_{0}$ its viscosity and $e_{0}$ the evaporation constant [5]. Without knowing the exact values of $\nu_{0}$ and $e_{0}$ this relation can be used to fit the experimentally obtained (thickness, spin speed)-data 

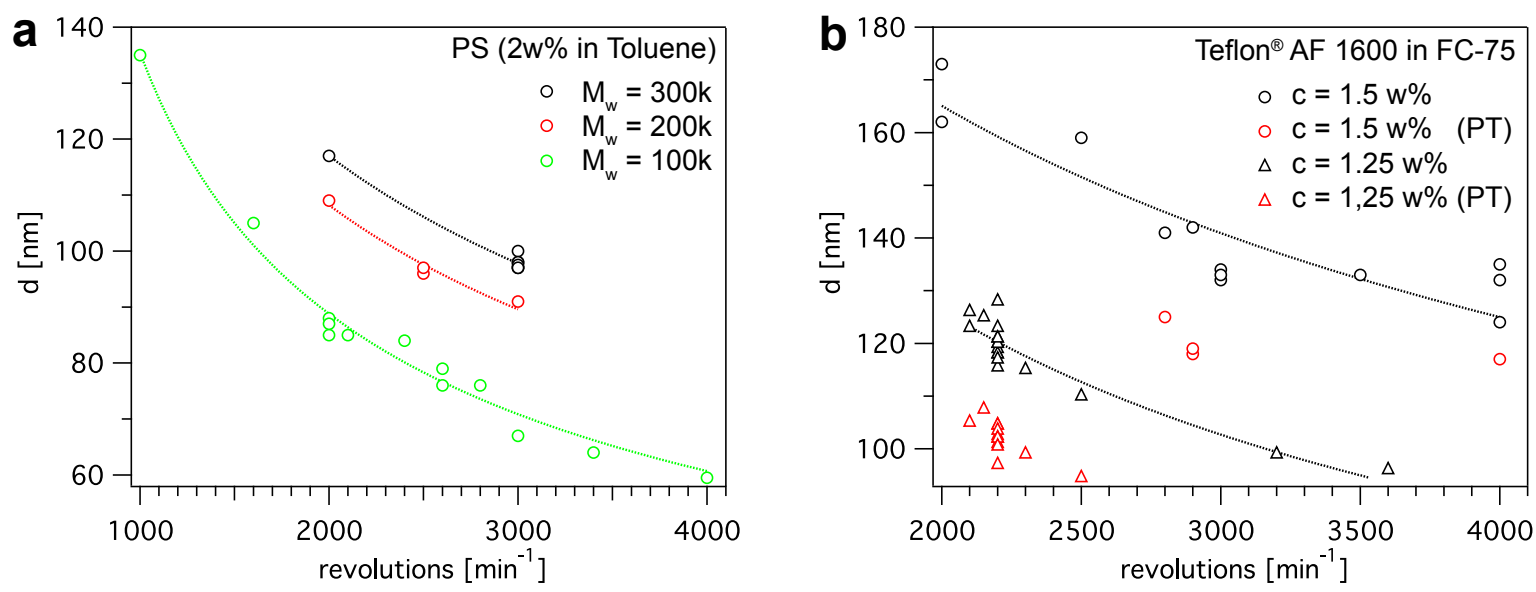

Figure 4.5: Film thickness as a function of spin speed. a) Thickness of polystyrene films on glass substrates as a function of spin speed for $2 \mathrm{w} \%$ solution concentration and different molecular weights. b) Thickness of Teflon ${ }^{\circledR}$ AF films as a function of spin speed for $1.25 \mathrm{w} \%$ and $1.5 \mathrm{w} \%$ solution concentration before plasma treatment (PT). The thicknesses of films after plasma-etching are shown with the red symbols. The lines in both graphs represent fits of the data based on Equation 4.1.

and to predict the film thickness as a function of angular velocity and solution concentration, provided the experimental conditions, such as temperature and humidity, do not drastically change. Results for the spinning of $2 \mathrm{wt} \%$-solutions polystyrene with molecular weights $M_{\mathrm{w}}$ of $100,000 \mathrm{~g} / \mathrm{mol}, 200,000 \mathrm{~g} / \mathrm{mol}$ and $300,000 \mathrm{~g} / \mathrm{mol}$ are shown in Figure 4.5 a. The thickness dependence of Teflon ${ }^{\circledR}$ AF films on spin speed is plotted in Figure $4.5 \mathrm{~b}$ for $1.25 \mathrm{wt} \%$ and $1.5 \mathrm{wt} \%$ concentrations. Furthermore the thickness of the Teflon ${ }^{\circledR}$ AF films after oxygen plasma treatment $(\mathrm{PT})$ at 0.3 mbar for $0.8 \mathrm{~s}$ is displayed. The thickness of the films decreases by $18 \mathrm{~nm} \pm 7 \mathrm{~nm}$ due to the plasma treatment.

With better control over the individual layer thicknesses, the stop-band of Bragg mirrors can be tailored to individual specifications and planar resonance cavities with controlled resonance wavelength can be fabricated.

\subsubsection{Organic resonance cavities}

Many optical bandpass filters are realised in the form of dielectric, planar resonance cavities, often called Bragg filters. In addition, resonance cavities can influence the luminescence characteristics of a dye that is incorporated into the cavity layer, if the 
pass-band of the cavity lies in the spectral emission range of the dye [6, 7].

To test the suitability of the PS-Teflon ${ }^{\circledR}$ AF multilayer structures for bandpass filters with an optically active dye component, the cavity layer in a resonance cavity was produced from Poly (perylenebisimideacrylate) [8, 9] which absorbs light at wavelengths from $400 \mathrm{~nm}$ to $550 \mathrm{~nm}$ and shows emission in the range of $550 \mathrm{~nm}$ to $750 \mathrm{~nm}$. The dye material can be spin-cast from solutions in chloroform.

A schematic of the produced dye cavity with two 15-layer Bragg reflectors is shown in Fig. 4.6 a. Displayed below, are the experimentally measured reflection spectra and the theoretical fits measured after the deposition of each polystyrene layer during the production of the Bragg reflectors, starting with three layers finishing at fifteen (Fig. 4.6 b). The reflectivity and the optical appearance of the final Bragg reflectors are shown in Fig. $4.6 \mathrm{c}$. The black circle represents the sample area from which the spectra were acquired. A passive resonance cavity can simply be made by stacking two of the 15-layered Bragg reflectors (Fig. 4.6 d). In this configuration quality factors of $\sim 60$ were achieved. The dye-loaded cavity is made by spinning the dye cavity layer onto a Bragg reflector and floating the second Bragg reflector on top. The locally measured emission behaviour of the cavity that is excited at $405 \mathrm{~nm}$ can be seen in the top graph in Figure 4.6 e (black). For comparison the emission spectra of a simple unconfined dye layer is shown (red). Below the reflectivity of the cavity and its optical appearance are shown. Averaged over an area of $\sim 500 \mu^{2}$, the highest locally measured quality factor was $Q=75$. The confinement leads to a 3.7-fold enhancement of the dye emission in the pass-band of the cavity. Globally however, the performance of these cavity structures is limited by the waviness of the DBR structures, as discussed previously.

The global emission behaviour of a dye in a PS-Teflon ${ }^{\circledR}$ AF resonance cavity was studied, using poly (9,9 '-dioctylfluorene-co-benzothiadiazole) with a molecular weight of $5000-8000 \mathrm{~g} / \mathrm{mol}$ (F8BT), a dye, that can be processed by spin-coating from a pxylene solution. The F8BT was excited with $460 \mathrm{~nm}$-laser light directed at an angle onto the cavity via an optical fibre and the emitted light was collected with another fibre connected to a USB2000 Ocean Optics spectroscope. A schematic of the setup is 

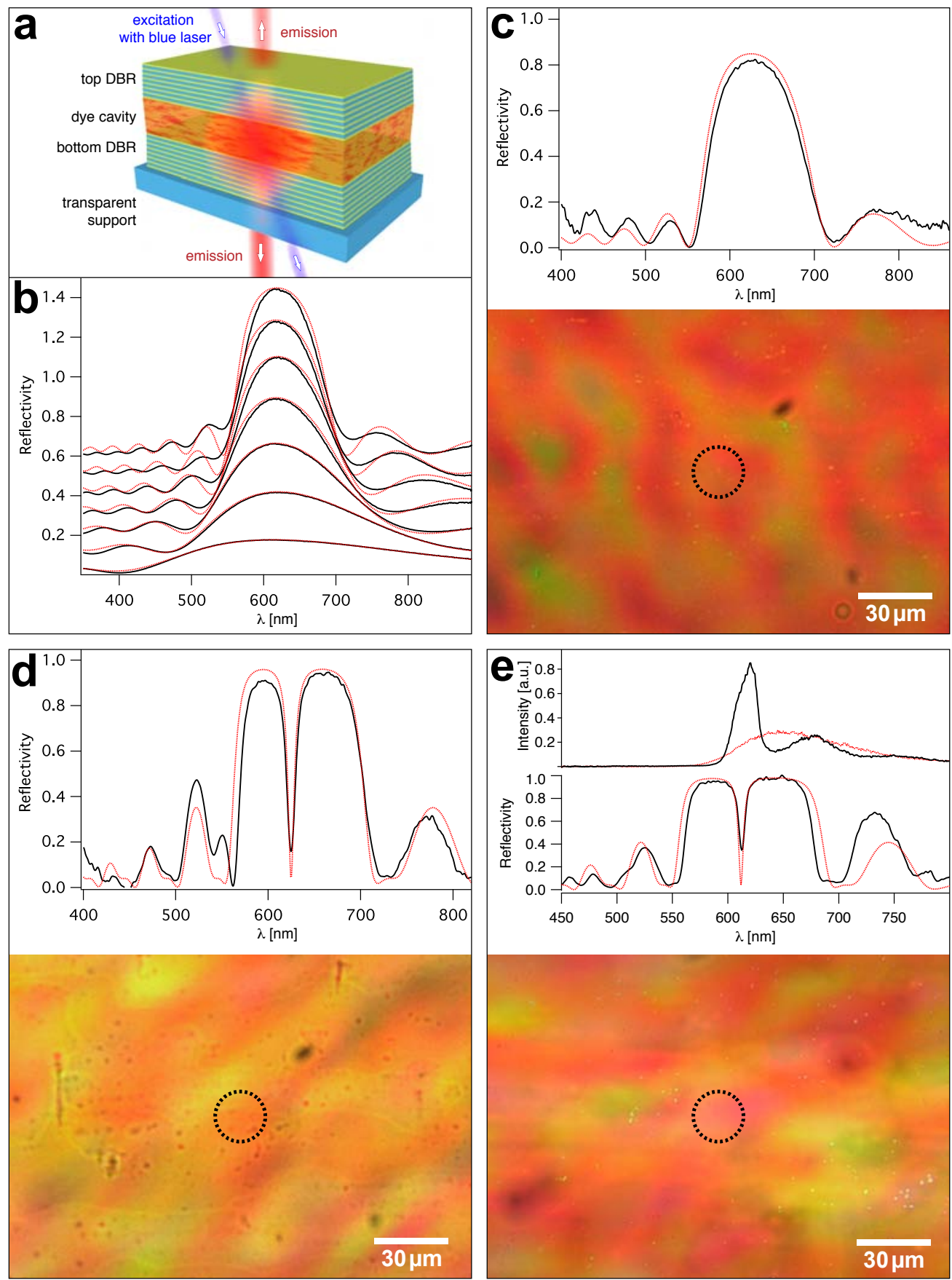

Figure 4.6: Construction of a dye-loaded resonance cavity. a) Schematic of the cavity assembly with two DBRs and a perylene bisimide dye-loaded cavity layer. b) Reflectance of the DBRs as a function of the number of layers in the stack. For clarity, the spectra are offset in steps of 0.1. c) Reflectivity curve and microscope image of one of the 15-layered DBRs. d) Reflectance and local optical appearance of a microcavity, made by stacking two 15-layered DBRs on top of each other. e) The top graph shows the emission behaviour of an optically excited, $185 \mathrm{~nm}$ thick dye cavity enclosed between two 15-layered DBRs (black) and the emission of an unconfined dye layer (red). Below, the experimentally obtained (black) and modelled (red) reflectance curves are plotted and a micrograph of the dye cavity surface is displayed. The dotted circles in the micrographs show the area on the samples from which the respective spectra were acquired. 

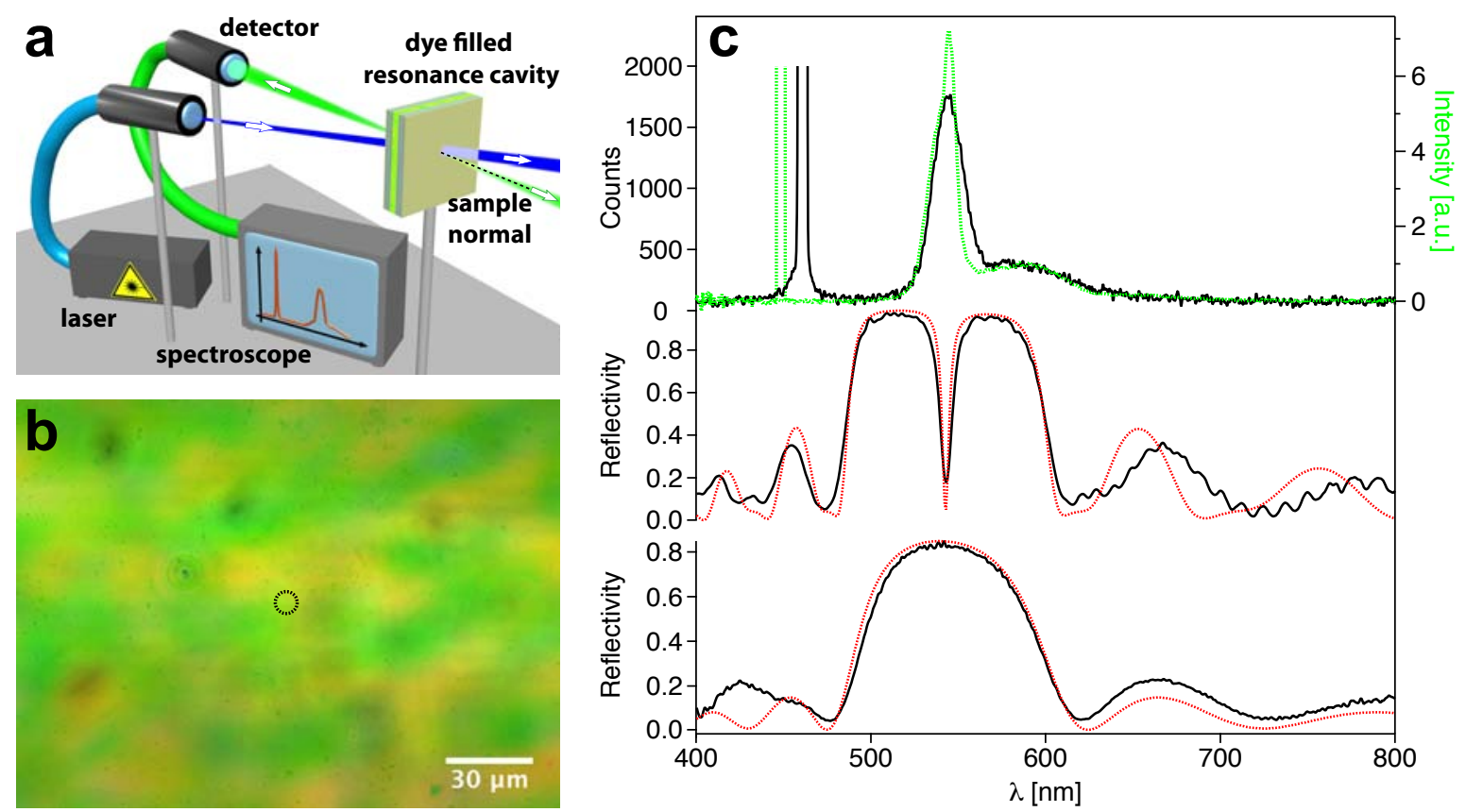

Figure 4.7: Excitation of a F8BT resonance cavity. a) Schematic of the set-up used to measure the excitation of the polymer dye in the resonance cavity. b) Micrograph of the cavity surface in reflection. The small circle indicates the area, from which the reflection spectrum of the unexcited dye filled cavity (shown in c) was acquired. c) The top spectrum shows the emission of the dye in the resonance cavity excited by a laser at $460 \mathrm{~nm}$, causing cavity-enhanced emission at $543 \mathrm{~nm}$. Local measurements taken from the spot shown in (b) are drawn in green, while measurements taken from a spot of several millimetres in diameter are displayed in the black line. The second graph contains the reflectivity spectrum of the unexcited resonance cavity and below the reflectance curve of one of the cavity DBRs is shown. Modelling data is plotted in red.

shown in Figure 4.7 a. The surface of the cavity structure is displayed in Figure $4.7 \mathrm{~b}$. The emission spectra and the reflectivity of the F8BT cavity are shown in Figure $4.7 \mathrm{c}$ together with the reflectivity of one of the cavity DBRs. The emission of the F8BT dye in the cavity with quality factor of $\sim 60$ is enhanced by more than one order of magnitude. F8BT is electroluminescent $[6,10,11]$. Work in progress includes the realisation of a working LED from dye-loaded planar PS-Teflon ${ }^{\circledR}$ AF-cavity structures by using thin, flexible, gold electrodes $[12,13]$ or a conducting polymer [14-16] of appropriate thickness on both sides of the cavity layer.

In summary, layered structures, made from polystyrene and Teflon ${ }^{\circledR} \mathrm{AF}$, could be promising alternatives to inorganic reflectors, filters or LEDs, provided more work is 
devoted to the control and ultimately suppression of the waviness in the Teflon layers. However, the necessity of a plasma-etching step considerably complicates the manufacturing process, loosing the advantage of simple and cheap processing that many polymer based systems usually offer over inorganic devices. Furthermore, the spin coating conditions have to be controlled extremely well, to minimise thickness variations across the multilayer. The probability of introducing defects into the multilayer raises with the number of spin coating steps. Thickness variations and defects ultimately limit the performance of the layered optical devices. The big advantage of the combination of polystyrene with the amorphous Teflon ${ }^{\circledR} \mathrm{AF}$ lies in the large refractive index contrast that can be achieved compared to combinations of other polymers. However, layered structures, involving different polymers can offer other advantages, such a tuneability or ease of processing.

In the following, a route to tuneable, organic, layered optical devices is discussed. This approach involves an alternative sample preparation technique, which addresses some of the fabrication issues mentioned above. The successful manufacture and characterisation of stretch-tuneable dielectric Bragg mirrors and resonance cavities was made possible by the excellent experimental work of Bo Zheng, Part III student in the Thin Films and Interfaces group in 2009.

\subsection{Stretch-tuneable dielectric mirrors and optical microcavities}

Periodic multilayers of transparent dielectrics are among the most common optical elements [17-19], used to produce high reflectivity Distributed Bragg Reflectors [20], broadband beamsplitters, optical filters, and optical microcavities. The latter are important for light emission in vertical cavity semiconductor lasers [21] and resonant cavity light emitting diodes [7]. While the manufacture of polymeric DBRs has been reported [3, 20, 22-24], the greatest drawback of multilayer optical components is their lack of tuneable performance due to their composition from rigid layers [25-29]. Here 
we demonstrate fabrication of entirely elastomeric multilayer dielectric stacks, which can be stretched by over $60 \%$ to achieve rapid, reversible wavelength tuning.

Typical tuning schemes for multilayer optical components use thermal expansion, which limits the accessible wavelength ranges [30], or solvent-swelled expansion, which predominantly acts on only one of the layer types, degrading the optical performance [31]. Promising recent block copolymer based multilayer mirrors operate only in liquid environments, thus producing significant additional challenges [32]. All of these mechanisms are slow and limited by solvent or thermal diffusion into the layer stacks. Conversely, compression of multilayer stacks produces only limited wavelength tuning [33, 34], while high reflectivity $3 \mathrm{D}$ elastomeric photonic crystals composed of spheres cannot yet be simply fabricated on large scales [35-37]. Our technique, on the other hand, relies on the direct deposition of only a single polymer bi-layer which is sequentially stacked to form high quality DBRs and microcavities. By using two polymer rubbers, the resulting optical structures are made highly flexible. Such thin flexible mirrors and cavities can be assembled into unusual configurations, are easily scalable to large-scale manufacture, and promise high quality factor performance without compromising tuning.

\subsubsection{Sample preparation and experimental setup}

Tuneable DBRs (often called 1D photonic crystals) are constructed from alternating layers of high and low refractive index materials, where for each layer in the stack

$$
\bar{\lambda}=4 d_{i} \sqrt{n_{i}^{2}-\sin ^{2} \theta}
$$

with $\bar{\lambda}$ being the central reflection stopband wavelength, $\theta$ is the angle of light incidence and $d_{i}, n_{i}$ are the thickness and refractive index of the $i$-th layer (see Section 1.3.1). The multilayer structures here are manufactured from two commodity elastomers, a silicone rubber, polymethylsiloxane (PDMS, Dow Corning - Sylgard 184) and an elastic tri-block copolymer, which consists of polyisoprene containing a $22 \mathrm{wt} \%$ minority polystyrene phase (PSPI, Sigma Aldrich). Both materials are transparent. The Pois- 
son ratio, a measure for the compression of an object perpendicular to the stretching direction, is 0.5 for PDMS and PSPI as for many rubbers. Consequently, applying an in-plane deformation to a PDMS-PSPI multilayer results in a proportional change in layer thickness. The refractive indices of thin PDMS and PSPI films are measured by ellipsometry to give $n_{\mathrm{PDMS}}=1.41 \pm 0.02$ and $n_{\mathrm{PSPI}}=1.54 \pm 0.02$.

The fabrication of the stretch-tuneable optical elements is shown in Fig. 4.8. PDMS is dissolved in heptane and spun onto a silicon substrate. Annealing in an oven at $120^{\circ} \mathrm{C}$ for one hour leads to cross-linking of the PDMS which makes it resistant to toluene, the solvent used for the PSPI copolymer. PSPI is subsequently spun onto the PDMS layer. The resulting layer thicknesses (determined by atomic force microscopy (AFM)

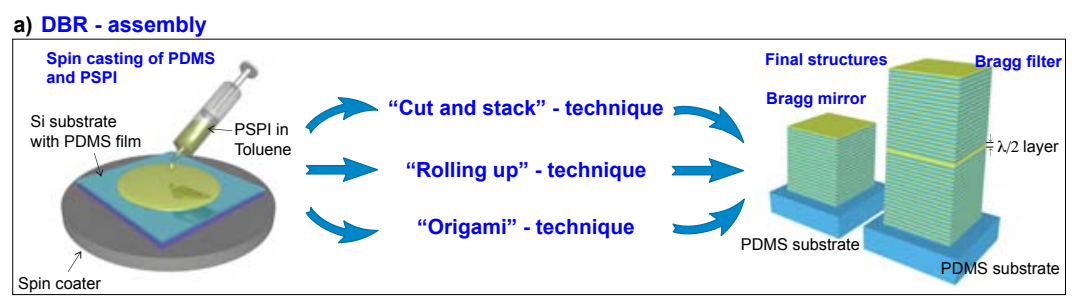

b) "Cut and stack" - technique

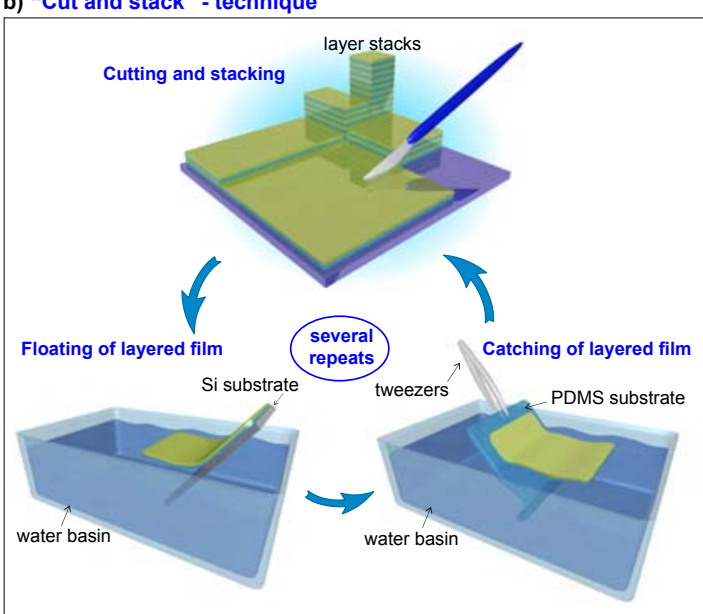

c) "Rolling up" - technique

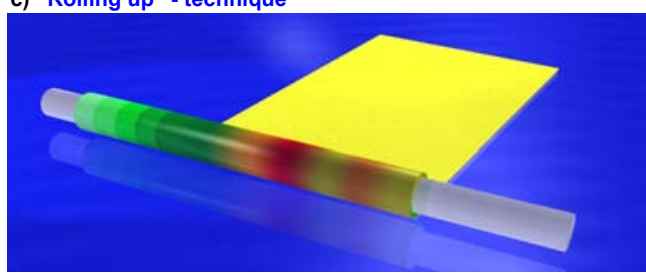

d) "Origami" - technique

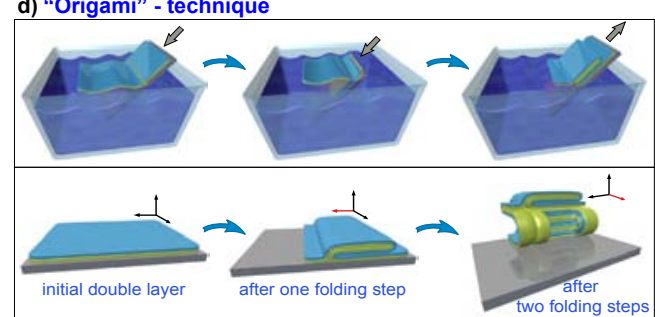

Figure 4.8: Schematic DBR assembly: a) Initial double layer produced by spin-coating a PDMS film followed by thermal annealing and spin-coating of a PSPI film on top to produce DBRs via three different routes. b) "Cut and stack" technique: repeated cutting and floating followed by the stacking of layered films on top of each other. c) "Roll-up" technique: double layer floated onto a water surface is rolled up onto a flexible transparent rod. d) "Origami" technique: based on three-step folding cycle leading to a $z$-fold. Lower box shows the initial bi-layer, and stacks after one and two folding cycles. Red arrows indicate the folding direction. 
and ellipsometry) of $d_{\mathrm{PDMS}}=165 \pm 10 \mathrm{~nm}$ and $d_{\mathrm{PSPI}}=295 \pm 10 \mathrm{~nm}$ are optimised to maximise the DBR reflectivity in the red part of the visible spectrum. The microphase separation of the polystyrene component in the PSPI leads to nanoscopic glassy minority domains in a polyisoprene matrix, which act as cross-links, rendering the curing of the polyisoprene rubber unnecessary.

Assembly into multilayer stacks can proceed via several routes. In the simplest process, the double layer is subsequently cut into small sections of $5 \mathrm{~mm} \times 10 \mathrm{~mm}$ on the silicon substrate. Pieces of double layer are released from the substrate by floating them onto a water surface and they are sequentially overlaid onto a PDMS rubber slab to form a multilayer. In this fashion a DBR consisting of 20 layers is assembled. In addition, a resonant multilayer microcavity consisting of two multilayer stacks enclosing a $\lambda / 2$ PSPI cavity layer is realized. The film thicknesses in the resonant microcavity are $d_{\mathrm{PDMS}} \approx 150 \mathrm{~nm}, d_{\mathrm{PSPI}} \approx 250 \mathrm{~nm}$ and $d_{\mathrm{PSPI} \text { cavity }} \approx 295 \mathrm{~nm}$.

This assembly procedure is time-consuming as the number of layers scales linearly with the number of floating steps. Furthermore, the likelihood of introducing defects into the structure increases with every floating step. In order to minimise the number of production steps three other highly-scaleable, less defect-prone techniques were also developed.

\section{“Cut and stack" technique}

An improved variant of stack manufacture consists of cutting an initial stack of $i$ layers in half and superposing one part onto the other to form a $2 i$-layer stack (Fig. $4.8 \mathrm{~b}$ ). Starting with an initial double layer this repeated 'cut and stack' procedure leads to a stack of 128 layers in only six steps. More generally the number of layers $l_{c}$ in a DBR stack varies as

$$
l_{c}=2^{c+1}
$$

with $c$ being the number of cut-and-stack operations. 


\section{“Roll-up” technique}

This method proceeds from a double-layer film, with the appropriate layer thicknesses $d_{1}, d_{2}$ (see Eq.4.2), that is floated onto a water surface. Subsequently, the floating bi-layer is rolled up onto a transparent, stretchable, cylindrical or square rod made from PDMS. The number of layers on the rod scales with the number of complete turns when rolling up the film (Fig. $4.8 \mathrm{c}$ ). We also recently reported this technique for fabricating metallo-dielectric multilayer rolls [38].

\section{“Origami” technique}

In this approach, the film is repeatedly folded onto itself. As with the cut-and-stack and roll-up methods, this technique proceeds from an initial polymer bilayer. However, here the thicknesses of the individual layers are one-half of the thicknesses required for DBR construction. Repeated folding of the entire stack onto itself leads to a multilayer stack with the number of individual layers increasing very rapidly with each folding operation. Sequential folding of the entire stack is achieved by a simple strategy: the slow immersion of a supported film partially releases the film onto the water surface up to a line where the film is physically fixed onto the substrate to prevent further release. By continuing to immerse the sample beyond this line, part of the floating film is redeposited onto the unreleased part of the double layer. Finally, slow retraction of the sample induces a second folding of the released film onto the folded part. In short, one folding cycle (displayed in the top of Fig. $4.8 \mathrm{~d}$ ) leads to a $z$-fold. The lower part of Fig. $4.8 \mathrm{~d}$ shows the initial bi-layer film, a multilayer after one folding cycle and a stack after two folding cycles. In this method, the final DBR is terminated by half-layers on both surfaces. If required, this is easily fixed by floating individual half-layers onto the final stack. The main advantage of the origami technique is the way the overall layer number in the stack $l_{f}$ scales with the number of folding operations $f$ as

$$
l_{f}=3^{f}-1
$$


These three techniques have the advantage of minimising the number of floating steps, reducing the likelihood of including defects (e.g. by dust particles) into the stack. The careful assembly of multilayers on the water surface or at the water meniscus prevents incorporation of air bubbles leading to defect-free areas of several square centimetres.

Although the presented samples are prototypes made by simple sequential films superposition, optimisation now in progress will allow larger area fabrication and potentially continuous roll-to-roll processes for the multilayer fabrication of elastomeric DBRs and cavities.

\subsubsection{Cavity design and modelling}

The optical performance of a fully elastomeric microcavity based on two stretchable DBRs surrounding a $\lambda / 2$ spacer was simulated using a standard transfer matrix technique. The results of these simulations were used to determine optimal film thicknesses that were targeted in DBR manufacture. Figure 4.9 shows a reflectivity spectrum of a DBR structure (black line) made from 30 bilayers with thicknesses $d_{1}=250 \mathrm{~nm}$, $d_{2}=150 \mathrm{~nm}$ and refractive indices $n_{1}=1.54, n_{2}=1.41$. The refractive indices of the superstrate and the substrate are 1.0 and 1.41, respectively. The red line is the calculated reflectivity of a microcavity consisting of two DBR stacks enclosing a cavity layer of thickness $d_{\mathrm{c}}=295 \mathrm{~nm}$ and refractive index $n_{\mathrm{c}}=1.54$. Figure $4.9 \mathrm{~b}$ shows calculated field distributions of the light in the cavity for the three different wavelengths marked in
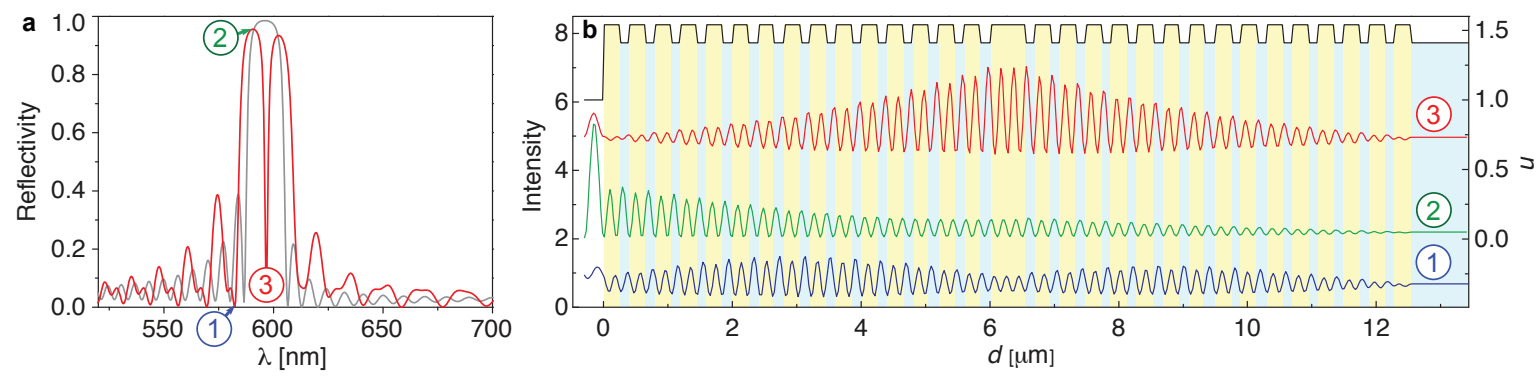

Figure 4.9: Simulated reflectivity spectra. a) Bragg mirror consisting of 15 double layers (black line) and a microcavity (red line) constructed from two 15 double layer DBRs surrounding a cavity spacer. b) Field distributions in the cavity structure at $582 \mathrm{~nm}(1), 590 \mathrm{~nm}(2)$ and $597 \mathrm{~nm}$ (3), marked in (a). 
Figure 4.9 a. Due to the small refractive index contrast, the cavity mode (3) penetrates extensively into the surrounding DBRs, on the order of $\bar{n} / \Delta n \simeq 11$ periods.

\subsubsection{Results and discussions}

Spectroscopic measurements were performed with a modified optical microscope to analyze the optical performance of the DBRs and the cavities at various locations on the films. A broad area of the sample is illuminated with an incandescent source, and a beamsplitter couples a fraction of the light reflected from the sample through a multimode fibre into an Ocean Optics USB4000 spectrometer. The setup is normalised and calibrated using reference mirrors, at a $10 \mu \mathrm{m}$ detection spot size. The samples were stretched in the optical setup using low profile, stepper motor controlled translation stages arranged to compensate any translation in position.

Upon stretching the multilayer sample, the reflectance peak reversibly shifts to lower wavelengths (Fig.4.10). The reflectance spectra in Fig. $4.10 \mathrm{~b}$ were acquired from a 20 layer DBR. The reflectivity peak at wavelength $\bar{\lambda}$, corresponds to predictions of the reflectivity spectrum based on the transfer matrix calculations. Assuming ideal rubber elasticity for both polymers and making use of Equation 4.2, the peak wavelength tuning is given by

$$
\bar{\lambda}(\varepsilon)=\frac{\bar{\lambda}_{0}}{\sqrt{1+\varepsilon}}
$$

where $\bar{\lambda}_{0}$ is the zero-strain peak wavelength and $\varepsilon$ the strain.

Repeated quasi-static stretching cycles did not alter the zero-strain peak wavelength of $681 \mathrm{~nm} \pm 2 \mathrm{~nm}$, nor its variation as a function of strain (Fig $4.10 \mathrm{c}$ ). The samples therefore exhibited full elastic recovery and the absence of ageing of the multilayer stacks for up to 12 cycles tested here. For the low frequency actuation of this study, the mechanical and optical response was instantaneous. Higher frequency actuation is conceptually limited only by molecular (Rouse) relaxation processes on sub-microsecond time scales [39], but dissipative losses potentially limit practical actuation speeds to 


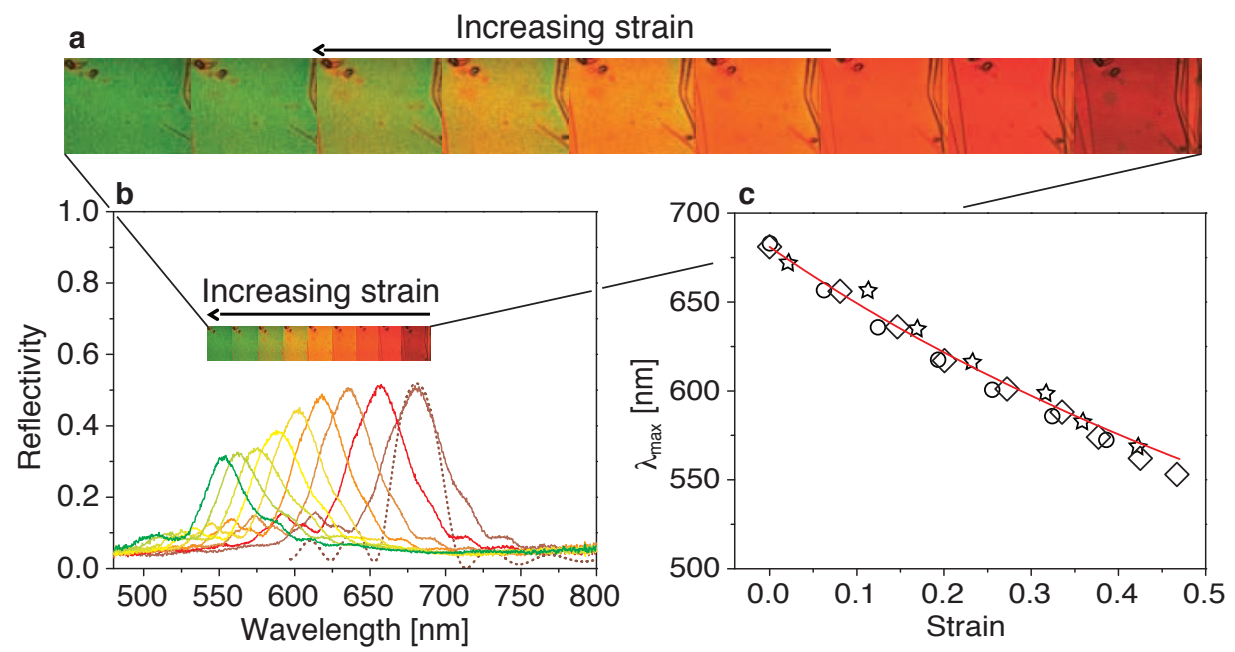

Figure 4.10: DBR tuning. a) As the multilayer stack is stretched, the colour changes rapidly and reversibly (image height: $140 \mu \mathrm{m}$ ). b) Reflectivity spectra of the multilayer stack at increasing strains. The dashed line shows the result of a reflectivity calculation for $\varepsilon=0$. c) Peak wavelength as a function of strain. Circles, diamonds and stars correspond to three consecutive strain cycles. The red line is the prediction of Equation (4.5).

less than $1 \mathrm{kHz}$. While the chemically cross-linked nature of PDMS prevents stress relaxation for static applied stresses, creep has been reported in PSPI if the applied stress exceeded two days at $30^{\circ} \mathrm{C}[40]$. This study would imply that the PSPI used here would creep if stressed for longer than one week at room temperature, but other elastomer systems can give even more stable performance.

With a refractive index difference of $\Delta n=0.13$, the maximum DBR reflectivity observed amounts to $\sim 50 \%$, which is only slightly less than the $52 \%$ predicted by model calculations. The peak width of $50 \mathrm{~nm}$ is larger than the $35 \mathrm{~nm}$ predicted by theory which is presumably due to imperfections and scattering in the multilayer caused by the current stack manufacture.

The decrease in intensity at higher strains probably arose from two effects : (1) The thinning of the stack at high strains may have moved the film out of the microscope focus, thereby drastically lowering the intensity of the incident light per unit area. (2) Small cracks appeared in this particular sample at strains above 35\%, which were not observed in multilayer cavities or multilayer sample with smaller stack numbers. They are a film-preparation artefact which was avoided in subsequent sample preparation. 

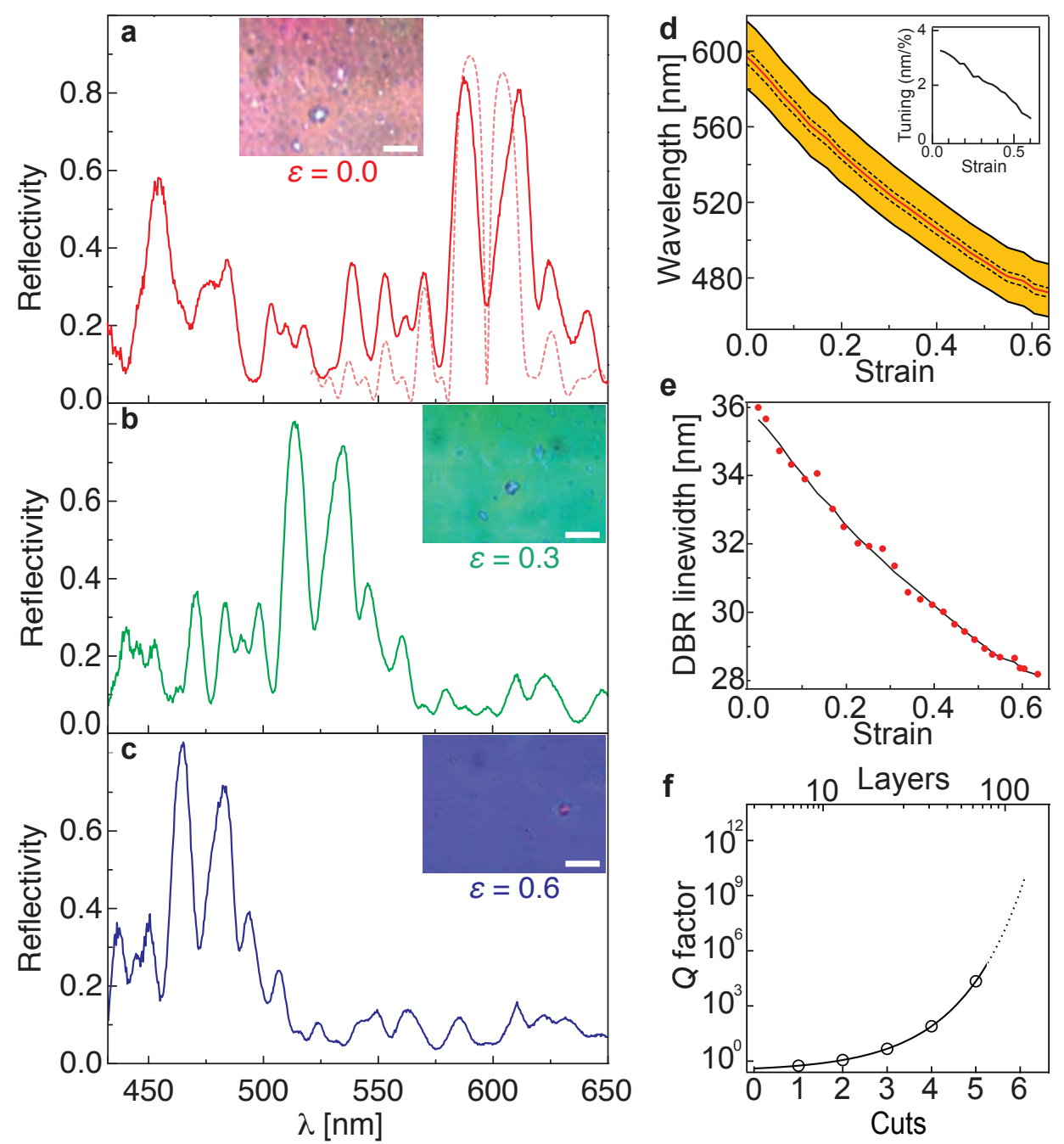

Figure 4.11: Elastomeric microcavity tuning as a function of strain. a) $\varepsilon=0$, b) $\varepsilon=0.3$ and (c) $\varepsilon=0.6$. Insets show micrographs of the film surface in reflection at each strain (scalebar: $20 \mu \mathrm{m}$ ). The dashed line in (a) is a calculation for $\varepsilon=0$. d) DBR mirror stop band (black lines) and microcavity mode (red line) vs applied strain. The fits for both are identical and follow Equation 4.5. The inset shows the rate of wavelength change with applied strain. e) DBR bandwidth and fit (line) vs applied strain. f) Theroetically predicted $Q$-factor of the microcavities as a function of the number of cut-and-stack operations.

The optical performance of a fully elastomeric microcavity based on two stretchable DBRs surrounding a $\lambda / 2$ PSPI spacer layer is shown in Figure 4.11. A clear cavity mode is present in the middle of the DBR stopband, with the peak reflectivity now increasing to $R_{\max } \approx 85 \%$ (compared to a predicted reflectivity of $89 \%$ ). The change in shape of the cavity dip is minimal upon stretching, with both DBR mirrors and the cavity mode following identical tuning (Fig.4.11d). Significantly, the wavelength 
of the cavity resonance follows Poisson's law (Equation 4.5) very accurately for strains exceeding $\varepsilon=0.65$, which enables precise tuning of the cavity mode. The absolute reflectivities also do not decrease with stretching, which indicates that the thickness ratio of the PSPI and PDMS layers remains constant. Hence, although the mirror bandwidth of these DBRs is five times smaller than their tuning range, the cavity mode remains spectrally aligned with the stopband at all strains (Fig. 4.11d). This bandwidth is as expected from theory, $\Delta \lambda=\frac{2}{\pi} \bar{\lambda} \frac{\Delta n}{\bar{n}} \simeq 35 \mathrm{~nm}$ at low strain, and decreasing as predicted on stretching (Fig. 4.11e) [41]. Wider free spectral ranges can be achieved by increasing the refractive index contrast in the multilayer, for example by doping one of the rubbers with nanoparticles [42].

The quality factor of the cavity $Q \simeq 55$ is independent of the applied strain. Provided the defect density can be limited by minimising the number of floating steps using the "origami", roll-up or "cut and stack" techniques, we emphasise an extremely favourable exponential scaling of the cavity $Q$ with the number of "cut and stack" operations $c$ used in cavity construction, which rapidly increases the number of layers $N=2^{c+1}$. For small $\Delta n$, the resulting $Q$-factor increases as

$$
Q=Q_{0} \exp \left\{2 N \frac{\Delta n}{\bar{n}}\right\}
$$

where $Q_{0}=\frac{m \pi}{4}\left(\frac{n_{0}}{n_{\mathrm{s}}}\right), n_{0}, n_{\mathrm{s}}$ are the superstrate and substrate refractive indices, and $m$ is the number of half-wavelengths that fit inside the microcavity (Fig. $4.11 \mathrm{f}$ ). While the $Q$ factor of the cavities is not yet very high, the extrapolation of Equation 4.6 illustrates the potential of our technique. Cavities with higher $Q$-factors can be built by improving multilayer manufacture in applying the "cut and stack", "roll up" or "origami" techniques to drastically decrease the number of floating steps, while minimising the inclusion of imperfections. In principle the limit on the optical quality of the cavities is the roughness of the initial bilayer, which can be less than $1 \mathrm{~nm}$, when optimised.

Future study will have to address the maximum number of cycles at high and low frequencies and static stress relaxation. Based on the large body of literature about the two commodity rubbers used in this work, we expect excellent repeatability and 
reproducibility for a large number of cycles and frequency range. Our limited testing did not reveal any signs of sample delamination or other signs of physical degradation of the rubber multilayers.

A straightforward extension of our elastomeric microcavities is the inclusion of a dye within the cavity to manufacture tuneable emitters, in which the output colour is controlled by the local strain, which can be applied by a standard micro-actuator. These optically-pumped polymer cavity LEDs indeed give narrowband enhanced and beamed light emission.

A more intriguing possibility is the creation of stable laterally localised microcavities in which the light is confined in all spatial directions. Normally the production of nonplanar DBRs is problematic, particularly on the micrometre length scale [43]. Here we demonstrate a simple cavity confinement by compressing an elastomeric flexible DBR on a stamped PDMS grating (4 $\mu \mathrm{m}$ pitch, $250 \mathrm{~nm}$ high square grooves) to create curved DBRs on the wavelength scale (Fig. 4.12). Subsequent AFM imaging shows that the DBR has a height variation of $30 \mathrm{~nm}$ (Fig. $4.12 \mathrm{~b}$ ), which is observed in the transmission

a

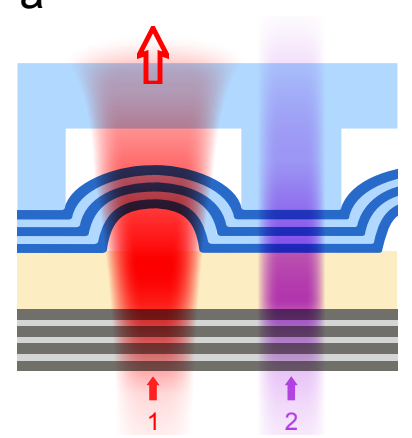

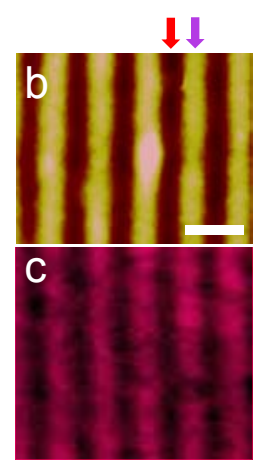

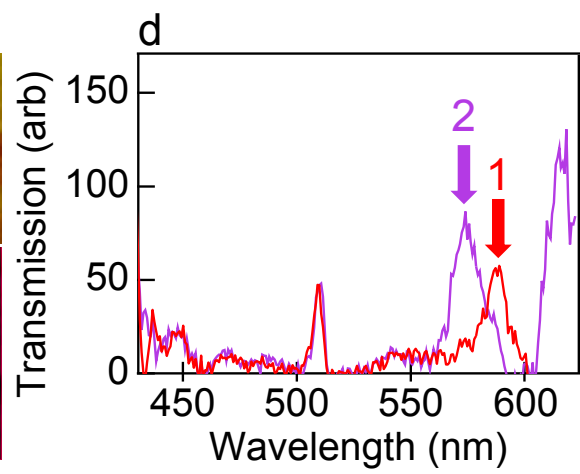

Figure 4.12: Microcavity formed from a flat lower DBR on glass, and an upper elastomeric DBR on a PDMS grating. a) Schematic cross-section: a DBR multilayer is placed on a PDMS line grating (pitch: $4 \mu \mathrm{m}, 250 \mathrm{~nm}$ high square grooves) and the assembly is put into contact with a commercial planar DBR covered by a $206 \mathrm{~nm}$ thick PDMS spacer layer. Stable cavity modes form at position 1. b) AFM image of the elastomeric DBR on the PDMS line grating (height scale: $30 \mathrm{~nm}$, scalebar: $5 \mu \mathrm{m}$ ), and c) transmission image of the full microcavity in infra-red light. d) Selected transmission spectra from line scans across the sample, showing the different confined optical modes at points indicated by red and purple arrows in (a),(b). The peak at $510 \mathrm{~nm}$ originates from a defect in the lower DBR only. 
intensity through the microcavity (Fig. 4.12c). The cavity defect layer is constructed by spin coating a Teflon ${ }^{\circledR} \mathrm{AF}$ spacer layer of thickness $\simeq \lambda / 2 n=206 \mathrm{~nm}$ on the lower multilayer dielectric DBR. Assembling the full cavity is extremely simple since dust and imperfections, that normally inhibit effective microcavity assembly for small spacings, here only locally push apart the upper and lower DBRs. Away from such local defects the same optical characteristics can be observed all across this sample. Using confocal microscopy we observe the resulting cavity mode, whose resonant wavelength clearly tunes periodically across the grating pattern. In the unstable cavity configuration (the shorter cavity 2 in Fig. 4.12 a) the cavity mode blue-shifts as expected, and doubles in linewidth.

We envisage creating sheets of such microcavities by stretching the elastomeric DBR over an array of separately addressable holes to allow for control of individual cavity wavelengths. This enables applications in biosensing, microfluidics, and photonics.

In summary we demonstrate the fabrication of rubber-based, stretchable Bragg mirrors and Bragg microcavity filters by simple, scaleable processing techniques. Upon stretching the devices, the reduction in layer thickness blue-shifts the DBR reflection stopband and the microcavity resonant mode. Shifts in wavelength across the entire visible spectrum are achieved by stretching the devices by more than $60 \%$. Stretch-tuneable DBRs can thus be directly employed for optical stress-strain sensing applications, in LED and lasing applications with broadband emitters to allow for wide range wavelength tuning, and in a host of novel applications.

\subsection{Conclusion}

Planar one-dimensional photonic structures, similar to the multilayer found in the cuticle of the Japanese Jewel beetle have been manufactured using different routes in the context of this thesis. Although some production issues remain to be solved, PS-Teflon ${ }^{\circledR} \mathrm{AF}$ multilayer structures show a promising optical behaviour for use as selective reflectors or bandpass filters and Bragg mirrors for fully organic resonance 
cavity enhanced LEDs. Tuning of the optical properties of elastomeric Bragg reflectors and resonance cavities has been demonstrated, and future work is directed towards the production of dye-loaded elastomeric resonance cavities and the study of controlled three-dimensional deformation of planar elastic structures on the micron scale to create taylor made, tuneable multidimensional resonance cavities or piezochromic devices. 



\section{References}

[1] Joannopoulos, J. D., Johnson, S. G., Winn, J. N. \& Meade, R. D. Photonic crystals: Molding the flow of light (Princeton University Press, 2008).

[2] Riihelä, D., Ritala, M., Matero, R. \& Leskelä, M. Introducing atomic layer epitaxy for the deposition of optical thin films. Thin Solid Films 289, 250-255 (1996).

[3] Weber, M., Stover, C., Gilbert, L., Nevitt, T. \& Ouderkirk, A. Giant birefringent optics in multilayer polymer mirrors. Science 287, 2451-2456 (2000).

[4] Mönch, W., Dehnert, J., Jaufmann, E. \& Zappe, H. Flory-huggins swelling of polymer Bragg mirrors. Appl. Phys. Lett. 89, 164104 (2006).

[5] Meyerhofer, D. Characteristics of resist films produced by spinning. J. Appl. Phys. 49, 3993-3997 (1978).

[6] Ho, P., Thomas, D., Friend, R. \& Tessler, N. All-polymer optoelectronic devices. Science 285, 233 (1999).

[7] Schubert, E., Wang, Y., Cho, A., Tu, L. \& Zydzik, G. Resonant cavity light emitting diode. Appl. Phys. Lett. 60, 921-923 (1992).

[8] Sommer, M. Novel Semiconductor Block Copolymers for Organic Electronic Devices: Synthesis, Properties and Applications. Ph.D. thesis, University of Bayreuth (2009).

[9] Hüttner, S. Donor-Acceptor Block Copolymers in Organic Electronics - Spectroscopy, Charge Transport, Morphology and Device Application. Ph.D. thesis, University of Bayreuth (2010).

[10] Song, M., Kabra, D., Wenger, B., Friend, R. \& Snaith, H. Optically-pumped 
lasing in hybrid organic-inorganic light-emitting diodes. Adv. Funct. Mater. 19, 2130-2136 (2009).

[11] Morii, K., Kawase, T. \& Inoue, S. High efficiency and stability in air of the encapsulation-free hybrid organic-inorganic light-emitting diode. Appl. Phys. Lett. 92, 213304 (2008).

[12] Lacour, S., Wagner, S., Huang, Z. \& Suo, Z. Stretchable gold conductors on elastomeric substrates. Appl. Phys. Lett. 82, 2404 (2003).

[13] Li, T., Huang, Z., Suo, Z., Lacour, S. \& Wagner, S. Stretchability of thin metal films on elastomer substrates. Appl. Phys. Lett. 85, 3435 (2004).

[14] Janata, J. \& Josowicz, M. Conducting polymers in electronic chemical sensors. Nature Mater. 2, 19-24 (2003).

[15] Gustafsson, G. et al. Flexible light-emitting diodes made from soluble conducting polymers. Nature 357 (1992).

[16] Malinauskas, A. Chemical deposition of conducting polymers. Polymer 42, 3957$3972(2001)$.

[17] Born, M. \& Wolf, E. Principles of Optics (Cambridge University Press, 2005).

[18] Abeles, F. Recherches sur la propagation des ondes électromagnétiques sinusoïdales dans les milieux stratifiés. Application aux couches minces. Ann. Phys. (series 12) 5, 596-640 (part I), 706-784 (part II) (1950).

[19] Heavens, O. Optical properties of thin solid films (Dover Publications, 1965).

[20] Fink, Y. et al. A dielectric omnidirectional reflector. Science 282, 1679-1682 (1998).

[21] Huffaker, D. \& Deppe, D. Low threshold vertical-cavity surface-emitting lasers based on high contrast distributed Bragg reflectors. Appl. Phys. Lett. 70, 17811783 (1997). 
[22] Jiang, H. et al. Plasma polymerized multi-layered photonic films. Chem. Mater. 15, 340-347 (2003).

[23] Edrington, A. et al. Polymer-based photonic crystals. Adv. Mater. 13, 421-425 (2001).

[24] Komikado, T., Inoue, A., Masuda, K., Ando, T. \& Umegaki, S. Multi-layered mirrors fabricated by spin-coating organic polymers. Thin Solid Films 515, 38873892 (2007).

[25] Martinu, L. \& Poitras, D. Plasma deposition of optical films and coatings: A review. J. Vac. Sci. Tech. A 18, 2619-2645 (2000).

[26] Setzu, S., Ferrand, P. \& Romestain, R. Optical properties of multilayered porous silicon. Mater. Sci. Eng. B 69, 34-42 (2000).

[27] Gellermann, W., Kohmoto, M., Sutherland, B. \& Taylor, P. Localization of light wave in Fibonacci dielectric multilayers. Phys. Rev. Lett. 72, 633-636 (1994).

[28] Stanley, R., Houdre, R., Oesterle, U., Gailhanou, M. \& Ilegems, M. Ultrahigh finesse microcavity with distributed Bragg reflectors. Appl. Phys. Lett. 65, 18831885 (1994).

[29] Langer, R. et al. High-reflectivity GaN/GaAlN Bragg mirrors at blue/green wavelengths grown by molecular beam epitaxy. Appl. Phys. Lett. 74, 3610-3612 (1999).

[30] Domash, L., Wu, M., Nemchuk, N. \& Ma, E. Tunable and switchable multiplecavity thin film filters. J. Lightwave Technol. 22, 126-135 (2004).

[31] Mönch, W., Dehnert, J., Prucker, O., Rühe, J. \& Zappe, H. Tunable Bragg filters based on polymer swelling. Appl. Opt. 45, 4284-4290 (2006).

[32] Kang, Y., Walish, J., Gorishnyy, T. \& Thomas, E. Broad-wavelength-range chemically tunable block-copolymer photonic gels. Nature Mater. 6, 957-960 (2007).

[33] Sandrock, M. et al. A widely tunable refractive index in a nanolayered photonic material. Appl. Phys. Lett. 84, 3621-3623 (2004). 
[34] Kimura, M., Okahara, K. \& Miyamoto, T. Tunable multilayer-film distributedBragg-reflector filter. J. Appl. Phys. 50, 1222-1225 (1979).

[35] Li, J. et al. Reversibly strain-tunable elastomeric photonic crystals. Chem. Phys. Lett. 390, 285-289 (2004).

[36] Shojaei-Zadeh, S., Swanson, S. \& Anna, S. Highly uniform micro-cavity arrays in flexible elastomer film. Soft Matter 5, 743-746 (2009).

[37] Pursiainen, O. et al. Nanoparticle-tuned structural color from polymer opals. Opt. Express 15, 9553-9561 (2007).

[38] Gibbons, N., Baumberg, J., Bower, C., Kolle, M. \& Steiner, U. Scalable cylindrical metallo-dielectric metamaterials. Adv. Mater. 21, 3933-3936 (2009).

[39] Rubinstein, M. \& Colby, R. H. Polymer Physics (Oxford University Press, 2003).

[40] Hotta, A., Clarke, S. M. \& Terentjev, E. M. Stress relaxation in transient networks of symmetric triblock styrene-isoprene-styrene copolymer. Macromolecules $\mathbf{3 5}$, 271-277 (2002).

[41] Kavokin, A. \& Baumberg, J. Microcavities (Oxford University Press, 2007).

[42] Caseri, W. Nanocomposites of polymers and metals or semiconductors: Historical background and optical properties. Macromol. Rapid Commun. 21, 705 - 722 (2000).

[43] Pennington, R., D’Alessandro, G., Baumberg, J. \& Kaczmarek, M. Spectral properties and modes of surface microcavities. Phys. Rev. A 79, 043822 (2009). 


\section{Chapter 5}

\section{Microfabrication of photonic structures with higher dimensionality}

The manufacture of periodic micro- and nanostructures on macroscopic areas is a challenging task. Serial nano-production techniques such as electron or ion beam lithography [1-4] are well suited to make structures with feature sizes below $10 \mathrm{~nm}$, but the patterned areas are usually only several $\mu^{2}$ in size. Direct laser writing, where a laser is used to write patterns into a polymer resist, can be used to produce two- or threedimensional structures with a resolution down to $\sim 100 \mathrm{~nm}[5,6]$. Like electron or ion beam lithography, this method is inherently serial and the time $t$ needed for producing a pattern scales as $t \sim l^{\alpha}$ with $l$ being the side length of the pattern and $\alpha$ the number of relevant dimensions in the fabricated photonic structure.

Two-dimensional patterns with sub-100 nm resolution in polymer resists can nowadays be fabricated by photolithography, which is the standard nano-patterning technique in semiconductor industry [7]. With this method high throughput can be achieved, but sophisticated tricks [8-10] are needed to achieve this resolution and usually highly specialised, expensive equipment for immersion photolithography, deep or extreme ultraviolet photolithography $[11,12]$ is necessary. Conventional optical lithography is restricted to the production of two-dimensional structures and several thin film deposition, lithographic and registering steps have to be performed in order to create more complex concave or convex patterns. 
In this thesis, two different, highly parallel techniques have been investigated for the production of periodic patterns with micro - to nanometre resolution:

- Template-assisted ion milling for the patterning of polymers, metal-oxides, glass and silicon.

- Nano-imprinting for the transfer of patterns into soft materials.

Both techniques are described in Chapter 3. Here, the resulting micro- and nanostructures are presented.

\subsection{Template-assisted ion milling}

\subsubsection{The ion milling template}

Colloidal monolayers with mono-crystalline domains of several $100 \mu^{2}$ in size were deposited on various substrate materials using the colloid blading technique [13] (Chapter 3.3.1). A macroscopic coverage of several $\mathrm{cm}^{2}$ can easily be achieved. The used polystyrene microspheres, purchased from Duke Scientific Standards ${ }^{\mathrm{TM}}$ have diameters of $1 \mu \mathrm{m}, 2 \mu \mathrm{m}, 3 \mu \mathrm{m}$ or $5 \mu \mathrm{m}$ with a relative standard deviation of about $1 \%$. Smaller
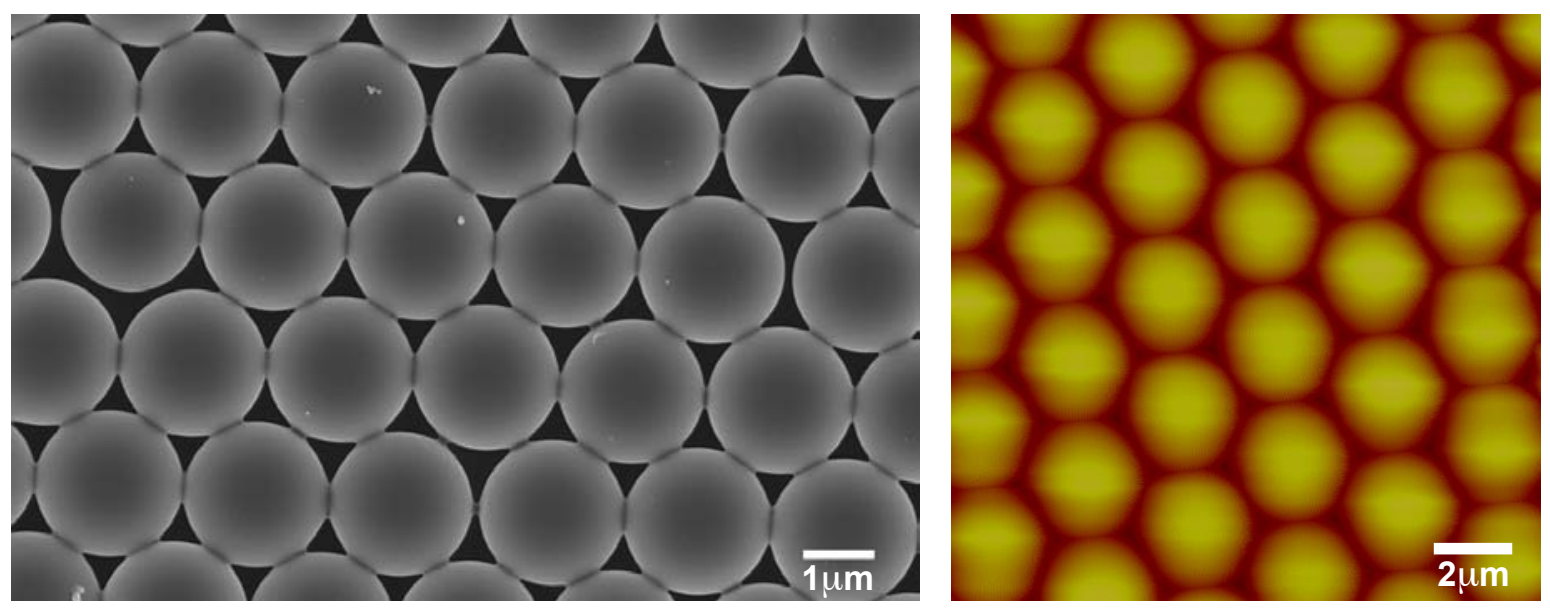

Figure 5.1: Colloidal templates. a) Scanning electron microscopy image of a monolayer of hexagonally packed, $2 \mu \mathrm{m}$-sized colloids. b) Atomic force microscopy image of a colloidal layer with a micro-sphere diameter of $2 \mu \mathrm{m}$. 
colloids with diameters down to $400 \mathrm{~nm}$ have been deposited by spin coating, but the resulting polycrystalline monolayers have small domain sizes. Standard substrates were silicon, gold- or chrome-coated silicon, polymer layers, or glass.

\subsubsection{Micro-cones}

After the substrate has been masked with the colloid template, it is patterned by argon ion milling. Shape and height of the produced features depend on the milling parameters, namely the ion beam current, beam voltage and milling time, whereas the periodicity of the patterns is determined by the mask.

Ion beam current and voltage, which determine the number of ions hitting the sample in a second and their speed/energy upon impact, are usually kept constant during the milling process and the processing time is the only adjustable variable ${ }^{1}$. More information on the technique can be found in Chapter 3 .

To avoid excessive heating, deformation and melting of the mask colloids during exposure to the ion beam (Fig. 5.2), a milling run is split into several intervals of $5 \mathrm{~min}$ milling followed by a 2 min cooling step. For substrates with low thermal conductivity, such as polymers or glass, the intervals are shortened to $2 \mathrm{~min}$ and $2 \mathrm{~min}$.

\footnotetext{
${ }^{1}$ The milling current can fluctuate by several $\mathrm{mA}$ in the used device during the milling process, which has an influence on the milling rate.
}

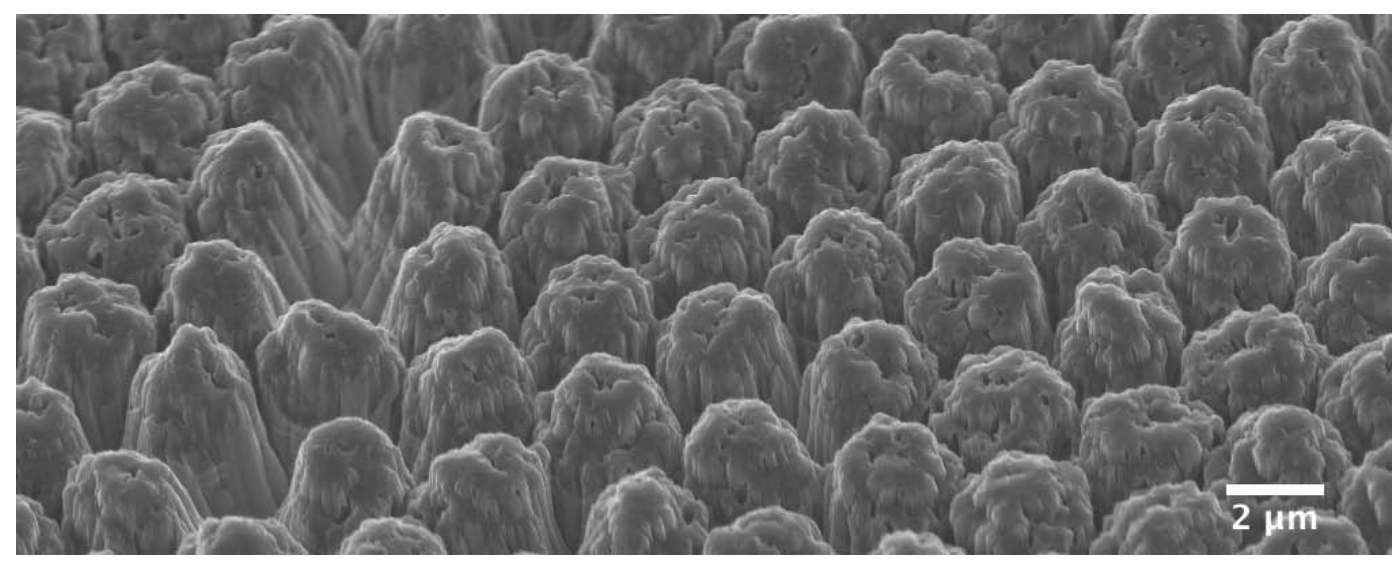

Figure 5.2: A colloid template that has been melted during ion milling. 

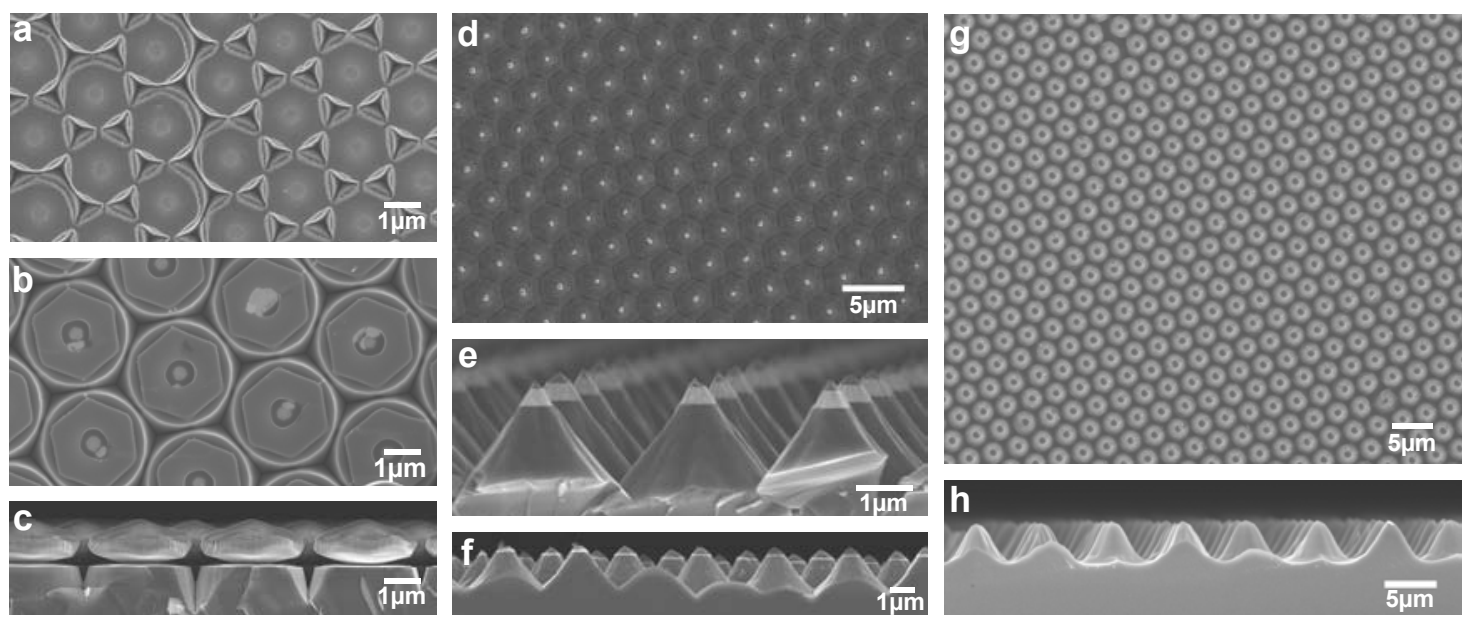

Figure 5.3: Micro-stubs and -cones. a) Silicon stubs with a diameter of $2 \mu \mathrm{m}$ topped by interconnected $50 \mathrm{~nm}$ thick gold hexagons. b) Unconnected gold hexagons on top of $3 \mu \mathrm{m}$-sized stubs. c) The $3 \mu \mathrm{m}$-wide stubs in cross-section with the colloid template still sitting on top. d) Array of hexagonally arranged $1.5 \mu \mathrm{m}$ high silicon microcones with a $230 \mathrm{~nm}$ gold topping and a diameter of $3 \mu \mathrm{m}$ at base level. e) The cones from (d) in cross-section. The remainders of the colloidal template can be seen as tips on the gold toppings. f) Similar silicon micro-cones with $50 \mathrm{~nm}$-thick, $0.8 \mu \mathrm{m}$-wide gold discs on top, covered by the remaining polymer caps. g) Hexagonal array of $1.5 \mu \mathrm{m}$ high glass cones with a peak to peak distance of $3 \mu \mathrm{m}$. h) Cross-section of the glass cones from (g).

Following different protocols, various hexagonally arranged cone and stub structures have been produced (Fig 5.3). The aspect ratio (the height-to-width ratio) of sharptipped cones is $\sim \frac{1}{2}$ (Fig. 5.4 a), but can be smaller, if the milling is continued beyond the completed removal of the colloidal mask (Fig 5.5). Complex patterns composed of hexagonally arranged protrusions with lattice spacings corresponding to the diameter $d$ of the templating colloids and small holes with lattice spacings of $\frac{d}{\sqrt{3}}$ have been fabricated. The hexagonal patterns of protrusions and holes are rotated by $30^{\circ}$ with respect to each other (see schematic in Fig. $5.4 \mathrm{~b}$ and Fig. $5.5 \mathrm{a}$ where blue and green hexagons mark the unit cells of tips and holes).

Since polymer colloids or other photolithographically patterned polymer templates are easily applied to and also removed from various substrates, they represent reasonably good masking materials for ion beam patterning procedures. However, the milling rates of most polymers, silicon, glass and other metal oxides are comparable (Table 5.1), 
a

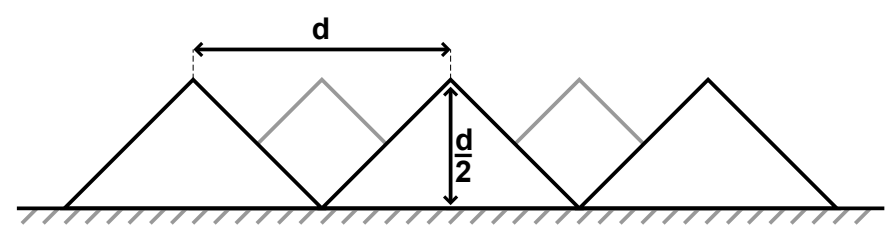

b

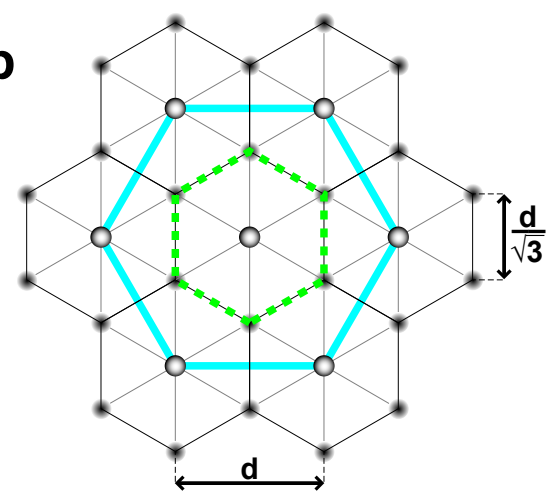

Figure 5.4: Schematic of the cone arrangement. a) Side view of cones made by using colloids with a diameter of $d$ leading to the shown aspect ratio. b) Top view, showing the hexagonal pattern of cone tips (light blue) and small holes between the cones (green, dashed).
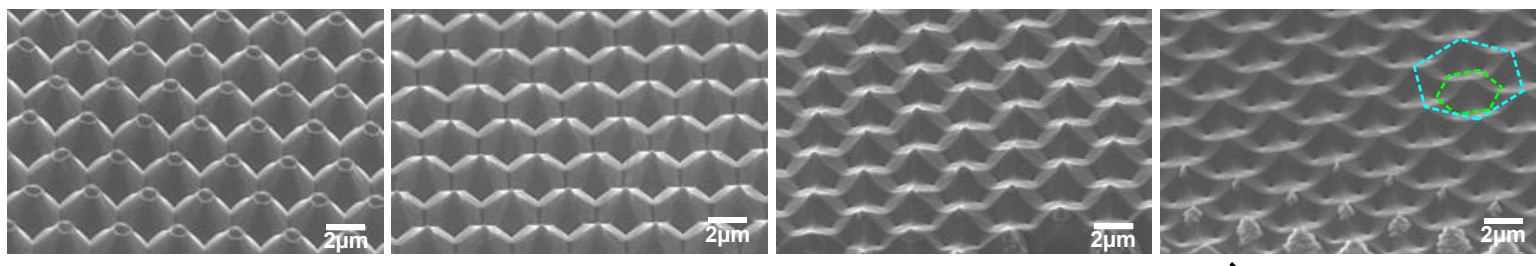

Increasing milling time

Figure 5.5: Micro-cones with different tip-geomtries. a) Micro-cones with flat gold tops and polymer caps, aspect ratio $\sim \frac{1}{2}$. b) Micro-cones with sharp tips, aspect ratio $\sim \frac{1}{2}$. c) Micro-cones with sharp tips and aspect ratio $<\frac{1}{2}$. c) Micro-cones with sharp tips, aspect ratio $<\frac{1}{4}$. The dashed blue and green hexagons show the two different hexagonal sub-structures of peaks and holes in the pattern.

which complicates the creation of patterns with very high aspect ratios ${ }^{2}$. Noble metals (gold, silver, ...) show milling rates that are one order of magnitude higher, allowing the realisation of high aspect ratio structures by polymer template-assisted argon ion milling in these materials. Milling times are around 60-70 min to produce cone structures using $3 \mu \mathrm{m}$-sized colloids as a template. Figure 5.6 displays a silicon substrate covered with a colloidal monolayer, imaged several times during one milling process.

\footnotetext{
${ }^{2}$ Preliminary experiments with cross-linked SU8 photoresist as a patterning material suggest that this particular resist has a milling rate which is an order of magnitude lower compared to the polymer colloids, but this has to be investigated in more detail.
} 


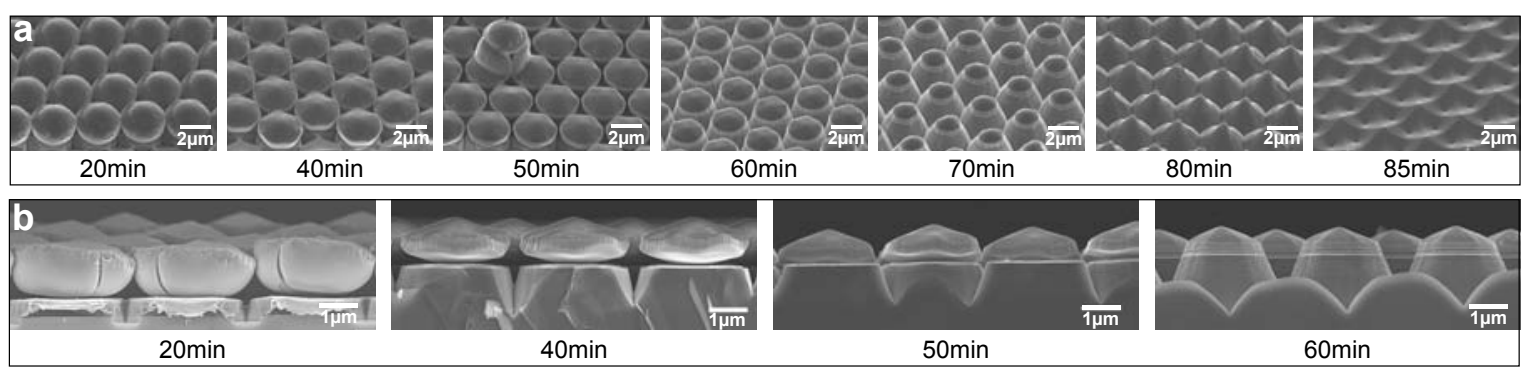

Figure 5.6: Development of the cone patterns as a function of milling time. a) Surface of the colloid-masked silicon substrate for increasing milling times. b) Cross-section of the same sample taken at different times during the milling process.

\begin{tabular}{lcc}
\hline Material & Milling rate $(\mathbf{n m} / \mathbf{m i n})$ & source \\
\hline $\mathrm{Si}$ & 38 & {$[14]$} \\
$\mathrm{SiO}_{2}$ & 39 & {$[14]$} \\
& 37 & {$[15]$} \\
$\mathrm{Al}_{2} \mathrm{O}_{3}$ & 10 & {$[14]$} \\
$\mathrm{Au}$ & 170 & {$[14]$} \\
& 108 & {$[15]$} \\
$\mathrm{Ag}$ & 220 & {$[14]$} \\
& 180 & {$[15]$} \\
$\mathrm{Pt}$ & 88 & {$[14]$} \\
$\mathrm{Cr}$ & 58 & {$[14]$} \\
& 53 & {$[15]$} \\
$\mathrm{PS}$ colloids & $\sim 20$ & experimental result \\
\hline
\end{tabular}

Table 5.1: Milling rates of different materials. Rates correspond to argon milling at $500 \mathrm{~V}$ beam voltage and $\sim 1 \mathrm{~mA} / \mathrm{cm}^{2}$ beam current.

\subsubsection{Gold crowns on micro-cones}

The deposition of a functional material (such as gold) on the silicon substrate prior to the deposition of the colloid mask allows the creation of small, flat islands on top of the cones (Fig. 5.3). These islands maintain a hexagonal shape, as long as the slowly degrading colloids have not completely melted down onto the substrate surface, which happens after $\sim 40-50 \mathrm{~min}$ of the ion milling process (Fig. 5.6).

A different kind of metallic structure can be manufactured on top of the silicon cones by electro-deposition of metals in the interstices of the colloid monolayer [16-19] prior to the ion milling. Gold "micro-crowns" of various shapes and "cup-sizes" have been pro- 

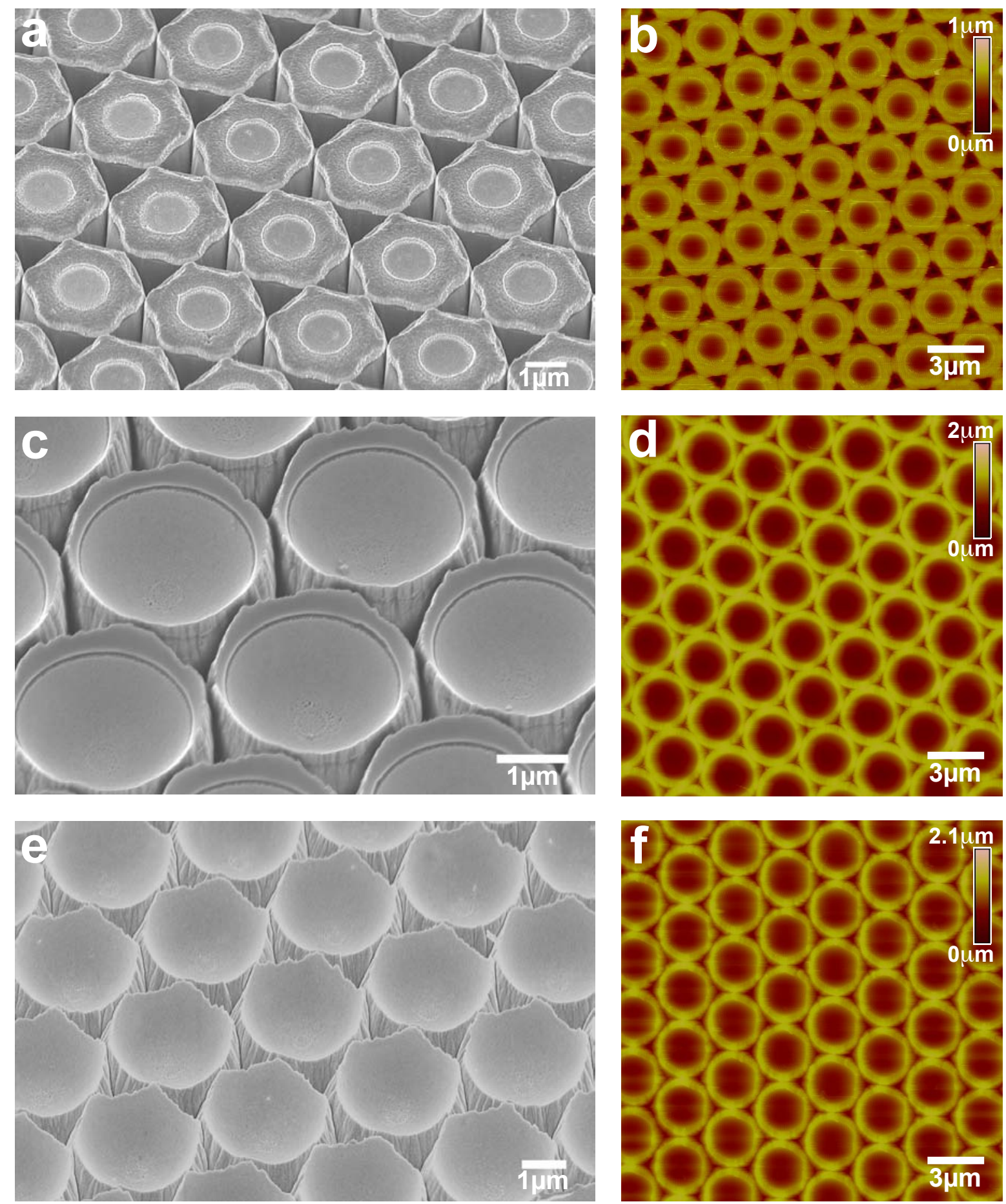

Figure 5.7: Gold micro-crown structures on silicon micro-stubs. a) Shallow gold crowns. b) AFM image of the structure in (a). c) Gold dishes with a rim. d) AFM image of the structure in (c). e) Deep gold bowls. f) AFM image of the structure in (e).

duced on top of silicon stubs ranging in diameter from $3 \mu \mathrm{m}$ down to $600 \mathrm{~nm}$. Electrodeposition of gold in the interstitial regions of the colloidal template was done by 


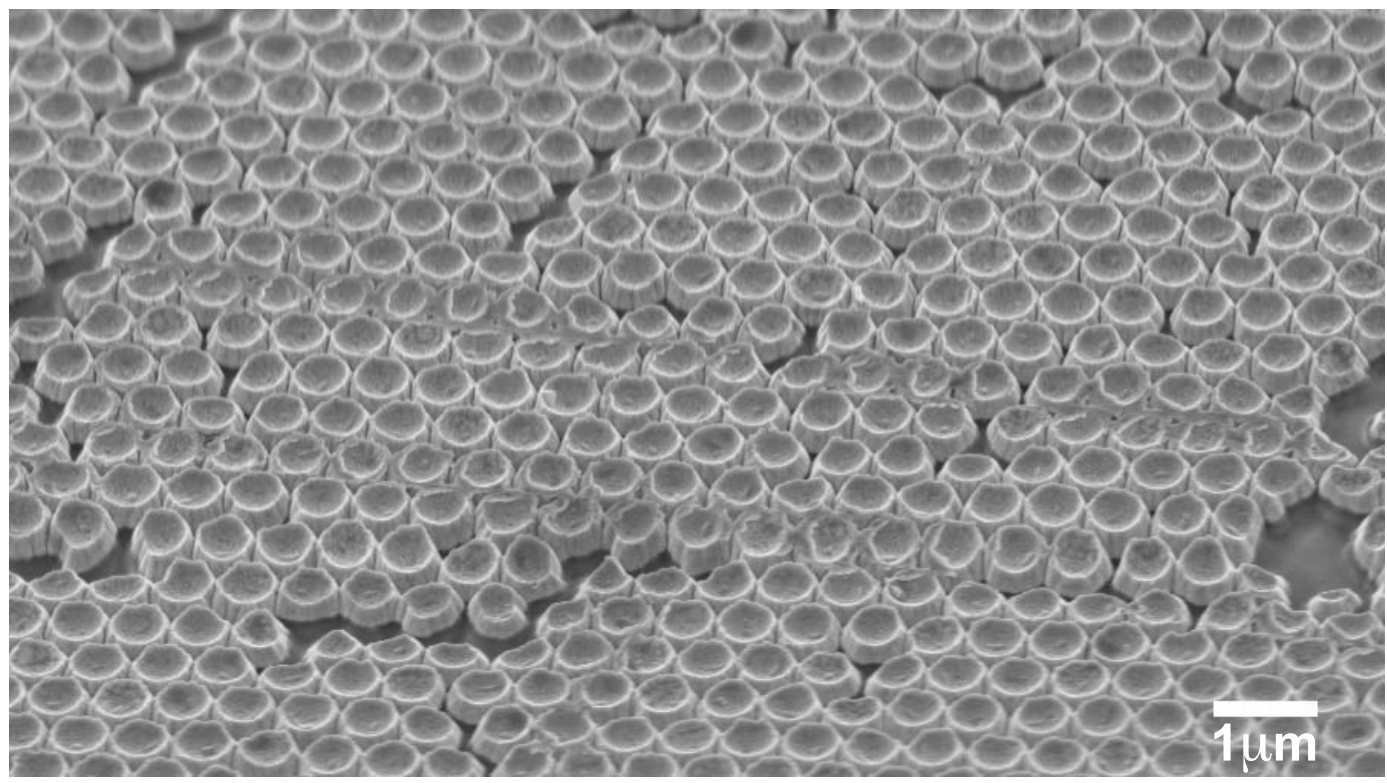

Figure 5.8: Gold nano-crown structures on silicon. A first prototype of gold dishes with $\sim 600 \mathrm{~nm}$ diameter. Creation of long range ordered arrays of gold crowns with uniform shape and size becomes more challenging at this length scale.

Dr. S. Mahajan from the NanoPhotonics group at the Cavendish Laboratory, who subsequently used the crown-structures as micro-reservoirs for the detection of organic molecules by surface enhanced Raman scattering (SERS [20]). In this context, gold structures varying from a dish-like shape with wide rims to a bowl-like profile with no rim have been manufactured (Fig. 5.7). Mild Raman enhancements due to plasmonic effects on the patterned gold surface have been shown for structures of $2 \mu \mathrm{m}$ periodicity. To realise the field enhancement [21, 22] that is needed for single molecule detection by surface enhanced Raman scattering, the gold structures have to be manufactured on a smaller length scale. A first prototype of gold dishes with $600 \mathrm{~nm}$ diameter is displayed in Figure 5.8.

\subsubsection{Multilayer patterning by ion milling}

The ion milling technique can be used to pattern polymer multilayer stacks that are masked by colloid monolayers. A thin gold film is deposited by evaporation on a multilayer of polystyrene (PS) and Teflon ${ }^{\circledR}$ AF supported by a silicon or glass sub- 
strate. Subsequently, a colloidal monolayer is assembled on the gold film and the sample is processed in the ion miller for $\sim 20 \mathrm{~min}$. Finally, the gold film is dissolved in a mild bromine solution [23] accompanied the colloid lift-off, which is assisted by careful ultra-sonication. This way, patterned stacks of five alternating polymer layers have been fabricated on silicon (Fig. 5.9).

The milling rates of the polymer colloidal mask and the polymer multilayer materials are comparable, making it difficult to create patterned multilayers with a high number of individual layers.
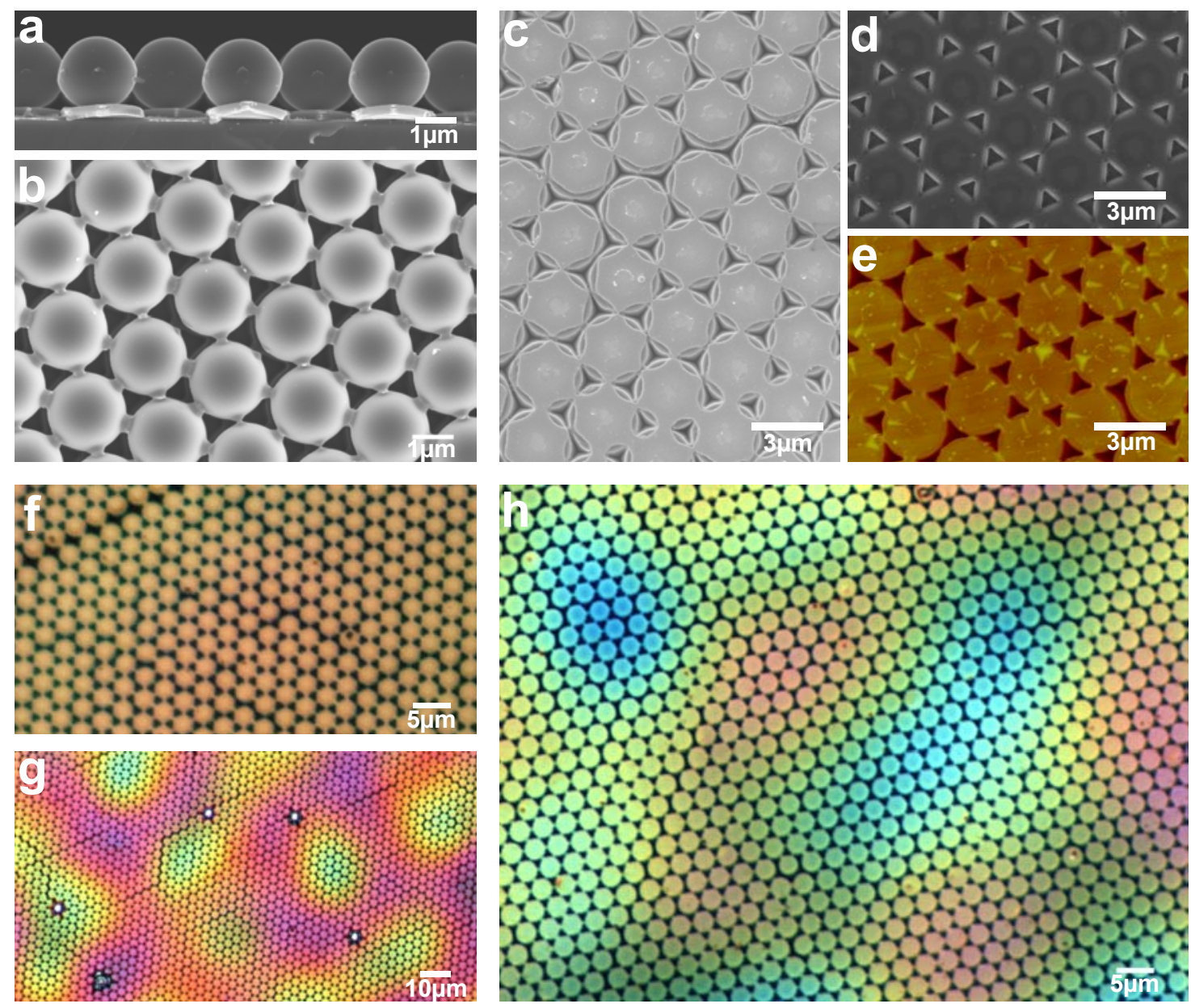

Figure 5.9: Multilayer patterning by ion milling. a), b) Cross-section and topview of a colloid-masked, gold-coated PS-Teflon ${ }^{\circledR}$ AF multilayer on silicon after ion milling. c) The sample after removal of the colloid mask and d) after removal of the gold film. e) The patterned polymer film imaged with an AFM. f) Microscopic image of the patterned polymer multilayer coated with the gold film. g) h) Microscopic images of two patterned multilayers after the removal of colloids and gold film for two different magnifications. 
Metal oxides such as zinc oxide $(\mathrm{ZnO})$, titania $\left(\mathrm{TiO}_{2}\right)$, zirconia $\left(\mathrm{ZrO}_{2}\right)$, alumina $\left(\mathrm{Al}_{2} \mathrm{O}_{3}\right)$, and silica $\left(\mathrm{SiO}_{2}\right)$ are transparent as thin films and cover a broad refractive index range. These materials are widely employed in the production of optical components [24-27]. The difference in milling rates of the colloidal monolayer masks and metal oxides is not significantly larger compared to the organic multilayer materials. However, due to the high refractive index contrast that can be achieved in metal oxide multilayers, significantly less individual layers are needed to assure a high reflectivity. This means, that metal oxide multilayer stack are thinner than comparable organic optical elements and they do not require high aspect ratio patterning. Consequently, metal oxides are very suitable for the production of patterned multilayer optical devices by polymer template-assisted ion milling.

A prototype of an inorganic patterned multilayer resonance cavity structure on glass has been produced by template-assisted ion milling. The cavity structure was fabricated by atomic layer deposition of titania $\left(\mathrm{TiO}_{2}\right)$ and alumina $\left(\mathrm{Al}_{2} \mathrm{O}_{3}\right)$ at $200^{\circ} \mathrm{C}$. The sample appears violet in reflection and green in transmission (Fig. 5.10 a). The thickness of the titania and alumina films in the distributed Bragg reflectors (DBRs) was determined from scanning electron micrographs and found to be $d_{\mathrm{TiO}_{2}}=(31 \pm 5) \mathrm{nm}$ and $d_{\mathrm{Al}_{2} \mathrm{O}_{3}}=(83 \pm 7) \mathrm{nm}$. The alumina cavity layer measures $d_{\text {cavity }}=(160 \pm 20) \mathrm{nm}$ in height. The experimentally observed reflection spectrum agrees well with the theoretical reflectivity of the structure, modelled with $n_{\mathrm{TiO}_{2}}=2.43, n_{\mathrm{Al}_{2} \mathrm{O}_{3}}=1.6, d_{\mathrm{TiO}_{2}}=30 \mathrm{~nm}$, $d_{\mathrm{Al}_{2} \mathrm{O}_{3}}=79 \mathrm{~nm}$ and $d_{\text {cavity }}=166 \mathrm{~nm}$ (Fig. 5.10b). The refractive index contrast of $\mathrm{TiO}_{2}$ and $\mathrm{Al}_{2} \mathrm{O}_{3}, \hat{n}=\frac{n_{\mathrm{TiO}_{2}}}{n_{\mathrm{Al}_{2} \mathrm{O}_{3}}} \approx 1.5$, is higher than the contrast that can be realised by using polymers and consequently the metal oxide multilayer shows a higher reflectivity in a broader wavelength range (Chapter 1 ). The resonance wavelength of the cavity structure is $\sim 425 \mathrm{~nm}$. The quality factor $Q$ of the unpatterned, flat cavity amounts to $\sim 70$.

A monolayer of $3 \mu \mathrm{m}$-sized colloids was assembled on top of the resonance cavity. Ion milling for a total exposure time of $72 \mathrm{~min}$ resulted in the multilayer cone structures displayed in Figure 5.10 e. For clarity, this structure is schematically drawn in Figure 5.10 c. Observed in an optical microscope, the sample appears blue with violet cone tips in reflection and green with yellow cone tips in transmission. Scanning electron 

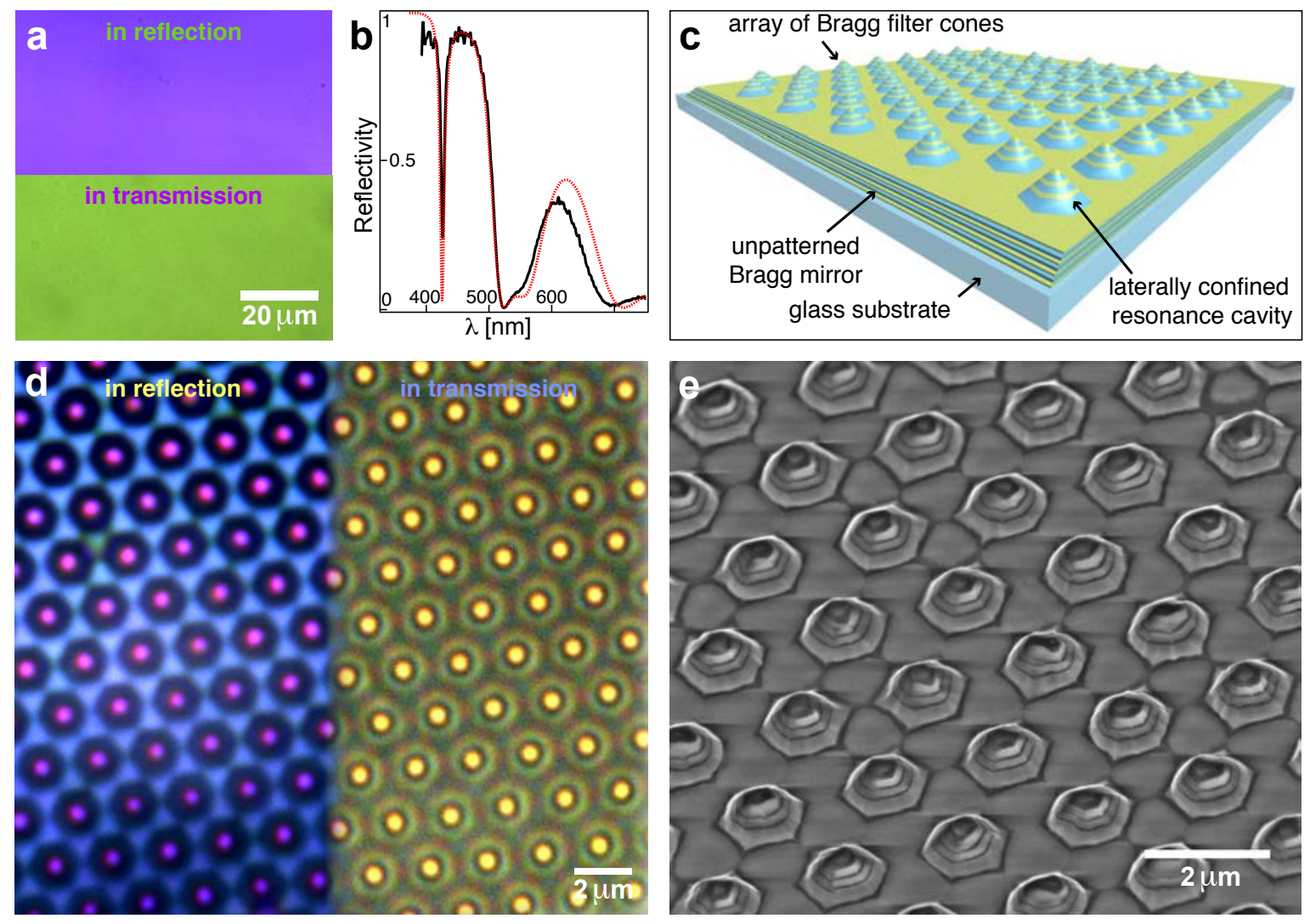

Figure 5.10: Patterning of inorganic multilayers. a) Microscopic images of the flat resonance cavity in reflection (top) and transmission (bottom) prior to the ion milling. b) Measured (black) and modelled (red) reflection spectra of the flat cavity. c) Schematic model of the multilayer cone structure after ion milling, showing the degree of patterning. d) Micrograph of the multilayer cone cavity structure in reflection (left) and transmission (right). e) SEM image of the multilayer cones. Only the top DBR and the cavity layer are structured while the bottom DBR consists of unpatterned layers.

micrograps reveal the configuration on the sample surface after ion milling (Fig. 5.10 e). Only five layers are visible in the top DBR, which indicates that the structure has been exposed to the ion beam for too long, resulting in the removal of the two top layers. Furthermore, the cavity layer and the first layer of the lower DBR are exposed in the cone structure, while the remaining six bottom layers are unstructured. The different parts of the resonance cavity cones are visualised and labelled in Figure $5.10 \mathrm{c}$.

The local reflection behaviour of the sample has been investigated with the microspectroscopic setup described in Chapter 3.4.2. Spectral maps are displayed in Figure 5.11 for the wavelengths of the cavity resonance at $416 \mathrm{~nm}(\mathrm{c})$, the reflection maxi- 

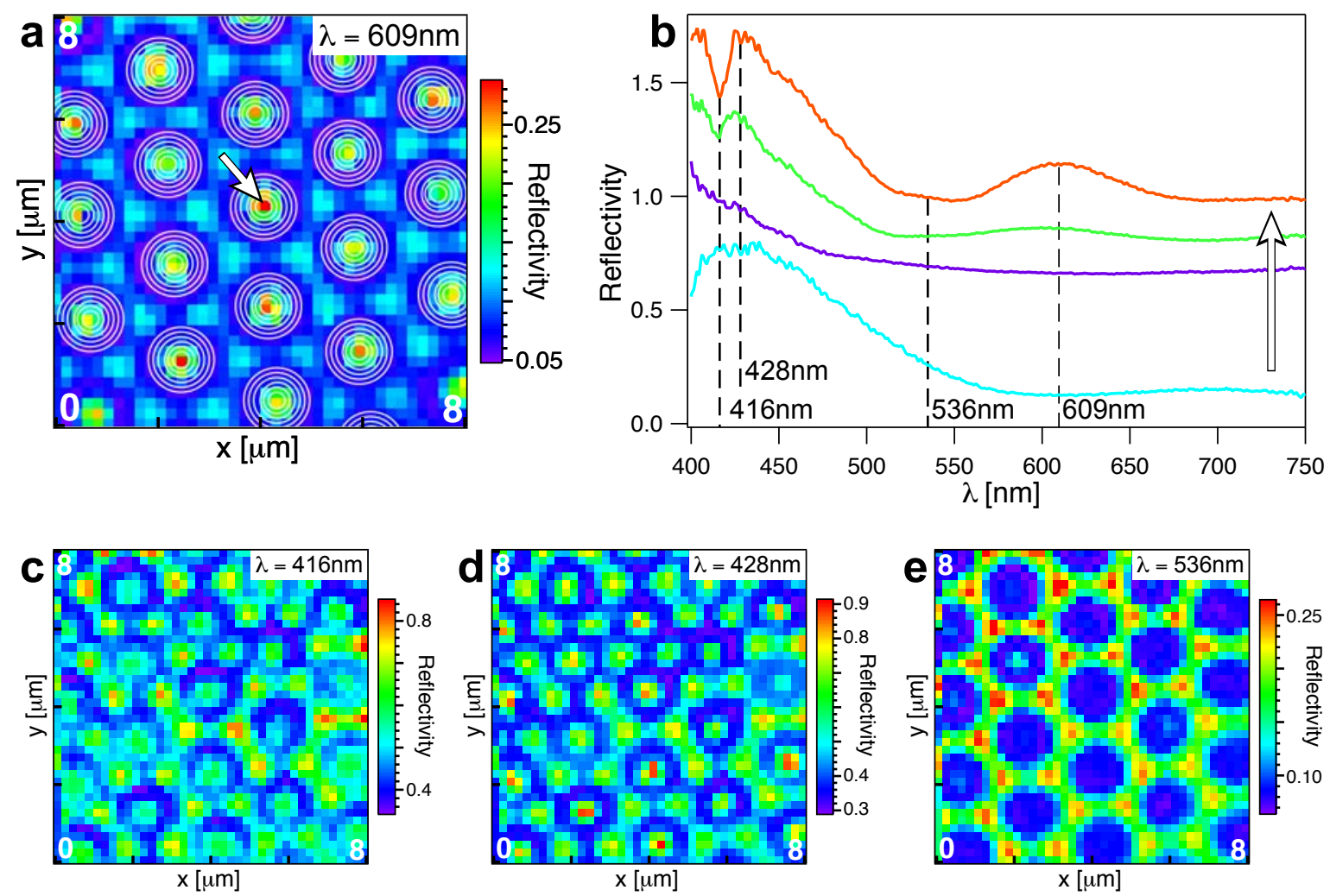

Figure 5.11: Local spectroscopic signature of the multilayer cones. a) Intensity map at $\lambda=609 \mathrm{~nm}$ with the positions of the multilayer cones shown by the white height lines. b) Reflectivity spectra acquired across one cone along the white arrow shown in (a). For clarity, the spectra are offset to each other (by 0.6 - violet, 0.75 - green, 0.9 - orange). c) - e) Reflectivity maps for $\lambda=416 \mathrm{~nm}$ (c), $\lambda=428 \mathrm{~nm}$ (d), $\lambda=536 \mathrm{~nm}(\mathbf{e})$.

mum at $428 \mathrm{~nm}(\mathrm{~d})$, the first side minimum at $536 \mathrm{~nm}(\mathrm{e})$ and the first side maximum at $609 \mathrm{~nm}$ (a), all measured at the cone centres. Increasing reflectivity is colour coded from blue to red. The corresponding wavelengths are marked in Figure 5.11 b, which shows reflectivity spectra acquired along the arrow displayed in Figure 5.11 a. For clarity, the spectra are offset from each other, the lowest stemming from the interstitial region and the topmost resulting from the centre of the cone. The positions of the cones in the spectral maps are indicated by white height lines in Figure 5.11 a. Note that the intensity scales are not the same, but shifted to display differences in intensity at particular wavelengths more clearly. The optical anisotropy of the structure is obvious in the intensity maps. Reflection spectra, shown in Figure 5.11 b, confirm the presence of resonance cavities in the centre of the cones (orange line), while they vanish 
in the interstitial regions where the structure now behaves like an ordinary DBR (light blue line). The cavity dip, registered at the cone centres, appears shallow, due to the high range of light incidence angles which is unavoidable in an optical microscope at high magnification $(\sim 160 \times)$.

The macroscopic optical behaviour of the sample is analysed with the gonio-spectrometer setup. Reflectivity and transmission measurements were performed in different geometries (SR: specular reflection, BSD: backscattering and diffraction, T: transmission, FSD: forward scattering and diffraction, described in Chapter 3.4.1) to investigate the diffraction, reflection, and transmission behaviour of the sample. The acquired data is displayed in Figure $5.12 \mathrm{a}-\mathrm{d}$. The intensities are colour coded in arbitrary units and shown as a function of wavelength and incidence angle $\theta_{\mathrm{I}}$ or detection angle $\theta_{\mathrm{D}}$. As expected, the macroscopic optical signature of the sample in specular reflection (Fig. 5.12 e) and in normal transmission (Fig. 5.12f) - shown as blue curves - differs considerably from the flat, unstructured multilayer cavity behaviour (black lines). However, the multilayer reflection peak and the corresponding transmission dip at lower wavelengths is still observable. The sample shows diffraction in reflection (Fig. 5.12 a) and transmission (Fig. $5.12 \mathrm{~b}$ ) where light that is diffracted into the $1^{\text {st }}$ and $-1^{\text {st }}$ order shows a spectral modulation caused by the multilayer interference. Specular reflection spectra (Fig. 5.12 c) and transmission spectra (Fig. 5.12d) for varying incidence angles $\theta_{\mathrm{I}}$ display features remotely similar to the ones observed from a flat multilayer, with additional complexity caused by the cone pattern. From these rich spectra, one can expect a complex dynamic colouration of the patterned multilayer surface, resulting from a combination of multilayer interference and diffraction, which depends strongly on the character of the ambient illumination, the light incidence direction and the viewer's perspective.

For the manufacture of fully separated multilayer cones, the height of the multilayer structure cannot exceed half the diameter of the colloids in the milling mask, since the aspect ratio of the cone structures is $\leq \frac{1}{2}$. Further investigations into completely separated multilayer cavity cones, using masks made from $3 \mu \mathrm{m}$ to $5 \mu \mathrm{m}$-sized colloids, are in progress. 

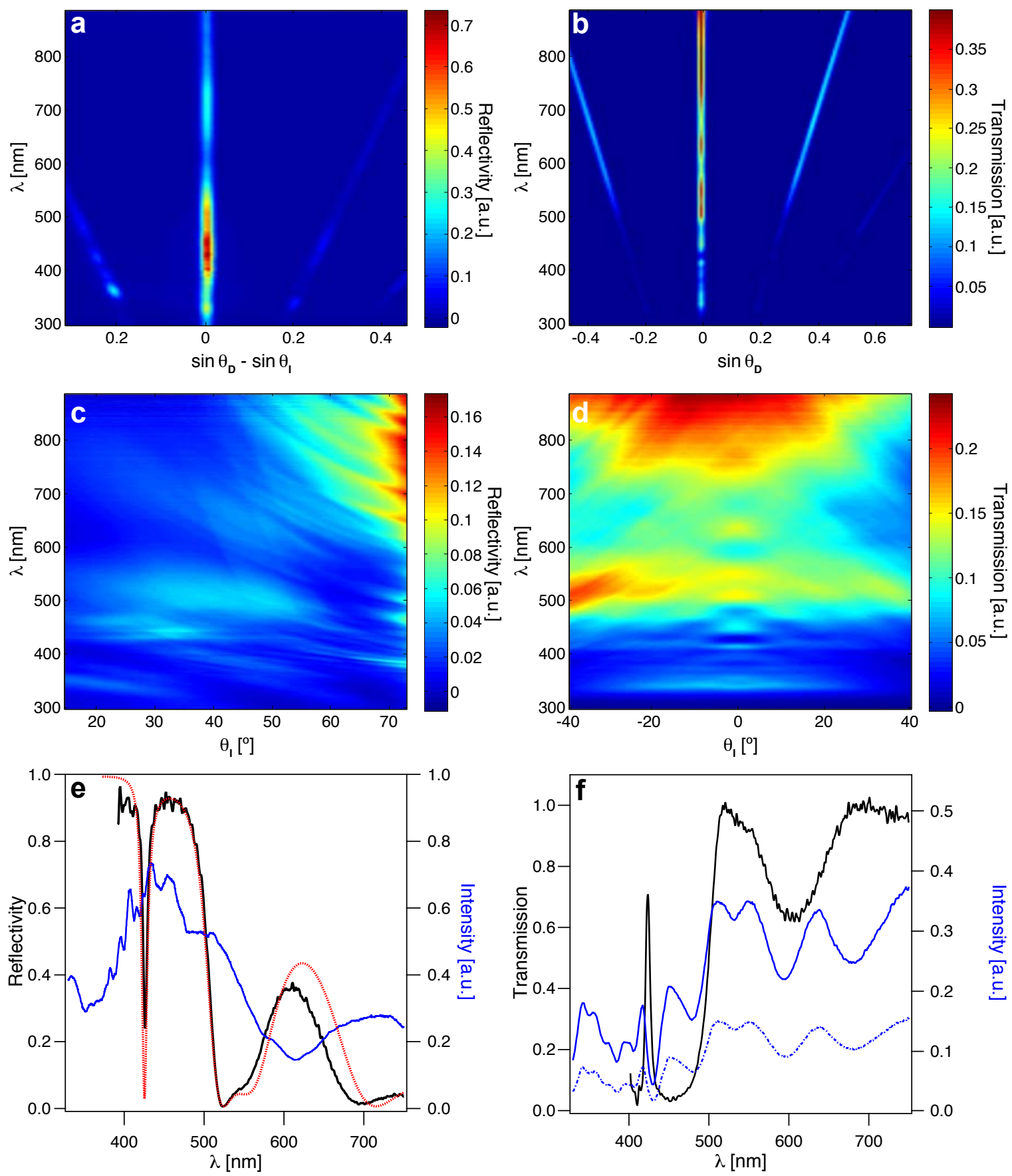

Figure 5.12: Macroscopic optical signature of the multilayer cones. a) Diffraction pattern in reflection for light incidence at an angle $\theta_{\mathrm{I}}=30^{\circ}$ (BSD-geometry). b) Diffraction pattern in transmission (FSD-geometry). c) Specular reflection as a function of incidence angle (SR-geometry). d) Direct transmission as a function of incidence angle ( $\mathrm{T}$-geometry). e) The reflectivity of the initial flat cavity structure (black), acquired from a sample area of $\sim 30 \mu \mathrm{m}$ diameter, corresponds well to theoretic predictions (red). The macroscopic specular reflection spectrum, acquired at an incidence angle of $30^{\circ}$, is shown in blue. f) Transmission of the initial flat cavity structure (black), detected from a microscopic sample area of $\sim 30 \mu \mathrm{m}$ diameter. Macroscopic transmission spectra, extracted from (b) (blue, solid line) and from (d) (blue, dashed line) at an incidence angle of $0^{\circ}$. 


\subsection{Nano-imprinting of photonic structures}

Nano-imprinting is an established technique for the creation of nanoscale surface patterns in soft materials on large areas, only limited by the size of the used imprint master. The imprinting technique is briefly described in Section 3.3.3. Results that have been obtained in attempt to imprint micro-cone structures, line gratings, and pillar arrays into poly(ethylene-co-tetrafluoroethylene) (ETFE), polystyrene films or PS-Teflon ${ }^{\circledR} \mathrm{AF}$ multilayers are presented here.

\subsubsection{Imprinting with micro-cone masters}

The silicon micro-cone structures described above can be applied as imprinting stamps with the motivation to create the inverse structures, hexagonally arranged conical holes, in polymer materials. By subsequent deposition of a thin layer of gold or silver into these hexagonally arranged cylindrical micro-holes, potential model systems for the study of plasmonic effects can be fabricated [28].

Poly(ethylene-co-tetrafluoroethylene) (ETFE) is a versatile material for imprinting. Negatives of silicon or glass patterns with feature sizes as small as $10 \mathrm{~nm}$ can be reliably transferred into thin sheets of ETFE [29]. In the pattern transfer process, the master is brought in physical contact with the ETFE sheet and the assembly is heated to $230^{\circ} \mathrm{C}$. When this temperature is reached, a pressure of 20-30 bar is applied for 500-600 s. Subsequently, with the pressure still applied, the assembly is slowly cooled down below the polymer glass transition temperature, freezing-in the imprinted structure in the ETFE sheet. One advantage of ETFE is its inherent hydrophobicity, enabling an easy release of the master once the imprinting procedure is completed.

At a pressure of 30 bar, applied for $500 \mathrm{~s}$ at $230^{\circ} \mathrm{C}$, negatives of silicon cone masters with different aspect ratios were transferred with high fidelity into ETFE. Using the ETFE patterns in a second imprinting step, positive replica of the silicon structures were created in thin polystyrene films at a pressure of 20 bar applied for $500 \mathrm{~s}$ at $120^{\circ} \mathrm{C}$. In a different approach, negatives of the cone structures were reliably produced by casting 

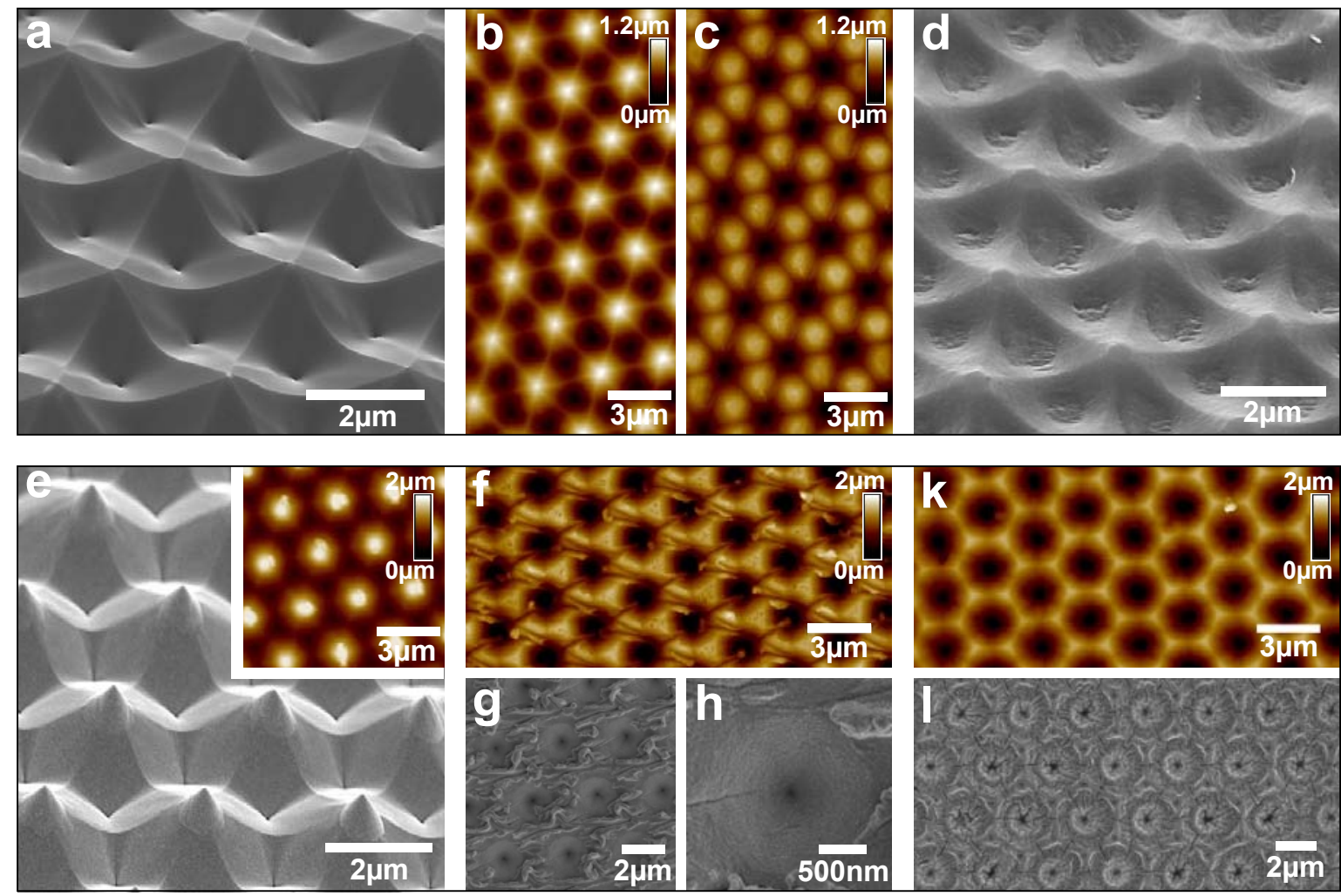

Figure 5.13: Imprinting with silicon micro-cone structures. a, Silicon microcones, aspect ratio $\approx \frac{1}{3}$. b. AFM image of the silicon master. c, AFM image of the ETFE imprint. d, Cone pattern in a polystyrene film, transferred via imprinting with the ETFE negative. e, Silicon micro-cones, aspect ratio $\approx \frac{1}{2}$, the inset shows an AFM image of the cones. $\mathbf{f}, \mathbf{g}, \mathbf{h}$, AFM and SEM images of an ETFE negative imprinted with the master in (e). Small grooves across the sample result from the tips of the master scratching over the imprint during release. The ETFE sheet has been coated with a $50 \mathrm{~nm}$ gold film prior to the acquisition of the SEM images. $k$, AFM image of a PDMS cast of the silicon cones in (e). I, SEM image of the patterned PDMS film with a gold coating of $50 \mathrm{~nm}$.

polydimethylsiloxane (PDMS) onto the silicon master. Figure 5.13 shows two microcone imprinting masters with a periodicity of $3 \mu \mathrm{m}$, and aspect ratios $\frac{1}{3}(\mathrm{a}, \mathrm{b})$ and $\frac{1}{2}(\mathrm{e})$, together with the fabricated ETFE negatives $(b, f)$. In addition, a positive PS replica of the first master is shown in (d) and a PDMS cast, taken directly from the second master is displayed in $(k, l)$. The SEM image of the polystyrene replica (d) reveals that the initial polymer film was slightly too thin, resulting in an incomplete filling of the ETFE structure during imprinting, consequently preventing optimum replication of the structure. Care has to be taken during release of the polymer film from the imprint master, otherwise the cone tips leave scratches in the patterns, which can be 
seen in the AFM and SEM pictures of an ETFE imprint in Figures $5.13 \mathrm{f}, \mathrm{g}, \mathrm{h}$.

After deposition of a $50 \mathrm{~nm}$ thick gold film, the ETFE negatives display some surface roughness (Fig. $5.13 \mathrm{~g}, \mathrm{~h}$ ). In strong contrast, the PDMS structures have changed drastically after gold deposition, displaying distinct wrinkles and cracks (Fig. 5.13 l), which render these surfaces unusable for the systematic study of plasmonic effects. The roughness of the ETFE films also induces scattering of light, which complicates the study of surface plasmons, that can potentially be observed in the conical hole structures. So far, reproducible plasmonic features have been detected on neither the gold coated PDMS nor on the ETFE surfaces. More effort has to be put into the fine-tuning of the imprinting or casting processes to create smooth walled conical holes. The smoothness of the replica is ultimately limited by the surface roughness of the silicon micro-cones which also has to be investigated further.

\subsubsection{Replication of micro-cones for flexible cell substrates}

Even though the micro-cone imprinting process has so far proven to be unsuitable for plasmonic applications, it might be interesting for other purposes. In a two-step imprinting and casting process positive replica of the cones can be produced into PDMS sheets for the use as cell-growth and cell-patterning substrates [30]. First, the microcone master is imprinted into ETFE as described above. Here, silicon micro-cones with flat tops are used to provide platforms for better cell adhesion (Fig.5.14 a). Second, PDMS is cast onto the ETFE imprints (Fig. 5.14 b) and cured at $60^{\circ} \mathrm{C}$ for several hours. After the lift-off of the PDMS cast from the ETFE a positive PDMS replica of the cones is revealed (Fig. $5.14 \mathrm{c}, \mathrm{d}$ ). Preliminary cell culturing experiments performed by Ivan Minev (Nanoscience Centre, University of Cambridge) with fibroblast cells on these surfaces suggest that the cells extend into specific directions following the lattice directions of the two dimensional cone crystal underneath the cell (Fig. 5.14 e). 

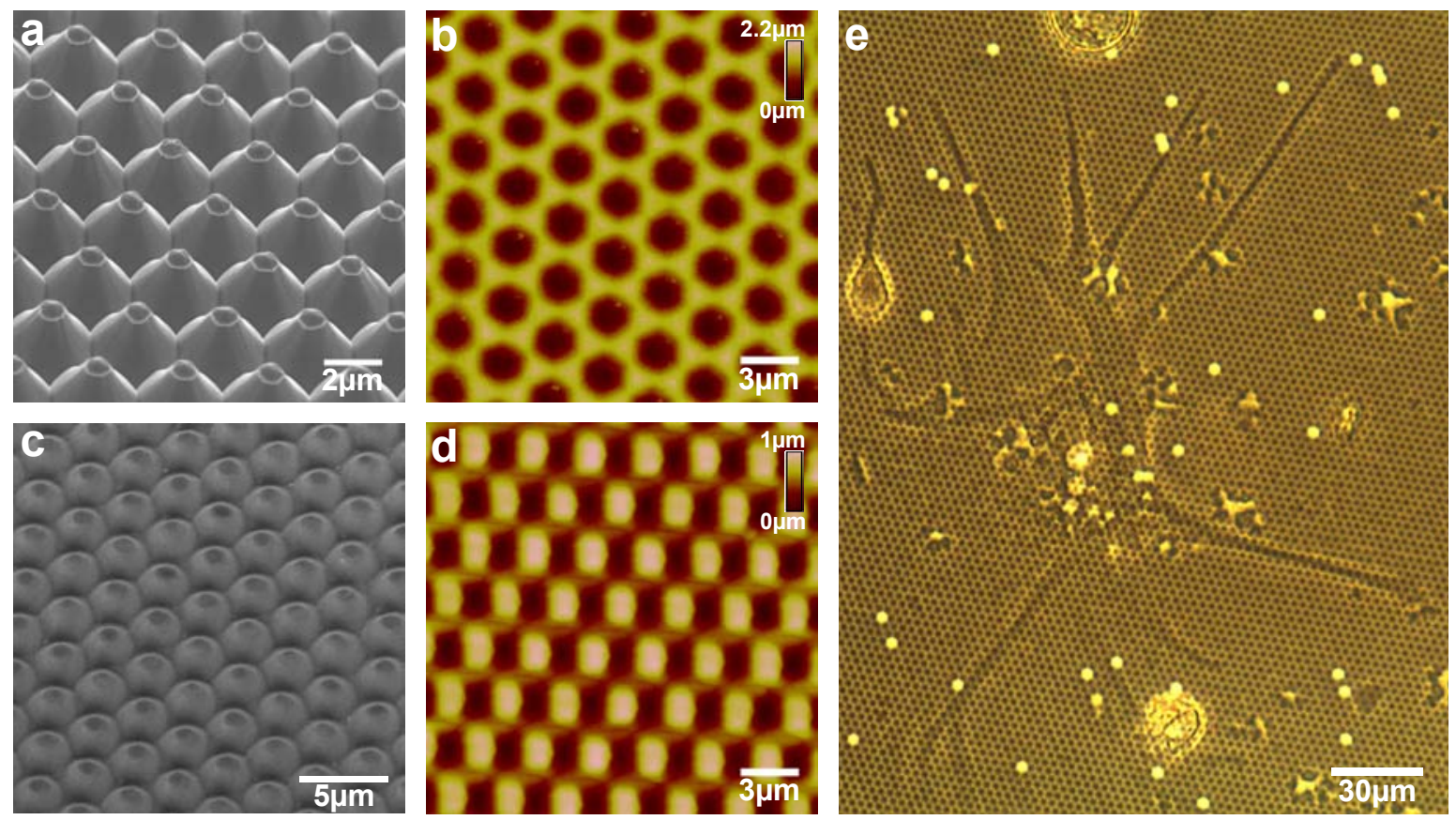

Figure 5.14: PDMS micro-cone replica used as cell templates. a) Micro-cone master before removal of the polymer caps. b) AFM image of the ETFE sheet after imprinting. c) Positive PDMS replica of the micro-cones, produced by casting of the ETFE mold. d) AFM image of the PDMS replica. e) A fibroblast cell spread on the patterned PDMS surface. The cell seems to extend following certain orientations of the crystal lattice.

\subsubsection{Fabrication of an imprint master for the replication of butterfly wing scale patterns}

Convex or concavely shaped micro-structures pose several challenges to conventional pattern transfer processes. Nano-lithography offers a versatile alternative to the transfer of concave and convex micro - or nano-patterns, provided an appropriate master is available. Here, a possible route for the fabrication of an imprinting master that allows the replication of concavities found on the wing scales of the butterfly Papilio blumei is described briefly and first pattern transfer results are presented.

For the construction of the imprint master, a monolayer of hexagonally arranged, close packed colloids is deposited onto glass or silicon. An inverse of the colloids is then created by imprinting the colloidal layer into dental wax, which cures within 10 min (Section 3.3.4). Epoxy is cast into the dental wax mold and cured at $60^{\circ} \mathrm{C}$ for several 

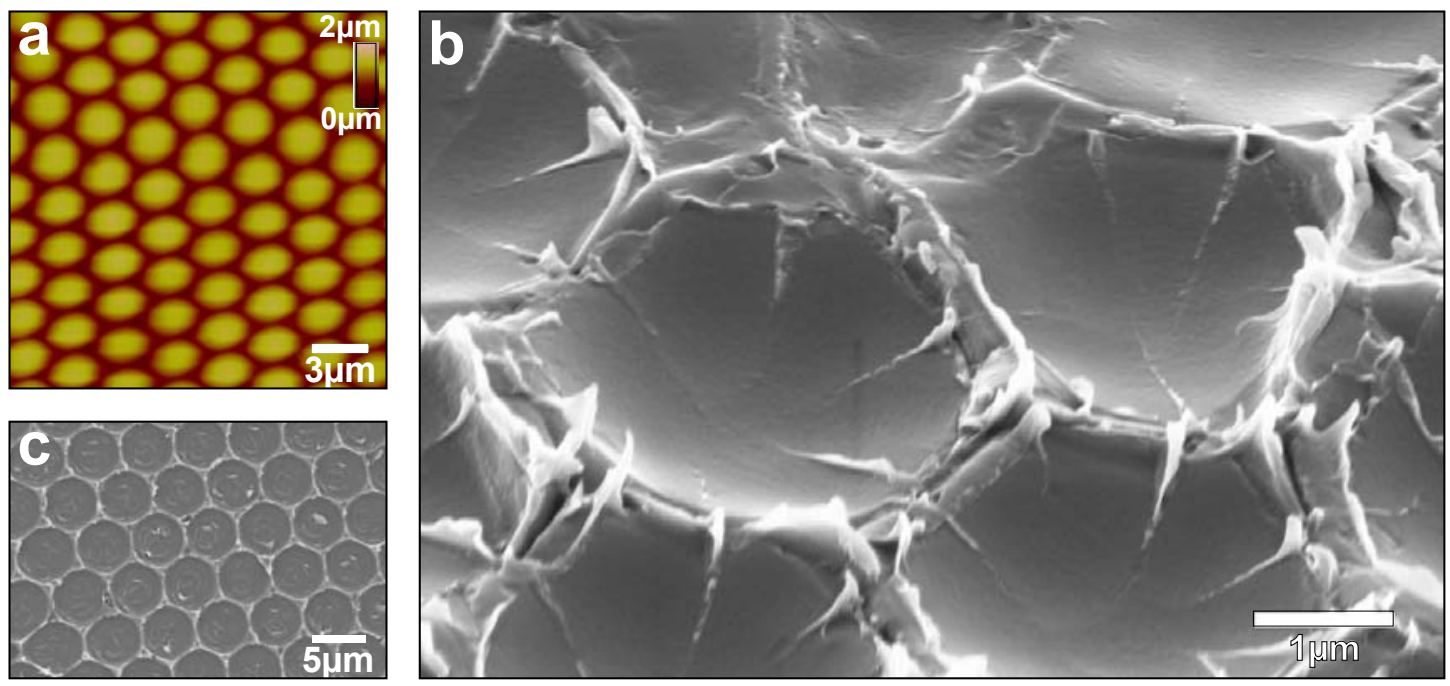

Figure 5.15: Imprinting of butterfly wing scale concavities. a) Epoxy master with regularly arranged, $3 \mu \mathrm{m}$-sized, half spherical bumps. b) Imprinted polystyrene film imaged at $45^{\circ}$ angle. c) Polystyrene film in top view, after imprinting of $5 \mu \mathrm{m}$-sized concavities.

hours. The resulting structure is a positive replica of the top profile of the initial colloidal pattern (Fig. 5.15a).

Before being employed in the imprinting process, the epoxy surface is coated with a $\sim 20 \mathrm{~nm}$ thick layer of $\mathrm{SiO}_{2}$ by sputter coating and subsequently rendered apolar in a silanisation step, by exposing it in a desiccator to an atmosphere of $1 \mathrm{H}, 1 \mathrm{H}, 2 \mathrm{H}, 2 \mathrm{H}-$ perfluorodecyltrichlorosilane for four hours.

Imprinting of the master into polystyrene films is done with a pressure of $30 \mathrm{bar}$ for $400 \mathrm{~s}$ at $120^{\circ} \mathrm{C}$. Different perspectives of prototype imprints are shown in Figure $5.15 \mathrm{~b}, \mathrm{c}$. The concavities are locally imprinted into the polystyrene films but show a considerable amount of defects, possibly arising from residual sticking of the polymer to the master in the narrower interstitial regions. Consequently, the protocol used for the passivation of the master needs to be improved. In addition, the epoxy masters show some signs of deformation during the imprinting. The use of high temperature stable epoxy (EpoTek OE132) would prevent the deformation of the stamp. However, a considerable shrinkage of the epoxy during curing makes the production of the stamp more difficult. Although first results of stamp creation and pattern replication are promising, this route needs to be investigated and optimised further. 


\subsubsection{Multilayer patterning by nano-imprinting}

Preliminary results on the the suitability of the nano-imprinting technique for pattern transfer into PS-Teflon ${ }^{\circledR} \mathrm{AF}$ multilayer films are presented here. ETFE negatives of silicon micro-cones, silicon line patterns and arrays of cylinders on silicon were used as imprinting masters.

While the glass transition of polystyrene happens at $\sim 100^{\circ} \mathrm{C}[31]$, the transition temperature of the amorphous Teflon component lies at $T_{\mathrm{g}} \approx 160^{\circ} \mathrm{C}$. In order to transfer a pattern into both materials, the imprinting temperature has to be higher than the $T_{\mathrm{g}}$ of Teflon ${ }^{\circledR} \mathrm{AF}$.

However, imprinting at temperatures higher than $170^{\circ} \mathrm{C}$ induces partial dewetting of polystyrene on the Teflon ${ }^{\circledR} \mathrm{AF}$ layers, sometimes resulting in quite pretty structures (Fig. 5.16). Furthermore, the stability of ETFE masters is not assured at temperatures much higher than $170^{\circ} \mathrm{C}$ [29]. To prevent dewetting and to conserve the structure in the ETFE masters, the following structures were imprinted at temperatures below $170^{\circ} \mathrm{C}$.

Line patterns with a periodicity of $1.5 \mu \mathrm{m}$ and feature height of $200 \mathrm{~nm}$ were reliably replicated only in the topmost polystyrene layer of a PS-Teflon ${ }^{\circledR} \mathrm{AF}$ multilayer stack (Fig. 5.17), while the underlying layers remained unmodified by the imprinting, pre-
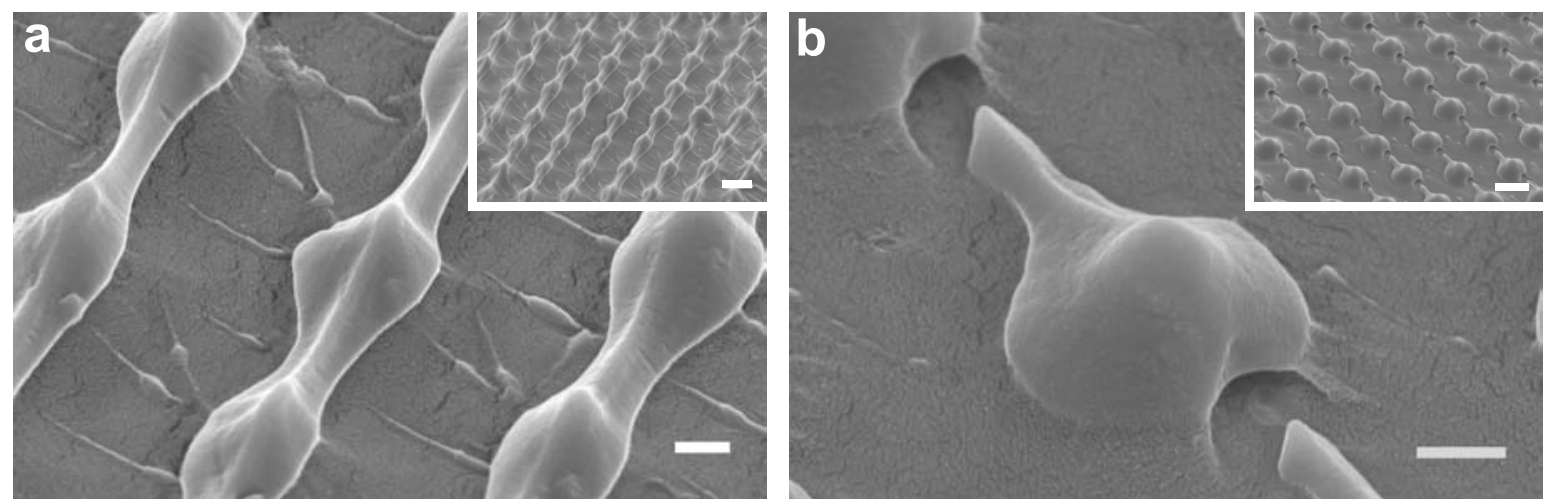

Figure 5.16: Imprinting induced dewetting. Imprinting of an ETFE master with hexagonally arranged cylindrical holes into a PS-Teflon ${ }^{\circledR} \mathrm{AF}$ multilayer was performed at a pressure of $30 \mathrm{bar}$ at $175^{\circ} \mathrm{C}$ for $300 \mathrm{~s}$. Partial dewetting of the top polystyrene layer during the imprinting process results in strangely shaped, periodic patterns. Scale bars a) $500 \mathrm{~nm}$, inset $2 \mu \mathrm{m}$, b) $500 \mathrm{~nm}$, inset $1 \mu \mathrm{m}$. 

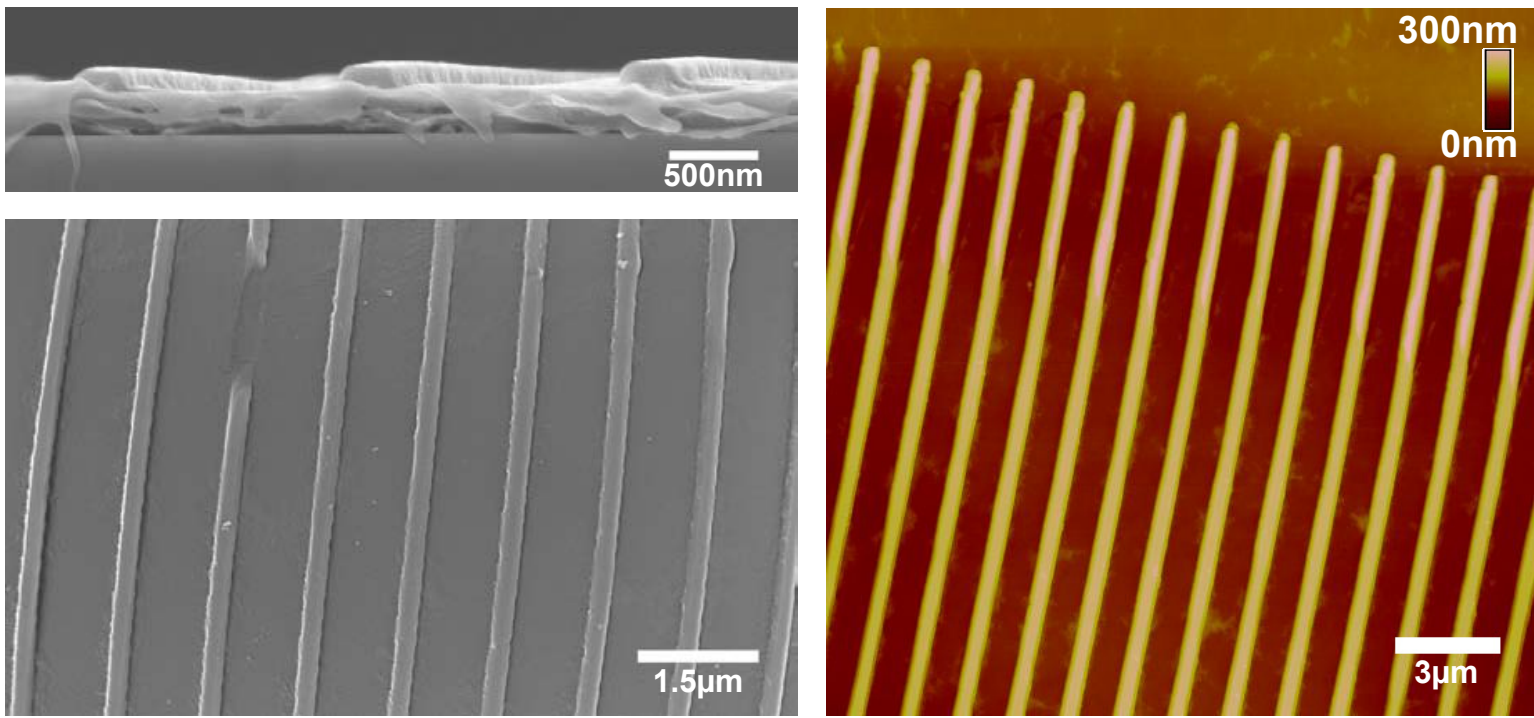

Figure 5.17: Imprinting of line patterns. a) Cross-section of a patterned multilayer stack with five alternating polystyrene and Teflon ${ }^{\circledR}$ AF films. b) Top view of the patterned stack. c) AFM image of the patterned multilayer.

sumably due to the structural support offered by the glassy Teflon ${ }^{\circledR}$ AF layers.

In imprinting experiments with $800 \mathrm{~nm}$ high pillar structures arranged on a square grid of $8 \mu \mathrm{m}$ periodicity (Fig. 5.18), a similar behaviour was observed. While only the first polystyrene layer was reliably structured, often dewetting of this polystyrene layer occurred on top of the underlying Teflon ${ }^{\circledR}$ AF surface (Fig. 5.18 b). Optical micrographs of a patterned multilayer on silicon with three alternating polystyrene and Teflon ${ }^{\circledR} \mathrm{AF}$ films of thickness $d_{\mathrm{PS}} \approx 80 \mathrm{~nm}$ and $d_{\mathrm{TAF}} \approx 100 \mathrm{~nm}$ (Fig. $5.18 \mathrm{c}$ ) and a patterned stack of eleven alternating layers on glass show the inhomogeneities of the film around and on the pillars, which arise from dewetting. The insets show a larger section of the patterned film visualising the optical inhomogeneities that arise from the inherent waviness of the Teflon ${ }^{\circledR}$ AF films, which was discussed in Section 4.1.1.

In summary, the reliable and complete patterning of polystyrene-Teflon ${ }^{\circledR} \mathrm{AF}$ multilayers by nano-imprinting has not been achieved within the context of this work. In order to establish this procedure and to successfully transfer a pattern across a complete multilayer stack, systematic investigations of the imprinting parameters, suitable master structures and the imprinting-induced dewetting phenomenon are necessary. 

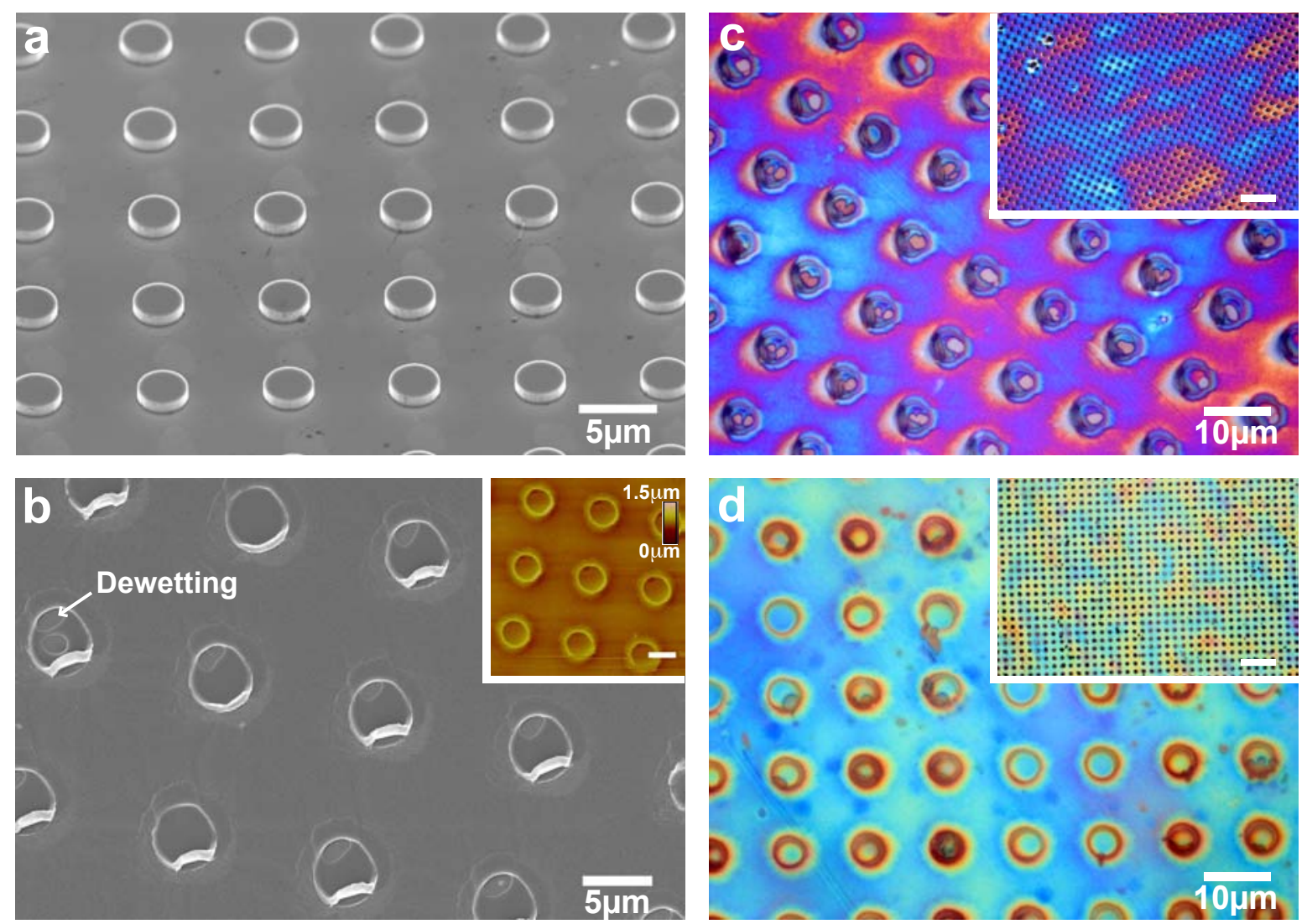

Figure 5.18: Imprinting of pillar structures in polymer multilayers. a) SEM image of a silicon pillar master. b) Image of an imprint in a multilayer of three alternating PS and Teflon ${ }^{\circledR}$ AF films on silicon. The inset shows an AFM scan of the surface, scale bar $5 \mu \mathrm{m}$. c) Microscope image of the sample in (b). The inset shows a larger area of the surface, scale bar $50 \mu \mathrm{m}$. d) Microscope image of a multilayer with 11 alternating PS and Teflon ${ }^{\circledR}$ AF films on glass. The inset shows a larger area of the surface, scale bar $50 \mu \mathrm{m}$.

\subsection{Atomic layer deposition on structured substrates}

The fabrication of structured multilayers is considerably facilitated by the use of atomic layer deposition. The concept of coating any arbitrarily shaped surface with a uniform metal oxide film is easily expanded to apply multilayers of titania and alumina with high surface conformity onto pre-structured surfaces. Glass micro-cone structures of low aspect ratio with a periodicity of $2 \mu \mathrm{m}$, produced by template-assisted ion milling, were used as the template. A resonance cavity structure, consisting of two Bragg mirrors with nine alternating titania and alumina layers enclosing an alumina cavity layer, was deposited at $200^{\circ} \mathrm{C}$. The thickness of the titania and alumina layers were 

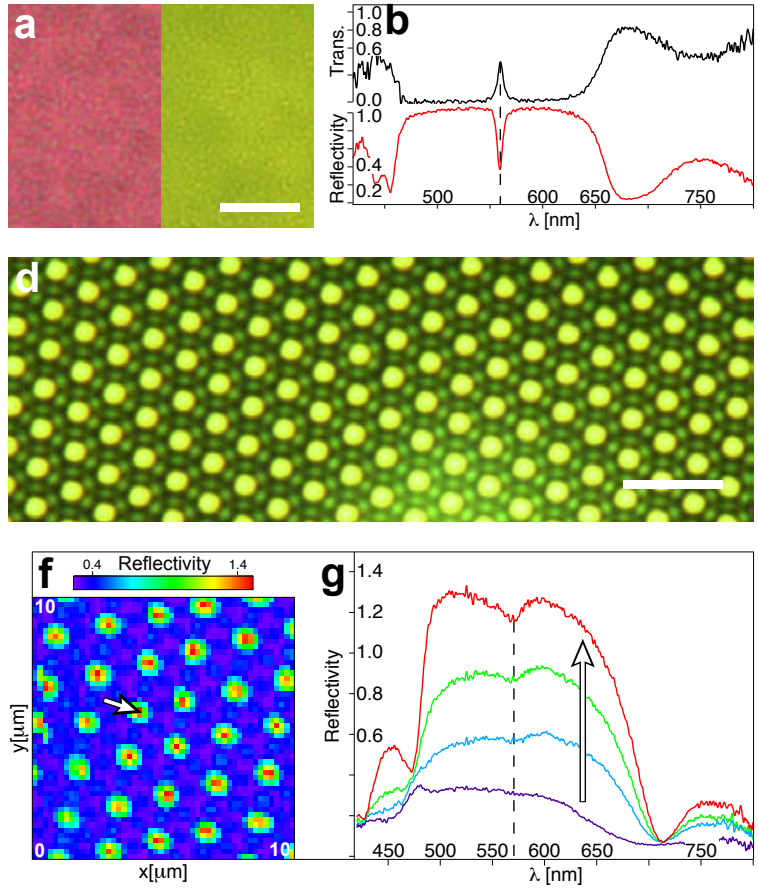
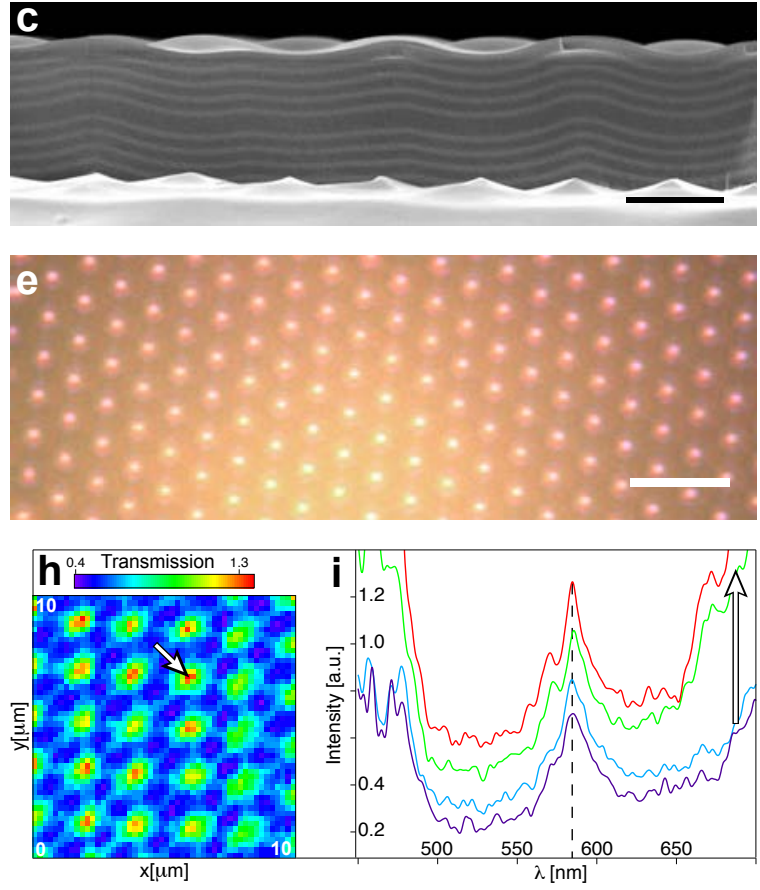

Figure 5.19: Conformal deposition of a multilayer on structured surfaces. a) Appearance of a reference sample with a flat $\mathrm{TiO}_{2}-\mathrm{Al}_{2} \mathrm{O}_{3}$ multilayer resonance cavity on glass in transmission (pink) and in reflection (green), scale bar $20 \mu \mathrm{m}$. b) Transmission (black) and reflection (red) spectra of the flat resonance cavity. c) Cross-section of a $\mathrm{TiO}_{2}-\mathrm{Al}_{2} \mathrm{O}_{3}$ micro-cavity, deposited by $\mathrm{ALD}$ onto a low aspect ratio micro-cone structured glass surface, scale bar $1 \mu \mathrm{m}$. d) Structured multilayer resonance cavity in reflection and e) in transmission, scale bars $5 \mu \mathrm{m}$. f) Reflectivity map. g) Reflectivity spectra that were acquired along the path indicated by the white arrow in the reflectivity map (f). Colours of the spectra correspond to colours in the spectral map. The resonance dip in the interstitial area has disappeared due to the concave shape of the multilayer cavity and the high angular range of light incidence directions. h) Transmission map. i) Transmission spectra that were acquired along the path indicated by the white arrow in the transmission map (h). For clarity, spectra are offset along the vertical axis by 0.1 .

$d_{\mathrm{TiO}_{2}}=(56 \pm 2) \mathrm{nm}, d_{\mathrm{Al}_{2} \mathrm{O}_{3}}=(85 \pm 2) \mathrm{nm}$. The alumina cavity layer had double the thickness of the alumina layers in the Bragg mirrors. Figure 5.19 a shows the optical appearance of the flat resonance cavity in transmission and reflection. Corresponding transmission and reflection spectra are displayed in Figure 5.19 b. The resonance cavity had a stop bandwith of $196 \mathrm{~nm}$ and a quality factor $Q \approx 97$.

A cross-section through the cavity is shown in Figure $5.19 \mathrm{c}$. The individual layers follow the substrate structure with a high fidelity. Optical micrographs (Fig. 5.19d,e) visualise the optical anisotropy which can also be analysed spectroscopically. Intensity 
maps acquired at high magnification from a $\sim 100 \mu^{2}$-sized sample region at normal incidence in reflection and transmission (Fig. $5.19 \mathrm{f}, \mathrm{h}$ ) and the corresponding spectra (Fig. $5.19 \mathrm{~g}, \mathrm{i}$ ) reveal the microscopic optical properties of the sample. In reflection the resonance dip is still observable at the centres of the cones but vanishes in the interstitial regions. The observed resonance dip in the reflection spectra is very shallow. This behaviour is expected, due to the dependence of the resonance wavelength on the angle of light incidence combined with the fact that the measurements at high magnification are done using a lens with very short focal length, which implies a large range of angles at which light impinges on the sample surface. In transmission, the cavity peak is present in the interstitial regions and appears to be slightly more pronounced with a smaller peak width in the cone centres. This peak width is considerably larger compared to the peak width of the flat resonance cavity. The spectral broadening is presumably due to the local orientational variation of the multilayer surface normal with respect to the incident light. The local angle of light incidence varies from $-15^{\circ}$ to $+15^{\circ}$ with respect to the global surface normal in an area of only $4 \mu^{2}$ and the centre wavelength of the transmission peak changes accordingly. Consequently, the broadening of the transmission peak in the spectra acquired from a surface area of $\sim 1 \mathrm{\mu m}^{2}$ contain information about the slight variation in optical path length and interference condition that the transmitted light incurs on this length scale.

\subsection{Conclusion}

Low aspect ratio, micro-patterns can be etched into organic multilayer structures by template-assisted ion milling. For organic structures with higher aspect ratios, masking materials with better ion milling resistance have to be found. Further improvements could presumably make this technique a promising alternative for the creation of patterns in all-polymer one-dimensional photonic crystals on large sample areas. Quasi three-dimensional tunable photonic crystals produced by template-assisted ion milling from elastic, flexible polymer multilayers (Chapter 4) can be potential building blocks for novel optical devices. 
Early stage results suggest that nano-imprint lithography offers several direct or indirect routes to produce complex patterns for optical elements but further effort has to be made to improve the presented approaches.

Metal oxides are widely used in industry for the production of optical devices, due to their good optical properties, which make them interesting materials for the creation of complex photonic structures. In preliminary experiments, static metal oxide multilayer micro-cone structures have been fabricated by template-assisted ion milling and surface conformal metal oxide multilayers have been deposited by atomic layer deposition on patterned substrates. In particular, atomic layer deposition of conformal multilayers on non-planar substrates was applied to create artificial replicas of the Papilio blumei wing scale structure. This is described in detail in the following chapter. 



\section{References}

[1] Vieu, C. et al. Electron beam lithography: Resolution limits and applications. Appl. Surf. Sci. 164, 111-117 (2000).

[2] Watt, F., Bettiol, A., Kan, J. V., Teo, E. \& Breese, M. Ion beam lithography and nanofabrication: a review. Int. J. Nanosci. Ser. 4, 269-286 (2005).

[3] Melngailis, J. Focused ion beam technology and applications. J. Vac. Sci. Technol. $B$ 5, 469 (1987).

[4] Melngailis, J., Mondelli, A., Berry, I. \& Mohondro, R. A review of ion projection lithography. J. Vac. Sci. Technol. B 16, 927 (1998).

[5] Deubel, M. et al. Direct laser writing of three-dimensional photonic-crystal templates for telecommunications. Nature Mater. 3, 444-447 (2004).

[6] Wong, S. et al. Direct laser writing of three-dimensional photonic crystals with a complete photonic bandgap in chalcogenide glasses. Adv. Mater. 18, 265-269 (2006).

[7] Ito, R. \& Okazaki, S. Pushing the limits of lithography. Nature 406, 1027-1031 $(2000)$.

[8] Perry, J. Two beams squeeze feature sizes in optical lithography. Science 324, 892 (2009).

[9] Scott, T., Kowalski, B., Sullivan, A., Bowman, C. \& McLeod, R. Two-color singlephoton photoinitiation and photoinhibition for subdiffraction photolithography. Science 324, 913 (2009).

[10] Li, L., Gattass, R., Gershgoren, E., Hwang, H. \& Fourkas, J. Achieving $\lambda / 20$ 
resolution by one-color initiation and deactivation of polymerization. Science $\mathbf{3 2 4}$, $910(2009)$.

[11] Wagner, C. \& Harned, N. EUV lithography: Lithography gets extreme. Nature Phot. 4, 24-26 (2010).

[12] Hoffnagle, J., Hinsberg, W., Sanchez, M. \& Houle, F. Liquid immersion deepultraviolet interferometric lithography. J. Vac. Sci. Technol. B 17, 3306 (1999).

[13] Scherer, M. R. Nanostructured Materials via Self-Assembled Templates. Master's thesis, University of Cambridge (2009).

[14] Williams, K., Gupta, K. \& Wasilik, M. Etch rates for micromachining processingpart II. J. Microelectromech. S. 12, 761-778 (2003).

[15] Commonwealth Scientific Corporation. Ion beam etch rates. Bulletin \# 137-78.

[16] Braun, P. \& Wiltzius, P. Macroporous materials - electrochemically grown photonic crystals. Curr. Opin. Colloid In. 7, 116123 (2002).

[17] Abdelsalam, M., Bartlett, P., Baumberg, J. \& Coyle, S. Preparation of arrays of isolated spherical cavities by self-assembly of polystyrene spheres on self-assembled pre-patterned macroporous films. Adv. Mater. 16, 90-93 (2004).

[18] Bartlett, P., Baumberg, J., Coyle, S. \& Abdelsalam, M. Optical properties of nanostructured metal films. Faraday Discuss. 125, 117-132 (2004).

[19] Mahajan, S. et al. Tuning plasmons on nano-structured substrates for NIR-SERS. Phys. Chem. Chem. Phys. 9, 104-109 (2007).

[20] Tian, Z. Surface-enhanced Raman spectroscopy: Advancements and applications. J. Raman Spectrosc. 36, 466-470 (2005).

[21] Kneipp, K. et al. Single molecule detection using surface-enhanced Raman scattering (SERS). Phys. Rev. Lett. 78, 1667-1670 (1997).

[22] Nie, S. \& Emory, S. Probing single molecules and single nanoparticles by surfaceenhanced Raman scattering. Science 275, 1102 (1997). 
[23] Crossland, E. J. Block Copolymer Patterning of Functional Materials. Ph.D. thesis, University of Cambridge (2008).

[24] Smit, M., Acket, G. \& van der Laan, C. $\mathrm{Al}_{2} \mathrm{O}_{3}$ films for integrated optics. Thin solid films 138, 171-181 (1986).

[25] Bagnall, D. et al. Optically pumped lasing of $\mathrm{ZnO}$ at room temperature. Appl. Phys. Lett. 70, 2230 (1997).

[26] Chen, K. et al. $\mathrm{SiO}_{2} / \mathrm{TiO}_{2}$ omnidirectional reflector and microcavity resonator via the sol-gel method. Appl. Phys. Lett. 75, 3805 (1999).

[27] Boudaden, J., Oelhafen, P., Schüler, A., Roecker, C. \& Scartezzini, J. Multilayered $\mathrm{Al}_{2} \mathrm{O}_{3} / \mathrm{SiO}_{2}$ and $\mathrm{TiO}_{2} / \mathrm{SiO}_{2}$ coatings for glazed colored solar thermal collectors. Sol. Energ. Mat. Sol. C. 89, 209-218 (2005).

[28] Perney, N. et al. Tuning localized plasmon cavities for optimized surface-enhanced Raman scattering. Phys. Rev. B 76, 35426 (2007).

[29] Barbero, D. R. et al. High resolution nanoimprinting with a robust and reusable polymer mold. Adv. Funct. Mater 17, 2419-2425 (2007).

[30] Dowell-Mesfin, N. et al. Topographically modified surfaces affect orientation and growth of hippocampal neurons. J. Neural Eng. 1, 78 (2004).

[31] Bandrup, J., Immergut, E. H. \& Grulke, E. A. (eds.) Polymer handbook (John Wiley \& Sons, 1999), $4^{\text {th }}$ edn. 



\section{Chapter $\mathbf{6}$}

\section{Mimicry of Papilio blumei's colourful wing scale structure}

The brightest and most vivid natural colours arise from the interaction of light with periodic micro- or nanostructures. While the physics of structural colours is well understood, it remains a challenge to create artificial replicas of natural photonic structures [1-3]. In this chapter we use a self-organisation process for the efficient and scaleable fabrication of photonic structures, which mimic the colour mixing effect found on the wings of the Indonesian butterfly Papilio blumei. Furthermore, we show that a conceptual variation to the natural structure leads to enhanced optical properties. This significantly extends earlier attempts [4-6], which were limited in terms of versatilely and scalability.

Brilliant, saturated colours in nature arise from multilayer interference, optical gratings, photonic crystals, and micro-optical elements. Particularly in butterflies, several of these strategies are combined to give rise to complex colour mixing. The intricate structures on butterfly wing scales are difficult to copy and the mimicry of the colour mixing effect on the wing scales of the butterflies Papilio blumei and Papilio palinurus $[7,8]$ is particularly challenging. The Papilio wing scale structure consists of a regularly deformed multilayer stack made from alternating cuticle and air layers creating intense structural colours (Fig. 6.1). Although the Papilio blumei wing scales were used as a template for atomic layer deposition [9], such an approach is not suitable for accurate replication of the internal multilayer structure on large surface areas. 


\subsection{The role model}

The bright green coloured areas on Papilio blumei and Papilio palinurus wings result from a juxtaposition of blue and yellow-green light reflected from different microscopic regions on the wing scales. Light microscopy reveals that these regions are the centres (yellow) and edges (blue) of $5-10 \mu \mathrm{m}$ wide concavities, clad with a perforated cuticle
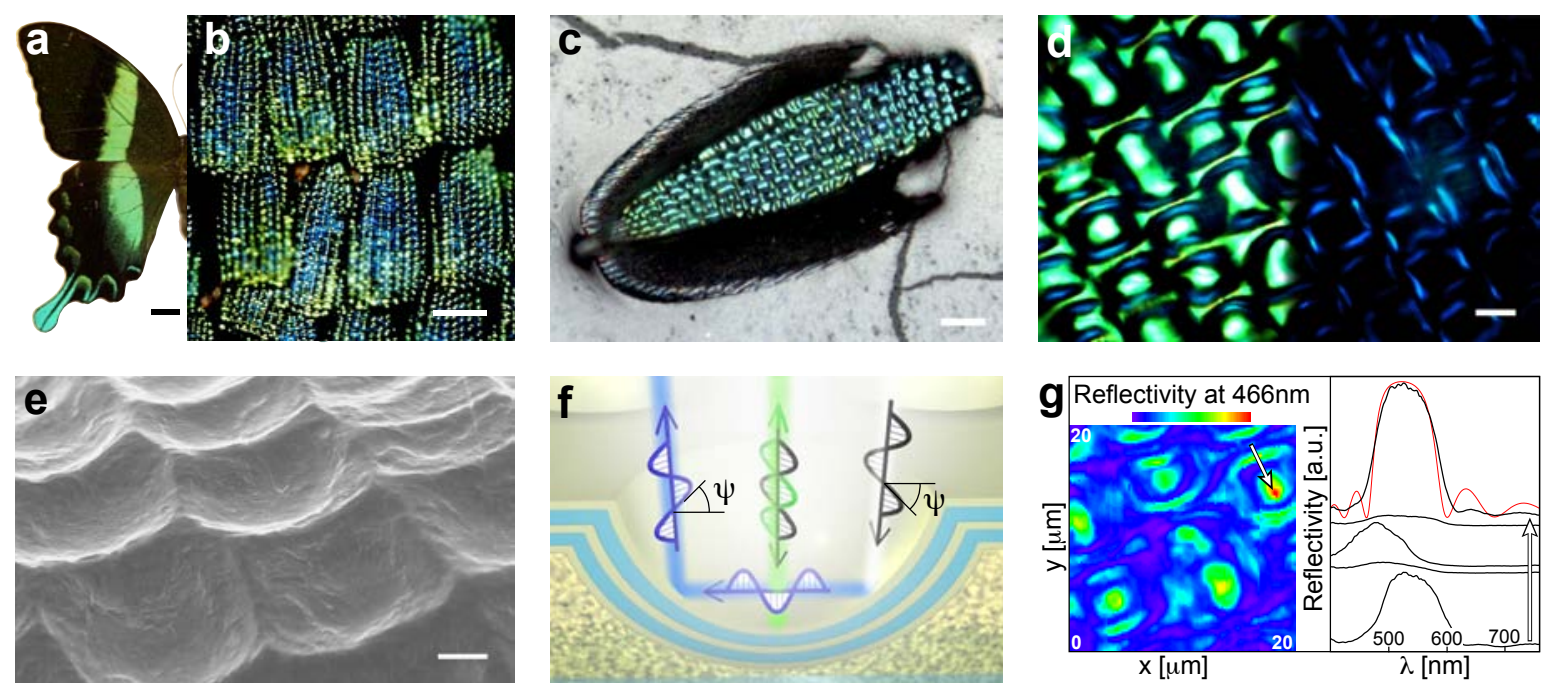

Figure 6.1: Natural photonic structure. The bright green on Papilio blumei's wings (a, scale bar $1 \mathrm{~cm}$ ) results from a colour mixing [7] of blue and yellow-green light reflected from different wing scale regions (b, scale bar $100 \mu \mathrm{m}$ ). Papilio blumei has two types of scales: The first kind provides the colour while the second kind, lying under the first, absorbs the transmitted light, preventing it from being backscattered, thus assuring the purity of the reflected colours (c, scale bar $20 \mu \mathrm{m}$ ). The surface of a reflecting wing scale (optical micrograph in (d) and scanning electron micrograph in (e), scale bars $5 \mu \mathrm{m}$ and $2 \mu \mathrm{m}$ ) is covered with concavities of about $5-10 \mu \mathrm{m}$ diameter arranged in ordered lines along the scale. These concavities are clad with a multilayer reflecting yellow-green light in the concavity centres whereas light reflected from the edges is blue [8] (d, left). By observing the scales in a light microscope with crossed polarisers, light reflected from the concavity centres is extinguished (d, right), while blue light from four segments of the concavity edges is detected, because of a polarisation rotation caused by its double reflection inside the concavity. This polarisation rotation results partly from the out-of-plane reflections at each interface geometrically rotating polarised light by an angle of $2 \psi[10](\mathbf{f})$. Spectral maps of some of the concavities confirm the optical anisotropy of the scale surface for unpolarised light ( $g$, left). Corresponding spectra (g, right), taken along the white arrow, displayed in the spectral map, show the shift in reflectance peak from green at the very edge of the concavity to blue close to the perimeter and back to green at the centre of the concavity. The red curve represents the predicted reflectance of the multilayer for normal light incidence. 
multilayer [7] (Fig. 6.1d, e).

For normal light incidence, the cuticle-air multilayer shows a reflectance peak at a wavelength of $\lambda_{\max }=525 \mathrm{~nm}$, which shifts to $\lambda_{\max }=477 \mathrm{~nm}$ for light incident at an angle of $45^{\circ}$. Light from the centre of the cavity is directly reflected, while retroreflection of light incident onto the concavity edges occurs by double reflection off the cavity multilayer (Fig. 6.1g). This double reflection induces a geometrical polarisation rotation [10]. If light, that is polarised at an angle $\psi$ to the initial plane of incidence, is retro-reflected by the double bounce, it will pick up a polarisation rotation of $2 \psi$ and the intensity distribution through collinear polarisers is therefore given by $\cos ^{2}(2 \psi)$. This leads to an interesting phenomenon: When placing the sample between crossed polarisers, light reflected off the centres of the cavities is suppressed, whereas retroreflected light from four segments of the cavity edges is detected [10, 11] (Fig.6.1d, right).

In microstructures without this double reflection, both the colour mixing and polarisation conversion are absent. This is the case for scales of Papilio ulysses, a relative of P. blumei and P. palinurus, which has considerably shallower concavities [8] that cannot retroreflect incident light, and thus shows only a more conventional shimmering blue/violet colour.

\subsection{The replication procedure}

The replication of natural photonic structures is useful for the creation of model systems to better understand structural colour in nature. Here, we demonstrate the replication of the periodically shaped multilayer structure of the Papilio butterfly scale in only five steps (Fig. 6.2). Avoiding the full structural complexity based on alternating solid cuticle and cuticle-pillar-supported air layers, we aim primarily to reproduce its optical characteristics. The multilayer structure of the artificial mimic is instead realised with two solid inorganic constituents. This results in a much simpler structural design but with the appropriate materials, structurally simplified replicas with authentic optical 


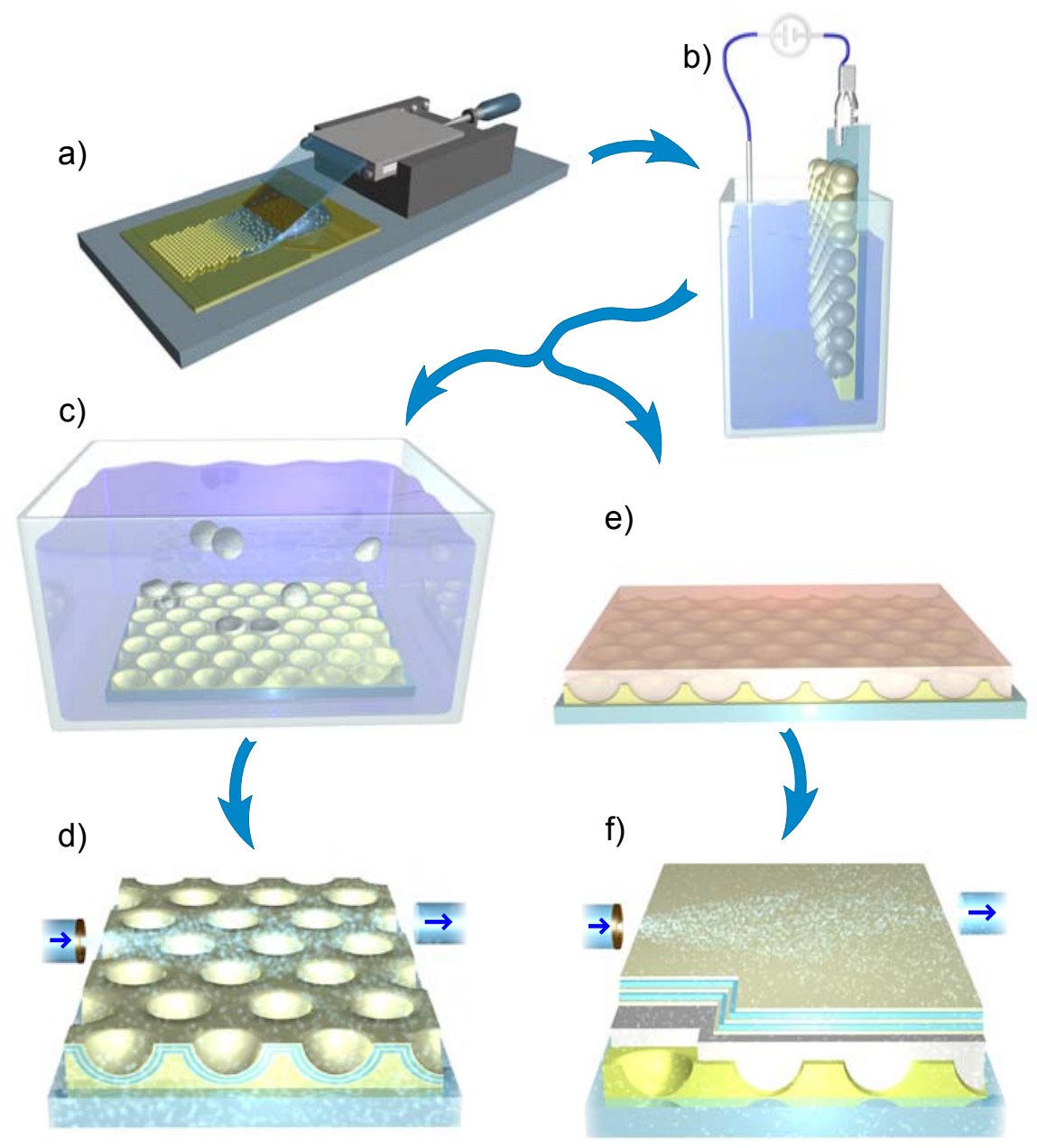

Figure 6.2: Sample fabrication. a) Deposition of polystyrene colloids on a gold-coated silicon substrate. b) Growth of platinum or gold in the interstices of the colloidal array via electro-plating. The metal deposition is terminated when the thickness of the deposited film equals the microsphere radius. c) Removal of the polystyrene spheres from the substrate by ultra-sonication in acetone. d) Sputtering of a thin carbon film and atomic layer deposition of a stack of 11 alternating $\mathrm{TiO}_{2}$ and $\mathrm{Al}_{2} \mathrm{O}_{3}$ layers (arrows indicate the precursor gas flow). e) In a second route, the colloids are molten to cover the cavities with a homogeneous film (e), onto which the $\mathrm{TiO}_{2}$ and $\mathrm{Al}_{2} \mathrm{O}_{3}$ multilayer is deposited (f).

performance are realised.

In order to create regularly arranged concavities of appropriate dimensions, polystyrene colloids of $5 \mu \mathrm{m}$ diameter are assembled on a gold-coated silicon substrate. Subsequently, a $2.5 \mathrm{\mu m}$ thick layer of platinum or gold is electrochemically grown into the interstitial space between the colloids, creating a negative replica [12-14]. Ultrasonication of the sample in dimethylformamide or acetone removes the colloids, result- 

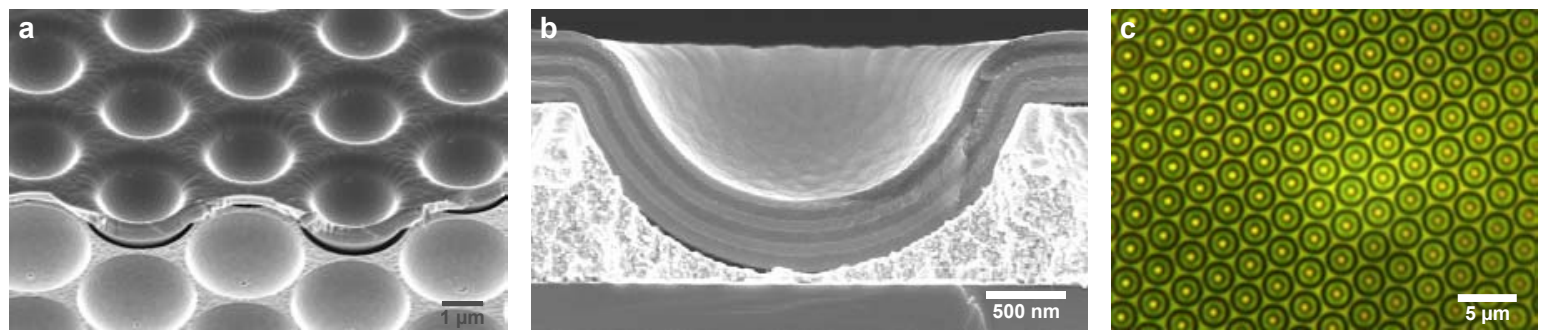

Figure 6.3: A prototype. a) Scanning electron micrograph showing a multilayer coating on top of the platinum concavities. b) A cross-section of one concavity, obtained by cryo-fracture. c) The multilayer concavities observed in the optical microscope.

ing in a template of hexagonally arranged metal concavities. A $\sim 20 \mathrm{~nm}$ thick carbon film is sputtered onto the gold surface. Finally, a conformal multilayer of thin quarterwave titania and alumina films is grown by atomic layer deposition [15]. The carbon layer between the gold (or platinum) and the multilayer stack adsorbs light that passes through the multilayer stack, reducing specular reflections and unwanted destructive interferences which otherwise severely limit the optical performance. A prototype is shown in Figure 6.3.

Optical analysis of the natural butterfly structure and the artificial mimic is performed using a micro-spectroscopic setup (see Section 3.4.2) that allows the collection of spectral data in a sample region of less than $1 \mu \mathrm{m}$ in diameter. The two-dimensional translation of the sample under the objective of the microscope enables the acquisition of spectral maps across several concavities.

\subsection{A structural replica}

The artificial mimic presented in this work is composed of multilayer concavities of $\sim 4.5 \mu \mathrm{m}$ in diameter and $\sim 2.3 \mu \mathrm{m}$ in height (Fig. 6.4a, b). The multilayer consists of 11 alternating $(57 \pm 4) \mathrm{nm}$ thick titania and $(82 \pm 4) \mathrm{nm}$ thick alumina layers. These particular layer thicknesses were chosen in the attempt to create a quarter wave stack with stop-band centre wavelength in the green - yellow spectral range (around $550 \mathrm{~nm}$ ) to match the reflectance band of the natural Papilio-structure closely. The refractive 

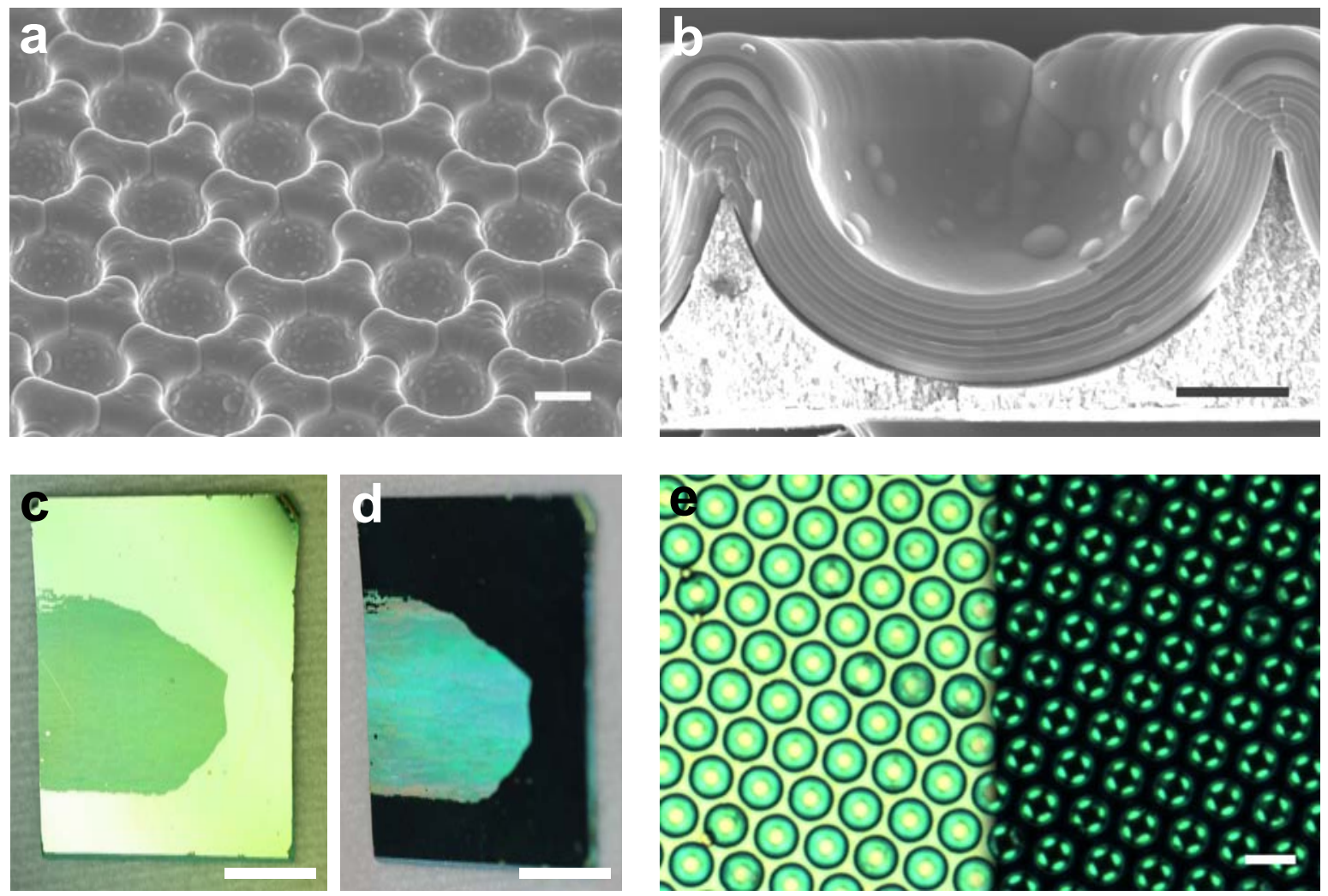

Figure 6.4: An artificial optical mimic. SEM images show the concavities which are covered by a conformal multilayer stack of 11 alternating layers of titania and alumina: a) top view, $2 \mu \mathrm{m}, \mathrm{b})$ cross-section, $1 \mu \mathrm{m}$. At perpendicular light incidence the artificial replica appears green (c), while it reflects blue at grazing incidence (d), showing some iridescence, scale bars $5 \mathrm{~mm}$. e) Under a light microscope, the concavity edges appear turquoise, while the centres and interstitial regions are yellow, scale bar $5 \mu \mathrm{m}$.

indices of the titania $\left(\mathrm{n}_{\mathrm{TiO}_{2}}=2.5 \pm 0.1\right)$ and alumina $\left(\mathrm{n}_{\mathrm{Al}_{2} \mathrm{O}_{3}}=1.7 \pm 0.1\right)$ layers were measured by ellipsometry. Both materials show no significant optical adsorption in thin films. The peak reflectance wavelength of the multilayer at $\sim 550 \mathrm{~nm}$ normal light incidence (i.e. in the centres of the cavity) corresponds well to theoretical predictions. Eleven layers are sufficient to achieve a peak reflectivity of more than $95 \%$. These multilayer coated structures immediately display iridescent colours (Fig. 6.4c, d).

The bandwidth of the butterfly scale reflectance peak $\Delta \lambda \approx 105 \mathrm{~nm}$ is slightly larger, than expected for a flat multilayer $\Delta \lambda_{\mathrm{th}}=\frac{2}{\pi} \bar{\lambda} \frac{\Delta \mathrm{n}}{\overline{\mathrm{n}}} \approx 74 \mathrm{~nm}$ where $\bar{\lambda}$ is the peak-centre wavelength, $\Delta \mathrm{n}$ the refractive index difference, and $\overline{\mathrm{n}}$ the average refractive index of the multilayer materials [16]. For the original butterfly structure, the refractive index of the 

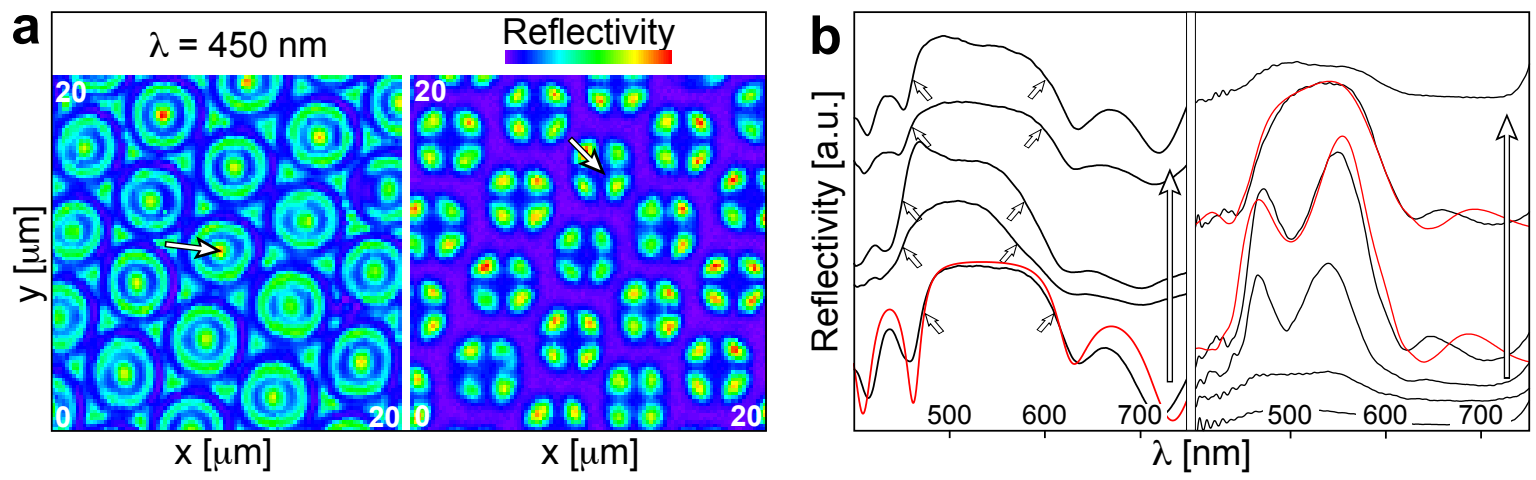

Figure 6.5: Spectroscopic analysis of the artificial mimic. a) The anisotropic reflectivity of the concavities is clearly visible in spectral maps, shown for $\lambda=450 \mathrm{~nm}$ for unpolarised light (left) and between crossed polarisers (right). b) Reflectivity along the paths indicated by arrows in the two frames in (a), respectively. Left: The reflectance peak-shift across the concavities is clearly visible. The small arrows indicate the reflectance band edges. The red curve represents the predicted reflectance of the multilayer for normal light incidence. Right: Light transmitted through crossed polarisers has undergone a polarisation rotation via a double- or triple-bounce. The double-bounce results in a single reflection peak and a triple-bounce induces a double-peak feature. The red curves model the reflection as a superposition of double- and triple-bounce.

solid cuticle layers is taken as $\mathrm{n}_{\text {cuticle }}=1.56$ [11]. For the cuticle-pillar-supported air layers a volume-fraction-averaged refractive index of $\sim 1.25$ was determined, which corresponds well to values reported in the literature [9]. Using this combination of refractive indices in the modelling of the reflection gave good fits to the data (Fig. $6.1 \mathrm{~g}$, right). In comparison, the reflectance peak width of the artificial structure $\Delta \lambda \approx 140 \mathrm{~nm}$ $\left(\Delta \lambda_{\mathrm{th}}=134 \mathrm{~nm}\right)$ is broader because of the higher ratio $\frac{\Delta \mathrm{n}}{\overline{\mathrm{n}}}$ of $\mathrm{TiO}_{2}$ and $\mathrm{Al}_{2} \mathrm{O}_{3}$.

Optical microscopy images show a similar colour variation from the concavity centres to their edges for both the natural structure and the replica (comparison of Fig. 6.4 e and Fig. $6.1 \mathrm{~d}$ ). This is confirmed by the peak shift in the corresponding spectra (Fig. $6.5 \mathrm{~b}$, left). The experimental data is well described by results of theoretical modelling of the multilayer structure. The anisotropic reflectance of the sample is clearly visible in the spectral maps (Fig. 6.5a, left).

The observation of the artificial mimic between crossed polarisers leads to a similar effect described above for the butterfly structure. Only light incident onto four segments of the concavity edges is detected. The local surface normal of $\sim 45^{\circ}$ gives rise to a 
double reflection at opposing cavity walls causing a polarisation rotation (Fig. 6.5b, right). As expected, this light is blue-shifted with respect to the light reflected from the centres of the concavities. The artificial mimic therefore displays the same optical characteristics as the natural Papilio blumei wing scale structure.

In addition, a new feature arises from the double reflection of a concave high refractive index contrast dielectric stack, which is the two-peak reflectivity seen in Figure $6.5 \mathrm{~b}$ when the sample is placed between crossed polarisers. The origin of this spectrum goes beyond the purely geometrical polarisation conversion, but depends on the different complex reflection coefficients, $r_{\mathrm{s}}, r_{\mathrm{p}}$, for $\mathrm{s}$ - and p-polarised light reflected from the multilayer. Both the magnitude and phase of $r_{\mathrm{s}}$ and $r_{\mathrm{p}}$ differ at different wavelengths relative to the reflection stop-band, with a spectrally-narrower stop band for p-polarised light.

Assuming that most of the light is retro-reflected by a single- (1), double- (2), or triple-bounce (3) (Fig. 6.6 a), a full analytical model of the concavity reflection behaviour was devised using a modified transfer matrix approach which takes into account the different origins of polarisation conversion. For all three considered light paths, the reflection spectra were calculated for linearly polarised incident light and polarisation-insensitive detection (D), with the analysing polariser aligned collinear to the input polariser ((D), and with orthogonally oriented input and analysing polarisers $(\bigoplus)$ (Fig.6.6 c-e). As expected, the reflection spectra obtained without analysing polariser equals the sum of the reflection spectra acquired with collinear and with crossed polarisers for all light paths (Fig. $6.6 \mathrm{~b}$ ).

The results show that the double bounce alone cannot produce a double-peak feature. However, retro-reflection via a triple-bounce also occurs [10] when light hits the outer edge of a concavity at an angle of $\sim 60^{\circ}$. For this triple bounce, the combination of geometrical polarisation, relative-phase-shift-induced ellipticity, and polarisation conversion based on a reflectivity difference leads to the observed double-peak structure. The reflection data is well fit by superposition of reflections from both double- and triple-bounces (red curves in Fig. 6.5b, right). Although the triple-bounce reflection 

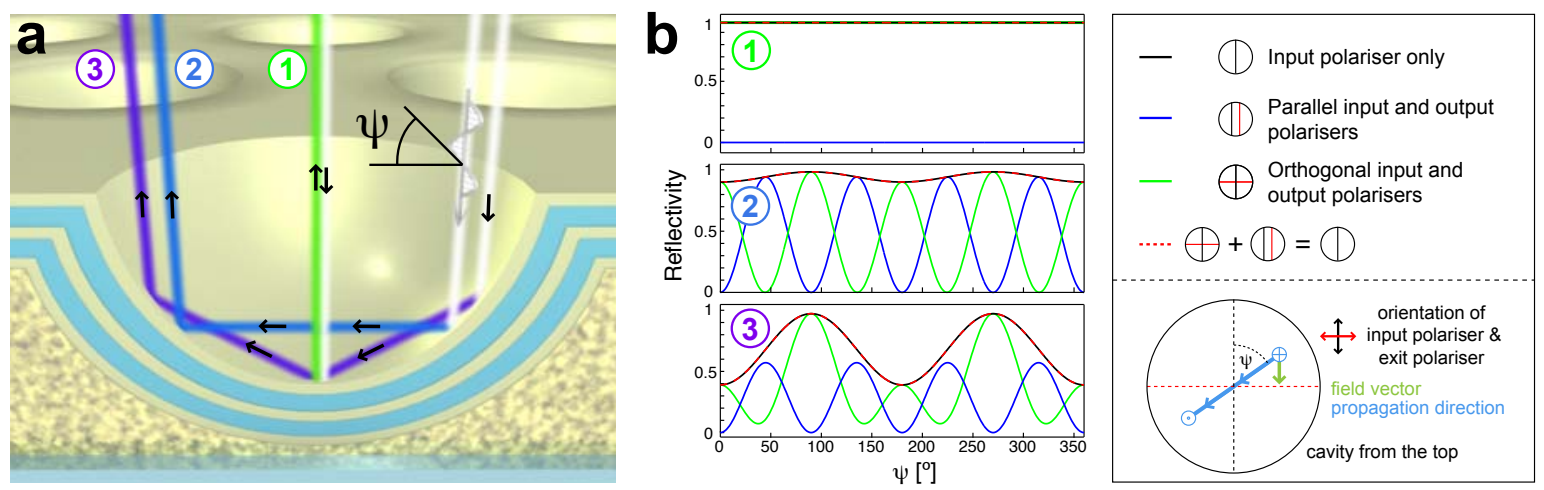

C Retro-reflection by a single bounce
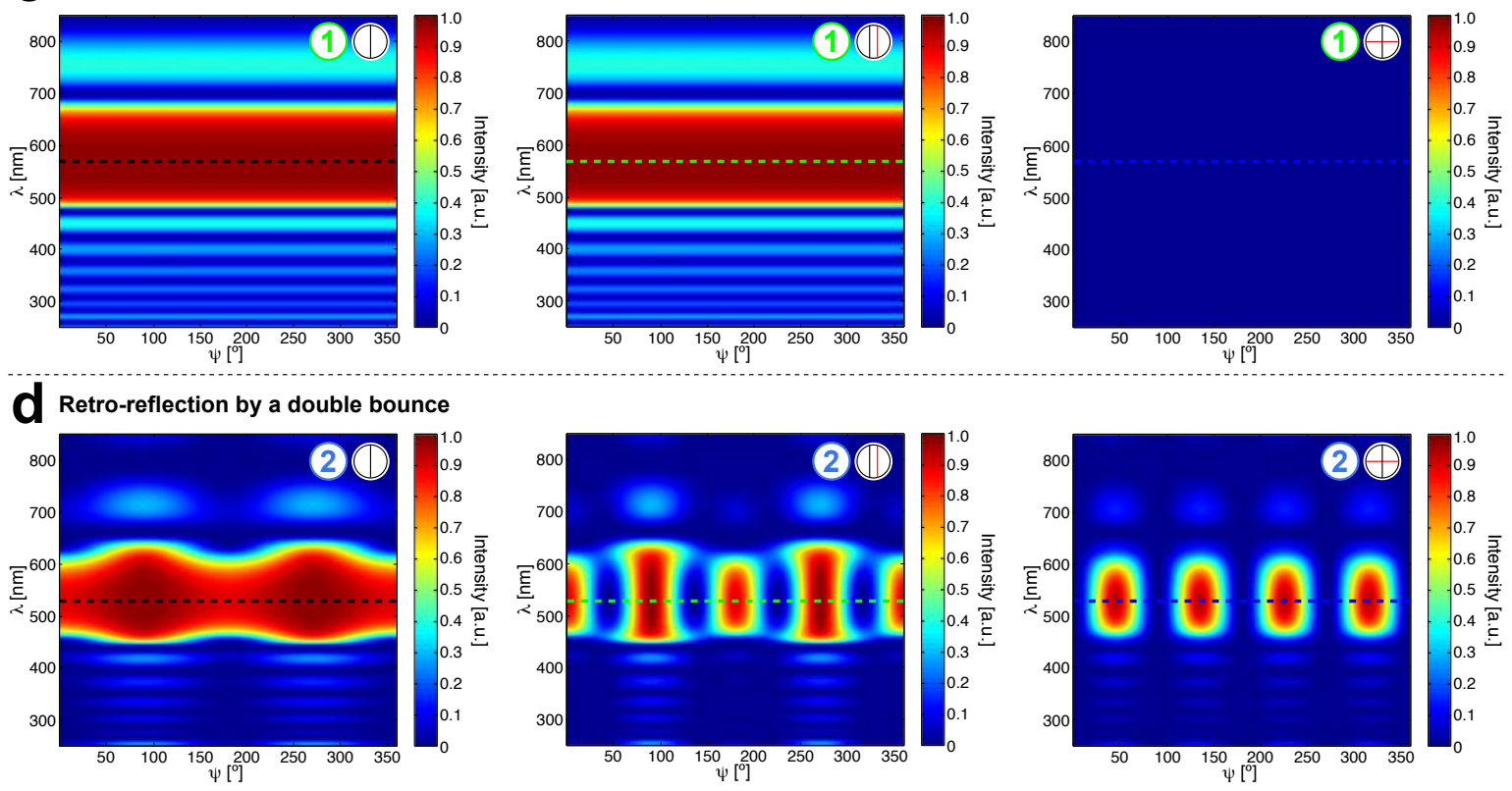

Q Retro-reflection by a triple bounce
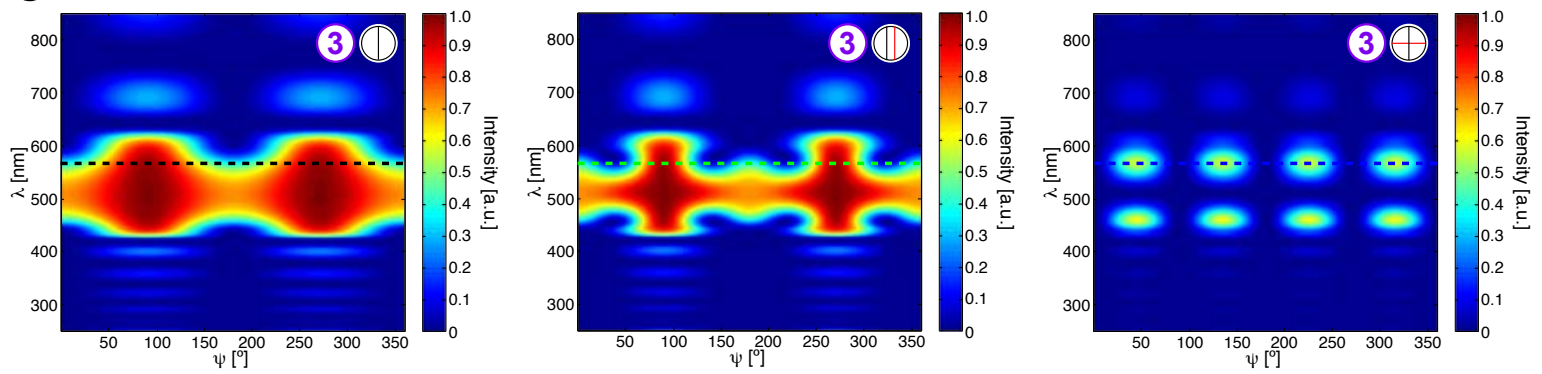

Figure 6.6: Model of the cavity retro-reflection. a) Light is retro-reflected off the concavity surfaces by a single- (1), double- (2), or triple-bounce (3). b) For the different polariser configurations, the intensity of the reflected light of given wavelength $\lambda$ depends on the polarisation $\psi$ of the incident light. For single- (c), double- (d), and triple-bounce $(\mathbf{e})$, the reflection spectra obtained without analysing polariser (D) is equal to the sum of the reflection spectra acquired with collinear (DD) and with crossed polarisers $(\bigoplus)$ The black, green, and blue dashed lines mark the wavelength for which the reflectivity is shown as a function of $\psi$ in (b). 
can also take place in the natural Papilio scale concavities, the double-peak feature is not experimentally discernible for two reasons: (1) Optical modelling shows only a shallow dip in the triple-bounce reflection peak for the butterfly's air-cuticle multilayer concavities. (2) Naturally occurring variations in concavity size and orientation lead to a mixing of the triple- and double- bounce signal, obscuring the faint double-peak feature entirely.

The angular change in peak reflectance wavelength $\lambda$ follows the simple relation

$$
m \frac{\lambda}{2}=d_{1} \sqrt{n_{1}^{2}-\sin ^{2} \theta_{0}}+d_{2} \sqrt{n_{2}^{2}-\sin ^{2} \theta_{0}}
$$

where $n_{1}, n_{2}, d_{1}$, and $d_{2}$ denote the refractive indices and thicknesses of the two different multilayer materials, $\theta_{0}$ is the light incidence angle and $m$ is a positive integer. Using titania-alumina multilayers, peak wavelength shifts from the $45^{\circ}$ double reflection of $35 \mathrm{~nm}$ can be achieved, compared to $60 \mathrm{~nm}$ for the natural butterfly structure. The use of a material with a smaller refractive index $n<n_{\mathrm{Al}_{2} \mathrm{O}_{3}}$ for the low refractive index component in the multilayer stack will increase this peak wavelength shift.

Alternatively, using a combination of high refractive index dielectrics with lower refractive index contrast (for instance zinc oxide and titanium oxide, $\frac{\Delta n}{\bar{n}} \approx 0.19$ as opposed to $\frac{\Delta n}{\bar{n}} \approx 0.39$ of $\mathrm{Al}_{2} \mathrm{O}_{3}$ and $\mathrm{TiO}_{2}$ ) for the multilayer coating would result in smaller reflectance band widths and consequently cause a more perceivable colour hue change across the concavities. High reflectivity with materials of low refractive index contrast involves Bragg mirrors with a larger number of layers, however.

\subsection{A structurally modified replica with enhanced optical performance}

Here, we introduce a third alternative for the enhancement of a structural colour effect. A small simplification in the manufacture strategy described above leads to a pronounced colour variation (Fig. 6.8). Starting from a self-organised colloidal mono- 


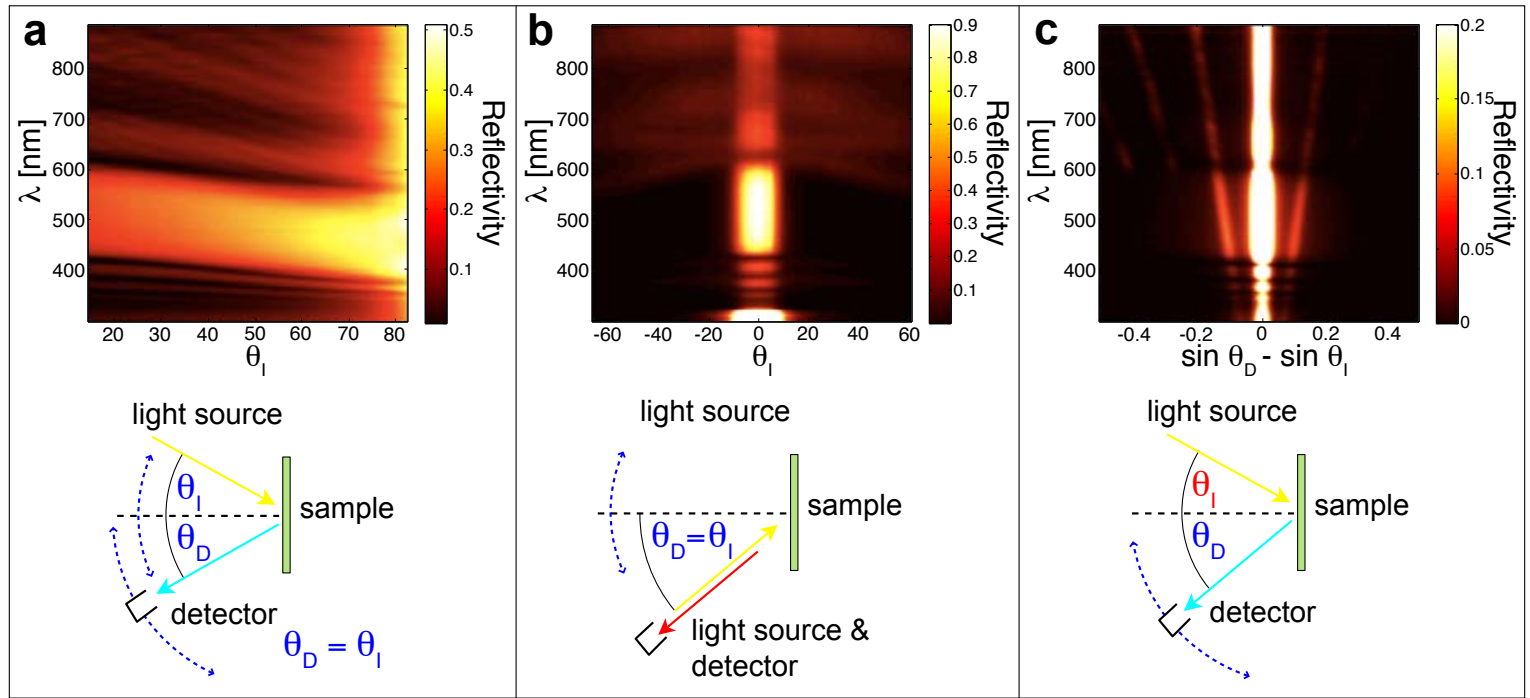

Figure 6.7: Macroscopic reflection properties of the modified replica. a) Specular reflection spectra as a function of incidence angle $\theta_{\mathrm{I}}$ show the reflection band tuning which is typical for a flat multilayer. b) The sample exhibits a remarkable amount of retro-reflection in the red independent of the light incidence angle, which is visible in the back-scattering spectra acquired as a function of incidence angle $\theta_{\mathrm{I}}$. c) Diffraction spectra as a function of detection angle $\theta_{\mathrm{D}}$ for fixed light incidence $\theta_{\mathrm{I}}$ display the typical signatures expected for the regularly arranged gold concavities. The respective measurement geometries are sketched below each set of spectra.

layer and electrochemical gold deposition, a multilayer is deposited. However instead of removing the colloids prior to ALD deposition, the sample is annealed at $200{ }^{\circ} \mathrm{C}$, the colloids melt and form a continuous film of polymer, entirely filling and covering the concavities, thereby creating a flat surface. The ALD deposition of a planar titania-alumina multilayer on top of the polymer film results in a sample with periodically shaped resonant cavities. When seen in specular reflection the surface appears bright blue. Observed in back reflection for non-normal light incidence it is strikingly red. This is confirmed in the spectra acquired in specular reflection and in backscattering from sample areas of $\sim 5 \mathrm{~mm}^{2}$ (Fig. $6.7 \mathrm{a}, \mathrm{b}$ ). Diffraction measurements show that the regularly arranged concavities cause diffraction (Fig. $6.7 \mathrm{c}$ ).

Visual information can be encoded into this photonic structure by photolithographically creating an arbitrary pattern in a $\sim 200 \mathrm{~nm}$ thick resist layer on the conducting surface prior to deposition of the colloidal template. The thin photoresist pattern does not influence the assembly of the colloids but prevents gold electro-deposition. Conse- 

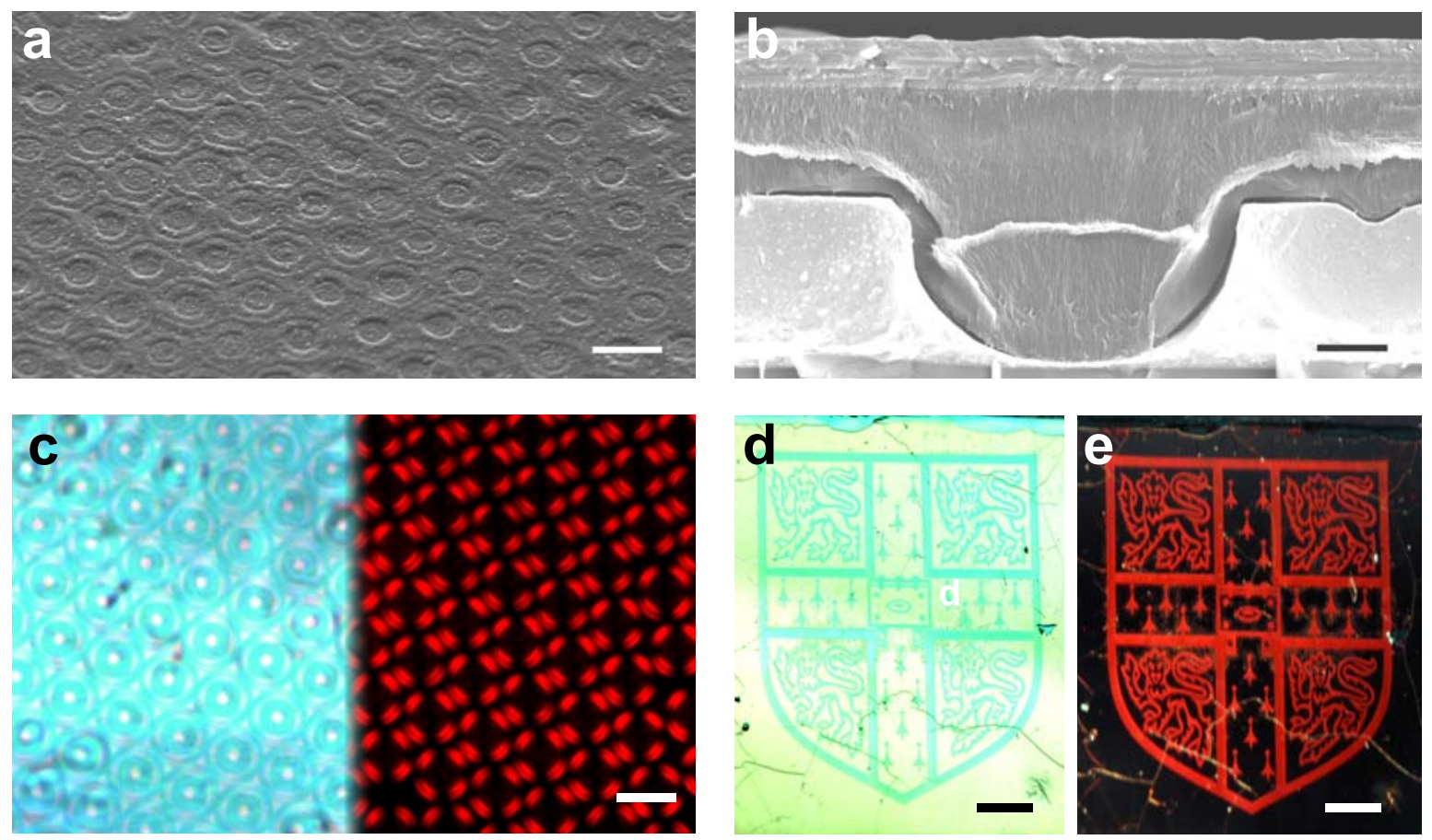

Figure 6.8: Modified mimic with enhanced optical performance. SEM images show the melted colloidal spheres which are embedded in $5 \mu \mathrm{m}$ wide gold concavities and covered by a planar multilayer stack of 11 alternating layers of titania and alumina, a) top view, $5 \mu \mathrm{m}, \mathbf{b})$ cross-section, $1 \mu \mathrm{m}$ ). Under unpolarised light, the edges of the concavities appear blue, while the centres and interstitial regions are reflecting in a broad spectral range (c, left). Between crossed polarisers only reflected red light is detected (c, right, scale bar $2 \mu \mathrm{m})$. Samples viewed in direct specular reflection (d) and in retro-reflection (e) show a striking change in colour from blue to red, scale bar $5 \mathrm{~mm}$.

quently, the concave micro-mirrors are formed only in resist-free areas thereby creating a picture, the colour of which varies dramatically with observation and light incidence angle.

The modified photonic structure has an optical signature which is similar to that of the structural replica in terms of optical anisotropy when scanning across the concavities and the behaviour in polarised light. The important difference to the conformal multilayer concavities is that the two predominant colours that are reflected on the micro-scale are not correlated by Equation 6.1. The underlying mechanism for colour creation is different. While most of the incident light with wavelengths in the blue part of the visible spectrum is directly reflected by the upper multilayer and therefore does not enter the polymer cavity, light in the red part of the spectrum is transmitted by the 

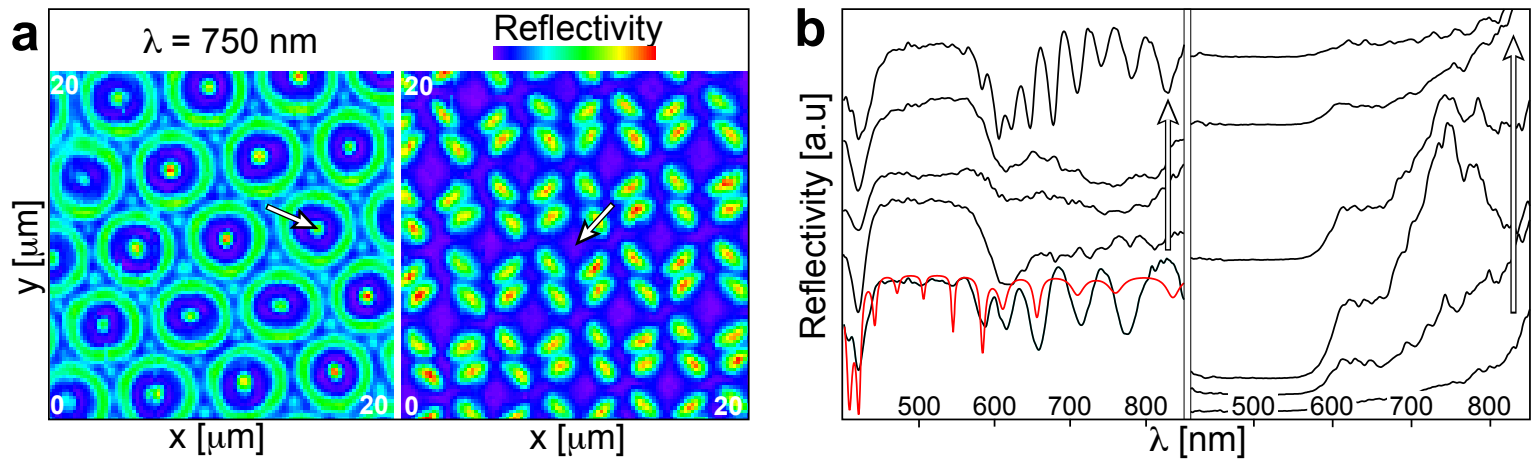

Figure 6.9: Spectral analysis of the modified copy. a) The anisotropic concavity reflectivity is clearly visible in the spectral maps for unpolarised $\lambda=750 \mathrm{~nm}$ illumination (left) and between crossed polarisers (right). Spectra from distinct points on these spectral maps along the white arrows are presented in (b). Left: change in unpolarised reflectivity varying from the concavity border to its centre. The red line shows the calculated reflectance curve of the multilayer structure for the interstitial areas. The reflectance peak resulting from the multilayer does not shift significantly across the cavity, but strong resonances in the red are observed in the concavity centres and in the interstitial areas. Right: concavity reflectivity between crossed polarisers. Only red light retro-reflected from the inclined edges accompanied by a polarisation rotation is detected.

multilayer and enters the cavities. There, most of it is reflected back in the direction of incidence by the underlying array of concave gold micro-mirrors. As expected, a blue-shift in the reflection band of the multilayer is observed for increasing incidence angles, resulting in a variation of the back-reflected colour from red to orange. Only a small fraction of red light is scattered from the inter-cavity ridges into a wide angular range.

The strong spectral selectivity of the sample is also observed in dark field microscopy, where the sample is illuminated at incidence angles $\theta_{\mathrm{I}}>70^{\circ}$ (measured from the sample normal). The numerical aperture of the used $100 \times$ objective is 0.9 which implies that it accepts light at angles $\theta_{\mathrm{D}}<65^{\circ}$. Consequently, blue light that is reflected specularly from the top Bragg mirror is not detected. Red light, that enters the cavity through the Bragg mirror, can bounce off the spherical cavity wall at angles $\theta_{\mathrm{D}}<65^{\circ}$, which results in the red appearance of the sample in dark field measurements (Fig. $6.10 \mathrm{a}$ ). Particular paths that the red light can take in the cavities are shown in Figure 6.10 b. An intensity map and spectra taken at various points in the cavities are displayed in Figure $6.10 \mathrm{c}$. 

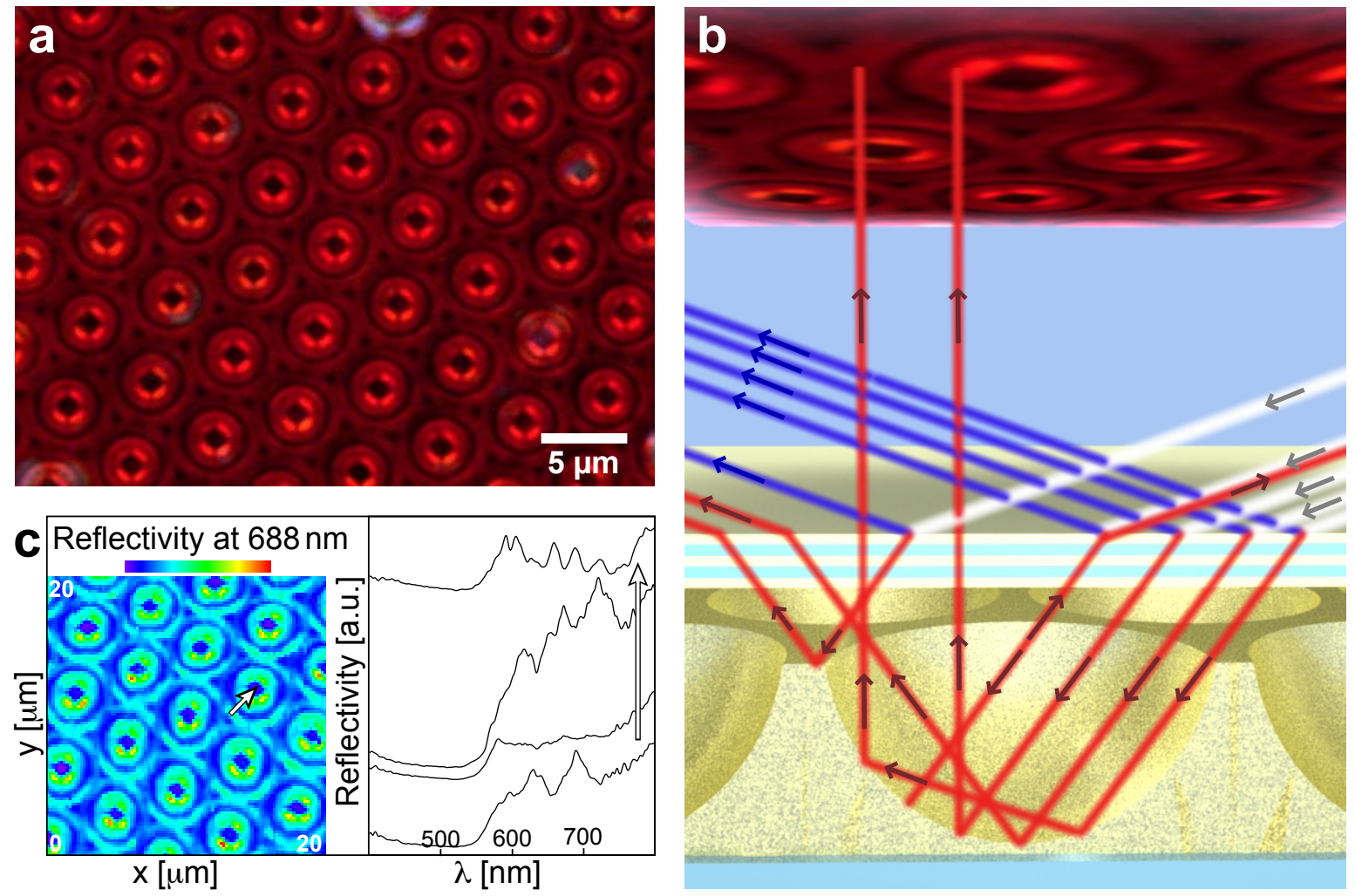

Figure 6.10: The modified mimic in the dark field. a) Dark field micrograph. b) Illustration of different light paths in the cavity. c) Intensity map of some cavities at $\lambda=688 \mathrm{~nm}$ (left) and reflection spectra (right) acquired along the white arrow shown in the intensity map.

Additional cavity resonances in the red, particularly for light reflected off the concavity centres or the interstitial regions between adjacent concavities contribute to the colour signature of the patterned device by a subtle modification in colour hue. For the thicker concavity centres, these resonances are closely spaced in wavelength, compared to the interstitial regions. The experimentally observed spectra match well with theoretical models for layer thicknesses of $\sim 3850 \mathrm{~nm}$ in the concavity centres and $\sim 1865 \mathrm{~nm}$ in the interstitial regions (Fig. 6.9b).

The sample of Figure 6.8 exhibits a pronounced variation from pale blue in specular reflection via red in all other directions to a particularly brilliant red in retro-reflection. By changing the multilayer spacing, it is possible to tune the colour seen in specular reflection across the whole visible range, accompanied by the complementary colour in retro-reflection. A change in thickness of the polymer cavity results in a variation of 
the resonant wavelengths of the light that is transmitted through the top multilayer, modifying the colour seen in back reflection for non-normal light incidence.

\subsection{Conclusion}

In summary, the intricate surface structure of Papilio butterflies was replicated in five simple steps by replicating a colloidal monolayer into an inorganic optical structure. A variation of concavity height gives rise to a colour appearance mimicking either the single coloured Papilio ulysses, (suppressing double- and triple-bounce when the concavity walls are low enough) or the colour mixing of Papilio palinurus or Papilio blumei [8]. Square-centimetre sized samples were fabricated in a facile and scaleable approach. The manufactured mimics differ in important ways from the natural counterparts: (1) by using solid inorganic materials instead of perforated cuticle lamella, the optical structure is much less fragile and a much wider range in refractive index contrast can be achieved. (2) The in-plane hexagonal symmetry of the concavities (in contrast to the quasi-1D alignment of the concavities of a Papilio wing scale) can give rise to additional grating interferences, the extent of which can be controlled by varying the conditions under which the colloidal monolayer is deposited. Furthermore, we have demonstrated that a small variation of the natural design principle allows the creation of a striking colour separation effect. Rather than the juxtaposition of two colours in Papilio butterflies, adjustable switching for any colour and its complementary hue can be achieved. Square-centimetre sized patterns and pictures with micro-scale resolution were encoded in the photonic structure, rendering this approach versatile for applications in the fields of security labelling or the manufacture of dynamic and vivid paints and coatings. This striking effect may also be used as a signalling cue in other patterned insects and should be a focus of future work. 



\section{References}

[1] Vukusic P. \& Sambles J.R. Photonic structures in biology. Nature 424, 852-855 (2003).

[2] Land M.F. The physics and biology of animal reflectors. Prog. Biophys. Mol. Biol. 24, 75-106 (1972).

[3] Doucet S. \& Meadows M. Iridescence: A functional perspective. J. R. Soc. Interface 6, S115-S132 (2009).

[4] Parker A.R. \& Townley H.E. Biomimetics of photonic nanostructures. Nature Nanotech 2, 347-353 (2007).

[5] Watanabe K., Hoshino T., Kanda K., Haruyama Y. \& Matsui S. Brilliant blue observation from a Morpho-butterfly-scale quasi-structure. Jpn. J. Appl. Phys. 44, L48-L50 (2005).

[6] Huang J., Wang, X. \& Wang Z.L. Controlled replication of butterfly wings for achieving tunable photonic properties. Nano Lett. 6, 2325-2331, (2006).

[7] Vukusic P., Sambles R.J. \& Lawrence C.R. Colour mixing in wing scales of a butterfly. Nature 404, 457 (2000).

[8] Vukusic P., Sambles R.J., Lawrence C.R. \& Wakely G. Sculpted-multilayer optical effects in two species of Papilio butterfly. Appl. Opt. 40, 1116-1125 (2001).

[9] Gaillot D.P., Deparis O., Welch V., Wagner B.K., Vigneron J.P. \& Summers C.J. Composite organic-inorganic butterfly scales: Production of photonic structures with atomic layer deposition. Phys. Rev. E 78, 031922 (2008).

[10] Coyle S., Prakash G.V., Baumberg J.J., Abdelsalam M. \& Bartlett P.N. Spherical 
micro-mirrors from templated self-assembly: Geometric reflectivity on the micron scale. Appl. Phys. Lett. 83, 767-769 (2003).

[11] Vukusic P., Sambles J.R., Lawrence C.R. \& Wootton R.J. Quantified interference and diffraction in single Morpho butterfly scales. Proc. R. Soc. London Ser. B 266, 1403-1411 (1999).

[12] Bartlett P.N., Birkin P. R. \& Ghanem M. A. Electrochemical deposition of macroporous platinum, palladium and cobalt films using polystyrene latex sphere templates. Chem. Commun., 1671-1672 (2000).

[13] Braun P. V. \& Wiltzius P. Macroporous materials - electrochemically grown photonic crystals. Curr. Opin. Colloid Interface Sci. 7, 116-123 (2002).

[14] Wijnhoven J.E.G.J., Zevenhuizen S. J. M., Hendriks M. A., Vanmaekelbergh D., Kelly J. J. \& Vos W. L. Electrochemical assembly of ordered macropores in gold. Adv. Mater. 12, 888-890 (2000).

[15] Puurunen R.L. Surface chemistry of atomic layer deposition: A case study for the trimethylaluminum/water process. J. Appl. Phys. 97, 121301 (2005).

[16] Kavokin A.V. and Baumberg J.J., Microcavities (Oxford University Press, 2007). 


\section{Conclusions and future work}

\section{Summary}

In this thesis, we studied natural layered photonic systems that induce stunning structural colours. The resulting insight, complemented by theoretical analysis of multilayer interference and diffraction of light, serves as an inspiration for the manufacture of static and tuneable Bragg mirrors, resonance cavities, patterned quasi three-dimensional photonic crystals and ultimately the replication of the intriguing wing scale structure of the butterfly Papilio blumei. The purpose of this chapter is to summarise the main conclusions of the work on natural and nature-inspired photonic systems.

In Chapter 2 some useful concepts for the theoretical calculation of multilayer reflectivity and transmission were presented. These were employed to determine the influence of design on the optical properties of multilayers, made from either polystyrene and Teflon ${ }^{\circledR} \mathrm{AF}$, polydimethylsiloxane and polystyrene-polyisoprene triblock copolymer or titania and alumina. Furthermore, the applicability of Finite Difference Time Domain simulations for the modelling of diffraction from periodic surface structures using MEEP was discussed and tested.

At the beginning of Chapter 3 some examples of natural photonic structures examined by other groups were presented. These served as models for our biomimetic efforts. In the second part of this chapter we explored the structure and optical properties of diffraction elements found on the surface of flower petals of various wild flowering plants. Structural colours have been extensively studied in the animal kingdom however, very few plants have been shown to exploit photonic structures. Our research suggests, that micro- and nano-structures, which allow specific interactions with light, have also been developed by several plant species. This implies that structural colours and photonic elements might be more wide spread in the plant world than previously thought. In a collaboration with the Plant Sciences Department of the University of 
Cambridge our results were used to show that pollinators can detect iridescence resulting from the interaction of light with periodic micro- and nano-structures. This suggests, that flowers might exploit micro-structural elements to manipulate their optical appearance to increase their attraction to pollinators, which has not yet been considered by researchers.

The manufacture of simple planar, layered optical devices based on uncommon material systems was discussed in Chapter 4. We demonstrated the manufacture of Bragg mirrors and filters from Polystyrene and Teflon ${ }^{\circledR} \mathrm{AF}$, a material combination which is promising for optical application due to the good optical contrast between the two polymers. In this context we explored the manufacture of dye-loaded resonance cavities using Teflon ${ }^{\circledR} \mathrm{AF}$-polystyrene multilayers for potential use in organic optically pumped light emitting devices. The inherent difficulties in the processing of this material system were discussed. Furthermore, an alternative material combination, consisting of polydimethylsiloxane and a common polystyrene-polyisoprene triblock copolymer, was examined. Although the refractive index contrast of this material system is rather low, the high elasticity of both components allows the manufacture of stretch-tunable layered photonic devices. We demonstrated new techniques for the assembly of multilayers from these materials and explored the optical properties of stretch-tuneable Bragg mirrors and filters. Other tuneable optical devices have been presented by various research groups however, to our knowledge this is the first realisation of planar layered interference mirrors and filters that can be tuned by stretching. These systems are beneficial for optical applications because their tuning behaviour due to uni- or biaxial strain can be easily predicted.

In Chapter 5 we investigated the potential of three different techniques, nano-imprinting, template-assisted ion milling and atomic layer deposition, for the manufacture of complex microscopic photonic structures with macroscopic areal coverage. In this context, prototypes of optical structures that cannot easily be fabricated by conventional lithography techniques were demonstrated and their optical properties studied.

Finally in Chapter 6, we discussed a successful biomimetic approach for the replication 
of the microscopic structures found on the wing scales of the butterfly Papilio blumei. This is the first time that this complex natural photonic structure has been artificially mimicked with high structural fidelity on an area of several $\mathrm{cm}^{2}$. Our detailed study of the optical properties of these artificial replicas has enhanced the understanding of light propagation within these structures. We also demonstrated that by altering the original natural blue-print different, more pronounced colour dynamics can be achieved. A slight structural modification allows any colour and its complementary hue to be separated and reflected into different directions. All the procedures involved in the manufacture, including colloidal assembly, electro-depositon and atomic layer deposition, are up-scaleable. Consequently, these biomimetic photonic structures have potential in industrial applications such as security printing, encoding of information, non-emissive display technology and other fields where distinct colours play a role. 


\section{Future work}

A prototype material system and a first set of production procedures for the manufacture of elastic multilayered optical devices has been established in the framework of this thesis. Other material systems involving fluorinated elastomers promise an increased refractive index contrast, resulting in a higher reflectivity for a set number of layers. Similarly, the inclusion of high or low refractive index nano-particles of zirconia, calcium fluoride or magnesium fluoride could enable the fine-tuning of the refractive index contrast of the layers. This way, larger refractive index contrasts than realisable with the pure elastomers could be achieved, while conserving the overall elastic behaviour of the optical device. However, the influence of the nano-particle fillers on the elastic properties of the rubber matrix needs to be investigated and alternative production routes might be necessary.

The elastic multilayers are potential systems for the creation of arrays of individually addressed pressure-tuneable optical micro-cavities (Fig.6.11). Furthermore, experiments devoted to the manufacture of strain- or pressure-tuneable optically and electri-

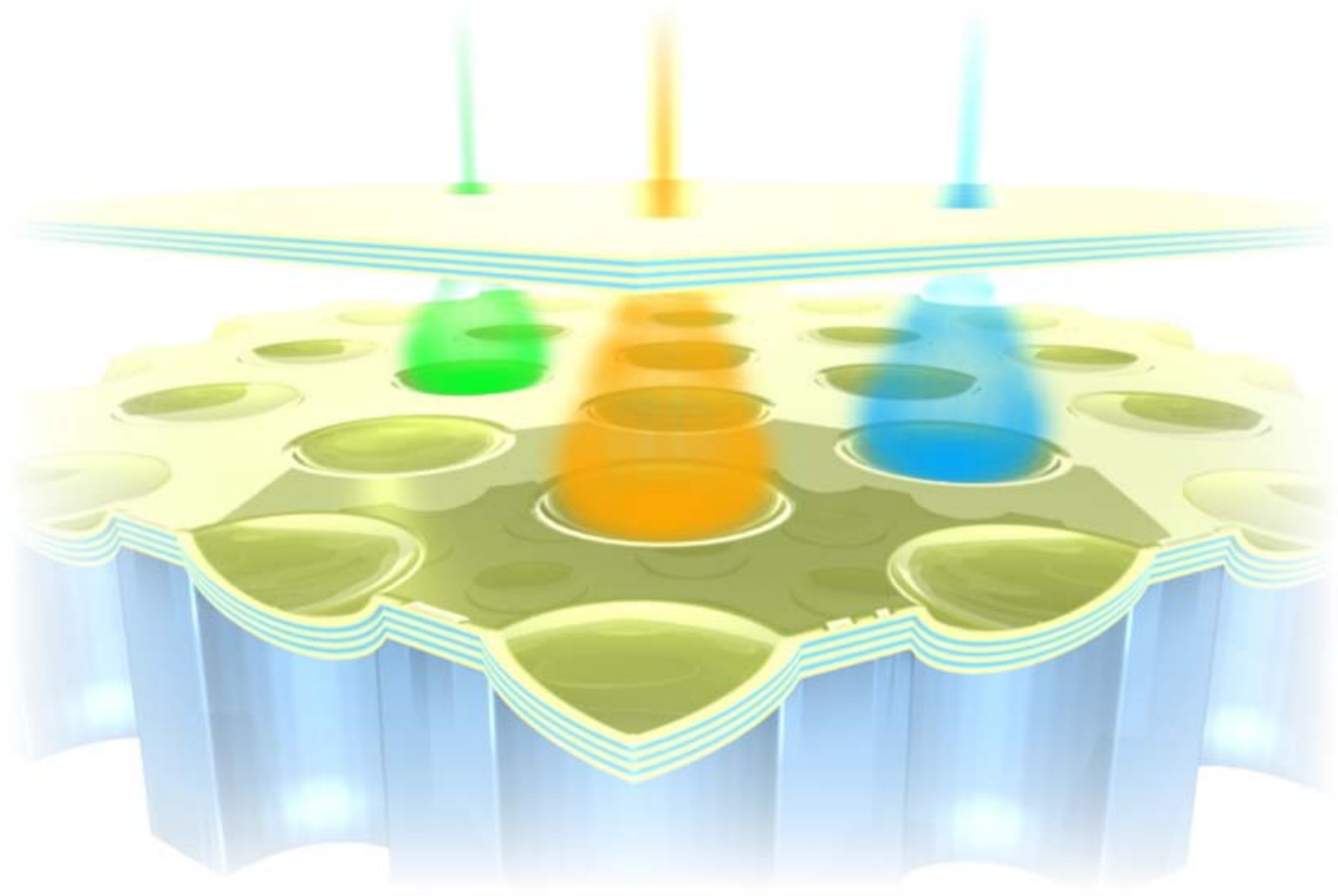

Figure 6.11: Schematic of individually tuneable elastic microcavities. 
cally pumped light emitting devices are currently conducted by including dye materials in a rubber resonance cavity between elastic Bragg mirrors.

The strong directional colour variation of the articial Papilio blumei structures combined with the significant difference in appearance when examined in unpolarised light or between crossed polarisers makes these systems promising candidates for applications in security printing. Currently the manufacture of compression-tuneable variants of the Papilio structure by using elastic materials for the top Bragg mirror is under investigation. Potentially, these structures can also be applied for the fabrication of hybrid DBR-DFB micro-lasers, combining the advantages of distributed Bragg reflector (DBR) and Distributed Feedback (DFB) lasers. To realise these systems, the inclusion of active materials that can be excited in the confinement of the Papilio-concavities has to be studied. 



\section{Acknowledgements}

Drawing this work to its conclusion with so much fun and enthusiasm was only possible due to the support and friendship offered to me by a number of people.

First, I want to thank my supervisor Prof. Ullrich Steiner. With his profound knowledge, his enthusiasm and playful wits, Ulli fostered my passion for science. When I needed space and time to spin around ideas however callow and unformed, he left me the freedom to do so, offering support, advice and confidence together with a constant stream of new ideas when necessary. When results took time to unravel, he encouraged me with his ever positive attitude and for that I am very grateful. Thank you Ulli.

I am indebted to Prof. Jeremy Baumberg, who has been a constant source of inspiration and has considerably contributed to my understanding of complex optical phenomena and to the success of my work. On top of that, he gave me the key to his optics lab, this marvellous world of toys for grown-up boys. Thank you Jeremy.

The work presented in this thesis results, to a large extent, from collaborations with excellent researchers, to whom I want to express my gratitude and some of whom I am glad to be able to call good friends. In particular these are:

Maik Scherer and Pedro Cunha, who were my cronies in pilfering natures secret optical butterfly structures; a thoroughly enjoyable endeavour.

Dr. Heather Whitney and Dr. Beverly Glover, who opened my eyes to the wonderful world of flowers. All the credit for the excellent bee work and the biological knowledge implied in our research on floral structural colours goes entirely to them. Timo Stein, a summer student in our group in 2008, who contributed to the results presented in this thesis and Katherine Thomas for our work on structural colours and her refreshing 'Englishness'.

Moritz Kreysing, who got me interested and guided me on the modelling of light propagation in complex photonic structures. His enthusiasm, logical reasoning and vast knowledge of optics is truly inspirational, making me look forward to our next discus- 
sion of another physics problem.

Bo Zheng, a Part III student in 2009, who deserves a large credit for her invaluable contribution to the work on stretch-tuneable photonic devices presented in this thesis.

Ed Crossland, whose ingenious 'Secret Science' experiments and cheerful nature are addictive.

Sven Huettner for all his inspiration in building functional (or funny) devices.

Yung-Chin Oei, who got me fascinated with image rendering software.

Dr. Piers Andrew for our fruitful discussions on optics and nano-fabrication.

Pedro, Katherine, Maik, Alex Finnemore, Moritz and Gabriel Christmann who proofread large parts of this manuscript.

Tom Mitchell for proofreading and most of all for the unbelievable and exciting marvels of nature that he never gets tired of pointing out to me.

I am grateful to my colleagues in the Thin Films and Interfaces group and the NanoPhotonics group for a unique research atmosphere. Thanks go to the people in the Nanoscience Centre, who hosted me (and my messy desk) for over three years, and especially to Sue Gymer who is a tremendous support in all practical matters.

I owe particular thanks to:

Sven Dorosz and Sven P. Krueger for all the adventures that we experienced during our physics education and their deep friendship, never hampered by the spatial distance by which we are separated.

Mum, Dad, my brother Stefan and my family for supporting and enduring my endeavours and cavorting, for all this time.

Julia for all the good that she brings to my life and for keeping my nerdiness at a tolerable level, but foremost for her cheerfulness and (hopefully) never-ending patience. Thank you! 


\section{Related publications}

"Mimicry of Papilio blumei's colourful wing scale structure". M. Kolle,

P. M. Salgard-Cunha, M. R. J. Scherer, F. Huang, S. Mahajan, P. Vukusic, J. J. Baumberg and U. Steiner, Nature Nanotech., DOI: 10.1038/NNANO.2010.101, (2010)

"Function of blue iridescence in tropical understorey plants". K. R. Thomas, M. Kolle, H. M. Whitney, B. J. Glover and U. Steiner, J. R. Soc. Interface, DOI: $110.1098 /$ rsif.2010.0201, (2010)

"Stretch-tuneable dielectric mirrors and optical microcavities". M. Kolle, B. Zheng, N. Gibbons, J. J. Baumberg and U. Steiner, Opt. Express 18, 4356 - 4364 (2010)

"Dye-sensitised solar cell based on a 3D photonic crystal". S. Guldin, S. Hüttner, M. Kolle, M. Welland, P. Müller-Buschbaum, R. Friend, U. Steiner, N. Tetreault Nano Lett., DOI: 10.1021/n1904017t, (2010)

Response to a comment on "Floral iridescence, produced by diffractive optics, acts as a cue for animal pollinators". H. M. Whitney, M. Kolle, P. Andrew, L. Chittka, U. Steiner and B. J. Glover, Science 325, 1072 (2009)

"Scalable Cylindrical Metallodielectric Metamaterials". N. Gibbons, J. J. Baumberg, C. L. Bower, M. Kolle, U. Steiner, Adv. Mater. 21, 3933 - 3936 (2009)

"Contributions of iridescence to floral patterning". H. M. Whitney, M. Kolle, R. Alvarez-Fernandez, U. Steiner and B. J. Glover, Commun. Integr. Biol. 2, $230-232(2009)$

"Floral iridescence, produced by diffractive optics, acts as a cue for animal pollinators". H. M. Whitney*, M. Kolle*, P. Andrew, L. Chittka, U. Steiner and B. J. Glover, Science 323, 130 - 133 (2009) *These authors contributed equally to this work. 